\title{
A MOLECULAR DYNAMICS STUDY OF NON-NEWTONIAN FLOWS OF SIMPLE FLUIDS IN CONFINED AND UNCONFINED GEOMETRIES
}

\author{
Remco Hartkamp
}




\section{A MOLECULAR DYNAMICS STUDY OF NON-NEWTONIAN FLOWS OF SIMPLE FLUIDS IN CONFINED AND UNCONFINED GEOMETRIES}

Remco Hartkamp 
This dissertation has been approved by:

prof. dr. S. Luding

prof. dr. B. D. Todd

Thesis committee members:

prof. dr. F. Eising

University of Twente, chairman/secretary

prof. dr. S. Luding

University of Twente, supervisor

prof. dr. B. D. Todd

Swinburne University of Technology, supervisor

prof. dr. A. van den Berg

University of Twente

prof. dr. W. J. Briels

University of Twente

prof. dr. D. M. Heyes

Imperial College London

prof. dr. J. Westerweel

Delft University of Technology

dr. J. S. Hansen

Roskilde University

dr. T. Weinhart

University of Twente

A molecular dynamics study of non-Newtonian flows of simple fluids in confined and unconfined geometries

Remco Hartkamp

Printed by Gildeprint Drukkerijen

Thesis University of Twente, Enschede, The Netherlands

ISBN 978-90-365-3532-8

Copyright (c) 2013 by Remco Hartkamp, The Netherlands 


\title{
A MOLECULAR DYNAMICS STUDY OF NON-NEWTONIAN FLOWS OF SIMPLE FLUIDS IN CONFINED AND UNCONFINED GEOMETRIES
}

\section{DISSERTATION}

\author{
to obtain \\ the degree of doctor at the University of Twente, \\ on the authority of the rector magnificus, \\ prof. dr. H. Brinksma, \\ on account of the decision of the graduation committee, \\ to be publicly defended \\ on Wednesday the $15^{\text {th }}$ of May 2013 at 14:45
}

by

Remco Marcel Hartkamp

born on 31 July 1985

in Amsterdam, The Netherlands 


\section{Summary}

Various fluid flow phenomena originate in the dynamics of the atoms that constitute the fluid. Studying fluids as a collection of atoms is key to a better understanding of, for example, non-Newtonian fluid flow behavior. Molecular dynamics (MD) is a very suitable tool for the study of fluids on the atomic level. Many MD studies have been devoted to the behavior of homogeneous, unconfined fluids under either simple shear or extensional flows, while a combination of both flow types has not been studied extensively. Strongly confined, inhomogeneous fluids are usually studied separately from homogeneous fluid problems because of their very different behavior, due to walleffects. In this thesis, a unified approach is developed, to study and compare the stresses in different flow situations.

We use MD simulations and analysis tools for: (1) the study of various properties of a simple homogeneous bulk fluid under several planar velocity fields, (2) the calculation of stresses and viscosity using the transient-time correlation function and, (3) the study of properties of an inhomogeneous fluid confined in a nanochannel.

The data suggest that the pressure tensor for a homogeneous, simple, monoatomic fluid under any planar flow field can be expressed in a unified form as a combination of equilibrium properties and non-Newtonian phenomena, such as: strain thinning viscosity, viscoelastic lagging, pressure dilatancy and out-of-flow plane anisotropy. We found consistent trends for these non-Newtonian quantities as a function of the magnitude of the strain rate tensor and the vorticity, at different state points. Similarly, interesting trends have been found for equilibrium material properties, such as the zero-shear rate first normal stress coefficient, as a function of density.

It is often not possible to directly compare experimental data to results from steady non-equilibrium molecular dynamics (NEMD) simulations. Calculating accurate timeaveraged values from these simulations is usually only feasible at deformation rates that are much larger than those accessible in experiments. We have shown that the transient-time correlation function provides a more efficient alternative to direct time- 
averaging of NEMD data. This method has been applied to an atomic fluid under combined shear and planar elongational flow, and to molecular fluids under various types of planar flow.

Non-Newtonian stresses have been studied for a simple monoatomic fluid confined in a nanochannel, where the properties vary across the channel. The pressure tensor has been expressed in terms of objective quantities, as a function of the position across the channel due to layering of the atoms. Data for various densities, temperatures and body forces have provided insight in the dependencies of various quantities. Relating the objective quantities derived from the stress tensor to local values of other state variables has not yet been fully achieved and a purely local relation between them may not exist, leaving many open questions for future research. 


\section{Samenvatting}

Velerlei stromingsfenomenen hebben een oorsprong in de bewegingen van de atomen waaruit een vloeistof bestaat. Het bestuderen van een vloeistof als een verzameling atomen is een belangrijk component voor het verkrijgen van een beter begrip bijvoorbeeld van niet-Newtoniaans stromingsgedrag. Moleculaire dynamica (MD) is een erg geschikt hulpmiddel voor het bestuderen van vloeistoffen op een atomair niveau. Vele MD studies zijn toegewijd aan homogene onbegrensde vloeistoffen onder invloed van afschuiving of extensie stromingen. Sterk begrensde, inhomogene vloeistoffen zijn meestal afzonderlijk van homogene vloeistoffen bestudeerd vanwege hun sterk verschillende gedrag, veroorzaakt door wand-effecten. in deze scriptie, een aanpak is ontwikkeld om de spanningen in een vloeistof onder verschillende stromingen op een uniforme manier te bestuderen en te vergelijken.

We gebruiken MD simulaties en analyse hulpmiddelen voor: (1) het bestuderen van allerlei eigenschappen van simpele homogene vloeistoffen onder invloed van verschillende planaire snelheidsvelden, (2) het berekenen van spanningen en viscositeit door middel van de transient-tijd correlatie functie en, (3) het bestuderen van eigenschappen van een inhomogene vloeistof in een nanokanaal.

De data suggereert dat de spanningstensor voor een homogene, simpele, edele vloeistof onder ieder planair snelheidsveld kan uitgedrukt worden in een uniforme vorm in termen van evenwichtsgrootheden en niet-Newtoniaanse fenomenen, zoals: afnemende viscositeit onder deformatie, viscoelastische vertraging, verhoging van de druk onder deformatie en anisotropie in de richting haaks op het stromingsveld. We hebben consistente trends gevonden voor deze grootheden als functie van de van de sterkte van de schuifsnelheidstensor en de vorticiteit voor vloeistoffen met verschillende dichtheden en temperaturen. Verder hebben we interessante trends gevonden voor de materiaaleigenschappen van een vloeistof in evenwicht, bijvoorbeeld de nulafschuivingssnelheid eerste normaalspanningscoëfficiënt als een functie van de dichtheid.

Het is vaak niet mogelijk om experimentele data direct te vergelijken met resul- 
taten van stationaire moleculaire dynamica simulaties uit evenwicht. Het nauwkeurig berekenen van tijd-gemiddelde waarden met behulp van dit soort simulaties is over het algemeen alleen mogelijk als de afschuifsnelheid veel groter zijn dan wat haalbaar is in experimenten. We hebben laten zien dat de transient-tijd correlatie functie een efficiënter alternatief biedt dan het direct middelen van moleculaire dynamica simulatie data. Deze methode is toegepast op een atomaire vloeistof onder een combinatie van afschuiving en planaire extensie stroming, en op moleculaire vloeistoffen onder allerlei types of planaire stroming.

Niet-Newtoniaanse spanningen zijn bestudeerd voor een simpele atomaire vloeistof in een nanokanaal, waar de vloeistofeigenschappen variëren met de positie in het kanaal. De spanningstensor is uitgedrukt in termen van objectieve grootheden, als functie van de positie in het kanaal als gevolg van laagvorming van de atomen. Data voor verschillende vloeistofdichtheden, temperaturen en drijfkrachten hebben inzicht verschaft in de samenhang tussen velerlei grootheden. Het relateren van de objectieve grootheden die afgeleid zijn van de spanningstensor aan andere grootheden is nog niet volledig bereikt en het bestaan van een volledig lokale relatie is onzeker, dit is een open probleem voor toekomstig onderzoek. 


\section{Contents}

Summary

Samenvatting iii

\begin{tabular}{lll}
\hline & Introduction & 1
\end{tabular}

1.1 Motivation . . . . . . . . . . . . . . . . . . . . 1

1.2 Historical notes and literature . . . . . . . . . . . . . . . . . 3

1.3 Hydrodynamics and transport coefficients . . . . . . . . . . . . . 5

1.4 The study of fluids with MD $\ldots \ldots \ldots \ldots$. . . . . . . . . . 7

1.5 MD simulations of very simple systems . . . . . . . . . . . . . . 10

1.6 Outline of the thesis . . . . . . . . . . . . . . . . 11

2 Molecular Dynamics Simulations $\quad 15$

$2.1 \quad$ Integration schemes $\ldots \ldots \ldots \ldots \ldots$

2.1 .1 (Velocity) Verlet . . . . . . . . . . . . . . . . . . 19

2.1 .2 Runge-Kutta . . . . . . . . . . . . . . . . . . . 19

2.2 Non-bonded interactions between atoms . . . . . . . . . . . . . . . 20

2.3 Lennard-Jones Units . . . . . . . . . . . . . . . . . . . . . . . . . . 25

2.4 Pressure and stress tensors . . . . . . . . . . . . . . . . . . 25

2.5 Thermostatting . . . . . . . . . . . . . . . . . . 31

2.5 .1 Gaussian thermostat . . . . . . . . . . . . . . . . 34

2.5 .2 Nosé-Hoover thermostat . . . . . . . . . . . . . . . . 36

2.5 .3 Braga-Travis configurational thermostat . . . . . . . . . . . . 38

2.6 Periodic boundary conditions . . . . . . . . . . . . . . . . . . . . 39

2.7 Non-equilibrium molecular dynamics . . . . . . . . . . . . . . . . . . 43 
\begin{tabular}{|lll}
3 & Homogeneous non-equilibrium molecular dynamics simulations & 47
\end{tabular}

3.1 Equations of motion . . . . . . . . . . . . . . 48

3.1 .1 Thermostatted SLLOD . . . . . . . . . . . . . . 51

3.1 .2 Molecular SLLOD . . . . . . . . . . . . . . . . . . . 52

3.2 Shear flow . . . . . . . . . . . . . . . . . . . 54 54

3.3 Elongational flows . . . . . . . . . . . . . . . . 56

3.3 .1 Planar Elongational Flow . . . . . . . . . . . . . . . 56

3.3 .2 Kraynik-Reinelt Periodic boundary conditions. . . . . . . . . . . 58

3.4 Combined shear and elongational flows . . . . . . . . . . . . 61

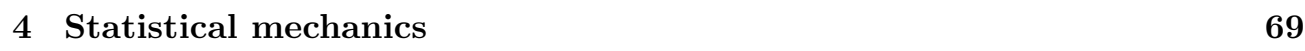

4.1 General phase space description . . . . . . . . . . . . . . . 69

4.2 Statistical ensembles $\ldots \ldots \ldots \ldots$. . . . . . . . . . . . . . . . . 72

4.3 Time-correlation functions . . . . . . . . . . . . . . . . . . 74

4.4 Calculation of Navier-Stokes transport coefficients . . . . . . . . . . 77

4.5 Transient-time correlation function . . . . . . . . . . . . . . 79

4.6 Pair distribution functions . . . . . . . . . . . . . . . . . . . 80

5 Non-Newtonian pressure tensors for simple atomic fluids 85

5.1 Viscoelasticity . . . . . . . . . . . . . . . 86

$5.1 .1 \quad$ Introduction to viscoelasticity: Cyclic deformation . . . . . . . . 87

5.1 .2 Material functions for viscoelastic fluids . . . . . . . . . . . . . . 89

5.2 Calculation of material constants from EMD simulations . . . . . . . . . 93

5.2 .1 Simulation details . . . . . . . . . . . . . . . . 94

5.2 .2 Simulation results . . . . . . . . . . . . . . . . . . . 95

5.3 Non-Newtonian constitutive models . . . . . . . . . . . . . . . . . . . . 103

5.3 .1 Rotating the pressure tensor . . . . . . . . . . . . . . . 112

5.3 .2 An objective model to describe and predict the non-Newtonian pressure tensor . . . . . . . . . . . . . . 113

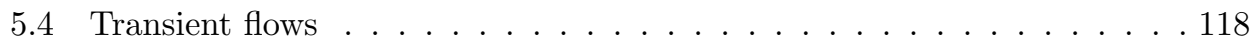

5.4 .1 Startup flow . . . . . . . . . . . . . . . . . . . . . . 119

5.4 .2 Relaxation to equilibrium . . . . . . . . . . . . . . . . 122

5.5 Summary and conclusions . . . . . . . . . . . . . 126

6 Transient-time correlation functions applied to atomic and molecular fluids

6.1 Transient-time correlation function . . . . . . . . . . . . . . . . 133

6.2 Planar Mixed Flow . . . . . . . . . . . . . . . . . . . . . . . . 135

6.2 .1 Simulation details . . . . . . . . . . . . . . 136 
6.2 .2 Atomic mixed flow results . . . . . . . . . . . . . . . . . . . . . 139

6.3 Normal stress differences . . . . . . . . . . . . . . . . . . . . . . 147

6.4 Molecular TTCF . . . . . . . . . . . . . . . . . . . . . . 150

6.4 .1 Simulation details . . . . . . . . . . . . . . . . 151

6.4 .2 Results . . . . . . . . . . . . . . . . . . . 152

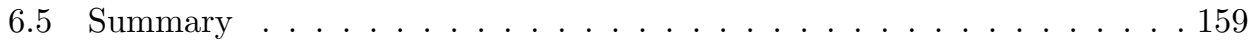

7 Confined atomic fluids 163

7.1 Literature . . . . . . . . . . . . . . . . . . . . . . 168

7.2 Simulating atomistic channels $\ldots \ldots \ldots \ldots \ldots \ldots$

7.3 Spatial averaging and macroscopic fields . . . . . . . . . . . . . 174

$7.3 .1 \quad$ Streaming velocity, strain rate and temperature . . . . . . . 178

7.3 .2 Stress calculation . . . . . . . . . . . . . . . . . . . . . 179

7.4 A study of a fluid confined in a nanochannel . . . . . . . . . . . . 181

7.4 .1 Model system . . . . . . . . . . . . . . . . . . . . . . 181

7.4 .2 Constitutive model with anisotropic stress . . . . . . . . . . . . 184

7.4 .3 Results . . . . . . . . . . . . . . . . . . . . . . 189

7.4 .4 Constitutive model . . . . . . . . . . . . . . . . . . . 206

7.5 Conclusions and summary $\ldots \ldots \ldots \ldots \ldots$

$\begin{array}{lll}8 & \text { Conclusions and recommendations } & 217\end{array}$

8.1 Homogeneous fluids . . . . . . . . . . . . . . . . . 217

8.2 Confined fluids $\ldots \ldots \ldots \ldots \ldots \ldots$

8.3 Outlook . . . . . . . . . . . . . . . . . . . . . . . 221

\begin{tabular}{|l|l|}
\hline A Derivation of the SLLOD equations of motion & 223
\end{tabular}

\begin{tabular}{|l|l|}
\hline B Validation of our objective model for the pressure tensor & 227
\end{tabular}

\begin{tabular}{ll}
\hline Bibliography & 228
\end{tabular}

\begin{tabular}{ll}
\hline Acknowledgement & 261
\end{tabular}

\begin{tabular}{ll}
\hline Curriculum vitae & 263
\end{tabular} 


\section{1}

\section{Introduction}

\subsection{Motivation}

In 1959, Richard Feynman gave a lecture that was titled: There's plenty of room at the bottom [1. This lecture is widely seen as one of the main inspirations that has led to the rapidly developing field of nanotechnology 11 The field is growing at an incredible rate and more than $30 \%$ of all scientific publications in the European Union were related to nanotechnology in 2006 [3. Besides its significant role in the scientific world, nanotechnology has also been a source of inspiration for various science-fiction writers as well as makers of games and movies [4. Many scientific and non-scientific authors have speculated in the past decades about possible applications of nanotechnology and their impact on the society and economy worldwide [5, 6]. Many of the predicted devices have not materialized yet, but the field has come a long way in the past decades with the development, fabrication and miniaturization of microdevices and nanodevices 7 11 that contain very small channels, bearings, valves or nozzles. The field is involved with mechanical, electrical, chemical and rheological processes. This thesis will be devoted to the study of fluids on a molecular level.

Our understanding of physics on the molecular level has lagged behind developments in the fabrication of new, smaller or improved devices. Theoretical, computational and experimental studies can lead to an increased understanding of phenomena and their origins. While some experiments [12 16] could predict effective global properties like relaxation time, frictional force or shear response of ultra-thin liquid films, the extraction of local values of state variables (like density, pressure and temperature) is still beyond the reach of experimental measurements. On the other hand, such local quantities can be extracted rather easily from computer simulations. Computational studies of fluids can either be done by assuming that the fluid can be approximated

\footnotetext{
${ }^{1}$ The term 'nanotechnology' was first used by Tanuguchi [2], in 1974.
} 
as a continuous medium, or from a first-principles approach, that treats the fluids as a collection of molecules. Both approaches are discussed in this chapter.

In the former approach, the system is subdivided in small fluid elements. The size of a fluid element has to be big enough so that it corresponds to a sufficiently large number of molecules, such that random fluctuations of molecules have no notable effect on instantaneous local quantities. On the other hand, solving a flow problem using very large fluid elements results in a poor spatial resolution. Many flow problems involve a flow through or around an object. When this is the case, suitable boundary conditions are needed in order to calculate the macroscopic quantities (e.g., density, pressure, velocity and temperature) in each fluid element. Many continuum models assume a no-slip boundary condition for the fluid-solid interface (i.e., the fluid velocity equals the velocity of the interface at the location of contact). Furthermore, continuum models require knowledge prior knowledge about the transport properties of the fluid in order to calculate the macroscopic quantities in each fluid element.

The use of fluid elements that are much larger than the molecular diameters is often not permitted, or even possible, in geometries that have a characteristic length in the micrometer or nanometer range $2^{2}$ Also, the no-slip boundary condition is known to be inaccurate on the molecular scale.

A first-principles approach, such as molecular dynamics (MD, see Chapter 2), does not suffer from this limitation. How much mass, momentum and energy are transported, or how large the slip length of a fluid-solid interface is, are results of MD simulations, rather than being required prior knowledge to address a flow problem. Therefore, MD is a suitable method for the study of dense confined flowing liquids.

The behavior of fluids in a nano-confined geometry often deviates from those in larger geometries. This is due to a lack of separation between the characteristic length scale of the system and the atomic length scale. For example, the balance between surface forces (such as pressure) and volume forces (such as gravity) shifts towards the surface forces as the length scale of the problem decreases. A good understanding of dynamical, structural and chemical properties of materials on a molecular level benefits the development and improvement of nanodevices and opens the door to possible new applications.

For the study of a bulk fluid, the continuum approximation may be permitted and wall slip is not considered in that case. However, a continuum treatment of the problem might still not be preferred over a first-principles simulation method. A continuum treatment with phenomenological closure relations would require knowledge from experimental studies, which is not always available and much more costly than computer simulations. Deviation from Newtonian rheology finds its origin in the mi-

\footnotetext{
${ }^{2}$ Whether this assumption holds depends not only on the size of the system, but also on the type of fluid and on its density and temperature [1].
} 
crostructure of a fluid. Since finding a molecule-based theory to describe the rheology of dense liquids is still an open problem [10], a first-principles computational study is again suitable to increase the understanding of non-Newtonian rheology of fluids that behave Newtonian under certain conditions. The literature about non-Newtonian rheology is vast and mainly concentrated on polymeric materials. Yet, even simple atomic fluids exhibit non-Newtonian phenomena under large enough deformation rates. These fluids are computationally cheaper to study and their simpler dynamics can show more clearly what mechanisms are responsible for non-Newtonian phenomena. The same mechanisms may play an important role in the rheology of polymers or other complex fluids.

MD simulations are highly computationally expensive compared to a continuum approach. This is due to the fact that the interactions of atoms with many surrounding atoms need to be calculated at every time step and that the spatial resolution in $\mathrm{MD}$ is much larger than in continuum methods, where a fluid element needs to be large compared to atoms. The computational cost poses a limitation on the number of atoms and the number of time steps in a typical MD simulation. Depending on the given fluid problem, other particle-based methods could be more suitable or computationally cheaper. Examples are: Monte Carlo (MC), direct simulation Monte Carlo (DSMC), stochastic rotational dynamics (SRD), Brownian dynamics (BD), density functional theory (DFT), smoothed-particle hydrodynamics (SPH) and dissipative particle dynamics (DPD). Many of these methods are, compared to MD, less suitable for the simulation of dense liquids and they will not be discussed in this work. For a discussion of the application of various of these methods to molecular modeling, see for example Ref. [17.

In this thesis, we use MD simulations in conjunction with statistical mechanics methods to study the structural and dynamical properties of simple atomic fluids, such as Argon, Krypton or Xenon 3 in confined and unconfined geometries. Much of the work presented in this thesis is related to calculating stresses and shear viscosity, quantifying non-Newtonian stress behavior and developing a unified approach to study the stresses in a fluid and express them in quantities that are invariant to rotation and translation of the coordinate system.

\subsection{Historical notes and literature}

Thermodynamics and statistical mechanics form the basis of many methods that are used in molecular dynamics. While thermodynamics and statistical mechanics go far

\footnotetext{
${ }^{3}$ These fluids exhibit no chemical reactions and are mono-atomic noble gases that can be approximated well by spheres.
} 
back, computer simulations came relatively recently, first in the form of equilibrium molecular dynamics (EMD) simulations. In 1959, Alder and Wainwright [18] were the first to report results from simulations that contained more than 100 interacting hardsphere particles. They showed trajectories of particles in a two-dimensional liquid and vapor in a periodic cell. The authors realized that this new simulation method offered a tool to solve many open problems in statistical mechanics. The problem they addressed in particular still raises questions nowadays: could Newton's equations of motion, which are reversible, account for the irreversible thermodynamics, such as an increasing entropy? A few years later, in 1964, Rahman 19] used a LennardJones potential to simulate a liquid consisting of 864 particles. He calculated various properties, such as the velocity autocorrelation function, the mean-square displacement and the pair distribution function. While computers have become immensely more powerful since the 60's, the methods that were used in these two studies are still commonly used in modern studies.

In 1975, a much more efficient and direct approach to calculate transport coefficients was developed by Hoover and Ashurst [20], which is called non-equilibrium molecular dynamics (NEMD). In this method, the fluid is driven away from thermodynamic equilibrium by a thermal or mechanical external field or by solid boundaries, much alike actual experiments. The same authors later used NEMD to look at hard-sphere models and Lennard-Jones fluids in order to compare their results to the Green-Kubo transport coefficients [21, 22. They found good agreement between their results and the shear viscosity of a Lennard-Jones fluid computed by Levesque et al. [23], who used EMD. Furthermore, the simulations of Hoover and Ashurst showed, for a soft-sphere fluid, the same deviation from Enskog theory that was found earlier by Alder et al. 24] for a hard-sphere fluid. The development of NEMD gave rise to a whole range of new simulations and has developed, over the last decades, into a large field of study that has provided insight in the micro-structural origins of various macroscopic phenomena.

Hoover et al. 25] showed that the DOLLS4 Hamiltonian, that couples a homogeneous driving field to the fluid, could be used to simulate adiabatic flows. The equations of motion that can be derived from this Hamiltonian are called the DOLLS equations of motion. Shortly after, Ladd [26] found that the DOLLS shear flow algorithm produced incorrect normal stress differences. Investigators, such as Ladd, Hoover, Evans and Morriss then developed the SLLOD equations of motion [26 28]. This homogeneousflow algorithm has later, in conjunction with suitable periodic boundary conditions, also been used to simulate planar elongational flow 29 31] and combined shear and elongational flow 32 .

\footnotetext{
${ }^{4}$ Named after the Kewpie doll, where 'Kewpie' is replaced by ' $q-p$ ', that represents the particles' positions and peculiar momenta, respectively.
} 
Magda et al. 33] and Bitsanis et al. 34, 36] were among the first to study strongly confined atomic liquids using MD simulations. They confined a Lennard-Jones fluid between two solid walls where the functional form of the fluid-wall interaction potential, as a function of the distance from the wall, accounted for the structure of the walls. They found a density profile that varied with the position across the channel.

The application of NEMD to confined fluids came shortly after: Liem et al. [37. mimicked a real shear-flow experiment by confining and driving the fluid via sliding atomistic walls. Harmonic springs were used to connect the particles to their respective sites in a hexagonal lattice. This approach is still used nowadays in many confinedfluid simulations. The authors compared the results from the boundary-driven shear simulations to homogeneous shear simulations in order to validate the correctness of the homogeneous shear approach. The authors argued that the homogeneous-shear algorithm removes the excess heat in an unphysical way by thermostatting the fluid everywhere in the system, whereas heat is removed via the walls in confined-fluid simulations, as is the case in experiments. It was found that the pressure tensors obtained from both approaches were in good agreement when the fluid is sheared at small shear rates. When the fluid is sheared too fast, viscous heat is generated faster than it is transported and the two approaches lead to different results.

\subsection{Hydrodynamics and transport coefficients}

Continuum methods deal with small volume elements that contain a number of atoms of the order of Avogadro's constant $N_{A}=6.02 \times 10^{23}$. Macroscopic quantities in a fluid (e.g., density, pressure, temperature) can vary with position and time, but are assumed to be homogeneous over macroscopically small fluid elements.

The governing set of equations in many continuum methods for fluids are based on the evolution of conserved quantities, for example: mass, linear momentum and energy, and their first gradients. The evolution of these quantities are respectively described by

$$
\begin{aligned}
& \frac{\partial \rho}{\partial t}=-\nabla \cdot \mathbf{J}, \\
& \frac{\partial \mathbf{J}}{\partial t}=-\nabla \cdot(\rho \mathbf{u u}+\mathbf{P})+\mathbf{F}^{E} \\
& \frac{\partial e}{\partial t}=-\nabla \cdot\left(e \mathbf{u}+\mathbf{J}_{Q}\right)-\mathbf{P}^{T}: \nabla \mathbf{u} .
\end{aligned}
$$

The mass density is denoted by $\rho, \mathbf{u}$ is the streaming velocity vector, $\mathbf{u u}$ is the dyadic product, $\mathbf{J}=\rho \mathbf{u}$ is the momentum density, $\mathbf{P}$ is the second-order pressure tensor, $\mathbf{P}^{T}$ is its transpose, $\mathbf{F}^{E}$ is an external force per unit volume, $e$ is the internal energy per unit volume and $\mathbf{J}_{Q}$ is the heat flux vector. 
This set of equations is not closed, i.e., it cannot be solved uniquely yet because the number of unknowns is larger than the number of equations. Thus, additional relations are needed to close the system. For example, a set of constitutive equations can be introduced, that relate forces and fluxes by means of transport coefficients. These coefficients can be determined either experimentally or from MD simulation data using statistical mechanics principles. In special cases, kinetic theory provides analytical predictions. Once the transport coefficients are known, a continuum fluid solver can be used to solve the closed system of governing equations.

The validity of a constitutive relation can be limited to certain conditions. For example, a relation might break down if the system is far from thermodynamic equilibrium, e.g., if the shear rate becomes too large. The critical value of the shear rate depends on the fluid and on its state point. A fluid is considered to be close to equilibrium if the following two postulates hold [28]: Firstly, the local thermodynamic equilibrium hypothesis needs to be satisfied. This hypothesis states that the principles of equilibrium thermodynamics hold for fluids close to equilibrium. In practice, this means that the gradients of macroscopic fields in the volume element are negligibly small if the driving force is small enough. The system is then said to be globally close to equilibrium and locally in equilibrium. Secondly, the entropy source strength $\sigma_{s}$ for systems close to equilibrium takes the canonical form

$$
\sigma_{s}=\sum_{i} \mathbf{J}_{i} \cdot \mathbf{X}_{i}
$$

where $\mathbf{J}_{i}$ are the phenomenological fluxes that are associated with irreversible phenomena and $\mathbf{X}_{i}$ are the conjugate thermodynamic forces. If these postulates hold, it is possible to relate the thermodynamic forces that occur in the entropy production to conjugate thermodynamic fluxes via linear transport coefficients $\mathbf{L}_{i j}$

$$
\mathbf{J}_{i}=\sum_{j} \mathbf{L}_{i j} \mathbf{X}_{j}
$$

This constitutive relation shows that when a thermodynamic force vanishes, then so will its corresponding flux and its contribution to entropy production. Substituting the appropriate constitutive relations into the governing balance equations (Eqs. (1.1), (1.2) and (1.3) reduces the number of unknowns, making it possible to solve the set of equations. This is often so complicated that solving it analytically is not feasible, such that a numerical solver is needed. The transport coefficient for a fluid is defined as a rank two tensor in general, but can be reduced to a scalar quantity in all cases considered in this study. In conventional continuum hydrodynamics, the following linear constitutive relations are often used to close the system

$$
\begin{aligned}
& \mathbf{J}_{Q}=-\lambda \nabla T \\
& \mathbf{P}=p \mathbf{I}-\eta\left(\nabla \mathbf{u}+(\nabla \mathbf{u})^{T}\right)-\left(\eta_{v}-\frac{2}{3} \eta\right)(\nabla \cdot \mathbf{u}) \mathbf{I} \text { Newton's law of viscosity }
\end{aligned}
$$


where $\lambda$ is the thermal conductivity, $T$ is the temperature, $p$ is the hydrostatic pressure and $\mathbf{I}$ the identity tensor, $\eta$ is the shear viscosity of the fluid and $\eta_{v}$ is its bulk viscosity. These closure relations are used in conjunction with assumptions, such as laminar flow and symmetry of the pressure tensor and an equation of state to calculate the hydrostatic pressure $p$ and a relation to relate the temperature to the internal energy.

\subsection{The study of fluids with MD}

The MD approach is microscopic, meaning that it calculates the trajectories of discrete objects in a many-body system. This approach is unfeasible for systems that contain a number of particles of the order of Avogadro's number, due to the enormous amount of data and computational time required. The number of particles in a MD simulation can range from hundreds to millions. A characteristic time scale in MD is given by the collision time, which is typically of the order of $10^{-15} \mathrm{~s}$. Hydrodynamic time scales, on the other hand, are coupled to the speed of sound and are typically of the order of seconds, or even larger. Dimensionless quantities, such as the Reynolds number Re $=\frac{\rho u L}{\eta}$, can be used as a characteristic number to classify flows on different scales according to the ratio of inertial and viscous forces. The Reynolds numbers in MD simulations are relatively low $(\mathcal{O}(1))$ in most cases, but turbulence can be observed in some MD simulations 38 40. As opposed to continuum solvers, MD simulations do not require specific models when turbulence is present in the fluid.

MD simulations can be used for the study of fluid problems where continuum methods are not suitable, for example strongly inhomogeneous fluids. However, the gap between the characteristic length and time scales in both methods need to be bridged in order to compare the MD results to continuum theory or couple MD results to a continuum solver, as is done in multiscale methods [41, 42].

Many flows in nature and industry can be studied using (a combination of) linear velocity profiles. Figure 1.1 shows some common (simplified) types of flow for a bulk fluid (i.e., far from any solid interface). The square represents a fluid element and the arrows outside of the cell indicate the deformations applied to the fluid. The arrows parallel to a surface represent a shear deformation, whereas arrows perpendicular to a surface represent an expansion or contraction of the fluid.

The most widely studied type of flow is (simple) shear flow. MD simulations of shear flow can either make use of homogeneous shear algorithms [43 46] or boundarydriven simulations [37, 47,49]. The former type of simulation models a bulk fluid, whereas the latter attempts to mimic a confined-fluid experiment by including walls in the simulation. Both simulation types are a subset of NEMD. What differentiates NEMD from EMD is the presence of an external force that drives the system away from 


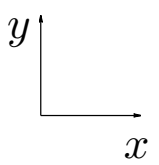

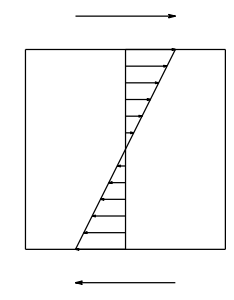

Shear

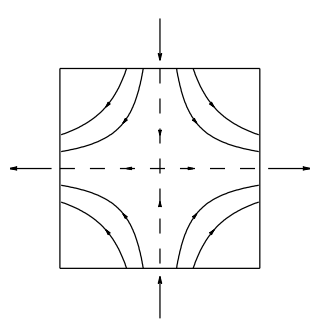

Elongation

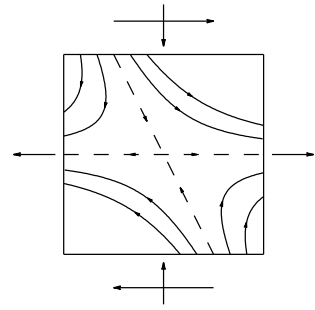

Mixed

Figure 1.1: A schematic of three planar flow types.

thermodynamic equilibrium and leads to a non-zero net transport of mass, momentum or energy. NEMD has seen a huge growth in recent years because of its potential to study transport coefficients and rheological properties of fluids in an efficient way by mimicking real experiments.

Both types of NEMD simulations are based on the same principles: Solving the governing equations of motion that describe the evolution of the phase space variables: the positions and velocities of atoms. Non-equilibrium statistical mechanics methods are used to calculate various physical quantities from the phase space variables. This is often far more difficult than in equilibrium, since some processes and definitions are less well-understood or not well-defined out of equilibrium. Temperature is an example of a quantity that is not uniquely defined out of equilibrium. For example, Hoover and Hoover [50] compared three non-equilibrium definitions of temperature.

Simulations of unconfined fluids can be used to study the properties of a bulk fluid, i.e., a fluid that is far enough removed from a confining surface, such that the surface does not affect the fluid. The boundaries of the simulation cell in such simulations are generally periodic and may not affect or disturb the flow in any way. Bulk fluids under a homogeneous flow field can be simulated by integrating a suitable set of equations of motion that couple a velocity gradient homogeneously to the atoms, i.e., the absolute positions of the atoms have no explicit relevance, only their positions relative to each other do. Since the fluids in this type of simulations are homogeneous, information can be averaged over space and the number of particles required for such simulations is typically small $\left(N=\mathcal{O}\left(10^{3}\right)\right)$.

Confined fluid simulations often aim to mimic an experiment or real-life problem in a natural way. Walls, a free surface or heat sources and sinks are explicitly modeled to replicate a real system. This type of simulation typically requires a much larger number of particle $\left(N=\mathcal{O}\left(10^{4}-10^{7}\right)\right)$ than the homogeneous approach, in which fluid properties are independent of the position. The presence of a solid surface causes the distribution of particle positions (and thus also the density of the fluid) to become a 

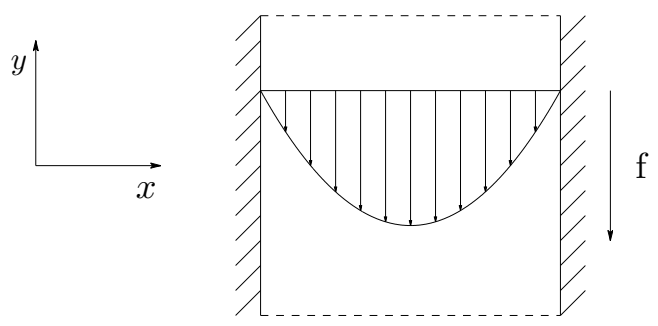

Figure 1.2: A schematic of planar Poiseuille flow.

function of the position, which affects other quantities as well. Variations in density damp out over a distance of several atomic length scales and these variations very close to an interface can often be considered negligible in 'large enough' systems. When the size of the system is within an order of magnitude from the atomic length scale, variations cannot be ignored and averaging over the volume is no longer permitted. The behavior of strongly confined, inhomogeneous fluids is far from understood. In recent years, many efforts have been made to study the transport coefficients of fluids in confined geometries and to find a constitutive relation that couples the microscopic data to macroscopic balance equations. Finding such suitable constitutive relations remains an open problem for strongly confined fluids.

Solid boundaries need to be explicitly included in MD simulations in some cases. An example is (planar) Poiseuille flow, shown in Figure 1.2. In this flow type, a fluid is confined between two parallel solid interfaces and is driven by a pressure difference or a body force, for example gravity. As the body force pushes the fluid to accelerate, viscous effects are responsible for a resistance to flow. A steady flow can form when the driving force and the viscous resistance are in balance. The resulting velocity profile is typically a quadratic function of the position across the channel.

Even simple atomic fluids are known to exhibit non-Newtonian phenomena, such as shear thinning, shear dilatancy and normal stress effects [51, 52]. Furthermore, the density in strongly confined fluids can become inhomogeneous, which affects the transport properties as well as the phase diagram (i.e., a diagram that shows in which phase or phases a material can occur at a given state point) and complicates the search for a constitutive law that describes the rheology of confined fluids. Material constants, like shear viscosity, bulk viscosity, first and second normal stress coefficients, shear relaxation modulus and bulk relaxation modulus are defined to describe Newtonian and non-Newtonian properties. With the present work we aim to make contributions to the fields of homogeneous MD simulations as well as increasing understanding of the properties of highly confined inhomogeneous fluids. 


\subsection{MD simulations of very simple systems}

Simulating large and complicated systems was not feasible in the early days of MD simulations. Computers have become more powerful in the past decades and more methods for simulating and analyzing complicated molecular systems have been developed. While very complicated systems can now be studied with MD, there still is a scientific interest in simulations of simple fluids in a periodic simulation cell or in a confined geometry. The main goal of these simulations is often not an attempt to recreate experimental systems as closely as possible, while some MD simulations of more complicated materials may have this purpose [53, 55].

Many experiments and devices that have been engineered in the past decade operate on length scales of at least several hundreds of nanometers wide, and more often in the micrometer range $5^{5}$ For the development of smaller devices one has to face challenges in the methods of fabrication, detection, flow control and surface modification [56]. Many MD simulations of simple confined fluids, on the other hand, have a characteristic length scale around $1-10 \mathrm{~nm}$. Simulations of larger systems contain many more atoms and thus become very computationally expensive. Furthermore, there is a scientific interest in understanding the dynamics and structural properties of fluids confined in nanometer geometries, since this often deviates from fluid properties in larger geometries.

The fluids that are typically used in experiments are much more complicated than the simple atomic fluids used in the MD simulations in this thesis. Although simulation methods for more complicated materials exist, there are multiple reasons to simulate simple atomic fluids. In the first place, the potential that describes the interaction between two atoms is well-understood and using this interaction potential to simulate simple fluids results in correct transport properties and phase transitions for a bulk fluid. This is often not the case for more complicated materials. Due to the simplicity of monoatomic fluids, microscopic origins of flow phenomena can be identified easier than in a fluid in which many more internal mechanisms, types of interactions and possibly chemical reactions would be active. The insights obtained from simple systems can then be used to increase the understanding of more complicated materials and geometries. Furthermore, bonds between atoms tend to vibrate at high frequencies, such that the simulation time steps need to be smaller than for monoatomic fluids. Fixing the bond lengths and angles, or even further coarse graining of the molecules, can allow for a larger simulation time step and a larger accessible number of molecules, but goes at the expense of the realism of the computer simulations. For example, many models exist that represent water molecules as a combination of atoms and electrical charges with a fixed internal structure [57]. Although these models avoid the small

\footnotetext{
${ }^{5}$ Notable exceptions are flows through or around carbon nanotubes and porous silica.
} 
simulation time steps needed to capture the fast bond vibrations, they often only partially succeed to recreate the bulk transport properties of water over a range of densities, temperatures and pressures. Furthermore, these water models are optimized to reproduce bulk properties, but tend to be less suitable to reproduce interaction between water and silica or clay.

Besides the fact that simulation systems are often much simplified in comparison to real systems, the accessible shear rates in computer simulations and experiments are often also very different [17. A method to overcome this gap is discussed in Chapter 6 .

\subsection{Outline of the thesis}

\section{Chapters 2, 3 and 4}

A variety of tools are introduced in these three chapters, that are needed for the simulations and analyses presented in later chapters. An introduction to molecular dynamics simulations is given in Chapter2. The chapter treats, amongst other things, some of the common and relevant interaction potentials, integrators and temperature control mechanisms. Next, in Chapter3, equations of motion and boundary conditions are presented that can be used to simulate homogeneous simple shear flow, planar elongational flow and combinations of shear and planar elongational flow. An introduction to statistical mechanics methods is given in Chapter 4. The methods discussed in this chapter are used for the calculation of structural and dynamical fluid properties from a sufficiently large set of simulation data.

\section{Chapter 5}

This chapter focusses on the flow behavior of simple atomic bulk fluids in equilibrium and under constant homogeneous planar flows. In particular, deviations from Newtonian behavior are studied and quantified:

- The density-dependence of the stress relaxation function of a simple fluid. The stress relaxation function as well as equilibrium material constants are calculated from the equilibrium stress autocorrelation function.

- A model is presented that predicts the pressure tensor for a non-Newtonian bulk fluid under a homogeneous flow field. The model provides a quantitative description of the strain thinning viscosity, bulk dilatancy, deviatoric viscoelastic lagging and out-of-shear-plane pressure anisotropy.

- The transient shear stress and normal stress differences in a sheared bulk fluid are studied, both for startup and for cessation of flows. Non-equilibrium molecular 
dynamics (NEMD) simulation results of the shear stress are compared with a linear viscoelastic prediction from equilibrium molecular dynamics (EMD).

\section{Chapter 6}

The transient-time correlation function (TTCF) method can be used to calculate the transient and steady-state values of various quantities for a fluid subjected to a constant deformation rate. This method is more efficient than direct averages of NEMD simulations when the deformation rate is sufficiently small. In this chapter, TTCF is used to calculate the nonlinear response of homogeneous fluids. Three flow problems are discussed:

- The TTCF response of components of the pressure tensor are studied, for a simple atomic fluid subjected to a constant planar mixed flow (PMF) of shear and elongation. The TTCF response is compared to directly averaged NEMD measurements.

- The normal stress differences in a sheared atomic fluid are calculated using TTCF. A phase space mapping is introduced in order to improve the statistics of these computationally expensive calculations.

- We study how the transients (the startup from equilibrium to non-equilibrium steady state) of the pressure tensor and the viscosity of fluids consisting of short linear chain molecules depend on the deformation rate, on the type of flow and on the length of the molecules. The modes of relaxation present in the stress autocorrelation function of a diatomic liquid are analyzed in order to increase understanding of the transient viscosity.

\section{Chapter 7}

In this chapter, the local properties of a strongly confined fluid are studied. The distribution of atoms is strongly inhomogeneous near an interface, such that the fluid density is a function of the location in the system. This, in turn, affects the local values of other state variables, and the relations between them. The chapter provides an overview of the relevant literature and required techniques for the study of an inhomogeneous fluid. In particular, we study the flow of a dense Lennard-Jones fluid confined in a rectangular channel of approximately four nanometer width. Macroscopic fields are obtained from microscopic data by temporal and spatial averaging and smoothing the data with a self-consistent coarse-graining method based on kernel interpolation. Two phenomena make the system interesting: (i) strongly confined fluids show layering, i.e., strong oscillations in density near the walls, and (ii) the stress deviates from 
the Newtonian fluid assumption, not only in the layered regime, but also much further away from the walls. Various scalar, vectorial, and tensorial fields are analyzed and related to each other in order to understand better the effects of both the inhomogeneous density and the anisotropy on the flow behavior and rheology. The eigenvalues and eigendirections of the stress tensor are used to quantify the anisotropy in stress and form the basis of a newly proposed objective, inherently anisotropic constitutive model that allows for non-collinear stress and strain-rate tensor by construction.

\section{Chapter 8}

In this final chapter, a summary is given of the work that was presented and the main conclusions that were drawn from the observations. Furthermore, we list some important questions that remain open and recommend which steps need to be taken to address these open problems. 


\section{2}

\section{Molecular Dynamics Simulations}

With the rise of micro- and nanotechnological applications, the industrial demand and scientific interest in understanding the microscopic origins of various fluid phenomena has seen a vast and rapid increase over the last decades. While experiments are valuable in gaining understanding of the rheological behavior of fluids, they are often not suitable to study what happens on a microscopic level. Simulations can lead to insights that can sometimes not be extracted from experimental measurements.

Continuum methods use macroscopic conservation equations in conjunction with constitutive relations to study the behavior of a fluid. The validity of these constitutive relations relies on the assumption that the fluid properties are approximately constant across macroscopically small volume elements. This assumption is accurate for most practical purposes, but is invalid if variations in macroscopic quantities are large over atomic time or length scales. Furthermore, constitutive relations often provide a heavily simplified model based on empirical findings that are often limited to certain conditions. Finally, to solve the closed set of governing continuum equations, transport coefficients need to be provided. In a non-Newtonian fluid, these transport coefficients are not only dependent on the thermodynamic state point of the fluid, but also on the flow field.

The limitations of a continuum approach are avoided by using a microscopic approach, such as molecular dynamics (MD). Methods based on a microscopic approach deal with discrete objects, rather than infinitesimally small volume elements. By integrating the equations of motion in time from a well-defined initial state, the method keeps track of particle positions and velocities. Macroscopic quantities, like pressure and temperature, can be calculated from this microscopic information. Furthermore, these methods give rise to dynamical, structural and chemical information that is not 


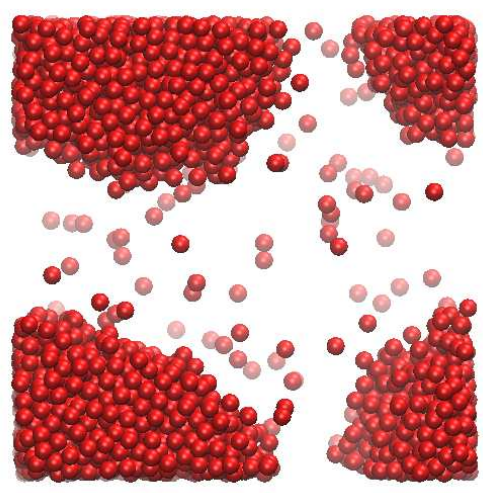

(a) Solid-Vapor mixture

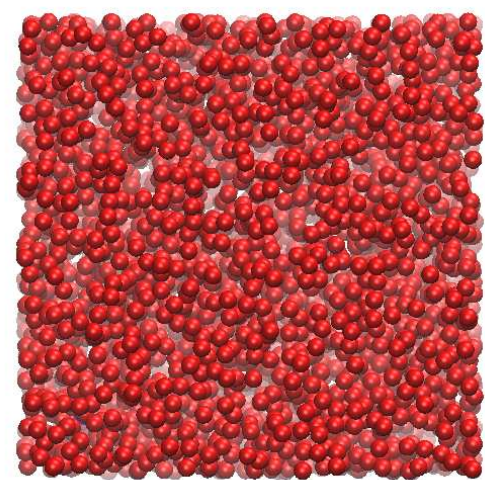

(b) Liquid

Figure 2.1: Different phases, both simulated with the same interaction potential and density, only the temperature differs.

available in continuum methods.

Different phases of a material can be created simply by changing the thermodynamic conditions, such as the temperature or density, without having to change the simulation methodology. Figure 2.1 shows two snapshots of a simulation of a dilute fluid, where the temperature is changed.

Classical Newtonian dynamics forms the basis of MD. Newton's second law of motion states that the motion of any object satisfies

$$
\begin{aligned}
\mathbf{F} & =m \mathbf{a} \\
& =m \ddot{\mathbf{r}},
\end{aligned}
$$

where $\mathbf{F}$ is the total force acting on the object, $\mathbf{a}$ is its acceleration and $m$ its mass. This equation can be written as a set of first-order differential equations

$$
\begin{aligned}
\dot{\mathbf{r}} & =\mathbf{v}, \\
\dot{\mathbf{v}} & =\frac{\mathbf{F}}{m},
\end{aligned}
$$

where $\mathbf{v}$ is the velocity of the object. If the force on the object depends only on its position, the system is said to be holonomic. Furthermore, if the forces are fully determined by the state of the system, the set of equations is called deterministic (as opposed to stochastic). This means that one can integrate the state of a system forward in time and then back to end up with the same initial state. Thus, a deterministic system of equations in conjunction with a set of initial conditions $(\mathbf{r}(0), \mathbf{v}(0))$ has a unique solution for every moment in time. 
Rather than considering a single object, MD typically deals with many-body systems, in which the dynamics of the bodies are coupled to each other via interactions. The equations of motion then become a large set of coupled first-order differential equations. The internal energy in a multi-body system is described by a Hamiltonian

$$
\mathcal{H}_{0}=\sum_{i=1}^{N} \frac{\mathbf{p}_{i} \cdot \mathbf{p}_{i}}{2 m_{i}}+U\left(\mathbf{r}_{1}, \ldots, \mathbf{r}_{N}\right)
$$

where $\mathbf{p}_{i}=m_{i} \mathbf{c}_{i}=m_{i}\left(\mathbf{v}_{i}-\mathbf{u}\left(\mathbf{r}_{i}\right)\right)$ denotes the peculiar (or 'thermal') momentum of particle $i$ relative to the streaming motion $\mathbf{u}\left(\mathbf{r}_{i}\right)$ and $U$ is the potential energy due to interactions between particles. The streaming velocity is defined as $\mathbf{u}(\mathbf{r})=$ $\sum_{i=1}^{N} m_{i} \mathbf{v}_{i} \delta\left(\mathbf{r}-\mathbf{r}_{i}\right) / \sum_{i=1}^{N} m_{i} \delta\left(\mathbf{r}-\mathbf{r}_{i}\right)$, where $\delta\left(\mathbf{r}-\mathbf{r}_{i}\right)$ is the Dirac-delta function and $\mathbf{v}_{i}$ the laboratory velocity of particle $i$. The Hamiltonian equals the total energy in the system if the fluid is in equilibrium because the streaming motion is then zero and thus the peculiar momentum equals the laboratory momentum.

For a fluid in equilibrium, equations of motion are derived from the internal energy Hamiltonian (Eq. 2.5)

$$
\begin{aligned}
\dot{\mathbf{r}}_{i} & =\frac{\partial \mathcal{H}_{0}}{\partial \mathbf{p}_{i}}=\frac{\mathbf{p}_{i}}{m_{i}} \\
\dot{\mathbf{p}}_{i} & =-\frac{\partial \mathcal{H}_{0}}{\partial \mathbf{r}_{i}}=\mathbf{F}_{i}
\end{aligned}
$$

where $\mathbf{F}_{i}$ is the resultant force on particle $i$ due to all other bodies. By default, the number of particles and the system volume are constant in time. Furthermore, these equations of motion are time-reversible and conserve energy. The latter can be shown by calculating the derivative of the Hamiltonian with respect to time

$$
\frac{d \mathcal{H}_{0}}{d t}=\sum_{i=1}^{N}\left[\frac{\partial \mathcal{H}_{0}}{\partial \mathbf{r}_{i}} \cdot \dot{\mathbf{r}}_{i}+\frac{\partial \mathcal{H}_{0}}{\partial \mathbf{p}_{i}} \cdot \dot{\mathbf{p}}_{i}\right]=\sum_{i=1}^{N}\left[\frac{\partial \mathcal{H}_{0}}{\partial \mathbf{r}_{i}} \cdot \frac{\partial \mathcal{H}_{0}}{\partial \mathbf{p}_{i}}-\frac{\partial \mathcal{H}_{0}}{\partial \mathbf{p}_{i}} \cdot \frac{\partial \mathcal{H}_{0}}{\partial \mathbf{r}_{i}}\right]=0 .
$$

This property shows that each set of motion equations that can be derived from a Hamiltonian conserves energy. Additionally, the equations of motion derived from a Hamiltonian satisfy the following identity

$$
\frac{\partial \dot{\mathbf{r}}_{i}}{\partial \mathbf{r}_{i}}+\frac{\partial \dot{\mathbf{p}}_{i}}{\partial \mathbf{p}_{i}}=\frac{\partial^{2} \mathcal{H}_{0}}{\partial \mathbf{r}_{i} \mathbf{p}_{i}}-\frac{\partial^{2} \mathcal{H}_{0}}{\partial \mathbf{p}_{i} \mathbf{r}_{i}}=0
$$

Solving the equations of motion analytically for all positions and momenta is generally not feasible. Therefore, numerical schemes are needed to integrate the equations of motion in time. In order to integrate the equations of motion, one needs to know the forces that act on each particle. The field of MD simulations has developed rapidly over the past five decades. This has accelerated the development of interaction potentials, integration schemes and thermostats. Some of the relevant techniques are 
discussed in this chapter, but for a more detailed treatment, the reader is referred to one of the many excellent textbooks related to the subject [58,61].

The outline of this chapter is as follows: The most common integration schemes are presented in Section 2.1. In Section 2.2 the most common atomic interaction potentials are discussed. The units used in MD simulations are discussed in Section 2.3. The calculation of the pressure tensor in molecular dynamics simulations is discussed in Section 2.4. Next, in Section 2.5. some of the challenges and implications of temperature control in MD simulations are discussed. Standard periodic boundary conditions for equilibrium molecular dynamics (EMD) simulations are introduced in Section 2.6. Finally, in Section 2.7 the concept of non-equilibrium molecular dynamics (NEMD) is introduced.

\subsection{Integration schemes}

In MD simulations, the governing equations are solved numerically by integrating particle positions and velocities in time. Since MD simulations typically require many time steps, it is important that an integration scheme conserves quantities, like energy and momentum. Furthermore, time-reversible integration is required for theoretical treatment of a deterministic set of equations. Finally, a large time step is preferable, without too much loss of accuracy. The error of an integration algorithm is a combination of the order $b$ of the algorithm and the step size $\Delta t$, so the global error is then $\mathcal{O}\left((\Delta t)^{b}\right)$. Since we are often interested in averages rather than individual trajectories, a large step size is often preferred over a high accuracy.

Some integrators are said to be symplectic for Hamiltonian systems. This means that they preserve the Hamiltonian, regardless of the time step. This property is practical (but not strictly required), especially given the large number of time steps in MD simulations. However, we will later see that many systems that we deal with are non-Hamiltonian (see Sections 2.5.2 and 3.1.

The appropriate simulation time step $\Delta t$ that can be used to integrate the equations of motion depends on several factors. The simulation time step has to be chosen such that the fastest microscopic processes can be calculated with a good temporal resolution. Furthermore, the time step has to be such that the integrator remains stable. We do not engage in a detailed study of the time step, as this is well-established for simple atomic fluids. Time steps of approximately $\Delta t=0.001 \tau-0.005 \tau$ are commonly used, depending on the integrator, the required accuracy and the details of the simulation, where $\tau$ is the unit time (see Section 2.3 ). The integration time step for molecular fluids may be smaller. If bond lengths and angles are flexible, they are often responsible for the fastest modes in the system. If they are constrained, the 
maximum time step may depend on the constraint solver.

The various simulations presented in this thesis have been performed with different integrators. The reason for this is the fact that these integrators have different properties and are not all equally suitable for different types of simulations. An overview of the integrators and properties of the algorithms are given in this section.

\subsection{1 (Velocity) Verlet}

A well-known integration scheme in molecular dynamics simulations is the Verlet 62 ] scheme

$$
\mathbf{r}(t+\Delta t)=2 \mathbf{r}(t)-\mathbf{r}(t-\Delta t)+\mathbf{a}(t)(\Delta t)^{2}
$$

This scheme is derived from a Taylor series expansion of $\mathbf{r}$ around $t$ and has a discretization (truncation) error of $\mathcal{O}\left((\Delta t)^{4}\right)$. The Verlet scheme follows from subtracting the expansions for $\mathbf{r}(t-\Delta t)$ from that for $\mathbf{r}(t+\Delta t)$. Since the terms that have an odd power of $\Delta t$ cancel out, the accuracy of this scheme is an order higher than a simple Taylor series expansion up to the second time derivative of $\mathbf{r}$. The Verlet scheme does not include the integration of velocity, which is often calculated from the positions using a finite difference scheme. A more commonly used method, based on the Verlet scheme, is Velocity Verlet

$$
\begin{aligned}
\mathbf{r}_{i}(t+\Delta t) & =\mathbf{r}_{i}(t)+\mathbf{v}_{i}(t) \Delta t+\frac{1}{2} \mathbf{a}_{i}(t)(\Delta t)^{2} \\
\mathbf{v}_{i}(t+\Delta t) & =\mathbf{v}_{i}(t)+\frac{\mathbf{a}_{i}(t)+\mathbf{a}_{i}(t+\Delta t)}{2} \Delta t
\end{aligned}
$$

The Velocity Verlet scheme has a discretization error of $\mathcal{O}\left((\Delta t)^{3}\right)$ for the velocity, as opposed to an discretization error of $\mathcal{O}\left((\Delta t)^{2}\right)$ for the standard Verlet scheme with a central difference calculation for the velocity.

\subsubsection{Runge-Kutta}

Higher-order methods are sometimes desirable for enhanced accuracy. Such schemes could be constructed by including more terms in the Taylor series expansion, but this would require the calculation of higher derivatives of the force, which is computationally expensive. Alternatively, a single-step method can be devised that matches the accuracy of the higher-order Taylor series expansion by sequentially evaluating the function of interest $g$ at several points within the time increment $\Delta t$, instead of computing higher-order derivatives. Methods of this type are called Runge-Kutta methods. A large variety of such schemes exists. We only present the fourth-order 
explicit Runge-Kutta scheme

$$
\begin{aligned}
\mathbf{A}(t+\Delta t) & =\mathbf{A}(t)+\frac{1}{6}\left(\mathbf{k}_{1}+2 \mathbf{k}_{2}+2 \mathbf{k}_{3}+\mathbf{k}_{4}\right) \\
\mathbf{k}_{1} & =\Delta t g(t, \mathbf{A}(t)) \\
\mathbf{k}_{2} & =\Delta t g\left(t+\Delta t / 2, \mathbf{A}(t)+\mathbf{k}_{1} / 2\right) \\
\mathbf{k}_{3} & =\Delta t g\left(t+\Delta t / 2, \mathbf{A}(t)+\mathbf{k}_{2} / 2\right) \\
\mathbf{k}_{4} & =\Delta t g\left(t+\Delta t, \mathbf{A}(t)+\mathbf{k}_{3}\right)
\end{aligned}
$$

where $\mathbf{A}$ can represent, for example, positions $\mathbf{r}$ or velocities $\mathbf{v}$ of particles, and $g$ is the right-hand side of the governing first-order differential equation (e.g., equation of motion). Note that each increment in time requires four function evaluations. This makes the fourth-order scheme significantly more computationally expensive than lower-order schemes.

This scheme is called 'explicit' because each coefficient $\mathbf{k}_{i}$ depends on previously calculated coefficients and on function evaluations from the previous step $(\mathbf{r}(t), \mathbf{v}(t))$. Due to this feature, the method is easy to implement. However, the explicit RungeKutta scheme is only conditionally stable. Implicit Runge-Kutta schemes, on the other hand, are more difficult to implement, but they are more stable and much more accurate than explicit schemes.

In this thesis, the explicit fourth-order Runge-Kutta scheme is applied to the simulations related to Chapter 6 because it is accurate as well as a single-step algorithm. Single-step algorithms calculate the next information $(\mathbf{A}(t+\Delta t))$ based on the present $(\mathbf{A}(t))$, without needing prior information $(\mathbf{A}(t-\Delta t))$. This feature makes the algorithm 'self-starting', meaning that no additional algorithm is needed to start the integration. An accurate self-starting algorithm is required for the study of startup-flow (see Section 5.4.1).

\subsection{Non-bonded interactions between atoms}

In this section, we treat the interactions between atoms in systems where the no chemical bonds are formed, i.e., monoatomic gases, liquids and amorphous solids. Nonbonded interactions are typically weaker than bonded interactions, such as covalent or ionic bonds, and the number of interactions between any atom and its neighbors may vary between atoms and varies in time. We focus on simple monoatomic fluids. The atoms in these fluids are spherically symmetric, neutrally charged and do not exhibit chemical processes. Some examples of such fluids are Argon, Xenon and Krypton. The interactions between the atoms are described by an energy potential. Several potentials are known that can produce certain transport coefficients or phase transitions that are in good agreement with empirical findings. Thus, which potential to use depends on the system and on the quantities or phenomena of interest. 
The potential energy can be a function of the position of individual particles, and the relative position of particle pairs, triplets and even larger groups of simultaneously interacting particles

$$
U=\sum_{i=1}^{N} U\left(\mathbf{r}_{i}\right)+\sum_{i=1}^{N} \sum_{\substack{j=1 \\ j \neq i}}^{N} U\left(\mathbf{r}_{i}, \mathbf{r}_{j}\right)+\sum_{i=1}^{N} \sum_{\substack{j=1 \\ j \neq i}}^{N} \sum_{\substack{k=1 \\ k \neq i, j}}^{N} U\left(\mathbf{r}_{i}, \mathbf{r}_{j}, \mathbf{r}_{k}\right)+\ldots
$$

A suitable pair-interaction potential is known to be able to predict the properties of a simple fluid very accurately [63. Lee and Cummings [64] compared the shear viscosity calculated with a pair-interaction potential and a three-body interaction potential. They found that the three-body potential resulted only in a slightly lower viscosity over the range of shear rates reported. Furthermore, Marcelli et al. 65 67] have studied extensively the influence of three-body interactions on various quantities. They found a relation, independent of shear rate, between transport properties calculated with two-body and three-body interaction potentials. A correction could be applied to the energy, pressure and shear viscosity calculated with a two-body potential, rather than performing computationally expensive simulations with a three-body interaction potential.

We will only consider pair-potentials in this study. The value of these potentials are a function solely of the absolute distance between an interacting pair of atoms. The most common pair potential for simple fluids is the Lennard-Jones (LJ) potential 68

$$
U^{L J}=4 \epsilon\left[\left(\frac{\sigma}{r}\right)^{12}-\left(\frac{\sigma}{r}\right)^{6}\right],
$$

where $r=\left|\mathbf{r}_{i j}\right|=\left|\mathbf{r}_{i}-\mathbf{r}_{j}\right|$ is the absolute distance between atoms $i$ and $j, \epsilon$ is the well-depth of the potential and $\sigma$ the atomic length scale, which is chosen as the distance at which the function value is zero. This potential is strongly repulsive at short distances $\left(r<2^{1 / 6} \sigma\right)$ and attractive at longer distances $\left(r>2^{1 / 6} \sigma\right)$. The attractive part represents the Van der Waals forces between atoms. This term corresponds to the $6^{\text {th }}$ power in the potential, which is based on empirical findings. The repulsive interactions arise from Coulombic repulsions and, indirectly, from Pauli repulsion and the exclusion of electrons from regions of space where the orbitals of closed-shell atoms overlap. The repulsion corresponds to the $12^{\text {th }}$ power in the potential, which is chosen such that the power is related to that of the attractive term, which is convenient from a computational viewpoint.

The powers of the potential can also be chosen differently. Some powers that have been used in the literature are $(12,6),(9,6)$, and $(28,7)$, or more complicated versions, such as the $n-6$ LJ potential 69. However, the 12-6 LJ potential is by far the most commonly used potential for MD simulations of simple fluids. Fincham and Heyes [70] 
have compared the shear viscosity of experimental liquid Argon to that calculated from simulations of a LJ fluid. The authors found good agreement. Despite the simplicity of the LJ potential, different phases can be formed depending on the state point of the fluid (see also Figure 2.1). It has been shown that the phase diagram ${ }^{1}$ of a LJ fluid is in good agreement with experimental results [63, 71, 72.

The LJ potential is often truncated in order to reduce computation time. To prevent a discontinuity at the location where the potential is truncated, the whole potential is shifted down by the value of the potential at the point of truncation $U^{L J}\left(r_{c}\right)$, such that the truncated and shifted Lennard-Jones (LJTS) potential is given by

$$
U^{L J T S}= \begin{cases}4 \epsilon\left[\left(\frac{\sigma}{r}\right)^{12}-\left(\frac{\sigma}{r}\right)^{6}-\left(\frac{\sigma}{r_{c}}\right)^{12}+\left(\frac{\sigma}{r_{c}}\right)^{6}\right] & \text { for } r \leq r_{c} \\ 0 & \text { for } r>r_{c},\end{cases}
$$

where the cut-off distance is often chosen in the range $r_{c} \approx 2.5 \sigma \ldots 5 \sigma$. Truncating and shifting the Lennard-Jones potential can influence the transport properties of the fluid and its phase diagram [73 75]. Similarly, changing the repulsive power of the potential affect its transport properties and phase diagram [69, 76].

A special case of the truncated and shifted Lennard-Jones potential has been introduced by Weeks, Chandler and Anderson (WCA) [77. They have truncated the potential at the distance of the LJ potential energy minimum $r_{c}=2^{1 / 6} \sigma$ and shifted the remaining part to maintain a continuous potential energy function. By truncating at the deepest point of the LJ potential, the attractive part of the interaction is eliminated, leaving a purely repulsive potential, given by

$$
U^{W C A}=\left\{\begin{array}{ll}
4 \epsilon\left[\left(\frac{\sigma}{r}\right)^{12}-\left(\frac{\sigma}{r}\right)^{6}\right]+\epsilon & \text { for } r / \sigma \leq 2^{1 / 6} \\
0 & \text { for } r / \sigma>2^{1 / 6}
\end{array} .\right.
$$

The LJ, LJTS (with $r_{c}=2.5 \sigma$ ) and WCA potential are shown in Figure 2.2.

A shorter cut-off distance results in fewer interactions, which consequently results in a reduction of the computation time. Despite this obvious advantage of a short cut-off distance, the purely repulsive WCA potential has a limitation relative to a LJ potential that is truncated at a longer distance. Hansen and Verlet [71] showed that potentials with a repulsive and an attractive component are needed to reproduce a realistic phase diagram. Earlier attempts with purely repulsive potentials succeeded in predicting the phase transitions and the single phases, but did not manage to predict

\footnotetext{
${ }^{1}$ Since the number of atoms and the system volume are fixed by default, the density is a controlled quantity. Furthermore, the temperature of the fluid will be controlled in the simulations (see Section 2.5. When a phase diagram is mentioned in this thesis, this refers to a two-dimensional diagram with density and temperature on the axes, as this is the most natural choice for the simulations in this thesis.
} 


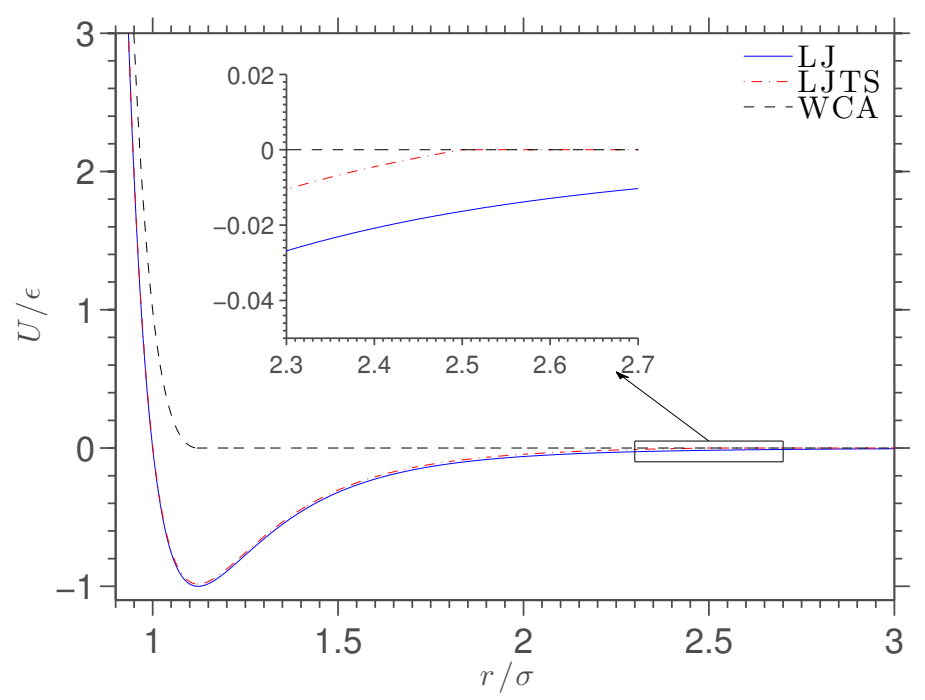

Figure 2.2: Three versions of the Lennard-Jones potential. The full LJ potential, the truncated and shifted Lennard-Jones (LJTS) potential at $r_{c}=2.5 \sigma$ and the WCA potential, which is truncated and shifted at $r_{c}=2^{1 / 6} \sigma$, which corresponds to the minimum of the LJ potential. 


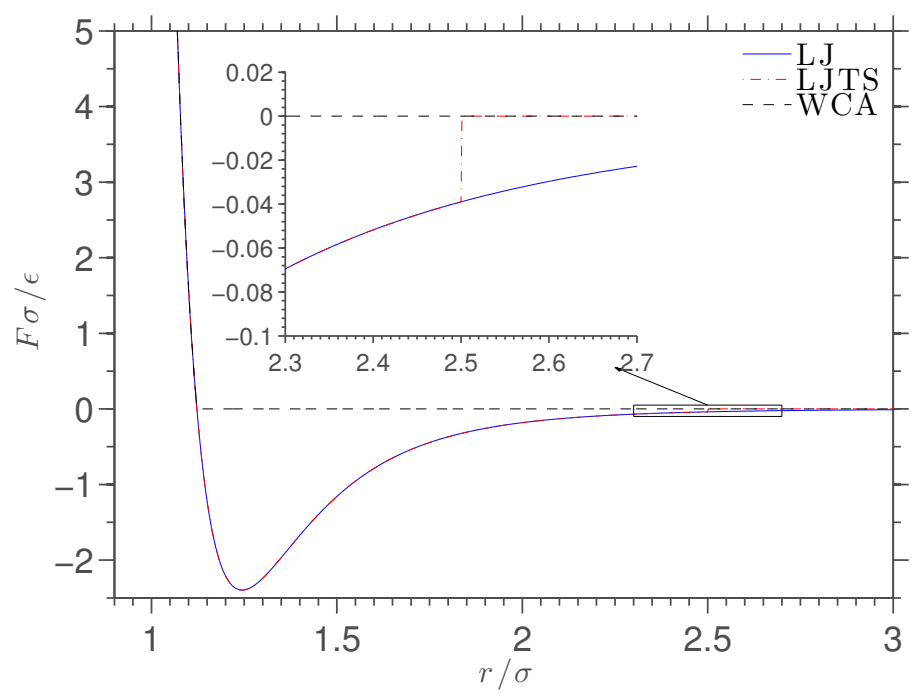

Figure 2.3: The interaction forces calculated from the potentials in Figure 2.2.

the coexistence of two fluid phases 78. Travis and Gubbins 79 compared several properties, like density, velocity and heat flux for a confined simple liquid simulated with a LJ and WCA potential. The authors found large differences in all properties for channels of widths $4 \sigma$ and $5.1 \sigma$. This was especially true for the narrowest of the two channels, in which the number of layers in the density profile were found to be dependent on the interaction potential used.

The force exerted on an atom due to interaction with another atom follows directly from the interaction potential as

$$
\mathbf{F}_{i j}=-\frac{d U}{d r} \frac{\mathbf{r}_{i j}}{r}
$$

where $\mathbf{F}_{i j}$ is the force acting on atom $i$ due to atom $j$ and $\mathbf{r}_{i j}=\mathbf{r}_{i}-\mathbf{r}_{j}$ is the contact vector. The scalar force $F=-d U / d r$ as a function of the distance between two atoms is shown in Figure 2.3. The force profiles are identical for distances smaller than the cut-off distance. This is because the shift of the potential has no influence on its slope. Since the WCA potential is truncated at the minimum of the LJ function, the corresponding force profile shows no discontinuity, as opposed to the force profile of the LJTS potential with any other cut-off distance than that of WCA. 


\subsection{Lennard-Jones Units}

MD simulations are often performed in reduced units. Since the characteristic scales are often very small in the conventional SI units, reduced units are not only more convenient for the user, but also avoid working in the vicinity of the numerical precision limitations of the computer. Each quantity is reduced with combinations of the length scale $\sigma$, the energy scale $\epsilon$ and the atomic mass $m$. The parameters that correspond to Argon are given in Table 2.1. While these parameters are known to be reasonably accurate for the reproduction of transport coefficients and phase transitions throughout the phase diagram, they do not always lead to the best possible agreement with some experimental measurements [71] and theoretical models [80. Parameters for other

Table 2.1: LJ parameters for liquid Argon, taken from Ref. 81.

Basic Units Symbol parameter for Argon

\begin{tabular}{lll}
\hline Length & $\sigma$ & $3.405 \times 10^{-10} \mathrm{~m}$ \\
Energy & $\epsilon / \mathrm{k}_{\mathrm{B}}$ & $119.8 \mathrm{~K}$ \\
& $\epsilon$ & $1.65 \times 10^{-21} \mathrm{~J}$ \\
Mass & $m$ & $6.69 \times 10^{-26} \mathrm{~kg}$
\end{tabular}

fluids can be found, for example, in Refs. [63, 72, 82, 83.

All dimensional quantities can be reduced to dimensionless quantities by means of these standard LJ units. To reduce a quantity $A$, with dimension $\mathrm{kg}^{\alpha} \mathrm{m}^{\beta} \mathrm{s}^{\gamma}$, one can write $A=A^{*} m^{\alpha+\gamma / 2} \sigma^{\beta+\gamma} \epsilon^{-\gamma / 2}$, where the asterisk denotes a non-dimensionalized quantity [84. The most relevant reductions for this work are listed in Table 2.2. The unreduced values of most quantities in Table 2.2 are very large or very small. These values are often inconvenient to work with and might in some cases even result in calculations with numbers of the same order as the machine precision. In simulations of simple fluids, one often only works with reduced quantities, which are chosen such that they are around the order of unity. They can then be converted back into real units at the end of the simulation.

\section{$2.4 \quad$ Pressure and stress tensors}

The hydrostatic pressure of a system in equilibrium is thermodynamically defined as

$$
p \equiv\left(\frac{\partial H}{\partial V}\right)_{N, T}
$$


Table 2.2: Reduced units of several quantities. The last column shows the physical values that correspond to unity in reduced units.

\begin{tabular}{lll} 
Variable & Reduced units & Real units \\
\hline Density & $\rho^{*}=\rho \sigma^{3} / m$ & $1678 \mathrm{~kg} / \mathrm{m}^{3}$ \\
Temperature & $T^{*}=T \mathrm{k}_{\mathrm{B}} / \epsilon$ & $119.8 \mathrm{~K}$ \\
Viscosity & $\eta^{*}=\eta \sigma^{2} / \sqrt{m \epsilon}$ & $9.076 \times 10^{-4}$ poise \\
Pressure & $p^{*}=p \sigma^{3} / \epsilon$ & $41.9 \mathrm{MPa}$ \\
Time & $t^{*}=t \sqrt{\epsilon /\left(m \sigma^{2}\right)}$ & $2.14 \times 10^{-12} \mathrm{~s}$ \\
Strain rate & $\dot{\gamma}^{*}=\dot{\gamma} \sqrt{m \sigma^{2} / \epsilon}$ & $4.66 \times 10^{11} \mathrm{~s}^{-1}$ \\
Force & $f^{*}=f \sigma / \epsilon$ & $4.9 \times 10^{-16} \mathrm{~N}$
\end{tabular}

where $H$ is the Helmholtz free energy $V$ the system volume, $N$ the number of particles and $T$ the temperature of the fluid, where the subscripts $N$ and $T$ represent fixed quantities. The thermodynamic definition of pressure is only valid in equilibrium and is inconvenient to calculate from a MD simulation. The mechanical interpretation of pressure is more common in MD

$$
p=-\lim _{\Delta A \rightarrow 0} \frac{\Delta \mathbf{F} \cdot \mathbf{n}}{\Delta A}=-\frac{d F_{n}}{d A},
$$

where $\mathbf{n}$ is unit vector normal to the surface and $A$ the surface area.

The hydrostatic pressure in a homogeneous fluid in equilibrium can be calculated with the virial equation

$$
p V=N \mathrm{k}_{\mathrm{B}} T+\frac{1}{3}\left\langle\sum_{i=1}^{N} \sum_{j \neq i} \mathbf{r}_{i j} \cdot \mathbf{F}_{i j}\right\rangle,
$$

where the first term on the right is the kinetic part, with $\mathrm{k}_{\mathrm{B}}$ Boltzmann's constant, and the second term the configurational part. Eq. 2.21) assumes isotropy of the fluid properties and thus is not valid for an inhomogeneous fluid or a fluid out of equilibrium.

The mechanical interpretation of the pressure $(\mathrm{Eq} 2.20)$ can easily be generalized to a position-dependent tensorial quantity. A pressure tensor can be defined from an infinitesimal force $d \mathbf{F}$ acting across an infinitesimal surface $d \mathbf{A}$, at location $\mathbf{r}$

$$
d \mathbf{F}(\mathbf{r}) \equiv-d \mathbf{A} \cdot \mathbf{P}(\mathbf{r})
$$

Similar to the virial equation, the pressure tensor can be split into a kinetic part $\mathbf{P}^{K}(\mathbf{r})=\mathrm{k}_{\mathrm{B}} T \rho(\mathbf{r}) \mathbf{I} / m$ due to convectional momentum transport, where $\mathbf{I}$ is the identity

\footnotetext{
${ }^{2}$ The Helmholtz free energy represents the amount of energy that can be transferred into work by a thermodynamic process.
} 
tensor, and a configurational part $\mathbf{P}^{U}(\mathbf{r})$ associated with the interactions between particles. The pressure tensor is given by $\mathbf{P}(\mathbf{r})=\mathbf{P}^{K}(\mathbf{r})+\mathbf{P}^{U}(\mathbf{r})$. Due to the different nature of both contributions, some extreme scenario's can be identified. In a dilute gas, the distances between atoms are generally much larger than in a liquid or solid. Hence, the number of interactions and the corresponding forces are relatively small and the configurational part of the pressure tensor will be small in comparison to the kinetic part. The opposite applies in a highly compressed dense solid/liquid, at moderate temperatures: the close packing results in large forces and thus a large configurational pressure tensor, whereas the transport of momentum due to fluctuations is relatively small. In a typical liquid, both terms are of the same order of magnitude and neither part can be neglected. Note, however, that the configurational pressure tensor depends strongly on the interaction potential. For example, by truncating the attractive part of a Lennard-Jones potential, the diagonal components of the configurational pressure tensor will increase in value and might even change from a negative to a positive value, depending on the state point of the fluid. This dependence on the potential is irrelevant for satisfying the continuum conservation equations since only the divergence of the pressure tensor occurs in these expressions.

The divergence of the pressure tensor can be derived from the evolution of the momentum density $\mathbf{J}(\mathbf{r})$ as

$$
\begin{aligned}
\frac{\partial \mathbf{J}(\mathbf{r})}{\partial t} & =\frac{\partial}{\partial t} \sum_{i-1}^{N} m_{i} \mathbf{v}_{i} \delta\left(\mathbf{r}-\mathbf{r}_{i}\right) \\
& =\sum_{i-1}^{N} m_{i} \dot{\mathbf{v}}_{i} \delta\left(\mathbf{r}-\mathbf{r}_{i}\right)-\nabla \cdot \sum_{i-1}^{N} m_{i} \mathbf{v}_{i} \mathbf{v}_{i} \delta\left(\mathbf{r}-\mathbf{r}_{i}\right) \\
& =\sum_{i-1}^{N} \mathbf{F}_{i} \delta\left(\mathbf{r}-\mathbf{r}_{i}\right)-\nabla \cdot \sum_{i-1}^{N} m_{i} \mathbf{v}_{i} \mathbf{v}_{i} \delta\left(\mathbf{r}-\mathbf{r}_{i}\right) \\
& =\frac{1}{2} \sum_{i-1}^{N} \sum_{j \neq i} \mathbf{F}_{i j}\left(\delta\left(\mathbf{r}-\mathbf{r}_{i}\right)-\delta\left(\mathbf{r}-\mathbf{r}_{j}\right)\right)-\nabla \cdot\left(\rho \mathbf{u u}+\sum_{i-1}^{N} \frac{\mathbf{p}_{i} \mathbf{p}_{i}}{m_{i}} \delta\left(\mathbf{r}-\mathbf{r}_{i}\right)\right) \\
& =-\nabla \cdot(\rho \mathbf{u u}+\mathbf{P}) .
\end{aligned}
$$

The fourth equality uses the fact that $\mathbf{F}_{i}=\sum_{j \neq i} \mathbf{F}_{i j}$, Newton's third law $\mathbf{F}_{i j}=-\mathbf{F}_{j i}$ and writes the velocity in terms of a fluctuation part and a streaming part $\mathbf{v}_{i}=$ $\mathbf{p}_{i} / m_{i}+\mathbf{u}\left(\mathbf{r}_{i}\right)$. Since the pair interaction forces are symmetric, the pressure tensor is by definition also symmetric. From the last equality, we can write

$$
\nabla \cdot \mathbf{P}(\mathbf{r})=\nabla \cdot \sum_{i-1}^{N} \frac{\mathbf{p}_{i} \mathbf{p}_{i}}{m_{i}} \delta\left(\mathbf{r}-\mathbf{r}_{i}\right)-\frac{1}{2} \sum_{i-1}^{N} \sum_{j \neq i} \mathbf{F}_{i j}\left(\delta\left(\mathbf{r}-\mathbf{r}_{i}\right)-\delta\left(\mathbf{r}-\mathbf{r}_{j}\right)\right) .
$$

This expression does not have a unique solution for the pressure tensor $\mathbf{P}(\mathbf{r})$, since it 
only defines the divergence of the pressure tensor.

In 1950, Irving and Kirkwood 85] pioneered an ingenious way to calculate the local microscopic pressure tensor from Eq. 2.24) by using a Taylor series expansion.

$$
\mathbf{P}(\mathbf{r})=\sum_{i=1}^{N}\left(\frac{\mathbf{p}_{i} \mathbf{p}_{i}}{m_{i}} \delta\left(\mathbf{r}-\mathbf{r}_{i}\right)+\frac{1}{2} \sum_{j \neq i} \mathbf{r}_{i j} \mathbf{F}_{i j} O_{i j}[\mathbf{r}] \delta\left(\mathbf{r}-\mathbf{r}_{i}\right)\right)
$$

where $\mathbf{r}_{i j}=\mathbf{r}_{i}-\mathbf{r}_{j}$ and the operator $O_{i j}$ follows from the integral over the following identity

$$
\begin{aligned}
\delta\left(\mathbf{r}-\mathbf{r}_{i}\right)-\delta\left(\mathbf{r}-\mathbf{r}_{j}\right) & =-\mathbf{r}_{i j} \cdot \frac{\partial}{\partial \mathbf{r}} \delta\left(\mathbf{r}-\mathbf{r}_{i}\right)+\frac{1}{2 !} \mathbf{r}_{i j} \mathbf{r}_{i j} \cdot \frac{\partial^{2}}{\partial \mathbf{r}^{2}} \delta\left(\mathbf{r}-\mathbf{r}_{i}\right)+\ldots \\
& =-\frac{\partial}{\partial \mathbf{r}} \cdot \mathbf{r}_{i j}\left(1-\frac{1}{2 !} \mathbf{r}_{i j} \cdot \frac{\partial}{\partial \mathbf{r}}+\ldots+\frac{1}{n !}\left(\mathbf{r}_{i j} \cdot \frac{\partial}{\partial \mathbf{r}}\right)^{n-1}+\ldots\right) \delta\left(\mathbf{r}-\mathbf{r}_{i}\right) \\
& =-\frac{\partial}{\partial \mathbf{r}} \cdot \mathbf{r}_{i j} O_{i j}[\mathbf{r}] \delta\left(\mathbf{r}-\mathbf{r}_{i}\right) .
\end{aligned}
$$

Their formulation is generally applicable for single-component atomic fluids in which the interactions between particles are described by a pair potential.

If the fluid is homogeneous (i.e., particles are homogeneously distributed over space), the Taylor series expansion reduces to $O_{i j}=1$ so that the pressure tensor is given by

$$
\mathbf{P}(\mathbf{r})=\sum_{i=1}^{N}\left(\frac{\mathbf{p}_{i} \mathbf{p}_{i}}{m_{i}} \delta\left(\mathbf{r}-\mathbf{r}_{i}\right)+\frac{1}{2} \sum_{j \neq i} \mathbf{r}_{i j} \mathbf{F}_{i j} \delta\left(\mathbf{r}-\mathbf{r}_{i}\right)\right),
$$

This result can be referred to as the IK1 pressure tensor [86. Since the pressure tensor for a homogeneous fluid is constant across the volume, the tensor can be averaged over the volume, resulting in

$$
\mathbf{P}=\frac{1}{V} \sum_{i=1}^{N}\left(\frac{\mathbf{p}_{i} \mathbf{p}_{i}}{m_{i}}+\frac{1}{2} \sum_{j \neq i} \mathbf{r}_{i j} \mathbf{F}_{i j}\right)
$$

When the spatial distribution of particles is inhomogeneous (for example near a solid interface), the expansion of $O_{i j}$ is required to calculate the local pressure tensor. An accurate approximation of $O_{i j}$ requires a large number of expansion terms, which are numerically expensive to calculate. Alternatively, $\delta\left(\mathbf{r}-\mathbf{r}_{i}\right)-\delta\left(\mathbf{r}-\mathbf{r}_{j}\right)$ can be expressed in terms of an integral over the path between the positions of the particles $i$ and $j$ 87 90.

$$
\delta\left(\mathbf{r}-\mathbf{r}_{i}\right)-\delta\left(\mathbf{r}-\mathbf{r}_{j}\right)=-\frac{\partial}{\partial \mathbf{r}} \cdot \mathbf{r}_{i j} \int_{0}^{1} \delta\left(\mathbf{r}-\mathbf{r}_{i}+\lambda \mathbf{r}_{i j}\right) d \lambda .
$$


The microscopic pressure tensor for an inhomogeneous fluid can be derived by substituting this identity into Eq. 2.24, which gives

$$
\mathbf{P}(\mathbf{r})=\sum_{i=1}^{N}\left(\frac{\mathbf{p}_{i} \mathbf{p}_{i}}{m_{i}} \delta\left(\mathbf{r}-\mathbf{r}_{i}\right)+\frac{1}{2} \sum_{j \neq i} \mathbf{r}_{i j} \mathbf{F}_{i j} \int_{0}^{1} d \lambda \delta\left(\mathbf{r}-\mathbf{r}_{i}+\lambda \mathbf{r}_{i j}\right)\right) .
$$

This formulation was first introduced by Schofield and Henderson [87, in 1982.

Mistura 91] derived the same expression for the pressure tensor in an inhomogeneous fluid in a different way. He argued that the path, over which the integral goes, is unambiguous as it comes only in the definition of the distance between two particles, which is uniquely defined. Harasima 92] developed another method that produces the same normal stress as the IK method, whereas the tangential stresses are different. While the local pressure tensors computed with both methods are not identical, due to a different distribution of the information, the surface tension calculated with both methods is the same. This means that the integral of the difference between normal and tangential stress across the channel is equal for both methods. Tsai 93] compared the virial equation (Eq. 22.21) to the IK method. He found that the tensorial method leads to more precise results when comparable efforts in computation are compared. Furthermore, the IK pressure tensor is more suitable out of equilibrium and for inhomogeneous fluids compared to the scalar virial equation (Eq. 2.21). Todd et al. 86] derived an algorithm, called the method of planes (MoP), that avoids the ambiguous choice of the interaction path. Local pressure components are computed from the consideration of interaction forces across a plane passing through the point of interest. This method, however, can only be used to calculate the shear stress and one of the diagonal components of the pressure tensor, rather than the full tensorial quantity. The authors compared, for a LJ fluid confined in a narrow slit pore, components of the pressure tensor, calculated with the MoP to those calculated with the IK1 approximation and to results based on the integration of the Navier-Stokes momentum balance equation, which does not require any atomistic information. This method is referred to as the IMC method. Good agreement was found between the MoP and IMC methods, while the IK1 approximation showed spurious oscillations. Another algorithm was introduced by Cormier et al. 94. They derived a pressure tensor based on averaging the local pressure tensor over a spherical volume. Recently, Heyes and coworkers [95] have shown, for the limiting case of infinitesimally thin bins, the equivalence between the MoP and the 'volume averaging' (VA) method, introduced by Cormier et al. 94].

A similar comparison as presented by Todd et al. [86, is shown in Figure 2.4 . We compare the IK1 approximation to the IK pressure for an inhomogeneous fluid (Eq. 2.30). Our simulations correspond to a LJ fluid with density $\rho=0.8$ and temperature $T=1.0$ in reduced units. The fluid is confined in the $x$-direction between 
two parallel atomistic walls, separated by a distance $W=11.1$ and the fluid is driven in the negative $y$-direction by a constant body force $f=0.2$.

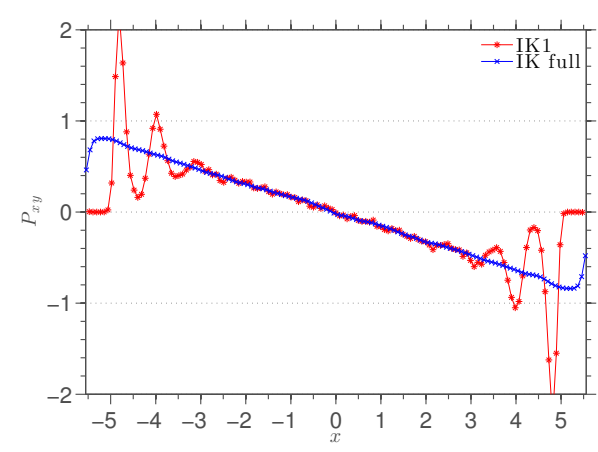

(a)

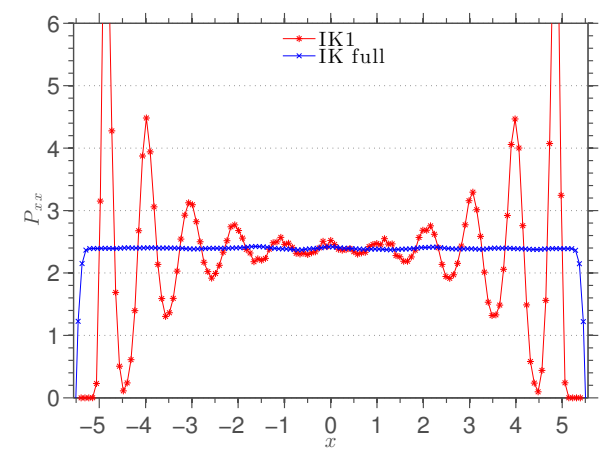

(b)

Figure 2.4: Shear stress $P_{x y}$ (a) and normal stress $P_{x x}$ (b) across the channel.

In recent years, the different pressure tensors have been extensively discussed and compared in the literature. For example, Sonne et al. [96] compared the IK and Harasima method for the calculation of the pressure tensor in a lipid bilayer in the liquid crystalline phase. The authors found a qualitative agreement between both methods. When a fluid is strongly layered, qualitative differences can arise between both definitions of the local pressure, as shown by Varnik et al. 97]. They compared the pressure tensors for a polymer film calculated with the IK, MoP and Harasima expressions. Hafskjold and Ikeshoji [98] compared the IK and the Harasima pressure tensors for a hard-sphere fluid. They concluded that the expressions are equal in Cartesian coordinates, but the Harasima method does not result in a correct pressure tensor in spherical coordinates.

The pressure tensor is defined as a compressive quantity, i.e., a positive pressure tensor is commonly associated with compressive (i.e., repulsive) forces. This means that positive diagonal terms contribute positively to the hydrostatic pressure.

The pressure tensor is (besides the sign convention in some literature) identical to the stress tensor $\boldsymbol{\sigma}$, which is more common in rheology and the solid mechanics literature 99, 100, whereas the pressure tensor is very common in MD. The literature is divided between the two tensorial quantities. Positive stresses are often associated with tensile forces, such that the relation between the pressure tensor and the stress tensor is given by $\boldsymbol{\sigma} \equiv-\mathbf{P}$. It must be noted that the stress tensor is sometimes also defined as a compressive quantity (for example in the field of geology), in which case it is identical to the pressure tensor. In this work, we only use the compressive definition 
for both quantities, such that $\boldsymbol{\sigma}=\mathbf{P}$.

The non-equilibrium pressure can be calculated from the pressure tensor as

$$
p=\frac{1}{3} \operatorname{tr}(\mathbf{P}) .
$$

Note that this is not by definition equal to the equilibrium pressure $p_{0}$, which is defined as one third of the trace of the pressure tensor for a fluid in equilibrium. Since the pressure tensor for a homogeneous fluid in equilibrium is isotropic, we can write $p_{0}=P_{x x}=P_{y y}=P_{z z}$. The difference between $p$ and $p_{0}$ due to deformation of the fluid is related to shear dilatancy [101, and will be studied in Chapter 5 .

\subsection{Thermostatting}

In many MD simulations, control of the fluid temperature is required. For example, when energy is added to the fluid, or when a change of temperature in time is desirable. The flow of a fluid results in an irreversible dissipation of mechanical energy into thermal energy, at a rate given by

$$
\dot{E}_{v}=-\int \mathbf{P}(\mathbf{r}): \nabla \mathbf{u}(\mathbf{r}) d^{3} \mathbf{r},
$$

which can be simplified to $\dot{E}_{v}=-V \mathbf{P}: \nabla \mathbf{u}$ for a homogeneous fluid.

In order to keep the temperature constant, a thermostat needs to remove heat at the same rate that it is created. The most efficient way to do this depends on the system of interest. If a system is fully periodic and the fluid is homogeneous, there are no gradients in temperature that would result in a conduction of heat in one direction, and there are no walls to extract the excess heat. Therefore, a homogeneous synthetic thermostat is needed [102]. Which thermostat to use becomes especially important far away from equilibrium (where the fluid can no longer locally be treated as if it were in equilibrium) or for the purpose of theoretical analysis, such as response theory. Furthermore, a thermostat can be applied to all particles equally, or only to a subset of the fluid. To date, unphysical phenomena are observed in simulations due to incorrect control of the temperature [49, 103, 104]. In order to discuss the control of temperature, first the temperature has to be defined.

In equilibrium, the thermodynamic definition of temperature is given by

$$
\frac{1}{T}=\left(\frac{\partial S}{\partial E}\right)_{V},
$$

where $T$ is the temperature, $S$ the entropy and $E$ the total energy of the system. By substituting the microcanonical phase-space distribution function in the Boltzmann 


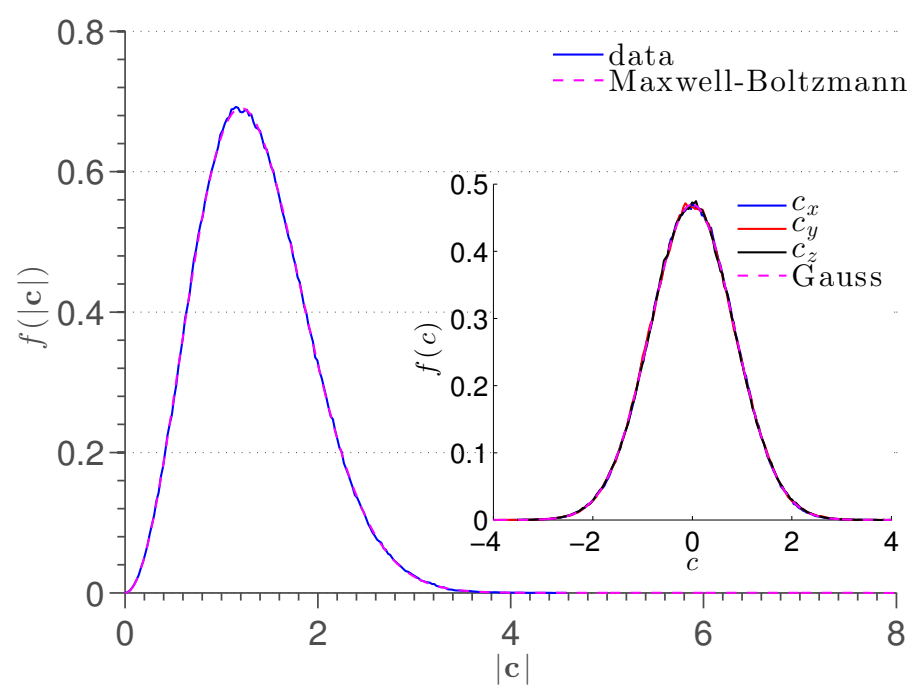

Figure 2.5: Histogram of the Maxwell-Boltzmann distribution of fluctuation speed and velocities (inset) for a fluid at temperature $T=0.722$.

entropy relation and taking the derivative with respect to $E$, it can be shown that in equilibrium, temperature is related to the kinetic energy

$$
\frac{3 N}{2} \mathrm{k}_{\mathrm{B}} T=\frac{1}{2} \sum_{i=1}^{N} m_{i} \mathbf{c}_{i} \cdot \mathbf{c}_{i} \equiv K,
$$

where $N$ is the number of atoms, $\mathbf{c}_{i}=\mathbf{p}_{i} / m_{i}$ the peculiar (or 'fluctuation') velocity, $\mathrm{k}_{\mathrm{B}}=1.38 \times 10^{-23}[\mathrm{~J} / \mathrm{K}]$ is Boltzmann's constant, $T$ the (kinetic) temperature and $K$ the fluctuation kinetic energy. This equation is called the equipartition theorem and defines the kinetic temperature. The average speed follows from Eq. 2.34) as $\langle|\mathbf{c}|\rangle=\sqrt{3 \mathrm{k}_{\mathrm{B}} T / m}$, assuming that all the particles have an equal mass. In reality, the speed of particles varies and if equipartition is true, the probability distribution of speed is described by the Maxwell-Boltzmann relation

$$
f(|\mathbf{c}|)=\sqrt{\frac{2}{\pi}\left(\frac{m}{\mathrm{k}_{\mathrm{B}} T}\right)^{3}}|\mathbf{c}|^{2} \exp \left(\frac{-m|\mathbf{c}|^{2}}{2 \mathrm{k}_{\mathrm{B}} T}\right) .
$$

Figure 2.5 shows the Maxwell-Boltzmann distribution of speed and velocity of atoms fluctuating in a fluid at a reduced temperature $T=0.722$. The inset shows the Gaussian distribution of fluctuation velocities in the $x, y$ and $z$ directions, where the mean of the Gaussian is zero and its variance $\mu=\mathrm{k}_{\mathrm{B}} T / m$.

Close to equilibrium, the equipartition theorem is approximately satisfied and the use of the kinetic temperature is permitted, whereas the thermodynamic definition 
is more difficult to evaluate and becomes questionable away from equilibrium. Most thermostats used in MD are based on controlling the thermal fluctuation velocities. Out of equilibrium, the fluctuation velocities $\mathbf{c}_{i}=\mathbf{v}_{i}-\mathbf{u}\left(\mathbf{r}_{i}\right)$ are given by the difference between the laboratory-frame velocities $\mathbf{v}_{i}$ and the streaming velocity $\mathbf{u}$. One can make an assumption for the streaming velocity profile, based on the superimposed constant rate of deformation. For example, for homogeneous shear flow in the linear regime, the velocity profile is $\mathbf{u}(\mathbf{r})=(\dot{\gamma} y, 0,0)$, where $\dot{\gamma}=\partial u_{x} / \partial y$ is the shear rate. A thermostatting approach based on the assumption of a known velocity profile is called a profile-biased thermostat (PBT). Evans and Morriss 28] noted that a PBT is a reasonable assumption only when the shear rate is small enough, such that no turbulence develops and the thermodynamic and kinetic temperature are almost identical. Far away from equilibrium it is, however, debatable which temperature must be controlled and what thermostat is most suitable to create a steady state without spurious phenomena [105, 106]. When the driving field is large, the velocity profile can become a nonlinear function of the position. Consequently, the PBT treats the deviations from the predicted linear profile as excess heat that needs to be extracted from the system. This attempt to restore a linear velocity profile may result in non-physical phenomena, such as the stabilization of the string phase. The string phase shows the formation of strings of particles in the direction of the flow, forming a hexagonal pattern in a plane parallel to the direction of the flow, as will be shown by means of a simulation in Section 2.5.3. This phenomenon was first observed by Erpenbeck [107, in 1984. Briefly it was unknown if the string phase was a physical phenomenon or an artifact of the simulations. Since the shear rates at which it is observed in simulations are far beyond the capabilities of experiments, neither possibility could easily be refuted. The answer to this question came when an alternative thermostatting method was developed [38.

Instead of assuming a linear velocity profile, the actual profile can be calculated during the simulation. The instantaneous steaming velocity profile is given by

$$
\mathbf{u}(\mathbf{r}) \equiv \frac{\sum_{i=1}^{N} m_{i} \mathbf{v}_{i} \delta\left(\mathbf{r}-\mathbf{r}_{i}\right)}{\sum_{i=1}^{N} m_{i} \delta\left(\mathbf{r}-\mathbf{r}_{i}\right)}
$$

Thermostats that use no prior assumptions about the streaming velocity profile and subtract a calculated profile from the laboratory velocities are referred to as profileunbiased thermostats (PUT) [38]. While there is no necessity to discretize space, the simulation cell is commonly divided into a finite number of subdomains, such as slabs (1D), bins (2D) or bricks (3D) in which the average velocity is calculated. This approach results in a discrete streaming velocity profile as a function of 1, 2 or 3 spatial 
directions, respectively. Another way to apply a PUT is to use a Fourier series expansion, this is, for example, done by Evans et al. [108]. A PUT is generally more difficult to apply and computationally more expensive than a PBT. Furthermore, caution is required in identifying the thermal and the streaming part of the instantaneous laboratory velocities. Studies on the occurrence of the string phase [49] have shown that a profile-unbiased thermostat (PUT) can be used to avoid the formation of a string phase.

In the following subsections, some of the most common thermostats are discussed. We will, however, briefly acknowledge a few other examples. In 1984, Berendsen et al. 109] developed a velocity rescaling algorithm that uses proportional feedback. The weak coupling of the particles to a heat bath allowed for fluctuations in the temperature. This method has major drawbacks: the thermostat does not generate or preserve any specific ensemble (see Section 4.2) and, despite its simplicity, it is not time-reversible. In addition to the deterministic thermostats that are common in MD, many stochastic thermostats exist, the most well-known of which are the Langevin thermostat [110] and the dissipative particle dynamics thermostat [11]. We only treat time-reversible deterministic methods methods in this work.

\subsubsection{Gaussian thermostat}

Woodcock 112 and Ashurst and Hoover 21] developed a velocity-rescaling method to keep the fluctuation kinetic energy exactly constant (i.e., isokinetic) in time, while the total energy is allowed to fluctuate.

The first deterministic isokinetic thermostat was later proposed by Hoover and Evans [43, 113. Instead of rescaling the velocities, their method added a thermostat term to the equations of motion, such that the evolution of the peculiar momentum (Eq. (2.4) ) for a fluid in equilibrium becomes

$$
\dot{\mathbf{p}}_{i}=\mathbf{F}_{i}-\zeta \mathbf{p}_{i}
$$

where $\zeta$ is a thermostat multiplier. The thermostat multiplier can have positive or negative values, depending on whether heat needs to be extracted or added, respectively.

Since the fluctuation kinetic energy is constrained, the instantaneous value of the 
thermostat multiplier in a multi-body system has to satisfy

$$
\begin{aligned}
\frac{\mathrm{d} K}{\mathrm{~d} t} & =\frac{\mathrm{d}}{\mathrm{d} t} \sum_{i=1}^{N} \frac{\mathbf{p}_{i} \cdot \mathbf{p}_{i}}{2 m_{i}} \\
& =\sum_{i=1}^{N} \frac{\mathbf{p}_{i} \cdot \dot{\mathbf{p}}_{i}}{m_{i}} \\
& =\sum_{i=1}^{N} \frac{\mathbf{p}_{i}}{m_{i}} \cdot\left\{\mathbf{F}_{i}-\zeta \mathbf{p}_{i}\right\} \\
& =0 .
\end{aligned}
$$

The thermostat multiplier is then given by

$$
\zeta=\frac{\sum_{i=1}^{N} \frac{\mathbf{p}_{i} \cdot \mathbf{F}_{i}}{m_{i}}}{\sum_{i=1}^{N} \frac{\mathbf{p}_{i} \cdot \mathbf{p}_{i}}{m_{i}}} .
$$

Evans et al. [114] noticed that this method is related to Gauss' principle of least constraint, which is the most fundamental theorem in physics according to Gauss, even more general than the laws introduced by Newton, Lagrange and Hamilton [115]. Due to the correspondence, the method became known as the Gaussian thermostat.

Since the thermostat multiplier is a function of the equations of motion, the functional form of Eq. 2.42 would be different for a system in which an external driving field is coupled to the fluid. The Gaussian thermostat is applied to a set of nonequilibrium equations of motion in Section 3.1.1.

The Gaussian thermostat constrains the fluctuation kinetic energy of the whole system, although individual particles still have various thermal velocities, as was shown in Figure 2.5. The thermal velocities in a thermostatted system sometimes satisfy welldefined distribution functions. Since this thermostat is derivable from a Hamiltonian [116, it can be proven that it generates a canonical ensemble (see Section 4.2). For a more detailed discussion, the reader is referred to some of the work done by William Hoover [58, 59].

Furthermore, note that the number of thermostatted particles $N$ does not necessarily have to be equal to the total number of particles in the system. In general, one could choose to thermostat either the walls (in case of a confined fluid), the fluid, or a combination. The thermostat can also be coupled to a subset of the fluid.

Instead of temperature, energy can be controlled through an ergostat. An ergostat mechanism can be devised based on Gauss' principle of least constraint [117. 


\subsubsection{Nosé-Hoover thermostat}

In 1984, Nosé [118] introduced a thermostatting mechanism based on the Nosé Hamiltonian

$$
\begin{aligned}
\mathcal{H}_{N}\left(\mathbf{r}, \mathbf{p}, s, p_{s}\right) & =\sum_{i=1}^{N} \frac{\mathbf{p}_{i} \cdot \mathbf{p}_{i}}{2 m_{i} s^{2}}+U+\frac{p_{s}^{2}}{2 Q}+\left(N_{f}+1\right) \mathrm{k}_{\mathrm{B}} T \ln s \\
& =\mathcal{H}_{0}\left(\mathbf{r}, \frac{\mathbf{p}}{s}\right)+\frac{p_{s}^{2}}{2 Q}+\left(N_{f}+1\right) \mathrm{k}_{\mathrm{B}} T \ln s,
\end{aligned}
$$

where $s$ is an added degree of freedom to the system, $p_{s}$ is the momentum of $s, Q$ is the Nosé mass and $N_{f}$ is the number of degrees of freedom in an $N$-body fluid. This method adds a degree of freedom to the system that needs to be controlled. This type of mechanism is called an extended system method. The existence of a Hamiltonian such as in Eq. 2.43 guaranteed the satisfaction of incompressibility of phase space (see Chapter 4).

From the Nosé Hamiltonian, we can derive a set of equations of motion

$$
\begin{aligned}
\dot{\mathbf{r}}_{i} & =\frac{\mathbf{p}_{i}}{m_{i}} \\
\dot{\mathbf{p}}_{i} & =\mathbf{F}_{i} \\
\dot{s} & =\frac{p_{s}}{Q} \\
\dot{p}_{s} & =\sum_{i=1}^{N} \frac{\mathbf{p}_{i} \cdot \mathbf{p}_{i}}{m_{i} s^{3}}-\frac{\left(N_{f}+1\right) \mathrm{k}_{B} T}{s} .
\end{aligned}
$$

Two additional equations of motion are thus introduced to control the temperature. This is known as Nosé's thermostat. Hoover and Hoover [119] noted that the Gaussian thermostat, presented in the previous subsection, is a special case of Nosé's thermostat.

Due to the rescaling of momenta, Nosé's thermostatting mechanism proved to be very impractical for the calculation of velocity autocorrelation functions. In order to circumvent this inconvenience, Nosé [120] suggested a coordinate transformation. Hoover 121 developed this idea further and rewrote the equations of motion to a more convenient form. This resulted in the thermostat that is now known as the Nosé-Hoover thermostat:

$$
\begin{aligned}
\dot{\mathbf{r}}_{i} & =\frac{\mathbf{p}_{i}}{m_{i}} \\
\dot{\mathbf{p}}_{i} & =\mathbf{F}_{i}-\zeta \mathbf{p}_{i} \\
\dot{\zeta} & =\frac{1}{Q}\left[\sum_{i=1}^{N} \frac{\mathbf{p}_{i} \cdot \mathbf{p}_{i}}{m_{i}}-N_{f} \mathrm{k}_{\mathrm{B}} T\right]
\end{aligned}
$$

The additional degree of freedom, $\zeta$, is Gaussian, with zero mean and a variance of $\left\langle\zeta^{2}\right\rangle=\mathrm{k}_{\mathrm{B}} T / M$, with $M=\sum_{i=1}^{N} m_{i}$ being the total mass of the fluid. The thermostat 

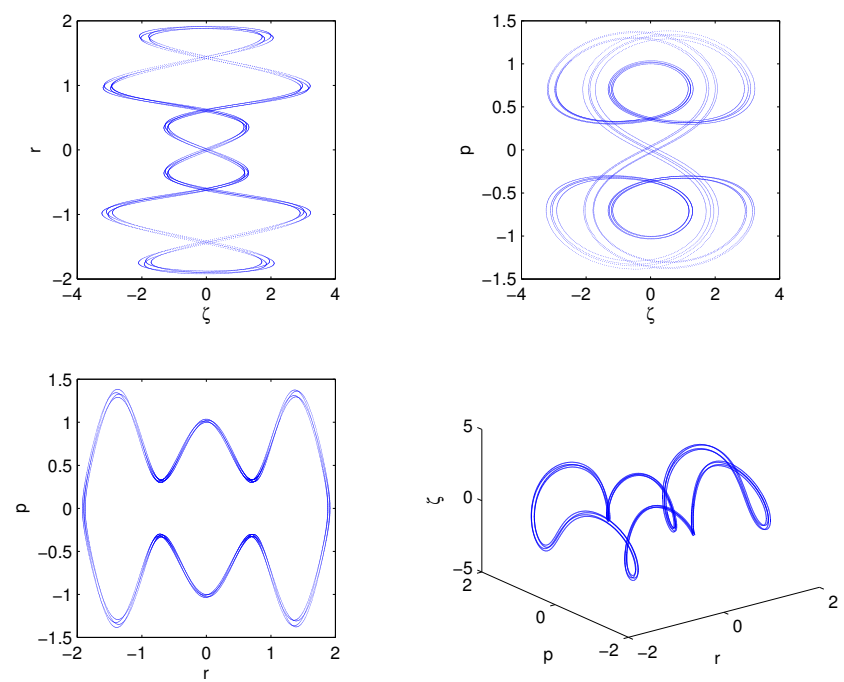

Figure 2.6: Single harmonic oscillator thermostatted with a Nosé-Hoover thermostat.

mass $Q$ determines how fast the thermostat should compensate the deviation from the target temperature. It must be chosen such that the system is critically damped. The value chosen for this parameter is critical in systems with a very small frequency spectrum (if chosen incorrectly, the velocity distribution can become incorrect or it can take a long time to reach the target temperature). However, since Lennard-Jones fluids have a wide spectrum, a wide range of values for $Q$ is permitted.

Figure 2.6 shows a simple example of a single harmonic oscillator controlled with the Nosé-Hoover thermostat. When the thermostat multiplier $\zeta$ is positive, heat is extracted from the system and heat is added to the system when $\zeta$ is negative. The thermostat multiplier is zero on average, because there is no external driving force. However, with the oscillators' velocity, also the thermostat multiplier oscillates in time, creating the Lissajous curve shown in the figure. Note that the energy of a single onedimensional un-thermostatted oscillator is simply given by the following Hamiltonian

$$
\mathcal{H}=\frac{p^{2}}{2 m}+\frac{1}{2} k r^{2}
$$

where $k$ is the stiffness. Hence, the equations of motion follow directly from the Hamiltonian and conservation of energy is guaranteed in the un-thermostatted case. The Nosé-Hoover formulation is not derivable from a Hamiltonian, in contrast to the Nosé thermostat. Consequently, it cannot be proven analytically that the thermostat generates a canonical ensemble. Examples are known for which a canonical ensemble is not generated, especially for very small or stiff systems, in which case the distribution 
function is non-canonical. Martyna et al. 122 constructed a chain of thermostats and showed that these do generate a canonical distribution for a harmonic oscillator.

Another extended-variable algorithm, based on Nosé's thermostat, is the NoséPoincaré thermostat [123. This scheme can be derived from a Hamiltonian and generates a canonical ensemble. It has not been widely applied in molecular dynamic simulations yet. Furthermore, a range of barostats (algorithms that control pressure) and ergostats (algorithms that control energy) are based on the same integral feedback method as the Nosé-Hoover thermostat. These algorithms are referred to as Nosé-Hoover barostats and ergostats, respectively.

\subsubsection{Braga-Travis configurational thermostat}

In some cases, for example when the driving field is very large, the velocity profile is not known a priori. One can still apply a kinetic thermostat using a PUT approach. This is, for example, done by Yong and Zhang [104] and by Todd and Daivis [124]. As mentioned previously, it can be difficult to distinguish between fluctuation velocities and streaming velocities, for example the streaming velocity profile in a boundarydriven flow is not known a priori and needs to be calculated on the fly in order to control the kinetic temperature. Alternatively, the positions of the atoms can be controlled via a configurational thermostat, based on the idea introduced by Rugh 125], in 1998. Shortly after, the first configurational thermostats were devised by Lue and Evans [126] in 2000 and via a different approach by Delhommelle and Evans [127] and Delhommelle et al. [128. Braga and Travis [129] modified the thermostat to a Nosé-Hoover-type configurational thermostat which is easier to implement. Based on the same idea, Braga and Travis [130] developed a configurational barostat, and later they devised a configurational thermostat for molecular fluids [131] in a very similar way as their atomic thermostat.

Only the Nosé-Hoover-type configurational thermostat presented by Braga and Travis is shown here. This algorithm is straight-forward to implement, as opposed to some of the alternative formulations.

$$
\begin{aligned}
\dot{\mathbf{r}}_{i} & =\frac{\mathbf{p}_{i}}{m_{i}}-\zeta \frac{\partial U}{\partial \mathbf{r}_{i}}, \\
\dot{\mathbf{p}}_{i} & =\mathbf{F}_{i}, \\
\dot{\zeta} & =\frac{1}{Q_{\eta}}\left[\sum_{i=1}^{N}\left(\frac{\partial U}{\partial \mathbf{r}_{i}}\right)^{2}-\mathrm{k}_{\mathrm{b}} T \sum_{i=1}^{N} \frac{\partial}{\partial \mathbf{r}_{i}} \cdot \frac{\partial U}{\partial \mathbf{r}_{i}}\right],
\end{aligned}
$$

where the Laplacian of the interaction potential for a LJ-type potential is given by

$$
\frac{\partial}{\partial \mathbf{r}_{i}} \cdot \frac{\partial U}{\partial \mathbf{r}_{i}}=\frac{\epsilon}{r^{2}}\left[528\left(\frac{\sigma}{r}\right)^{12}-120\left(\frac{\sigma}{r}\right)^{6}\right]
$$


Figure 2.7 shows a snapshot of a simulation in which a LJ fluid is sheared at a rate $\dot{\gamma}=5.0$, with the velocity in the $x$-direction and the gradient of velocity in $y$. The figure shows the difference in the microstructure between a fluid thermostatted by a kinetic and by a configurational thermostat. The density of the fluid is $\rho=0.8442$ and the temperature is kept constant at $T=0.722$ with a kinetic (profile-biased) NoséHoover thermostat in Figures 2.7(a) and 2.7(b) and a configurational Braga-Travis thermostat in 2.7(c) and $2.7(\mathrm{~d})$. The diameters of the LJ particles are shown at $30 \%$ of their actual length scale, to clearly show the structure in the fluid. The lighter atoms are located in the background, whereas the bright red atoms are in the foreground. Figures 2.7(a) and 2.7(b) shows that at least part of the atoms are arranged in a regular pattern. These patterns indicate the occurrence of the string phase, which vanish in Figures 2.7(c) and 2.7(d), where the configurational Braga-Travis thermostat is used.

\subsection{Periodic boundary conditions}

One goal of MD is to use numerical experiments to simulate and understand the rheological properties of fluids by mimicking real situations. However, the number of atoms in a real system is often many orders of magnitude larger than what is feasible in MD simulations. In simulations with periodic boundary conditions (PBC's), atoms do not only interact with their direct neighbors, but also with the periodic image of atoms on the other side of the domain, as is shown in Figure 2.8. The minimum image convention (MIC) states that a particle must interact only with the closest image of any other particle. This imposes a condition on the periodic simulation cell to be a least twice the cutoff length of the interaction potential. In case of various different types of interactions (for example in ionic liquids or in multi-component fluids) the longest interaction length is relevant. Electrostatic interactions often pose a problem since they are very long-ranged. Truncating these potentials at short distances would cause a large error, while making the simulation cell large enough for the potential to decay to small values means that the computational cost would become too large. Alternative methods are required in this case, such as Ewald summation [132] and related methods 133135$]$.

PBC's create the illusion of an infinite domain, making it possible to study bulk properties by simulating only a small number of atoms. However, real systems are always finite. The assumption that is made by using the PBC's is that the physical boundaries of a real system are far enough away to not influence the bulk behavior. This assumption is valid in most macroscopically large systems. For strongly confined fluids, this approximation does not necessarily hold. When a real system has a size of the same order of magnitude as the atomic length scale, the walls can result in an 


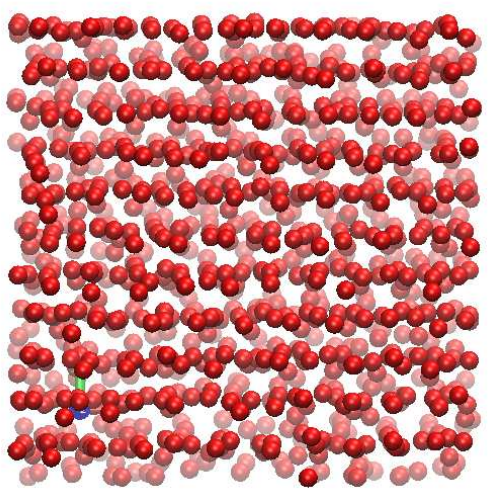

(a) Kinetic thermostat, $x-y$ plane

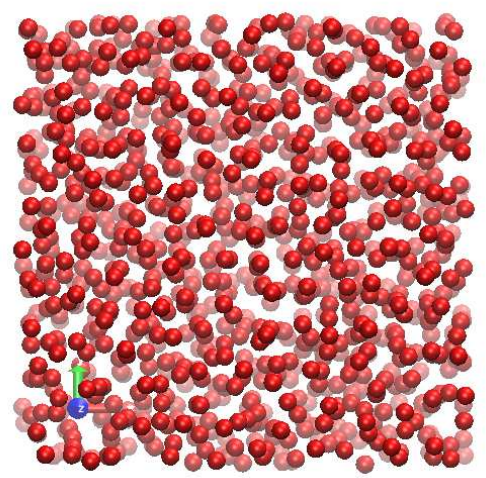

(c) Configurational thermostat, plane

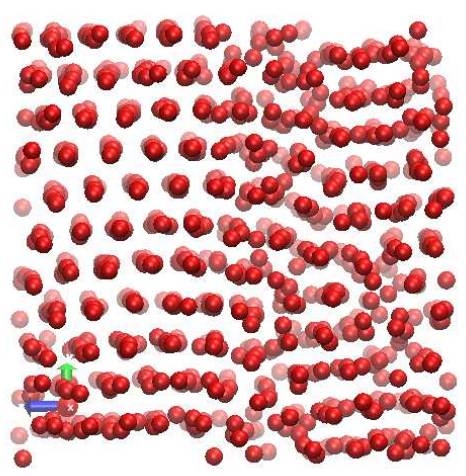

(b) Kinetic thermostat, $y$ - $z$ plane

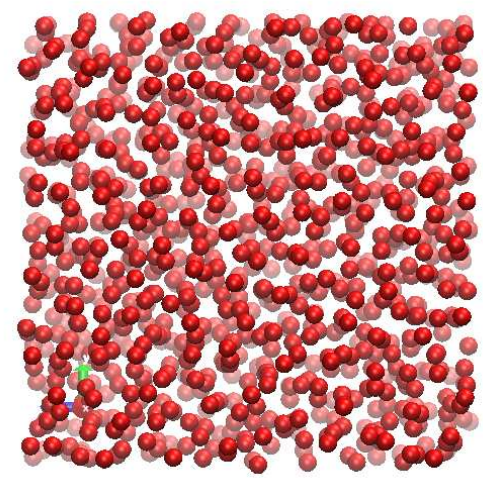

(d) Configurational thermostat, $y-z$ plane

Figure 2.7: Atomic shear flow at a shear rate $\dot{\gamma}=5.0$. The velocity is in the $x$-direction, with a gradient in $y$. The density of the fluid is $\rho=0.8442$ and the temperature is kept constant at $T=0.722$ with a kinetic (profile-biased) Nosé-Hoover thermostat in (a) and (b) and a configurational thermostat in (c) and (d). 


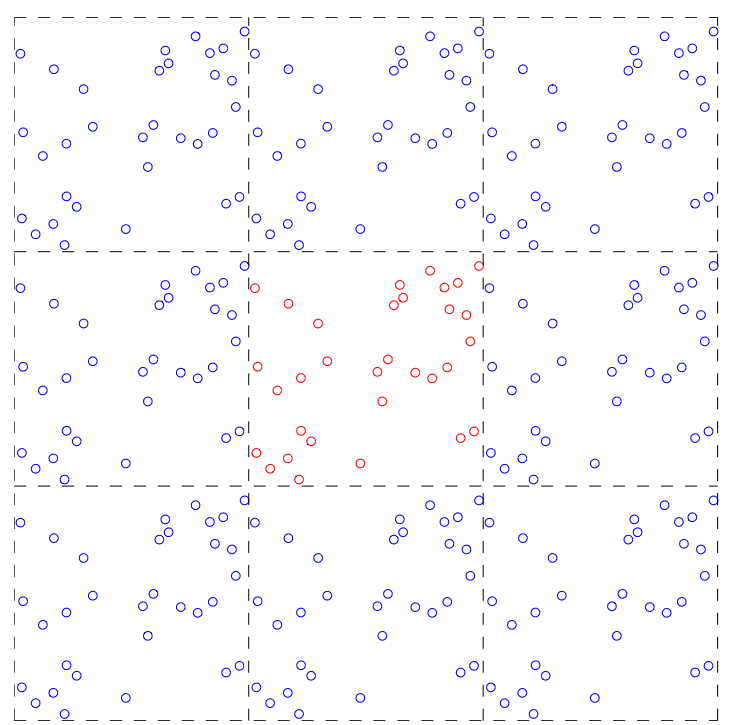

Figure 2.8: Standard periodic boundary conditions. The particles in the simulation cell are shown in red, while their mirror images are shown in blue.

inhomogeneous distribution of the atoms. In this case, walls must be simulated as well to study the near-wall behavior of the fluid, which deviates from the bulk behavior.

Liem et al. 37] tried to mimic real experiments by using thermostatted atomic walls to shear the fluid. Similar to experiments, the heat is transported to the walls and there removed by the heat bath. They compared their approach with homogeneous shear simulations. This approach can also be used to simulate flow through a channel where the walls are not sheared. The fluid can then be driven for example by: a thermal gradient, pressure gradient, body force or electrical current. Note that a large confined system can also be used to study bulk properties. This is, however, very inefficient since only the fluid far enough away from the wall can contribute to the bulk analysis. Furthermore, the interaction between the walls and the fluid affects quantities like the streaming velocity and the bulk density. The system needs to be sufficiently large in order to neglect the influence of the confinement on the bulk properties. It is for this reason that a homogeneous algorithm is more efficient.

Since periodic boundary conditions are not part of the physical system that is being mimicked, they should not disturb the flow in any way. A few considerations need to be made in order to choose proper boundary conditions. In the first place, a particle should not interact with its own periodic image. This becomes hard to avoid when interaction forces decay only weakly with distance, such as is the case with, for example, electrostatic interactions. If the simulation cell deforms under a 
certain flow field, care must be taken to ensure that the simulation cell never becomes too small in either direction 3 The challenges of a deforming simulation cell will be treated in Chapter3. Finally, it is important to appreciate that the configurational pressure tensor, even in a fluid that only has short-range interactions, can contain perturbations that have wave lengths longer than the size of the simulation cell. This effect is typically small, but can become important, for example when normal stress effects are studied. Similarly, the transport coefficients can, but should not, show a dependence on the size of the simulation cell. Yeh and Hummer [137] studied the size-dependence of the self-diffusion coefficient and the shear viscosity for water and a LJ fluid. They found that the diffusion coefficient increased with the system-size, whereas the shear viscosity showed no clear dependence on the system size. Schoen and Hoheisel [138, and Meijer et al. [139] have studied the shear viscosity for a LennardJones fluid near its triple point. They compared simulation results for various numbers of particles in the range from 108 to 4000. The authors concluded that no clear size dependence is observed when the number of particles is at least 256 .

We verify this finding for a WCA fluid at the state point $\rho=0.84, T=1.0$. We look at the shear viscosity and the normal stresses in a sheared fluid with a strain rate $\dot{\gamma}=0.02$. The number of particles ranges from $8^{3}=512$ to $24^{3}=13824$. Figure 2.9 shows the shear viscosity $\eta=-P_{x y} / \dot{\gamma}$ and the diagonal components of the pressure tensor as a function of the system's size. The data shows that the shear viscosity is not clearly dependent on the system size in the range of system sizes that were studied. The horizontal, red line in Figure 2.9(a) corresponds to the Green-Kubo shear viscosity $\eta_{0}=2.127$. Figure 2.9(b) shows that the difference in pressure $(p=\operatorname{tr}(\mathbf{P}) / 3)$ between the smallest and largest system is approximately $0.05 \%$ and thus generally considered to be negligible in most cases. The differences between the diagonal terms of the pressure tensor are even smaller than $0.05 \%$ of the pressure. Despite the small error bars, no consistency is seen in the magnitude of the stress differences, but also the order of the diagonal pressure terms varies. We know, from simulations at larger strain rates, that the diagonal components should satisfy $P_{z z} \leq P_{x x} \leq P_{y y}$, which is confirmed by the results shown in Ref. [28. This is however not always the case for the simulation results shown in Figure 2.9(b) due to statistical noise. Consequently, it is not possible to calculate meaningful normal stress differences from these MD simulation results.

\footnotetext{
${ }^{3}$ Correction terms exist to account for interactions with periodic images, see for example Ref. [136]. We do not consider such terms here since we primarily are concerned with short-range interactions.

${ }^{4}$ This should be the case at least for a WCA fluid at this state point. Simulations at lower densities have shown the opposite order between $P_{x x}$ and $P_{y y}$, while the value for $P_{z z}$ remains the smallest. Normal stress differences that confirm this image are shown in Appendix B (Figure B.2.
} 


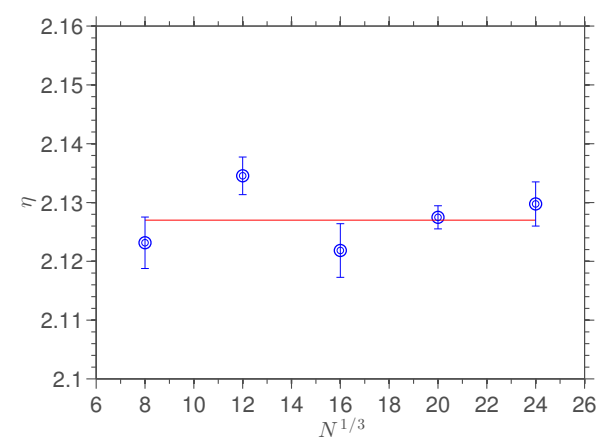

(a)

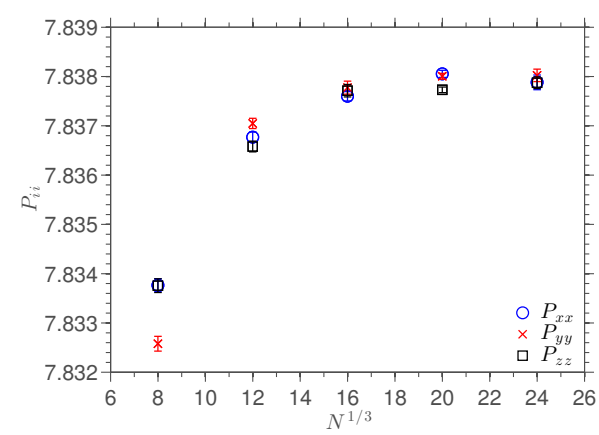

(b)

Figure 2.9: The shear viscosity and diagonal components of the pressure tensor in steady shear flow $(\rho=0.84, T=1.0, \dot{\gamma}=0.02)$ as a function of system size. The horizontal line in (a) corresponds to the Green-Kubo shear viscosity.

\subsection{Non-equilibrium molecular dynamics}

The equations of motion in the introduction of this chapter assumed that the trajectories of particles are described by Newton's equations of motion and that the fluid is in thermodynamic equilibrium. Thermodynamic equilibrium states that a system satisfies the combination of a mechanical, thermal and chemical equilibrium. In this thesis, we use the term thermodynamic equilibrium (or just 'equilibrium') to refer to a system that is not driven by any velocity field or force. Systems in equilibrium do not exhibit a net transport of mass, momentum and heat. Despite the absence of transport of these conserved quantities, the corresponding transport coefficients can be studied from the fluctuations of particles in a fluid in equilibrium. This was found by Lars Onsager [140, 141, who discovered that the relaxation to a steady state is closely coupled to the correlations between thermal fluctuations of atoms.

A more direct approach to study the transport in a fluid is by creating and maintaining a state out of thermodynamic equilibrium. This can be done by coupling an external force $\mathbf{F}^{E}$ to the fluid that drives a thermodynamic flux $\mathbf{J}_{i}$. For example, a temperature gradient causes the transport of heat, a velocity gradient leads to momentum flow and a gradient in concentration is associated with a current of mass. The field that is based on this approach is referred to as non-equilibrium molecular dynamics (NEMD) and was introduced in 1975 by Hoover and Ashurst [20].

In a non-equilibrium steady state, thermal and mechanical properties do not change with time. The non-equilibrium distribution function of a non-Hamiltonian system (e.g., a fluid that is thermostatted with the Nosé-Hoover thermostat) changes in time, 
both during the equilibration process and in the steady state, whereas the distribution function of a steady Hamiltonian system is constant in time. The work that is performed on a driven fluid is transformed into heat due to viscous dissipation. This increase in heat needs to be extracted from the system in order to reach a nonequilibrium steady state. In other words, the existence of a non-equilibrium steady state requires the presence of at least two links to the outside world, i.e., a source and a sink.

On a continuum scale we are dealing with macroscopically small volume elements. If the driving force is small enough such that the gradients of macroscopic fields are negligibly small, the system is said to be globally close to equilibrium and locally in equilibrium. When this is the case, equilibrium theories such as the Green-Kubo relations (see Section 4.4) are locally applicable.

Theoretical treatment of systems far from equilibrium is generally far more difficult than equilibrium and close-to-equilibrium systems. Processes and definitions out of equilibrium and especially far from equilibrium, are often far less well-understood than their equilibrium equivalent. This is especially applicable to some thermodynamic quantities such as temperature and entropy. Temperature is a well-defined quantity only in equilibrium. Also, a quantitative understanding of the entropy-production in NEMD is an open problem. These theoretical problems are not addressed here. The present work rather focusses on simulation methodology. Two NEMD approaches can be distinguished:

1. Imposing a flow field on a homogeneous fluid in a periodic simulation cell via a synthetic algorithm that couples an artificial driving force to the fluid.

2. Mimicking experimental systems in a natural way by modeling the geometry explicitly and driving the fluid via the boundaries or via an external force or gradient.

Either approach has some unique advantages over the other method, which justifies the importance that both approaches are studied and developed.

Synthetic methods provide the possibility to model a range of flow types and to study bulk fluids in a flexible way. Theoretical treatment, such as response theory, is possible due to the coupling of a force directly to the equations of motion that govern the dynamics of the fluid. Furthermore, only a relatively small number of particles is needed and since all state variables are independent of position, averaging over the volume is permitted. The synthetic algorithms allow for driving the fluid arbitrarily fast. This is not the case, for example, in boundary-driven confined fluids, where the maximum driving speed depends on the coupling between the wall and fluid. Slip poses a limitation on how fast the fluid can be driven. 
Phenomena such as layering of the atoms very close to a wall and slip between wall and fluid distinguish confined fluids from infinitely periodic homogeneous fluids. Confined-fluid simulations are needed if one is interested in studying, for example, lubrication of a very small bearing or the efficiency of micro-chip cooling.

Despite the differences between confined and unconfined fluid simulations, these types of simulation make use of many of the same tools and theories. Findings done with one type of simulation can often benefit the other, such that a symbiotic relationship can develop when they are studied together. 


\section{Homogeneous non-equilibrium molecular dynamics simulations}

As explained in Section 2.7, non-equilibrium molecular dynamics (NEMD) deals with fluids that are driven away from equilibrium by a force or a gradient (e.g., pressure, concentration, temperature or velocity). Driving a fluid via a gradient, a boundary or a solid object, can introduce an explicit dependence of structural and dynamical properties on the position. There are however methods to couple a driving force to a fluid in such a way that these properties are independent of the position. If this is the case, the fluid is homogeneous and averaging information over the volume is permitted.

These methods are known as synthetic [28], because they make use of an artificial driving force, that is not explicitly present in nature. This force is coupled to the fluid via the equations of motion, that govern the dynamics of all particles in the fluid.

Synthetic equations of motion in conjunction with an appropriate thermostat should result in spatially uniform properties, such that spatial averaging is permitted. The simulation cell generally deforms under the influence of the homogeneous deformation field. In order to satisfy the MIC (see Section 2.6), a remapping needs to be invoked to allow for indefinitely long simulations. The periodic boundary conditions, including a possible remapping, should not disturb the flow in any way. Suitable boundary conditions in combination with homogeneous equations of motion have only been found for a handful of flow types. These special cases are discussed in this chapter.

This chapter is organized as follows: In Section 3.1 the SLLOD equations of motion are introduced. Simple shear flow is briefly discussed in Section 3.2. Next, Planar elongational flow is presented in Section 3.3. and Section 3.4 discusses in detail a class 
of combined shear and elongational flows.

\subsection{Equations of motion}

We can extend the equations of motion from equilibrium molecular dynamics (Eqs. (2.3) and (2.4) by coupling an external driving force $\mathbf{F}^{E}$ to the fluid

$$
\begin{aligned}
\dot{\mathbf{r}}_{i} & =\frac{\mathbf{p}_{i}}{m_{i}}+\mathbf{C}_{i} \cdot \mathbf{F}^{E} \\
\dot{\mathbf{p}}_{i} & =\mathbf{F}_{i}-\mathbf{D}_{i} \cdot \mathbf{F}^{E},
\end{aligned}
$$

where $\mathbf{C}_{i}$ and $\mathbf{D}_{i}$ couple the force to the fluid. These tensors can be functions of the phase space vector $\boldsymbol{\Gamma}=\left(\mathbf{r}_{1}, \ldots, \mathbf{r}_{N}, \mathbf{p}_{1}, \ldots, \mathbf{p}_{N}\right)$, while the external force is independent of the phase space vector. Thus, all particles feel the same force at the same moment, which is required in order to maintain a system in which the density, temperature, pressure tensor and other quantities are constant across the volume. Because the driving force is explicitly present in the equations of motion, the system is amenable to theoretical treatment, such as response theory [142 144]. The response of many-body systems can be used to calculate transport coefficients.

Hoover et al. 25] have showed that adiabatic flows could be simulated by using the DOLLS Hamiltonian that couples a velocity gradient $\nabla \mathbf{u}$ to the fluid

$$
\mathcal{H}(\mathbf{r}, \mathbf{p})=\mathcal{H}_{0}(\mathbf{r}, \mathbf{p})+\sum_{i} \mathbf{r}_{i} \mathbf{p}_{i}:(\nabla \mathbf{u})^{T}
$$

where $\mathcal{H}_{0}$ is the Hamiltonian of a fluid at rest (Eq. 2.5)). The DOLLS equations of motion can be derived from this Hamiltonian

$$
\begin{aligned}
\dot{\mathbf{r}}_{i} & =\frac{\mathbf{p}_{i}}{m_{i}}+\mathbf{r}_{i} \cdot \nabla \mathbf{u}, \\
\dot{\mathbf{p}}_{i} & =\mathbf{F}_{i}-\nabla \mathbf{u} \cdot \mathbf{p}_{i} .
\end{aligned}
$$

Ladd 26] found that the DOLLS shear flow algorithm resulted in incorrect normal stress differences 11 Investigators, such as Ladd, Hoover, Evans and Morriss then developed the SLLOD equations of motion [26- 28, which are given by

$$
\begin{aligned}
\dot{\mathbf{r}}_{i} & =\frac{\mathbf{p}_{i}}{m_{i}}+\mathbf{r}_{i} \cdot \nabla \mathbf{u}, \\
\dot{\mathbf{p}}_{i} & =\mathbf{F}_{i}-\mathbf{p}_{i} \cdot \nabla \mathbf{u} .
\end{aligned}
$$

These equations of motion cannot be derived from a Hamiltonian. However, they can be derived from Newton's second law of motion as shown in Appendix A. Despite the

\footnotetext{
${ }^{1}$ The error that was made by the DOLLS formalism was later quantified by Evans and Morriss 27. via nonlinear-response theory.
} 
fact that the SLLOD equations of motion cannot be derived from a Hamiltonian, it is known that the (unthermostatted) SLLOD formalism satisfies the adiabatic incompressibility of phase space [28].

The evolution of an initial canonical distribution under SLLOD dynamics can be written as

$$
\frac{\partial f(t)}{\partial t}=-i \mathcal{L} f(t)=-\beta V \mathbf{P}(-t): \nabla \mathbf{u} f(t)
$$

where $-i \mathcal{L}$ is the Liouville operator (or $f$-Liouvillean) that acts on the $N$-body distribution function [28]. This differential equation has the following solution

$$
f(t)=\mathrm{e}^{-i \mathcal{L} t} f_{c}=\frac{\mathrm{e}^{-\beta V \mathbf{P}(-t): \nabla \mathbf{u}}}{\int \mathrm{e}^{-\beta \mathcal{H}_{0}} d \boldsymbol{\Gamma}},
$$

where $\exp (-i \mathcal{L} t)$ is the Liouville propagator.

In all cases considered here, a constant velocity gradient is instantaneously superimposed onto an equilibrium velocity distribution at time $t=0^{-}$, creating a local equilibrium distribution at time $t=0^{+}$. Once the system is in local equilibrium, the streaming motion is given by $\mathbf{u}(\mathbf{r})=\mathbf{r} \cdot \nabla \mathbf{u}$, such that the rate of change of position of all particles is described by

$$
\dot{\mathbf{r}}_{i}\left(t=0^{+}\right)=\dot{\mathbf{r}}_{i}\left(t=0^{-}\right)+\mathbf{r}_{i} \cdot \nabla \mathbf{u}
$$

After the streaming motion is imposed, a force is in certain cases needed to maintain the flow at times $t>0$.

A force $\mathbf{F}_{i}^{D}$ can be exerted on an equilibrium fluid to impose the velocity field and then maintain it [145]. This force is given by

$$
\mathbf{F}_{i}^{D}=m_{i} \mathbf{r}_{i} \cdot \nabla \mathbf{u} \delta(t)+m_{i} \mathbf{r}_{i} \cdot \nabla \mathbf{u} \cdot \nabla \mathbf{u} \Theta(t)
$$

where the first term on the right is the instantaneous perturbation needed to superimpose the flow field onto a fluid in rest and the second term maintains the flow. Applying this force to Newton's equations of motion for an equilibrium fluid and expressing the equations in terms of positions and peculiar momenta leads to the SLLOD equations of motion, as shown in Appendix A. For each flow field that satisfies $\nabla \mathbf{u} \cdot \nabla \mathbf{u}=0$, no constant external force is required to maintain the flow for $t>0$. An example of a flow type that satisfies this condition is simple shear flow (see Section 3.2.

By superimposing the velocity profile onto a fluid at rest, the equilibrium phase space distribution function (Eq. 4.5) ) is transformed into a local equilibrium distribution function given by

$$
f_{l}(\boldsymbol{\Gamma})=\frac{\mathrm{e}^{-\beta\left(\mathcal{H}_{0}+R\right)}}{\int \mathrm{e}^{-\beta \mathcal{H}_{0}} d \boldsymbol{\Gamma}}, \quad R=\sum_{i=1}^{N}\left\{\mathbf{p}_{i} \cdot \mathbf{r}_{i} \cdot \nabla \mathbf{u}+\frac{m_{i}}{2}\left(\mathbf{r}_{i} \cdot \nabla \mathbf{u}\right)^{2}\right\}
$$


This distribution is only valid at time $t=0^{+}$, before molecular relaxation occurs. For $t>0, f(\boldsymbol{\Gamma})$ evolves and becomes fractal if the fluid is thermostatted [146]. For shear flow, it has been proven [27] that propagating the local equilibrium distribution function with Newtonian dynamics is identical to propagating an initial canonical distribution function with the SLLOD algorithm, $f(t)=\exp (-i \mathcal{L} t) f_{c}=\exp \left(-i \mathcal{L}_{0} t\right) f_{l}$ where $i \mathcal{L}_{0}$ is the Newtonian $f$-Liouvillean.

The SLLOD equations of motion have a number of advantages over other MD approaches. Firstly, the state of the system is fully expressed in terms of positions and peculiar momenta. This is an advantage because the thermostat, internal energy and pressure tensor are often related to peculiar momenta rather than laboratory values. In methods that keep track of laboratory momenta, the streaming motion needs to be calculated and subtracted during the simulations in order to control quantities that are related to peculiar momenta. Calculating the streaming velocity profile from the laboratory momenta can lead to challenges, such as insufficient statistics or conceptional ambiguity, for example, when a velocity profile needs to be calculated as a function of all Cartesian directions [104], such that statistics is accumulated in small bricks and directional averaging is not permitted. Furthermore, calculation of the peculiar momenta can suffer from ambiguity when spatial smoothing is applied, as discussed in Ref. [147]. The SLLOD formalism does not pose these challenges, however, its use is limited to relatively simple velocity profiles. Another advantage of working with peculiar momenta is that they do not need to be altered when a particle crosses a periodic boundary, since the peculiar momenta are independent of position (otherwise the temperature would also be position dependent). Since the external field in synthetic equations of motion acts instantaneously on each atom, the response does not have to propagate through the system. This makes it possible to apply response theory and it allows for the study of time-dependent flow. Finally, the SLLOD equations of motion are exact arbitrarily far from equilibrium. In practice, it depends on the thermostatting mechanism how far from equilibrium the calculations are justified [31.

Hoover et al. 148, recently compared the DOLLS and SLLOD algorithms for shear flow to each other and to boundary-driven shear flow simulations. They found that the first normal stress differences computed with DOLLS and SLLOD were different, where SLLOD gives a better result than DOLLS. However, not only the equations of motion, but also the thermostat plays an important role and Hoover et al. 148, did not investigate this issue. 


\subsubsection{Thermostatted SLLOD}

When a driving field is coupled to the unthermostatted fluid, heat is created proportional to the rate of change of the internal energy

$$
\begin{aligned}
\dot{\mathcal{H}}_{0}(t) & =\frac{d}{d t} \sum_{i=1}^{N}\left(\frac{\mathbf{p}_{i} \cdot \mathbf{p}_{i}}{2 m_{i}}+U\left(\mathbf{r}_{i}\right)\right) \\
& =\sum_{i=1}^{N}\left(\frac{\mathbf{p}_{i} \cdot \dot{\mathbf{p}}_{i}}{m_{i}}-\dot{\mathbf{r}}_{i} \cdot \mathbf{F}_{i}\right) \\
& =\sum_{i=1}^{N}\left(-\frac{\mathbf{p}_{i} \cdot \mathbf{p}_{i}}{m_{i}} \cdot \nabla \mathbf{u}-\mathbf{r}_{i} \cdot \mathbf{F}_{i} \cdot \nabla \mathbf{u}\right) \\
& =-V \mathbf{P}(t): \nabla \mathbf{u},
\end{aligned}
$$

such that the internal energy subject to a constant flow field is given by

$$
\begin{aligned}
\mathcal{H}_{0}(t) & =\mathcal{H}_{0}(0)+\int_{0}^{t} \dot{\mathcal{H}}_{0}(s) d s \\
& =\mathcal{H}_{0}(0)-V \int_{0}^{t} \mathbf{P}(s): \nabla \mathbf{u} d s .
\end{aligned}
$$

Since the internal energy increases, also the kinetic temperature rises due to the external field. A thermostat is needed to fix the temperature as a constant of the motion. A kinetic thermostat can be coupled to the fluid as follows

$$
\begin{aligned}
\dot{\mathbf{r}}_{i} & =\frac{\mathbf{p}_{i}}{m_{i}}+\mathbf{r}_{i} \cdot \nabla \mathbf{u}, \\
\dot{\mathbf{p}}_{i} & =\mathbf{F}_{i}-\mathbf{p}_{i} \cdot \nabla \mathbf{u}-\zeta \mathbf{p}_{i},
\end{aligned}
$$

where $\zeta$ is the thermostat multiplier. The rate of energy dissipation in the thermostatted fluid is given by

$$
\dot{\mathcal{H}}_{0}=-V \mathbf{P}: \nabla \mathbf{u}-\zeta \sum_{i=1}^{N} \frac{\mathbf{p}_{i} \cdot \mathbf{p}_{i}}{m_{i}} .
$$

In order to reach and maintain a constant temperature, the thermostat multiplier must be such that the generated viscous heat is extracted from the system. One needs to carefully select a suitable thermostat for the given system. Two very common thermostats in NEMD are briefly discussed here: the Gaussian thermostat [28] and the Nosé-Hoover thermostat [121. The Gaussian thermostat formalism constrains the kinetic or internal energy to be exactly conserved. This leads to the isokinetic and isoenergetic ensemble, respectively. The latter is not treated here. The thermostat multiplier that is consistent with the SLLOD equations of motion can easily be derived, following the same approach as used in Section 2.5.1. Note that the fluctuation kinetic 
energy is not the same as the total kinetic energy out of equilibrium. Since the kinetic temperature is defined in terms of thermal fluctuations, the fluctuation kinetic energy is the controlled quantity

$$
\begin{aligned}
\frac{\mathrm{d} K}{\mathrm{~d} t} & =\frac{\mathrm{d}}{\mathrm{d} t} \sum_{i=1}^{N} \frac{\mathbf{p}_{i} \cdot \mathbf{p}_{i}}{2 m_{i}} \\
& =\sum_{i=1}^{N} \frac{\mathbf{p}_{i} \cdot \dot{\mathbf{p}}_{i}}{m_{i}} \\
& =\sum_{i=1}^{N} \frac{\mathbf{p}_{i}}{m_{i}} \cdot\left\{\mathbf{F}_{i}-\mathbf{p}_{i} \cdot \nabla \mathbf{u}-\zeta \mathbf{p}_{i}\right\} \\
& =0
\end{aligned}
$$

where we have substituted Eq. 3.19 for $\dot{\mathbf{p}}_{i}$. Using the last equality, we obtain the thermostat multiplier

$$
\zeta=\frac{\sum_{i=1}^{N} \frac{\mathbf{p}_{i}}{m_{i}} \cdot\left\{\mathbf{F}_{i}-\mathbf{p}_{i} \cdot \nabla \mathbf{u}\right\}}{\sum_{i=1}^{N} \frac{\mathbf{p}_{i} \cdot \mathbf{p}_{i}}{m_{i}}} .
$$

The fluctuation kinetic energy is exactly fixed to its initial value, without allowing for any fluctuations in the kinetic temperature. As opposed to its Nosé-Hoover equivalent, the Gaussian thermostat multiplier follows directly from the current state of the fluid. Combining Eqs. 3.14, 3.20 and 3.25, we can write

$$
\dot{\mathcal{H}}_{0}=-\sum_{i=1}^{N}\left(\mathbf{r}_{i} \cdot \mathbf{F}_{i} \cdot \nabla \mathbf{u}+\frac{\mathbf{p}_{i}}{m_{i}} \cdot \mathbf{F}_{i}\right) .
$$

This result shows that the internal energy dissipation of a Gaussian thermostatted fluid is related to the interaction forces (configurational contribution), rather than the kinetic temperature.

The Nosé-Hoover thermostat or the Braga-Travis configurational thermostat can be applied to the SLLOD equations of motion in an identical way as shown for an equilibrium fluid in Sections 2.5.2 and 2.5.3, respectively. Note that a combination of thermostats is also possible.

\subsubsection{Molecular SLLOD}

Most of this thesis deals with simple atomic fluids, however, the application of the SLLOD formalism to molecular fluids is briefly introduced, for the work presented in Section 6.4 Extending the SLLOD equations of motion from atomic to molecular 
fluids is relatively straight-forward: The primary difference is that the field is coupled to the center-of-mass momenta of the molecules, rather than that of each individual atom. Furthermore, additional forces are present between atoms that are connected by a bond. The molecular SLLOD equations of motion are given by

$$
\begin{aligned}
\dot{\mathbf{r}}_{i \alpha} & =\frac{\mathbf{p}_{i \alpha}}{m_{i \alpha}}+\mathbf{r}_{i} \cdot \nabla \mathbf{u}, \\
\dot{\mathbf{p}}_{i \alpha} & =\mathbf{F}_{i \alpha}+\mathbf{F}_{i \alpha}^{C}-\frac{m_{i \alpha}}{M_{i}} \mathbf{p}_{i} \cdot \nabla \mathbf{u}-\zeta^{M} \frac{m_{i \alpha}}{M_{i}} \mathbf{p}_{i},
\end{aligned}
$$

where $\mathbf{r}_{i \alpha}$ represents the position of site $\alpha$ in molecule $i, \mathbf{p}_{i \alpha}$ is the peculiar momentum of site $\alpha$ in molecule $i, \mathbf{r}_{i}=\sum_{\alpha=1}^{N_{b}} \mathbf{r}_{i \alpha} / N_{b}$ and $\mathbf{p}_{i}=\sum_{\alpha=1}^{N_{b}} \mathbf{p}_{i \alpha}$ are the center-of-mass position and peculiar momentum in a molecule with $N_{b}$ beads, $\mathbf{F}_{i \alpha}$ is the force due to non-bonded interactions with surrounding atoms, $\mathbf{F}_{i \alpha}^{C}$ is the force due to holonomic bond constraints (e.g., lengths, angles, torsions), $\zeta^{M}$ is the molecular thermostat multiplier, $m_{i \alpha}$ is the mass of site $\alpha$ in molecule $i$ and $M_{i}$ is the total mass of molecule $i$.

Alternatively, the evolution of a molecular fluid could also be described by the atomic SLLOD formalism, in which the driving field is coupled to the position and peculiar momentum of each atom, rather than the center-of-mass momenta of the molecules. It can be shown that both approaches result in the same steady state, since their phase space propagators lead to the same distribution function in the steadystate limit $f^{M}(t \rightarrow \infty)=f^{A}(t \rightarrow \infty)$ [149. However, the transient behavior of the fluid would not be the same since both algorithms result in a different local equilibrium state directly after superposing the streaming velocity profile onto the equilibrium fluid $f^{M}\left(t=0^{+}\right) \neq f^{A}\left(t=0^{+}\right)$.

Similarly to the driving field in the molecular SLLOD algorithm, also the molecular thermostat is coupled to the center-of-mass momenta of the molecules. Hence, a molecular thermostat controls the molecular kinetic temperature, which is not by definition identical to the atomic kinetic temperature. The molecular kinetic temperature of a thermostatted fluid is given by

$$
T^{M}=\frac{1}{\left(3 N_{m}-4\right) \mathrm{k}_{\mathrm{B}}} \sum_{i=1}^{N_{m}} \frac{\mathbf{p}_{i} \cdot \mathbf{p}_{i}}{M_{i}},
$$

where $N_{m}$ is the number of molecules and $3 N_{m}-4$ is the number of translational degrees of freedom of the center of mass of the molecules. In addition to the three degrees of freedom per molecule, an additional three degrees of freedom are suppressed due to the conservation of the total linear momentum and one more because the fluctuation kinetic energy is a constant of the motion. If the system is unthermostatted, the number of degrees of freedom would increase by one, to $3 N_{m}-3$. 
The molecular variant of a Gaussian thermostat is similar to Eq. (3.25), but now with the summation over the number of molecules and where the momenta are the center-of-mass values. It is important to note that the constraint forces do not appear in the expression for the thermostat multiplier because these internal constraint forces have a zero contribution to the center-of-mass properties of the molecules, $\sum_{\alpha} \mathbf{F}_{i \alpha}^{C}=\mathbf{0}$. Alternatively, an atomic thermostat could be applied to each atom in the molecular fluid. An atomic Gaussian thermostat would in fact include the constraint forces, since they have a non-zero effect on the individual atoms in each molecule.

Travis et al. [103, 131, 150, 151] have studied the application of thermostats to the molecular SLLOD formalism. They showed that the application of an atomic kinetic thermostat to a molecular fluid can lead to incorrect rotations of the molecules in a sheared fluid if the rotational degrees of freedom are not thermostatted correctly. The thermostat exerts a non-physical torque on the molecules if the rotational velocity profile is not calculated correctly, thus preventing the molecules from rotating in a natural way, proportional to the vorticity $\boldsymbol{\omega}=\frac{1}{2} \nabla \times \mathbf{u}$. Accurately calculating the rotational velocity profile is computationally expensive and often not practically feasible.

\subsection{Shear flow}

Planar Couette flow (PCF), or (simple) shear, is by far the most studied and wellunderstood type of flow. It is perhaps the simplest type of flow to study experimentally (e.g., lubrication), via boundary-driven simulations and also with a synthetic simulation approach. For this reason, many studies have been devoted to comparing computational results to experimental results, or to each other.

In shear flow, the streaming motion has a single direction of flow, and the streaming velocity has a gradient in a direction perpendicular to the flow. This is the shear rate $\dot{\gamma}$. The velocity gradient tensor for a sheared fluid with the flow in the $x$-direction and its gradient in $y$ is given by

$$
\nabla \mathbf{u}=\left[\begin{array}{ccc}
0 & 0 & 0 \\
\dot{\gamma} & 0 & 0 \\
0 & 0 & 0
\end{array}\right] .
$$

Substituting the velocity gradient (Eq. 3.30) into the equations of motion (Eqs. 3.18) and (3.19) gives

$$
\begin{aligned}
\dot{\mathbf{r}}_{i} & =\frac{\mathbf{p}_{i}}{m_{i}}+\dot{\gamma} y_{i} \mathbf{e}_{\mathbf{x}}, \\
\dot{\mathbf{p}}_{i} & =\mathbf{F}_{i}-\dot{\gamma} p_{y i} \mathbf{e}_{\mathbf{x}}-\zeta \mathbf{p}_{i},
\end{aligned}
$$

where $\mathbf{e}_{x}=(1,0,0)$. While the streaming motion is in the $x$-y plane, the simulations are fully three-dimensional, i.e., the positions, peculiar momenta and forces related to 


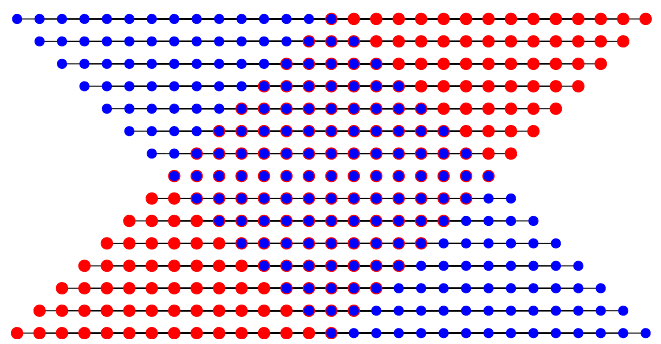

Figure 3.1: Typical reproducible lattice for shear flow. The lattice is reproducible after a time $\tau_{p}$, allowing for indefinitely long simulations. Blue indicates the initial lattice, red is the lattice after time $\tau_{p}$.

the particles are still three-dimensional. Reducing the system to a two-dimensional problem would change the properties of the fluid.

Suitable periodic boundary conditions are needed, such that particles that leave the primitive cell, re-enter with the appropriate momentum to maintain an undisturbed flow. The Lagrangian-Rhomboid periodic boundary is a common, but not unique, way to take care of the boundaries. These boundary conditions allow the simulation cell to evolve according to the streamlines of the flow. By doing so, the lattice vectors that span the cell become dependent on the velocity gradient

$$
\dot{\mathbf{L}}_{\alpha}(t)=\mathbf{L}_{\alpha}(t) \cdot \nabla \mathbf{u}, \quad \alpha=1,2,3
$$

The deformation of the simulation cell is limited by the minimum image convention (MIC), as explained in Section 2.6. The lattice vectors can easily be reset to their initial values after a time $\tau_{p}=\dot{\gamma}^{-1}$ and the particle positions are mapped back into the primitive cell simply by applying the periodic boundary conditions. This process is repeated after every time $\tau_{p}$ and can be done infinitely many times. An example of an initial and final lattice are shown in Figure 3.1. The corresponding initial lattice vectors corresponding to the figure are given by

$$
\mathbf{L}_{1}=(1,0,0), \quad \mathbf{L}_{2}=(-1 . / \sqrt{2}, 1 / \sqrt{2}, 0), \quad \mathbf{L}_{3}=(0,0,1)
$$

Applying Eq. 3.33 gives for the evolution of $\mathbf{L}_{2}$ as a linear function of time

$$
\mathbf{L}_{2}(t)=\mathbf{L}_{2}(0)+\dot{\gamma} t L_{2_{y}}(0) \mathbf{e}_{x}
$$

whereas the other lattice vectors $\mathbf{L}_{1}$ and $\mathbf{L}_{3}$ do not change in time. In this example, the initial and final angle between lattice vectors $\mathbf{L}_{1}$ and $\mathbf{L}_{2}$ are $135^{\circ}$ and $45^{\circ}$, respectively. 


\subsection{Elongational flows}

Elongational (or 'extensional') flows are shear-free flows that are caused by a contraction, extension, or a combination of both. Such flows occur in several industrial (for example extrusion and moulding processes) and biological processes, but are generally difficult to study with computer simulations or real experiments. If a fluid element is stretched at a constant rate of deformation, the actual displacement increases exponentially with time. Hence, a fluid sample in an elongational experiment quickly becomes very long or thin. Similarly, the evolution of the simulation cell is described by the streaming motion of the fluid, which also increases or decreases exponentially with time. This can pose a challenge in simulations since the minimum size of a simulation cell is limited by the MIC and the finite size of particles. In order to simulate elongational flows indefinitely long while satisfying the MIC, special boundary conditions are required. Such boundary conditions are treated in this section.

Volume preserving $\left(\operatorname{tr}(\nabla \mathbf{u})=\frac{\Delta V}{V}=0\right)$ shear-free flows have a velocity gradient that satisfies the following form

$$
\nabla \mathbf{u}=\left[\begin{array}{ccc}
\dot{\epsilon} & 0 & 0 \\
0 & -\dot{\epsilon}(1+b) / 2 & 0 \\
0 & 0 & -\dot{\epsilon}(1-b) / 2
\end{array}\right]
$$

where $\dot{\epsilon}$ is the rate of elongation and the variable $b$ determines the type of shear-free flow. The eigenvalues of the velocity gradient are given by $\lambda_{1}=\dot{\epsilon}, \lambda_{2}=\dot{\epsilon}(b-1) / 2$ and $\lambda_{3}=-\lambda_{1}-\lambda_{2}=-\dot{\epsilon}(b+1) / 2$. Since the trace of the velocity gradient is zero, there are only two independent eigenvalues. A few special cases can be identified in terms of the velocity gradient: uniaxial stretching flow (USF) when $b=0$ and $\dot{\epsilon}>0$, planar elongational flow (PEF) when $b=1$ and $\dot{\epsilon} \neq 0$, and biaxial stretching flow (BSF) when $b=0$ and $\dot{\epsilon}<0$. Planar elongational flow is discussed in more detail in this section.

\subsubsection{Planar Elongational Flow}

In planar elongational flow, a fluid is contracted in one direction and stretched at the same rate $\dot{\epsilon}$ in another (perpendicular) direction. The velocity gradient for a fluid that is stretched along the $x$-direction and contracted in $y$ is given by

$$
\nabla \mathbf{u}=\left[\begin{array}{ccc}
\dot{\epsilon} & 0 & 0 \\
0 & -\dot{\epsilon} & 0 \\
0 & 0 & 0
\end{array}\right]
$$




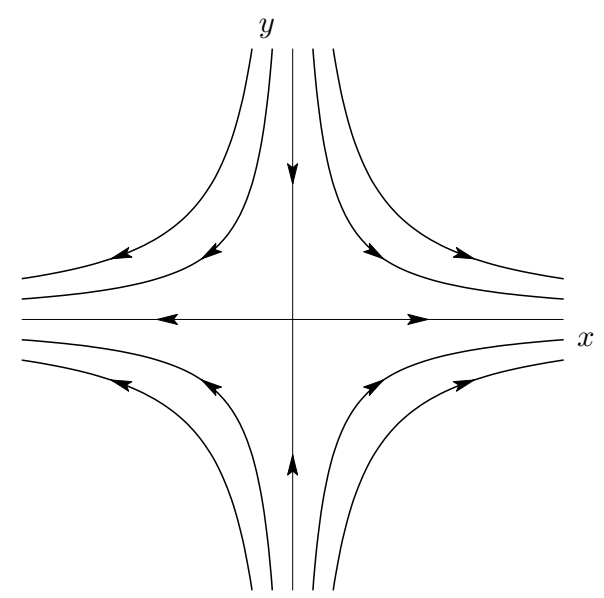

Figure 3.2: Streamlines for PEF with the extension direction along the $x$-axis and the contraction directions along the $y$-axis.

Substituting the velocity gradient for PEF into the SLLOD equations of motion (Eqs.3.18 and 3.19) gives the evolution of the positions and momenta of particles

$$
\begin{aligned}
\dot{\mathbf{r}}_{i} & =\frac{\mathbf{p}_{i}}{m_{i}}+\dot{\epsilon}\left(x_{i} \mathbf{e}_{\mathbf{x}}-y_{i} \mathbf{e}_{\mathbf{y}}\right), \\
\dot{\mathbf{p}}_{i} & =\mathbf{F}_{i}-\dot{\epsilon}\left(p_{x i} \mathbf{e}_{\mathbf{x}}-p_{y i} \mathbf{e}_{\mathbf{y}}\right)-\zeta \mathbf{p}_{i},
\end{aligned}
$$

where $\mathbf{e}_{\mathbf{y}}=(0,1,0)$.

Typical streamlines for PEF are shown in Figure 3.2 PEF leads to streaming motion in the $x$ - $y$-plane. The evolution of the center-of-mass position $(x, y)$ and the total momentum $\left(p_{x}, p_{y}\right)$ of the system of particles can be described by the following expressions

$$
\begin{aligned}
\dot{x}(t) & =\dot{\epsilon} x(t), \\
\dot{y}(t) & =-\dot{\epsilon} y(t), \\
\dot{p}_{x}(t) & =-\dot{\epsilon} p_{x}(t), \\
\dot{p}_{y}(t) & =\dot{\epsilon} p_{y}(t) .
\end{aligned}
$$

These expressions all have a formal solution of the following form $A(t)=A(0) \exp ( \pm \dot{\epsilon} t)$. This not only illustrates the fact that the simulation cell contracts exponentially with time in the $y$-direction, but it also states that an initial non-zero momentum of a the system $p_{y}(0)$ grows exponentially with time. Care must be taken in extracting the non-zero total momentum from the system during a simulation. Similarly, a non-zero $x$-component of the center-of-mass position would grow exponentially in time, but this has no effect on the simulation due to the periodic boundary conditions [124]. 


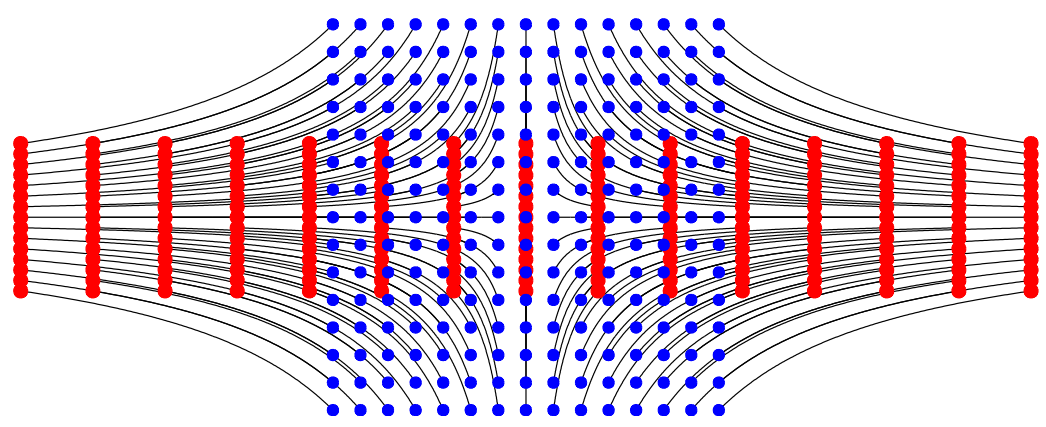

Figure 3.3: The square lattice is compressed in one direction and extended in another. The initial lattice is shown in blue and the lattice after time $\tau_{p}$ is shown in red. The black lines are the streamlines of the lattice sites.

\subsubsection{Kraynik-Reinelt Periodic boundary conditions}

Heyes [152] was the first to perform NEMD simulations of various types of elongational flows in 1985. However, the simulation time was limited by the lack of reproducible boundary conditions and not all simulations reached a steady state. If a cubic simulation cell deforms under PEF, the cell grows in the $x$-direction and shrinks in the $y$-direction, as shown for a square lattice in Figure 3.3 . The initial lattice is shown in blue and a deformed lattice is shown in red. The black lines are the streamlines of the lattice sites. The deformation in a NEMD simulation would be limited by the MIC, which states that the length of the simulation cell in the direction of each lattice vector needs to be at least twice the cutoff radius $r_{c}$ of the interaction potential. Thus, the maximum simulation time is given by

$$
t_{\max }=\frac{1}{\dot{\epsilon}} \ln \left(\frac{2 r_{c}}{L_{y}(0)}\right)
$$

where $L_{y}(0)$ is the initial length of the simulation cell in the direction of contraction. The deformation could continue indefinitely if a situation could be achieved in which the lattice is reproducible, such that it could be remapped to the original shape and size. In other words, special compatible and reproducible boundary conditions are required in order to allow for indefinitely long simulation times. Kraynik and Reinelt 153. devised periodic boundary conditions that are suitable for PEF. Furthermore, they have proven that $\mathrm{PEF}$ is the only elongational flow for which such boundary conditions can exist.

A reproducible lattice exists only if the following condition is satisfied

$$
\mathbf{L}_{i}\left(t=\tau_{p}\right)=\mathbf{L}_{i}(0) \cdot \boldsymbol{\Lambda}\left(\tau_{p}\right)=N_{i 1} \mathbf{B}_{1}+N_{i 2} \mathbf{B}_{2}+N_{i 3} \mathbf{B}_{3}=\mathbf{L}_{i}(0)
$$


where $\tau_{p}$ is the time at which the lattice is reproduced, $\mathbf{B}_{i}$ are orthogonal basis vectors, $N_{i j}$ are elements of an integer matrix and $\boldsymbol{\Lambda}(t)=\exp (\nabla \mathbf{u} t)$ describes the evolution of the lattice continuously in time as

$$
\mathbf{L}_{i}(t)=\mathbf{L}_{i}(0) \cdot \boldsymbol{\Lambda}(t)
$$

Reproducibility requires the existence of an integer matrix $\mathbf{N}$ that satisfies Eq. 3.45 at a finite time $\tau_{p}$. In order to find such a matrix the following eigenvalue problem has to be solved

$$
\left(\mathbf{N}-\lambda_{i} \mathbf{I}\right) \cdot \mathbf{v}_{i}=\mathbf{0}
$$

where $\mathbf{I}$ is the unit tensor, $\lambda_{i}=\exp \left((\nabla \mathrm{u})_{i i} \tau_{p}\right)$ an eigenvalue of $\mathbf{N}$, and $\mathbf{v}_{i}$ its corresponding eigenvector.

In order to calculate the remapping time $\tau_{p}$ of the lattice, first the characteristic equation from the eigenvalue problem in Eq. 3.47) needs to be solved

$$
\lambda_{p}=\frac{k \pm \sqrt{k^{2}-4}}{2}, \quad k=3,4,5, \ldots
$$

and defining $\lambda_{p} \equiv \exp \left(\epsilon_{p}\right)$, with the Hencky strain $\epsilon_{p}=\dot{\epsilon} \tau_{p}=\ln \left(\lambda_{p}\right)$, hence $\tau_{p}=$ $\ln \left(\lambda_{p}\right) / \dot{\epsilon}$. The solution of Eq. (3.48) depends on the value of an integer quantity $k \geq 3$, that can be chosen. Substituting the chosen value for $k$ in combination with a chosen positive integer $N_{11}$ into the following equation

$$
N_{12}=-\sqrt{N_{11}\left(k-N_{11}\right)-1}
$$

results in a value for $N_{12}$. A reproducible lattice can be constructed if $N_{12}$ has an integer value.

Once the existence of a reproducible lattice has been ensured, the initial (cubic) lattice can be constructed. The basis vectors $\mathbf{B}_{1}$ and $\mathbf{B}_{2}$ are oriented in the $x-y$ plane, whereas $\mathbf{B}_{3}$ is oriented parallel to the $z$-axis, orthogonal to $\mathbf{B}_{1}$ and $\mathbf{B}_{2}$. Furthermore, the direction of $\mathbf{B}_{1}$ is given by a 'magic angle' $\theta$ with respect to the $x$-axis. This angle depends on the previously used values for $k$ and $N_{11}$ and is given by

$$
\theta=\tan ^{-1}\left(\frac{N_{11}-\lambda_{p}}{N_{12}}\right)
$$

The basis vectors $\mathbf{B}_{i}$ of the initial lattice are given by

$$
\begin{aligned}
& \mathbf{B}_{1}=(\cos (\theta), \sin (\theta), 0), \\
& \mathbf{B}_{2}=(-\sin (\theta), \cos (\theta), 0), \\
& \mathbf{B}_{3}=(0,0,1) .
\end{aligned}
$$




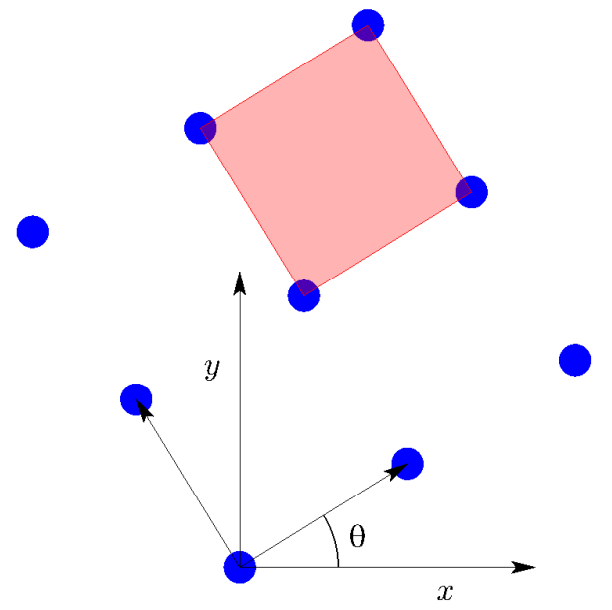

Figure 3.4: Orientation of a reproducible lattice for planar elongational flow.

For example, if $k=3$ and $N_{11}=2$, the magic angle would be $\theta \approx 31.72^{\circ}$ and the remapping time $\tau_{p}=0.9624 \dot{\epsilon}^{-1}$. These values have been used for the PEF simulations in this thesis. The orientation of the lattice vectors is shown in Figure 3.4

If the constructed lattice is deformed under PEF until $t=\tau_{p}$, the displaced lattice sites overlap with the initial lattice, as is shown in Figure 3.5. The initial and final lattice can both be described as a linear combination of the same set of basis vectors $\mathbf{B}_{i}$, with $i=1,2,3$. When this is the case, the lattice is reproducible. Todd and Daivis 29] and Baranyai and Cummings [31] independently applied this theory to perform NEMD simulations of a simple atomic fluid under PEF.

It is important, from a simulation point of view, to appreciate that the lattice does not strictly have to be remapped at time $t=\tau_{p}$. At each moment, the current lattice overlaps with the lattice that is exactly a time $\tau_{p}$ apart. This means that the reproducibility condition Eq. 3.45 can be rewritten into a more general form

$$
\mathbf{L}_{i}(t)=\mathbf{L}_{i}\left(t-\tau_{p}\right) \cdot \boldsymbol{\Lambda}\left(t-\left\lfloor\frac{t}{\tau_{p}}\right\rfloor\right), \quad \forall t,
$$

where $\lfloor\ldots\rfloor$ reduces its argument to the nearest smaller integer value. Due to this subtle point, the simulation time step $\Delta t$ does not need to be adjusted such that $\tau_{p}$ is an integer multiple of $\Delta t$. The deformation of the lattice vectors is described by a continuous function of time. Therefore, it is always possible to remap the lattice at any given time to a compatible (i.e., overlapping) lattice exactly $\tau_{p}$ earlier. 


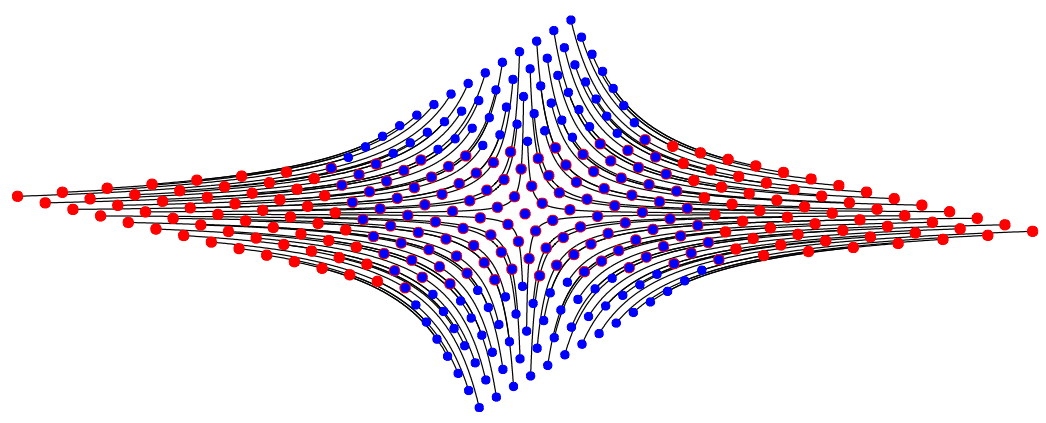

Figure 3.5: Typical reproducible lattice for PEF. The lattice is reproducible after a time $\tau_{p}$, allowing for indefinitely long simulations. Blue indicates the initial lattice, red is the lattice after time $\tau_{p}$. The lattice points collapse onto each other.

\subsection{Combined shear and elongational flows}

In the past, predominantly shear [27, 154, 155] flow and elongational flows [29, 143, 152, 153. 155 157. have been simulated due to their relative simplicity. Evans and Heyes [156] were the first, in 1990, to simulate a flow of combined elongation and shear. They were however limited by the lack of reproducible periodic boundary conditions, just like Heyes [152] was five years earlier for extensional flows. This problem has recently been overcome and a rigorous numerical study of planar mixed flow (PMF) has been performed by Hunt et al. [32. They simulated PMF, in which the velocity gradient is a linear combination of those for PCF (Eq. 3.30) and PEF (Eq. 3.37)

$$
\nabla \mathbf{u}=\left[\begin{array}{ccc}
\dot{\epsilon} & 0 & 0 \\
\dot{\gamma} & -\dot{\epsilon} & 0 \\
0 & 0 & 0
\end{array}\right]
$$

where $\dot{\epsilon}$ is the rate of deformation and $\dot{\gamma}$ the shear rate.

Apart from NEMD simulations of dense fluids under mixed flows, non-equilibrium Brownian dynamics (NEBD) simulations have been used to study molecules in a dilute solution under PMF. These studies have been performed without the reproducible periodic boundary conditions developed by Hunt et al. [32. Shaqfeh and coworkers used NEBD simulations to study the behavior of DNA [158 160] and linear polymers [161, 162 under a constant planar mixed flow. Very recently, Sing and Alexander-Katz 163. performed NEBD simulations to study the collapse of polymers under a range of mixed flows. Furthermore, Lee et al. [164] designed a microfluidic four-roll mill that can be used to study the behavior of fluids in a variety of flow types experimentally.

Substituting Eq. 3.55) in the SLLOD equations of motion (Eqs. 3.18) and (3.19), 


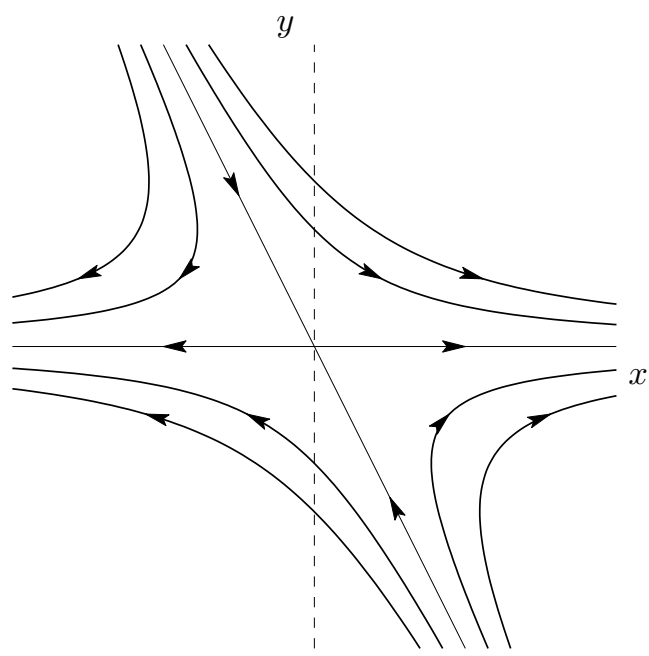

Figure 3.6: Typical streamlines for a planar mixed flow. The velocity gradient contains a combination of simple shear, with a gradient in the $y$-direction and planar elongation with extension in the $x$-direction and a contraction in $y$. This leads to a pure extension of the fluid in the $x$-direction and contraction along the direction of one of the eigenvectors of the velocity gradient. The strength of the shear and elongation are equal in this diagram. This ratio determines the angle between the directions of compression and extension.

the equations under which planar mixed flow evolves become:

$$
\begin{aligned}
\dot{\mathbf{r}}_{i} & =\frac{\mathbf{p}_{i}}{m_{i}}+\dot{\epsilon}\left(x_{i} \mathbf{e}_{\mathbf{x}}-y_{i} \mathbf{e}_{\mathbf{y}}\right)+\dot{\gamma} y_{i} \mathbf{e}_{\mathbf{x}} \\
\dot{\mathbf{p}}_{i} & =\mathbf{F}_{i}-\dot{\epsilon}\left(p_{x i} \mathbf{e}_{\mathbf{x}}-p_{y i} \mathbf{e}_{\mathbf{y}}\right)-\dot{\gamma} p_{y i} \mathbf{e}_{\mathbf{x}}-\zeta \mathbf{p}_{i}
\end{aligned}
$$

An example of streamlines for PMF is shown in Figure 3.6. The PMF velocity gradient has the same eigenvalues $\left(\lambda_{1}=\dot{\epsilon}, \lambda_{2}=0, \lambda_{3}=-\dot{\epsilon}\right)$ as the velocity gradient for planar elongational flow. However, the corresponding eigenvectors deviate. As opposed to PEF, the directions that correspond to shear-free contraction and expansion are not orthogonal. These directions correspond to eigenvectors of the velocity gradient, with an angle between the directions that is given by

$$
\phi=\cos ^{-1}\left(\frac{\dot{\gamma}}{\sqrt{\dot{\gamma}^{2}+4 \dot{\epsilon}^{2}}}\right) .
$$

For example, when $\dot{\gamma}=\dot{\epsilon}$, the smallest angle between both directions is $\phi=63.4^{\circ}$.

In order to find a compatible and reproducible lattice for PMF, we introduce a 
transformation matrix $\mathbf{Q}$ that consists of the eigenvectors of the velocity gradient

$$
\mathbf{Q}=\left[\begin{array}{ccc}
1 & 0 & 0 \\
\frac{\dot{\gamma}}{2 \dot{\epsilon}} & 1 & 0 \\
0 & 0 & 1
\end{array}\right]
$$

The evolution of the lattice for the Kraynik-Reinelt periodic boundary conditions was given by $\boldsymbol{\Lambda}=\exp (\nabla \mathrm{u} t)$. Similarly for mixed flow, if the velocity gradient can be diagonalized using $\mathbf{D}=\mathbf{Q}^{-1} \cdot \nabla \mathbf{u} \cdot \mathbf{Q}$, the evolution can be rewritten as

$$
\mathbf{\Lambda}(t)=\exp (\nabla \mathbf{u} t)=\exp \left(\mathbf{Q} \cdot \mathbf{D} \cdot \mathbf{Q}^{-1} t\right)=\mathbf{Q} \cdot \exp (\mathbf{D} t) \cdot \mathbf{Q}^{-1} .
$$

Substituting Eq. 3.60 into the condition for reproducibility of a lattice (Eq. (3.45) gives

$$
\begin{aligned}
\mathbf{L}_{i}^{\prime}\left(t=\tau_{p}\right) & =\mathbf{L}_{i}^{\prime}(0) \cdot \mathbf{\Lambda}\left(\tau_{p}\right) \\
& =\mathbf{L}_{i}(0) \cdot \mathbf{Q}^{-1} \cdot \mathbf{Q} \cdot \exp \left(\mathbf{D} \tau_{p}\right) \cdot \mathbf{Q}^{-1} \\
& =\mathbf{L}_{i}(0) \cdot \exp \left(\mathbf{D} \tau_{p}\right) \cdot \mathbf{Q}^{-1} \\
& =\left(N_{i 1} \mathbf{B}_{1}+N_{i 2} \mathbf{B}_{2}+N_{i 3} \mathbf{B}_{3}\right) \cdot \mathbf{Q}^{-1} \\
& =N_{i 1} \mathbf{B}_{1}^{\prime}+N_{i 2} \mathbf{B}_{2}^{\prime}+N_{i 3} \mathbf{B}_{3}^{\prime} \\
& =\mathbf{L}_{i}^{\prime}(0)
\end{aligned}
$$

which is similar to Eq. 3.45, with the only difference being the basis vectors that satisfy the reproducibility condition. From Eq. (3.64) we find that the basis vectors $\mathbf{B}_{i}^{\prime}$ are given by

$$
\begin{aligned}
& \mathbf{B}_{1}^{\prime}=\mathbf{B}_{1} \cdot \mathbf{Q}^{-1}=(\cos (\theta), \sin (\theta), 0) \cdot \mathbf{Q}^{-1}=\left(\cos (\theta)-\frac{\dot{\gamma}}{2 \dot{\epsilon}} \sin (\theta), \sin (\theta), 0\right), \\
& \mathbf{B}_{2}^{\prime}=\mathbf{B}_{2} \cdot \mathbf{Q}^{-1}=(-\sin (\theta), \cos (\theta), 0) \cdot \mathbf{Q}^{-1}=\left(-\sin (\theta)-\frac{\dot{\gamma}}{2 \dot{\epsilon}} \cos (\theta), \cos (\theta), 0\right), \\
& \mathbf{B}_{3}^{\prime}=\mathbf{B}_{3} \cdot \mathbf{Q}^{-1}=(0,0,1) \cdot \mathbf{Q}^{-1}=(0,0,1),
\end{aligned}
$$

As opposed to the basis vectors for planar elongational flow, the basis vectors of a lattice compatible with PMF are in general not orthogonal and not equal in length. Thus, the unit cell is a parallelogram. When the rate of elongation is much larger than the shear rate, the lattice vectors become almost orthogonal and equal in length. In the limiting case of zero shear rate, the lattice becomes equal to that for planar elongational flow. Figure 3.7 shows basis vectors for a mixed flow with $\dot{\gamma}=\dot{\epsilon}$ and the overlap of the initial and final lattice is shown in Figure 3.8 .

Remapping of the positions of the particles is automatically performed by the periodic boundary conditions after the lattice vectors are redefined. The mapping of the positions of the particles from the final to the initial cell is illustrated in Figure 3.9. Note that the relative distances between particles are not affected by the mapping, 


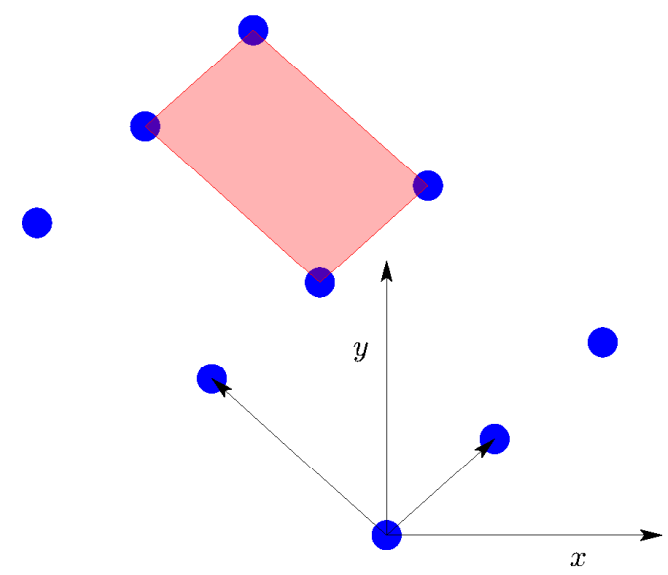

Figure 3.7: Initial cell for $\operatorname{PMF}(\dot{\gamma}=\dot{\epsilon})$.

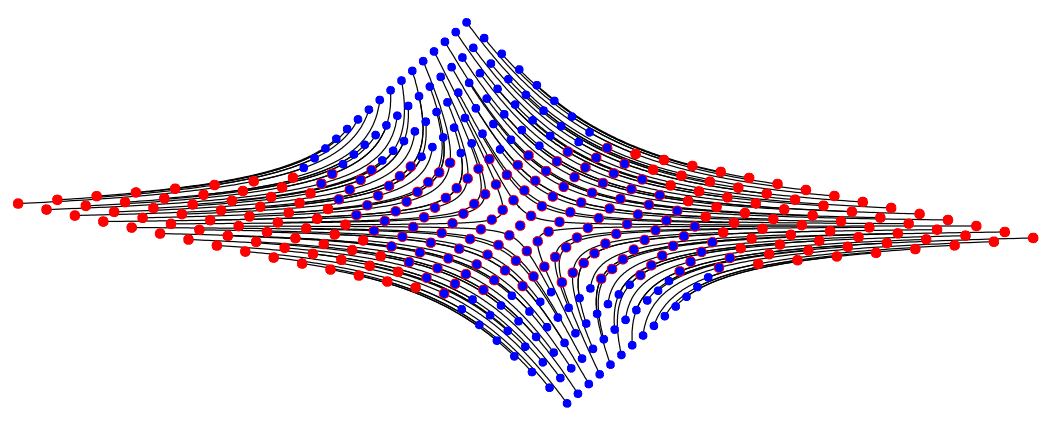

Figure 3.8: Typical reproducible lattice for PMF $(\dot{\gamma}=\dot{\epsilon})$. The lattice is reproducible after a time $\tau_{p}$, allowing for indefinitely long simulations. Blue indicates the initial lattice, red is the lattice after time $\tau_{p}$. 


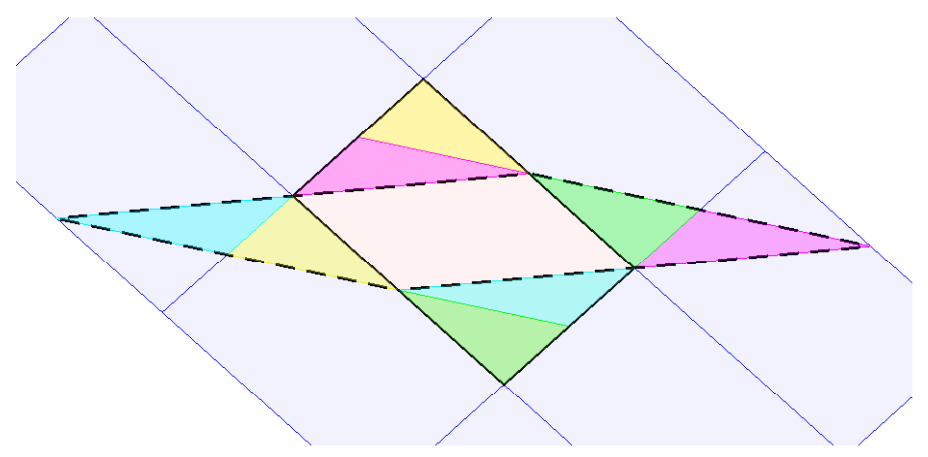

Figure 3.9: Atomic PMF simulation $(\dot{\gamma}=\dot{\epsilon})$. The solid black line indicates the initial cell and the dotted line is the deformed cell. When the simulation cell is remapped, the particles are mapped back onto the initial cell according to the colors of the areas, i.e., particles in the red area in the deformed cell are mapped to the red area in the undeformed cell, and similarly for the other colors.

neither are the velocities affected, since the SLLOD equations of motion only couple the peculiar velocities explicitly to the particles.

When the cell is deformed, the minimum lattice spacing must always be larger than twice the cutoff radius of the interaction potential. The three distances that should be checked for (all in the plane of deformation) are given by

$$
\begin{aligned}
& \mathbf{r}_{1}(0)=L_{1} \mathbf{B}_{1}^{\prime} \\
& \mathbf{r}_{2}(0)=L_{2} \mathbf{B}_{2}^{\prime}, \\
& \mathbf{r}_{3}(0)=L_{1} \mathbf{B}_{1}^{\prime}+L_{2} \mathbf{B}_{2}^{\prime},
\end{aligned}
$$

with $L_{1}$ and $L_{2}$ the length of the simulation cell in the directions of the basis vectors $\mathbf{B}_{1}^{\prime}$ and $\mathbf{B}_{2}^{\prime}$, respectively. These vectors are shown in Figure 3.10. Note that all the final cell vectors are simply a linear combination of the initial cell vectors, whereas the cell vectors of the half-way shape $\left(t=\tau_{p} / 2\right)$ is not simply a linear combination of the initial vectors. Hence, the cell is not reproducible at this point in time. The minimum length in the directions of the cell vectors is derived from the trajectories of the cell vectors $\mathbf{r}(t)=(x(t), y(t))$ (shown as blue dashed lines in the figure)

$$
\begin{aligned}
x(t) & =\frac{\dot{\gamma}}{\dot{\epsilon}} y(0) \sinh (\dot{\epsilon} t)+x(0) \exp (\dot{\epsilon} t) \\
y(t) & =y(0) \exp (-\dot{\epsilon} t)
\end{aligned}
$$

The length of each of the vectors $\mathbf{r}_{1}(t), \mathbf{r}_{2}(t)$ and $\mathbf{r}_{3}(t)$ reaches a minimum when

$$
\frac{d}{d t}(\mathbf{r}(t) \cdot \mathbf{r}(t))=2(x(t) \dot{x}(t)+y(t) \dot{y}(t))=0 .
$$




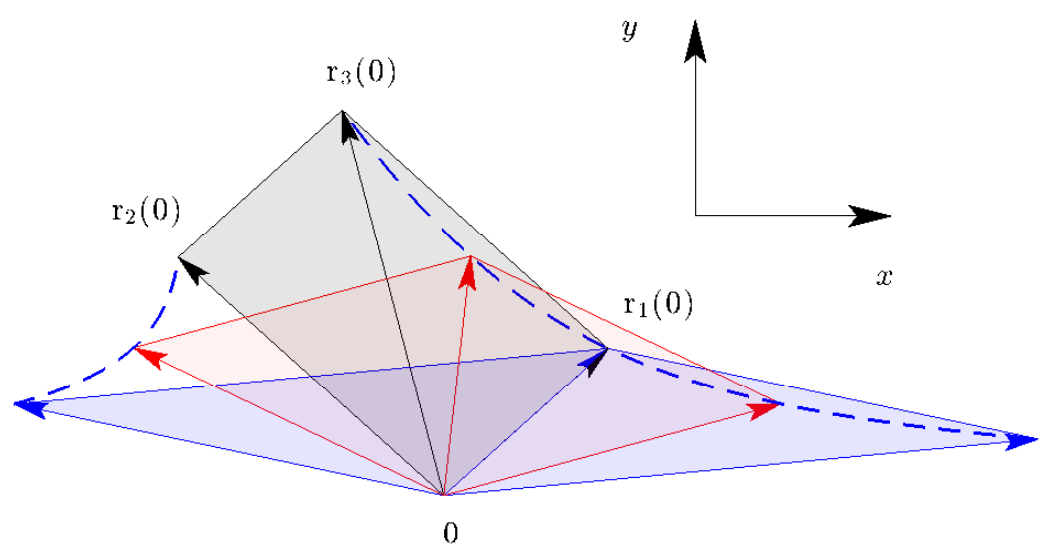

Figure 3.10: Deformation of a simulation cell under PMF, with $\dot{\gamma}=\dot{\epsilon}$. The initial cell $(t=0)$ is shown in black, half way $\left(t=\tau_{p} / 2\right)$ in red and the final cell $\left(t=\tau_{p}\right)$ in blue.

The time at which the minimum length is reached, for each direction, can be expressed in terms of the field and the initial vector coordinates. By substituting Eqs. 3.71) and (3.72) into Eq. (3.73), the time $t_{m}$ at which a vector $\mathbf{r}(t)$ reaches a minimum length can be calculated to be

$$
t_{m}=\frac{1}{4 \dot{\epsilon}} \ln \left(\frac{\dot{\gamma}^{2} y(0)^{2}+4 \dot{\epsilon}^{2} y(0)^{2}}{(\dot{\gamma} y(0)+2 \dot{\epsilon} x(0))^{2}}\right) .
$$

Subsequently, this time corresponds to a vector length given by

$$
D_{m}=\sqrt{x\left(t_{m}\right)^{2}+y\left(t_{m}\right)^{2}} .
$$

The size of the simulation cell should be chosen such that $D_{m} \geq 2 r_{c}$.

Applying these reproducible periodic boundary conditions to a NEMD simulation allows for indefinitely long simulations, such that sufficiently long time-averaging of useful properties can be performed. Figure 3.11 shows snapshots of a simulation of an atomic fluid under PMF $(\dot{\gamma}=\dot{\epsilon})$. The snapshots span exactly one period $\tau_{p}$ of the periodic boundaries. 

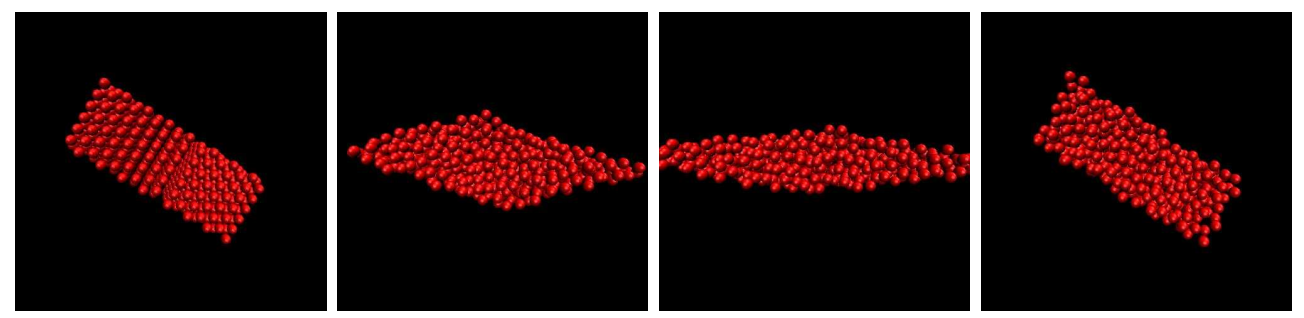

Figure 3.11: Simulation of a WCA fluid under PMF. 


\section{4}

\section{Statistical mechanics}

Statistical mechanics and physics connect the microscopic atomistic world, as modeled via MD, to macroscopic quantities and balance equations of fluid dynamics and thermodynamics. Equilibrium statistical mechanics is a well-established field, whereas a statistical mechanics treatment of non-equilibrium problems is often much more difficult. Two non-equilibrium statistical mechanics approaches can be distinguished. The first one is based on computing the non-equilibrium distribution function that describes the probability for each unique microstate to occur. This approach is not practically feasible in most cases. The second approach uses correlation functions to describe quantities out of equilibrium. While a detailed theoretical treatment is beyond the scope of this work, some of the tools used in non-equilibrium statistical mechanics are discussed in this chapter.

The outline of this chapter is as follows: In Section 4.1 the general phase space description of a multi-body system is discussed. Statistical ensembles are introduced in Section 4.2. Next, in Section 4.3, correlation functions are introduced. The calculation of linear transport coefficients is discussed in Section 4.4 and Section 4.5 presents a method to calculate the nonlinear transport properties. Finally, in Section 4.6, the pair distribution function is introduced.

\subsection{General phase space description}

A phase space vector $\boldsymbol{\Gamma}=\left(\mathbf{r}_{1}, \ldots, \mathbf{r}_{N}, \mathbf{p}_{1}, \ldots, \mathbf{p}_{N}\right)$ denotes a single microscopic state (or 'microstate') in a $6 N$-dimensional phase space, that contains all possible combinations of positions $\mathbf{r}=(x, y, z)$ and momenta $\mathbf{p}=\left(p_{x}, p_{y}, p_{z}\right)$ of all particles in a three-dimensional $N$-body system. By sampling many microstates that span the part of phase space that satisfies the constraints on the system, a picture is formed of the probability distribution of microstates. If the sample is large enough, the number 
of systems that correspond to a certain microstate can be considered to change continuously if we move through phase space. Consequently, the probability of a given microstate to occur at a time $t$ can be described by a phase space distribution function $f(\boldsymbol{\Gamma}, t)$. Similarly, the distribution function predicts the fraction of systems $\delta \Omega$ that are present in an infinitesimally small phase space volume element $\delta \boldsymbol{\Gamma}$ at time $t$

$$
\delta \Omega=f(\boldsymbol{\Gamma}, t) \delta \boldsymbol{\Gamma},
$$

which can be integrated over the entire phase space to obtain $\int f(\boldsymbol{\Gamma}, t) d \boldsymbol{\Gamma}=1$. The distribution function has to be smooth in time and in terms of the phase space variables (i.e., the function has continuous partial derivatives with respect to all phase space variables).

If the distribution function would be exactly known, the instantaneous ensembleaveraged properties of the system could be calculated. This is often not the case, and statistical sampling is required to calculate properties. A system corresponds to a single microstate at every moment in time and from this microstate various fluid properties can be calculated (e.g., the instantaneous pressure tensor). As the simulation advances in time, the system moves through phase space, spending more time on average in regions with a higher probability and no time in regions with zero probability. Using this feature, the probability density can be calculated by sampling points in phase space.

When a system is in equilibrium, or in a non-equilibrium steady state, an average phase variable $\langle B\rangle$ could be calculated by time-averaging a walk through phase space. In order to accumulate enough information to obtain a small uncertainty, very long time-averages are generally required, which is often not practically feasible.

Instead, information can be accumulated by averaging over ensembles. If the system is ergodic, then time averaging and ensemble averaging are the same in the statistical limit. Note that both types of averaging can also be combined. The standard error of an averaged quantity is inversely proportional to the square root of the amount of information used. Figure 4.1 shows the averaged shear stress for a fluid with density $\rho=0.84$ and temperature $T=1.0$. The fluid is sheared at a rate $\dot{\gamma}=0.01$, which is an order of magnitude smaller than the typical shear rates in NEMD simulations. Because of smaller signal-to-noise ratio, more averaging is required to obtain a small relative uncertainty. The shear stress shown in the figure is calculated from a total of $N_{S}=50$ simulations, each containing $N=512$ particles, averaged over a time period $N_{T}=20,000$ in reduced units. The data plotted in blue correspond to a situation in which the full time average is used, but only a portion of the total number of simulations. The data plotted in red is averaged over all 50 simulations but the period over which the time averaging is performed varies. The $100 \%$ on the $x$-axis corresponds to an average over all simulations and the full period of time. The figures shows how the 


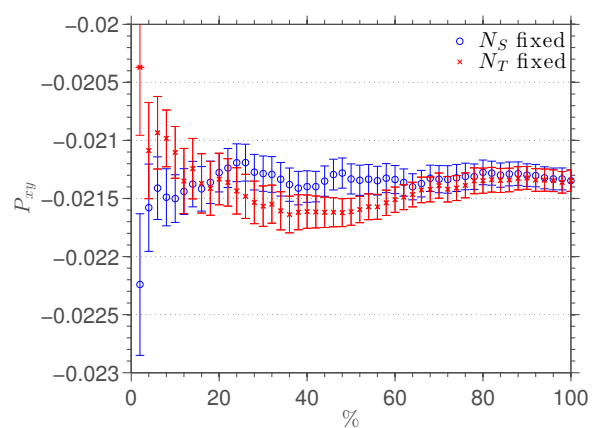

(a)

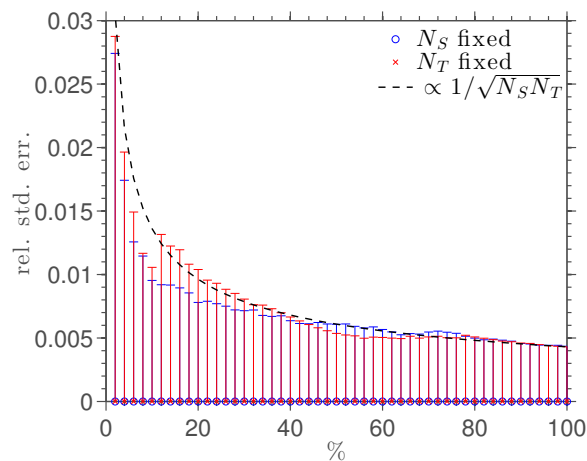

(b)

Figure 4.1: Convergence of the average shear stress value and corresponding standard error as a function of the amount of information used.

shear stress (Figure 4.1(a) and the normalized standard error (Figure 4.1(b) converge as a larger portion of the information is used for the averaging. The standard error decays as $e \propto 1 / \sqrt{N_{S} \times N_{T}}$. The trend in Figure 4.1(b) is scaled by the error at $100 \%$, where the standard error is approximately $0.4 \%$ of the calculated shear stress value.

If a system is said to be ergodic, it will eventually reach every microstate that has a non-zero probability. In ergodic systems, the time-averaging of any phase variable $B(\boldsymbol{\Gamma})$ can be replaced by an average over phase-space

$$
\int f(\boldsymbol{\Gamma}) B(\boldsymbol{\Gamma}) d \boldsymbol{\Gamma}=\lim _{t \rightarrow \infty} \frac{1}{t} \int_{0}^{t} B\left(t^{\prime}\right) d t^{\prime} .
$$

A simple way to think of ergodicity is to imagine a person walking around on an open field. That person would cover the whole area (phase space) if he would walk around indefinitely in a random manner. Similarly, if he would be dropped many times, at random locations in the field, he would also cover the whole area. His location (microstate) in time depends on where he has been in the near past, but he can and will eventually reach every point, regardless of this initial location. If the person is dropped in the same field, but now with high fences distributed over the area, he could not reach any possible location, depending on his initial location. Therefore, his location in time is dependent on the initial location. On the other hand, if the person is dropped many times at random locations in that same field, he would still cover the whole area at some point, despite the fences. The system has not become non-ergodic.

Computer simulations of multi-body and multidimensional systems are much more difficult to imagine as the phase space is a $6 N$-dimensional object. The ergodicity of dynamical systems are related to their chaotic properties. Lyapunov exponents 
describe the rate of separation between nearby phase-space trajectories and serve as a measure for chaos [165-167]. If chaos is present, the current state quickly looses it dependence on previous states, such as was the case for the person in the open field.

\subsection{Statistical ensembles}

The distribution of probabilities depends on the properties of the system. The simplest case is a system in which the number of particles $N$, volume $V$ and energy $E$ are conserved. These state variables are automatically conserved in a periodic system with a fluid in equilibrium where the particles follow Newtonian dynamics. A collection of microstates that satisfy the given conditions on a system form a so-called ensemble. Systems in which the number of particles, volume and energy are conserved are said to be in a microcanonical $(N V E)$ ensemble. In a Hamiltonian system, each microstate that satisfies these conditions has an equal probability of occurring. Consequently, microscopic states that do not satisfy the conditions of the ensemble have a zero probability. Hence, the probability distribution of microstates is given by

$$
f_{E}(\boldsymbol{\Gamma})=\frac{\delta\left(\mathcal{H}_{0}-E\right)}{\int \delta\left(\mathcal{H}_{0}-E\right) d \boldsymbol{\Gamma}},
$$

where $\mathcal{H}_{0}$ is the Hamiltonian of the system

$$
\mathcal{H}_{0}=\sum_{i=1}^{N} \frac{\mathbf{p}_{i} \cdot \mathbf{p}_{i}}{2 m_{i}}+U\left(\mathbf{r}_{1}, \ldots, \mathbf{r}_{N}\right)
$$

The distribution function is normalized such that it satisfies $\int f_{E}(\boldsymbol{\Gamma}) d \boldsymbol{\Gamma}=1$. The normalization factor in the denominator of a distribution function is called the 'partition function'.

Boltzmann derived a microscopic expression for the entropy in an microcanonical system

$$
S=\mathrm{k}_{\mathrm{B}} \int f_{E} \ln f_{E} d \boldsymbol{\Gamma}=\mathrm{k}_{\mathrm{B}} \ln \Omega(N, V, E)
$$

where $\Omega$ is the number of configurations compatible with a given state. The microcanonical ensemble corresponds to an isolated system, in which no exchange of mass, work or heat takes place with the outside world. This ensemble is often not very practical for the study of real systems, due to the fact that most real systems exchange at least mass, work or heat with their environments.

A canonical ensemble is much more often used in MD than the microcanonical ensemble. In this ensemble, the number of particles $N$, volume $V$ and temperature $T$ are conserved. This corresponds to a closed system, where energy is exchanged 
with the environment. Figure 4.2 shows a schematic representation of a microcanonical (isolated) and a canonical (closed) system. The last situation (an open system) shown in the figure allows for the exchange of mass, work and heat. A fully open system rarely appears in MD simulations. The grand canonical ensemble allows for a varying number of particles, while fixing the chemical potential, fluid temperature and system volume. This ensemble is more common in Monte Carlo (MC) simulations. Furthermore, hybrid schemes exist that combine multiple ensembles or couple systems to each other that have the same ensemble. For example, the dual control volume grand canonical molecular dynamics ensemble (DCV-GCMD) [168] connects two chemical potential control volumes in order to produce a steady state chemical potential gradient. The phase-space distribution function is a conserved property in

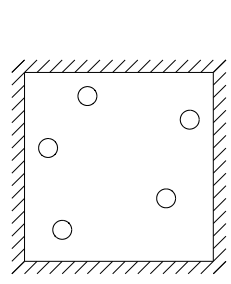

Is olated system

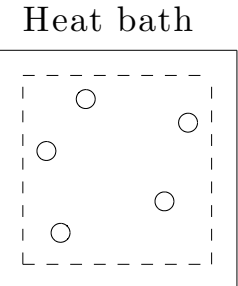

Closed system

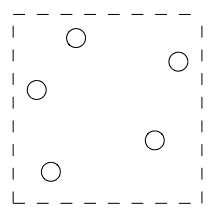

Open

system

Figure 4.2: Isolated (microcanonical), closed (canonical) and open system.

systems where the equations of motion can be derived from a Hamiltonian. Hamilton's equations are appropriate for describing isolated or certain closed systems [119]. The mass and heat transfers in open systems cannot be described by Hamiltonian mechanics.

The phase-space distribution function in non-Hamiltonian systems is not preserved and has fractal properties. Furthermore, the volume of phase space can collapse as the systems advances. The adiabatic incompressibility of phase space (AIГ) is a condition that determines if the volume of phase space is preserved when the system advances 28. This condition is satisfied if the phase space compression factor $\Lambda \equiv \partial \dot{\boldsymbol{\Gamma}} / \partial \boldsymbol{\Gamma}$ is identical to zero, which is always the case for Hamiltonian equations of motion (see Eq. (2.9)), but not for all non-Hamiltonian systems. Many non-equilibrium systems are non-Hamiltonian, which can make a statistical mechanics treatment exceptionally challenging [169].

The probability of a certain microstate $\boldsymbol{\Gamma}$ to occur in a canonical ensemble is given by the following distribution function

$$
f_{c}(\boldsymbol{\Gamma})=\frac{\mathrm{e}^{-\beta \mathcal{H}_{0}}}{\int \mathrm{e}^{-\beta \mathcal{H}_{0}} d \boldsymbol{\Gamma}},
$$


where $\beta=\left(\mathrm{k}_{\mathrm{B}} T\right)^{-1}$.

In an MD simulation, a fluid in equilibrium is by default in the microcanonical ensemble if no thermostat is coupled to the fluid. If a Nosé-Hoover thermostat is coupled to a fluid, the probability distribution of microstates will satisfy the canonical ensemble in general, as discussed in Section 2.5.2.

A system is referred to as adiabatic when the temperature is not explicitly controlled by a thermostat. While heat is not directly exchanged with the environment in adiabatic systems, energy is added to the system if an external force is present that drives the fluid away from equilibrium. In this case, the temperature in the fluid increases due to viscous heating. Adiabatic equations of motion can be useful for theoretical analysis, but are rarely applied to atomistic simulations. This is different, for example, in simulations of dissipative granular materials, where the coefficient of restitution is smaller than unity. An example of a gravity-driven granular flow down an inclined chute can be found in Ref. [147].

\subsection{Time-correlation functions}

It is often useful to follow the ensemble average of a product of two quantities as time progresses. This ensemble average is called a correlation function $C_{A B}(t)$ and describes the correlation between quantities $A$ and $B$, as a function of their separation in time $t$. The decay of a correlation can say a lot about the properties of a dynamical system.

We can use the phase space distribution function presented in Section 4.1 to derive an expression for the time-correlation function

$$
C_{A B}(t)=\langle A(t) B(0)\rangle=\int f(\boldsymbol{\Gamma}) B(\boldsymbol{\Gamma}) \mathrm{e}^{i L t} A(\boldsymbol{\Gamma}) d \boldsymbol{\Gamma},
$$

where $\mathrm{e}^{i L t}$ is the propagator of the observable $A$ and the brackets $\langle\ldots\rangle$ denote an ensemble average [28. The propagator is a mathematical operator that uses the equations of motion to calculate the evolution of a phase variable from its initial value. Despite its theoretical relevance, Eq. 4.6. is not practical in MD since it requires knowledge of the phase space distribution function and a sampling of many microstates. Since the correlations in equilibrium and non-equilibrium steady state are invariant to a shift in time origin, the correlation function can be calculated by averaging over distinct trajectories at different moments in time. In molecular dynamics simulations, time is discretized and the correlation function can be calculated from a single steady-state simulation as

$$
C_{A B}(t)=\frac{1}{N_{t}} \sum_{i=1}^{N_{t}} A\left(t_{i}+t\right) B\left(t_{i}\right), \quad t_{i}=0, \tau, 2 \tau, \ldots,\left(N_{t}-1\right) \tau
$$


where $t_{i}$ is the starting time of a trajectory, $\tau$ the time between the starting points of two consecutive trajectories and $N_{t}$ number of trajectories to average over. The time difference $\tau$ between the beginning of two consecutive trajectories should be chosen such that the initial states of consecutive trajectories are uncorrelated to each other. Therefore, it would be safe, although conservative, to choose $\tau \geq \max (t)$.

If phase space mixing occurs, correlation functions decay from $\langle A(0) B(0)\rangle$ at time $t=0$, to $\langle A\rangle\langle B\rangle$ in the limit of $t \rightarrow \infty$ [170]. Calculating quantities that are expressed in terms of a correlation function can be computationally expensive if the correlation function decays slowly. An example is the zero-shear first normal stress coefficient that will be treated in Chapter 5 .

In the calculation of an equilibrium correlation function, the origin of the time is arbitrary and the ensemble distribution function is invariant to time. Hence the correlation can be shifted in time [171]. Consequently, the following property is satisfied

$$
C_{A B}(t)=\langle A(t) B(0)\rangle=\langle A(0) B(-t)\rangle=C_{A B}(-t) .
$$

This property makes the correlation function an even function of time, such that a Taylor series expansion of a real-valued correlation function around the origin $t=0$ is given by 63 .

$$
C_{A B}(t)=\sum_{n=0}^{\infty} \frac{t^{2 n}}{(2 n) !}\left\langle A^{(2 n)}(0) B(0)\right\rangle,
$$

where the brackets in the superscripts denote derivatives with respect to time. This expression can be rewritten, using the following property

$$
\begin{aligned}
\langle\ddot{A}(t) B(0)\rangle & =\lim _{t \rightarrow \infty} \int_{0}^{t} \ddot{A}(t-s) B(s) d s \\
& =-\lim _{t \rightarrow \infty} \int_{0}^{t} \dot{A}(t-s) \dot{B}(s) d s=-\langle\dot{A}(t) \dot{B}(0)\rangle .
\end{aligned}
$$

Similarly, this can be applied to higher order derivatives, resulting in

$$
\begin{aligned}
C_{A B}(t) & =\sum_{n=0}^{\infty}(-1)^{n} \frac{t^{2 n}}{(2 n) !}\left\langle A^{(n)}(0) B^{(n)}(0)\right\rangle \\
& =\langle A(0) B(0)\rangle-\frac{t^{2}}{2 !}\left\langle A^{(1)}(0) B^{(1)}(0)\right\rangle+\frac{t^{4}}{4 !}\left\langle A^{(2)}(0) B^{(2)}(0)\right\rangle+\mathcal{O}\left(t^{6}\right) .
\end{aligned}
$$

The functional form of this expansion is used to find a suitable fitting function for the short time decay of a correlation function in Chapter 5 .

The correlation of a quantity with itself is called an autocorrelation function $C_{A A}(t)$. In this work, we are mostly interested in autocorrelation functions for their application in the calculation of transport coefficients. The velocity autocorrelation function 


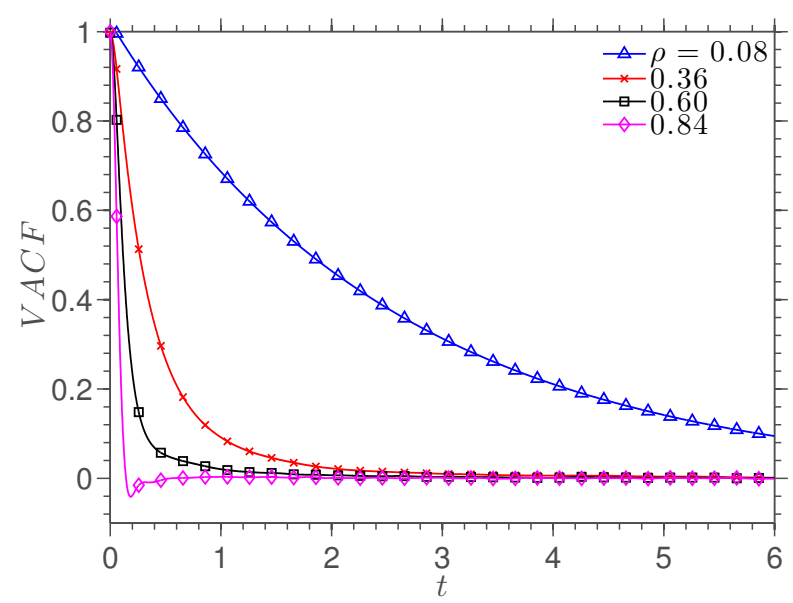

Figure 4.3: Velocity autocorrelation function for a gas and a liquid. The temperatures of the gas and liquid are $T=1.0$ and the densities are $\rho=0.08,0.36,0.60$ and 0.84 .

(VACF) and the stress autocorrelation function (SACF) are the most studied autocorrelation functions in molecular dynamics, since they are related to self-diffusion and shear viscosity, respectively. These correlation functions can both be difficult to calculate accurately in simulations. The VACF is known to have a hydrodynamic tail 172 175]. For a hard-sphere fluid, this tail has been shown to decay with time as $t^{-d / 2}$, where $d$ is the dimensionality of the system [172]. Later studies have demonstrated that an algebraic tail is similarly observed for soft spheres and at a variety of state points 173 .

The VACF is known to have a long-time hydrodynamic tail [172], which does not satisfy the exponential decay that is predicted by the Boltzmann and Enskog theories. For a dilute hard-sphere fluid, it can be assumed that collisions are independent of each other. In that case, the autocorrelation function would decay exponentially. In practice, fluids have a memory and the correlation function decays slower than exponential. An example of the VACF for a WCA fluid at several densities is given in Figure 4.3. The correlation function of the dilute fluid $(\rho=0.08)$ decays approximately exponentially, whereas the decay of correlation functions that correspond to denser liquids show a more complicated behavior, including a negative correlation in the most dense case $(\rho=0.84)$ shown in the figure. This negative value is caused by the fact that the direction of the movement of atoms, and thus their velocity vectors, change after a collision.

The SACF's found in molecular dynamics simulations of hard-sphere fluids agree with the Enskog prediction at low density, where the kinetic part of the pressure tensor 
is dominant and the configurational part is negligible. Generalized kinetic theory [176] and an early mode coupling theory (MCT) calculation [177] both predict that the tail of the SACF should decay as $t^{-3 / 2}$ due to hydrodynamic effects that only contributes to the kinetic part of the stress autocorrelation function. However, the predicted hydrodynamic long time tail in the stress autocorrelation function has only ever been (tentatively) observed by isolating the kinetic part of the stress autocorrelation function [178. The total stress autocorrelation function also includes a configurational part and a cross term. Both of these exhibit long time tails of amplitude much greater than that predicted by the conventional MCT [178. Extended MCT [179 181] provides a much better prediction of the SACF behavior at moderate to high density. It predicts that the configurational and cross terms of the SACF decay as approximately $t^{-3 / 2}$ at intermediate times, but more rapidly at very long times. The amplitude of the intermediate time contribution is enhanced compared to the hydrodynamic tail predicted by kinetic theory and simple MCT, and its amplitude grows with increasing density. The existence of this part of the stress relaxation function, known as the 'molasses tail' to distinguish it from the hydrodynamic long time tail, is linked to structural relaxation [182, 183] and the glass transition [184]. Although the extended mode coupling theory provides predictions of the stress relaxation function that agree with the results of molecular dynamics simulations of hard-sphere fluids, they do not result in a definite functional form that can easily be used to describe the whole relaxation function.

Equilibrium and non-equilibrium SACF's are analyzed in detail in Chapters 5 and 6. respectively.

\subsection{Calculation of Navier-Stokes transport coefficients}

The importance of transport coefficients, to close the system of conservation equations, is illustrated in Section 1.3 . These transport coefficients can be scalars or tensors of rank 2 or 4 , however only scalar transport coefficients are considered here. Nonequilibrium transport coefficients can be measured directly from NEMD simulations by taking the appropriate ratio between a flux $J$ and force $F^{E}$. The equilibrium coefficient can then be approximated by extrapolating the non-equilibrium result towards the zero force-field limit

$$
L=\lim _{F^{E} \rightarrow 0} \lim _{t \rightarrow \infty} \frac{\langle J\rangle_{t}}{F^{E}},
$$

where the notation $\langle\ldots\rangle_{t}$ denotes time averaging for a system in steady state. If the system is ergodic, the time average may be replaced with an ensemble average. Very long time averages are generally needed near equilibrium $\left(F^{E} \approx 0\right)$ in order to obtain a high signal-to-noise ratio. Furthermore, if results need to be extrapolated, multiple non-equilibrium simulations would be required with different forces. Finally, 
the thermostatting mechanism can influence the measurements of nonlinear transport properties, which then affects an extrapolation to equilibrium. Normal stress differences (and their coefficients) are a known example of such a situation [185.

Equilibrium statistical mechanics provides a way to calculate equilibrium transport coefficients by evaluating a time-integral over the appropriate equilibrium autocorrelation function

$$
L \propto \int_{0}^{\infty}\langle J(0) J(t)\rangle_{0} d t
$$

where the notation $\langle\ldots\rangle_{0}$ denotes a correlation function calculated at equilibrium $\left(F^{E}=0\right)$. The fluxes in a fluid at equilibrium only exhibit thermal fluctuations, such that they are zero on average $\langle J\rangle_{t}=0$. Hence, Eq. 4.14 relates the thermal fluctuations in a field to the macroscopic transport properties of a fluid. This approach is based on the Onsager regression hypothesis [140, 141], that states that the fluctuations of a flux in equilibrium are governed by the same transport coefficient as relaxation of that same flux out of equilibrium. For this insight, Lars Onsager was awarded the Nobel Prize in chemistry in 1968.

The Green-Kubo relations calculate a Navier-Stokes transport coefficient from the thermal fluctuation for a system in equilibrium. Based on Eq. 44.14, Green [186] and Kubo [187] developed expressions for the shear viscosity, bulk viscosity, self-diffusion and thermal conductivity. Only the shear viscosity is explicitly treated here, for its relevance to the work presented in this thesis. The non-equilibrium viscosity is calculated from the following relation: $\eta(\dot{\gamma})=-P_{x y} / \dot{\gamma}$, where $\dot{\gamma}$ is the shear rate. We can either obtain the Newtonian shear viscosity $\eta_{0}$ from extrapolating non-equilibrium simulation results to the zero-shear limit, or we can apply the Green-Kubo relation for shear viscosity

$$
\eta_{0}=\frac{V}{\mathrm{k}_{\mathrm{B}} T} \int_{0}^{\infty}\left\langle P_{x y}(t) P_{x y}(0)\right\rangle_{0} d t,
$$

where $P_{x y}$ is the shear stress, $V$ the volume of the system and $T$ the temperature.

While the Green-Kubo relations are calculated in equilibrium, they also predict non-equilibrium properties as long as these are linear in terms of the external force. For example, when the shear stress is a linear function of the shear rate and there is no residual shear stress at zero shear rate, the ratio between shear stress and shear rate is a constant, equal to the Newtonian viscosity. If the ratio between the shear stress and shear rate is not constant, the fluid is said to behave in a non-Newtonian manner. The validity of the Green-Kubo result away from equilibrium is illustrated in Figure 4.4. The figure shows the shear stress against the shear rate for a simple WCA fluid at a density $\rho=0.8442$ and temperature $T=0.722$. The NEMD results are in good agreement with the Newtonian approximation for shear rates up to $\dot{\gamma} \approx 0.1$. For larger shear rates, shear thinning occurs and the stress becomes non-linearly related 
to the driving field.

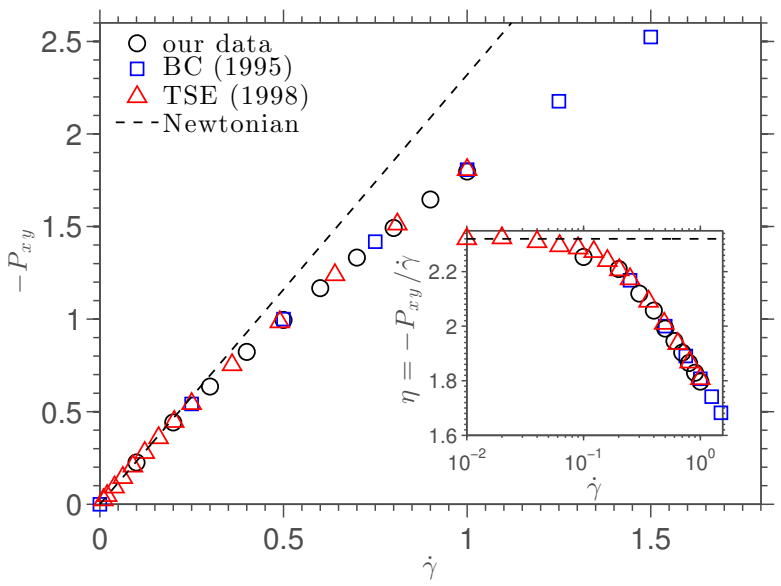

Figure 4.4: Shear stress as a function of shear rate. The dots give the NEMD result, whereas the dashed line is the Newtonian approximation. The label 'BC' corresponds to data presented by Baranyai and Cummings 31] and the label 'TSE' to data from Travis et al. [188. The slope of the dashed line corresponds to the Newtonian viscosity $\eta_{0}=2.32$. The inset shows the shear viscosity, calculated as the shear stresses divided by their respective shear rates.

\subsection{Transient-time correlation function}

The Green-Kubo relations express the linear transport coefficients in terms of integrals over the appropriate equilibrium correlation functions. These relations are based on the assumption that the response of a fluid is a linear function of the applied external field. This is often not true, in which case a nonlinear method is required to predict non-equilibrium flow properties.

Eq. (4.13) showed the expression for a linear transport coefficient in the limiting case of a zero force field. In case a steady-state system is far enough away from equilibrium, such that the linear approximation breaks down, Eq. 4.13 should be modified to

$$
L\left(F^{E}\right)=\lim _{t \rightarrow \infty} \frac{\langle J\rangle_{t}}{F^{E}}
$$

which is valid for an arbitrary external field. It is also possible to extend linear-response theory in the nonlinear regime using nonlinear-response theory. The formalism of the transient-time correlation function (TTCF) method [28, 176, 189] turns out to be of more practical use for both time independent and time dependent driving fields. 
For an arbitrary phase-space variable $B(t)$ and external force $F^{E}$, the response is given by

$$
\langle B(t)\rangle=\langle B(0)\rangle-\beta F^{E} \int_{0}^{t}\langle B(s) J(0)\rangle d s,
$$

where $J(0)$ is the dissipative flux at time $t=0$. Even though the Green-Kubo expression looks quite similar to that of TTCF, the time-correlation functions in this formulation are evaluated away from equilibrium after a constant external driving field has been activated. While this expression is derived from the Heisenberg representation of a phase-space average (as will be shown in Section 6.1), knowledge of the phase-space distribution function is not required.

In order to apply this expression, a set of equations of motion is needed in which the driving field is coupled to the fluid via their equations of motion. A well-known example of such equations are the SLLOD equations of motion, which will be discussed in Chapter 3. Only a handful of studies have appeared in which TTCF has been applied, most of them to atomic shear flow. Some recent work has focussed on applying TTCF to confined fluids [190, 191]. In Chapter6, the TTCF formulation is derived and the method is used for the study of three flow problems.

\subsection{Pair distribution functions}

The microstructure of a fluid can be studied with the pair correlation function $g\left(\mathbf{r}_{i j}, \nabla \mathbf{u}\right)$, where $\mathbf{r}_{i j}=\mathbf{r}_{i}-\mathbf{r}_{j}$ is a vector between the centers of two particles, or the structure factor $S(\mathbf{k}, \nabla \mathbf{u})$, where $\mathbf{k}$ denotes the wave vector [46]. These functions give information about the structural arrangement (i.e., the average relative distances and orientations) of atoms in a fluid or solid. In this section, pair distribution functions for equilibrium and non-equilibrium systems are briefly introduced.

The distribution of interactions between atoms in a homogeneous atomic fluid in equilibrium has no preferred direction 11 Hence, one can simplify the distribution function to a function of distance only. This special case is known as the radial distribution function (RDF), denoted by $g(r)$. The RDF can be calculated by

$$
g(r)=\frac{V}{8 \pi r^{2} N^{2}}\left\langle\sum_{i=1}^{N} \sum_{j \neq i} \delta\left(r-r_{i j}\right)\right\rangle,
$$

where $r_{i j}=\left|\mathbf{r}_{i}-\mathbf{r}_{j}\right|, N$ is the number of particles and $V$ the volume of the system. The radial distribution function for simple fluids can be used to calculate equilibrium fluid properties. For example, Zwanzig and Mountain [192 expressed the infinite frequency shear and bulk moduli in terms of the radial distribution function.

\footnotetext{
${ }^{1}$ Unless one considers liquid crystals, where a regular structure is formed in the configuration of the atoms.
} 


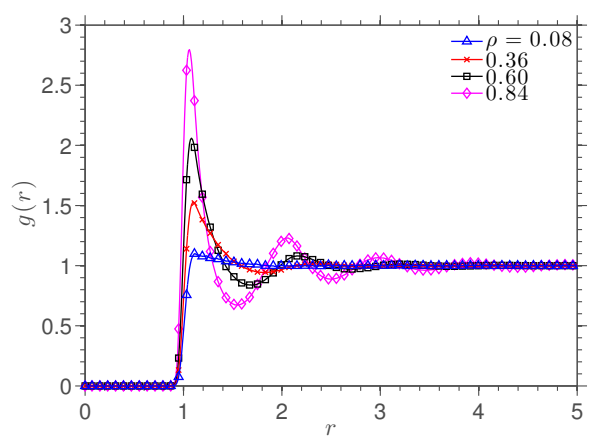

(a)

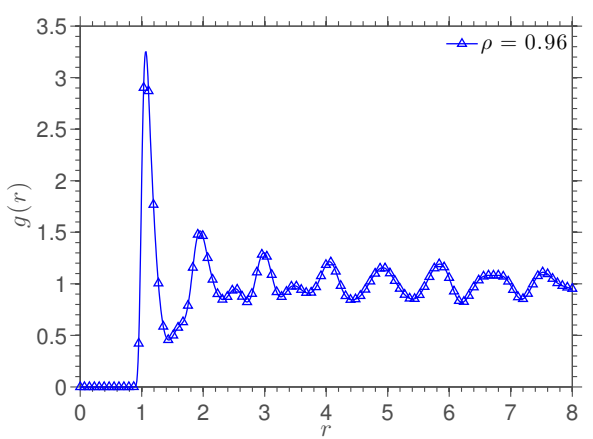

(b)

Figure 4.5: Radial distribution functions for WCA fluids at temperature $T=1.0$ and various densities. Distribution functions that correspond to the gas and liquid phase are shown in (a), whereas (b) shows the distribution function of an amorphous solid.

The RDF can be seen as the local density of particles. The function is commonly normalized by the average fluid density, such that it converges to unity in the longrange limit. Values larger than unity indicate that the chance of finding a neighbor at that distance is larger than what would be the case if the atoms (for a fluid with the same density) would be distributed randomly. Figure 4.5 shows the RDF for a WCA fluid at different densities. Due to the finite size of the particles, the function is zero until $r \approx 0.97$. All densities show a peak at a distance around $r \approx 2^{1 / 6}$. This distance corresponds to a minimum potential energy between two LJ atoms and the shortest distance without a repulsive force between LJ and WCA atoms. The RDF corresponding to dense liquids $\rho \geq 0.60$ show multiple peaks that decay with distance. If the density increases past the solid-liquid phase transition line (around $0.92 \leq \rho \leq 0.96$ ), a solid is formed. The RDF of a solid shows very distinct peaks at distances that are related to lattice spacing and lattice type. Since interactions in a lattice have a preferred orientation, a function of only distance is not sufficient anymore to describe the microscopic structure in the solid.

Under the influence of a small field, the orientations of interactions and the preferred distances between particles becomes a function of the direction in the plane of flow 21]. The non-equilibrium pair distribution $g(\mathbf{r}, \nabla \mathbf{u})$ can be related to the radial distribution function [193].

Figure 4.6 shows the distribution of pair interactions in the plane of flow between atoms under various planar flow types. The color is a measure for the normalized probability to find an atom relative to the atom in the middle of the figure. The red color corresponds to a high probability, whereas dark blue is zero probability. The 
fluid is isotropic in equilibrium, hence the distributions show no directional bias in Figure 4.6(a). The black circle in the figure has a radius of $2^{1 / 6}$, which corresponds to the cut-off radius of the WCA potential. Averaging the spherically symmetric distribution over the angle results in the radial distribution function. Figures 4.6(b), 4.6(c) and $4.6(\mathrm{~d})$ show what happens to the pair distribution function under shear, elongational and mixed flow, respectively. The black lines denote the principal orientation of the strain-rate tensor. In addition to the orientations of the pair distribution function and the strain rate tensor, one can look at the principal orientation of the pressure tensor. These directions are generally close to each other, but by no means identical. Note that the pressure tensor calculation uses the same distances between particles are those in the pair distribution function, but now each contact is multiplied with a weight factor, being the interaction force. Furthermore, the principal orientation of the pressure tensor might be slightly affected by the choice of the cut-off distance.

The relation between the hydrostatic pressure and the radial distribution function for a simple fluid in equilibrium is given by 192

$$
p=\frac{N \mathrm{k}_{B} T}{V}-\frac{2 \pi N^{2}}{3 V^{2}} \int_{0}^{\infty} \frac{d U}{d r} r^{3} g(r) d r,
$$

where $U$ is the interaction potential. The first term on the right-hand-side represents the kinetic contribution to the pressure, whereas the second term represents the configurational contribution. Note that this expression is equal to the virial expression (Eq. 2.21), since the integral over the radial distribution function times the gradient of the interaction potential is the same as the product of the interaction distance and the corresponding interaction force averaged over all interactions.

A modified version of Eq. 4.19) can be derived to describe the pressure tensor for a fluid under flow in terms of the non-equilibrium pair distribution function 2 The distortion of the pair distribution function is believed to be related to non-Newtonian behavior in simple fluids [52. Many studies have engaged in relating the distortion of the distribution function to normal stress differences and to relaxation times of a fluid. The basis for these studies is the fact that the normal stress differences and the distortion of the distribution function both follow from a change in the microstructure of the fluid and the pair distribution function contains the required information about the distribution of interaction distances as well as their directions.

Clark and Ackerson were the first to observe shear-induced distortion in the structure factor of a suspension of interacting colloidal particles [197. The authors observed a surprising difference between the principal orientation of the structure factor and the strain rate tensor. Shortly after, Siegfried Hess [198 201] derived a tensorial expansion for the pair correlation function to tensor rank 2, also referred to as the anisotropy

\footnotetext{
${ }^{2}$ This goes beyond the scope of this thesis, but is discussed in Refs. 194 196.
} 


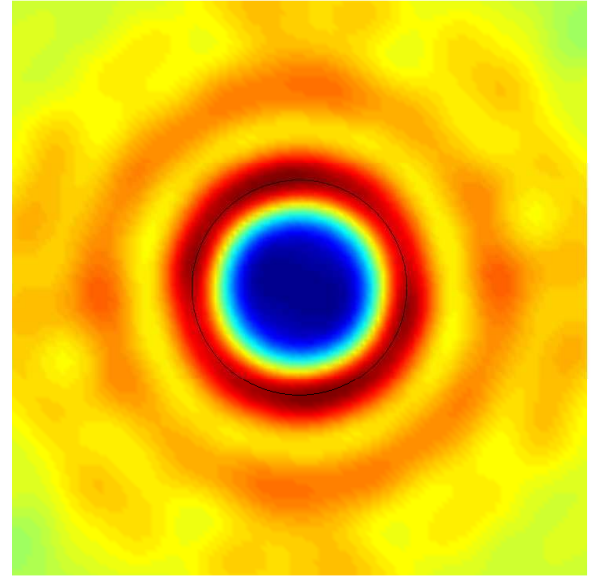

(a)

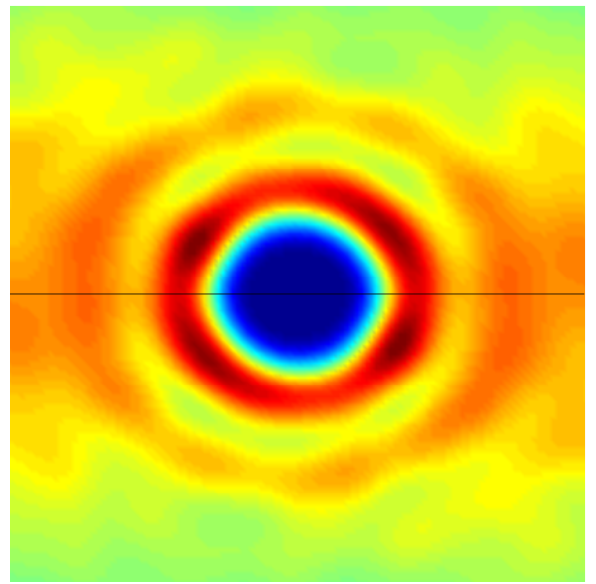

(c)

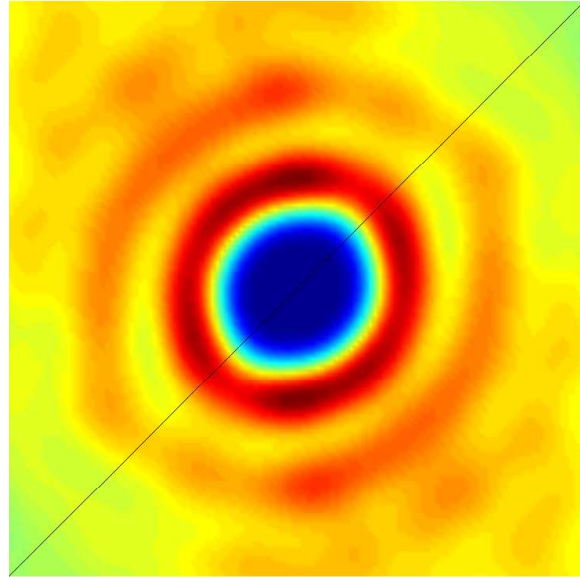

(b)

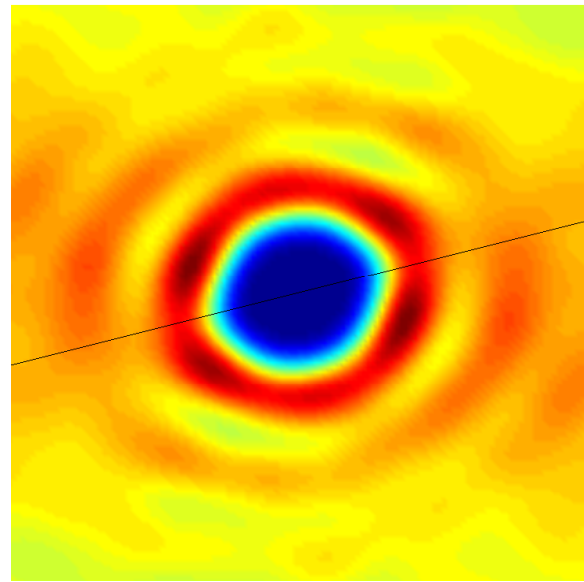

(d)

Figure 4.6: The non-equilibrium pair correlation function for a WCA fluid with $\rho=$ 0.8442 and $T=0.722$ : (a) in equilibrium, (b) shear flow with $\dot{\gamma}=1.0$, (c) planar elongational flow with $\dot{\epsilon}=0.5$ and (d) planar mixed flow with $\dot{\gamma}=1.0$ and $\dot{\epsilon}=0.5$. The black circle in (a) has a radius of $2^{1 / 6}$ and the black lines in the other figures denote the principal orientation of the strain-rate tensor. 
tensor. Furthermore, a differential equation was presented that related the coefficients of the expansion to a phenomenological relaxation time of the fluid. Hanley et al. 193 concluded later, from simulation results, that a higher-rank expansion is needed to describe the microstructure of a fluid under a large shear rate. They extended the expansion to rank 4 and modified the differential equation accordingly. The authors suggested that this model is too simple and that a shear-rate dependent relaxation time might be more suitable.

Many more studies have been performed over the last decades [46, 124, 202, 204]. Besides the microstructure in sheared simple fluids, similar studies have engaged in studying-shear induced structure in plasmas [205] and simple magnetic ferrofluids [206], bond angles in liquid chlorine [207] and collision angles in granular materials [208]. 


\section{5}

\section{Non-Newtonian pressure tensors for simple atomic fluids}

Ever[simple atomic fluids are known to deviate from perfect Newtonian behavior under deformations at a sufficiently high frequency or shear rates. Examples of such deviations are the presence of viscoelasticity, shear thinning, shear dilatancy and normal stress differences [28, 52. It has also been confirmed experimentally for Xenon under shear flow, that viscoelastic behavior occurs for a fluid kept at a constant temperature and pressure [209, 210]. Linear transport coefficients, like the zero-shear rate viscosity, characterize the Newtonian regime, while the shear-rate dependent viscosity and the first and second normal stress coefficients characterize non-Newtonian behavior at higher shear rates. The stress relaxation function is an important property from which we can calculate various material constants. For example, shear viscosity, normal stress coefficients, relaxation time and the infinite-frequency shear modulus. The behavior of the stress relaxation function depends on the state point of the fluid, and so do the quantities that are derived from it. Its behavior as the density is increased from the ideal gas limit to the dense fluid is of great interest, not only because of its importance in studies of transport phenomena, but also in relation to the onset of the glass transition and pre-transitional signatures of solidification [183. It is also desirable to determine whether the stress relaxation function can be modeled with simple functional forms over the entire fluid density range. Although the functional form of the stress relaxation function for several simple liquids has been studied before at var-

This chapter is based on:

R. Hartkamp, P. J. Daivis, and B. D. Todd (2013), Physical Review E 87, 032155,

R. Hartkamp, B. D. Todd, and S. Luding (2013), submitted to J. Chem. Phys. 
ious temperatures and densities, there have been few comprehensive and systematic studies of its density dependence for a single system. A notable exception is the work by Heyes et al. 211, 212, whose main purpose was to study the behavior of the stress relaxation function for atomic fluids with steeply repulsive soft sphere potentials as they approach the hard-sphere limit.

This chapter is organized as follows: Section 5.1 gives a theoretical introduction to material constants and viscoelasticity. Equilibrium molecular dynamics simulation results of a simple atomic fluid over a range of densities are presented in Section 5.2 Next, Section 5.3 presents a non-linear constitutive relation able to predict and quantify some non-Newtonian phenomena. Transient flows are treated in Section 5.4 . Finally, in Section 5.5, our observations are summarized.

\section{$5.1 \quad$ Viscoelasticity}

A constitutive model relates the stress to the flow field. The simplest constitutive model for a compressible viscous fluid is the Newtonian constitutive relation, which assumes a linear relation between the pressure tensor and the rate of deformation

$$
\mathbf{P}=p \mathbf{I}-\eta_{0} \mathbf{S}-\lambda \operatorname{tr}(\mathbf{S}) \mathbf{I}
$$

where $\mathbf{P}$ is the pressure tensor, $p=\frac{1}{3} \operatorname{tr}(\mathbf{P})$ the hydrostatic pressure, $\eta_{0}$ the zero-shear rate viscosity, $\mathbf{S}=\nabla \mathbf{u}+(\nabla \mathbf{u})^{T}$ the symmetric strain rate tensor ${ }^{1} \lambda=\frac{1}{2} \eta_{b}-\frac{1}{3} \eta_{0}$, with $\eta_{b}$ the bulk viscosity and $\mathbf{I}$ is the identity tensor.

In a Newtonian fluid under simple shear flow in the $x-y$ plane, the shear stress is proportional to the strain rate

$$
P_{x y}=-\eta_{0} \dot{\gamma}
$$

This linear relation between stress and strain-rate is a good approximation for many fluids at small deformation rates. However, complex fluids, and even simple fluids at large shear rates, often exhibit a variety of non-Newtonian phenomena, such as shear thinning, shear dilatancy [51, viscoelasticity and normal stress differences [52]. In Chapter 4, we have already seen an example of a shear thinning fluid, where the shear viscosity decreases with increasing strain rate, thus $\eta(\dot{\gamma}>0) \leq \eta_{0}$. This is perhaps the most studied and well-understood deviation from Newtonian behavior. Another possibility is that the steady-state behavior is Newtonian, but the transient stress does not instantaneously satisfy Eq. 5.2. When this is the case, the fluid is said to be viscoelastic.

\footnotetext{
${ }^{1}$ Note that in some literature the strain rate tensor is defined as $\mathbf{S}=\frac{1}{2}\left(\nabla \mathbf{u}+(\nabla \mathbf{u})^{\mathrm{T}}\right)$.
} 


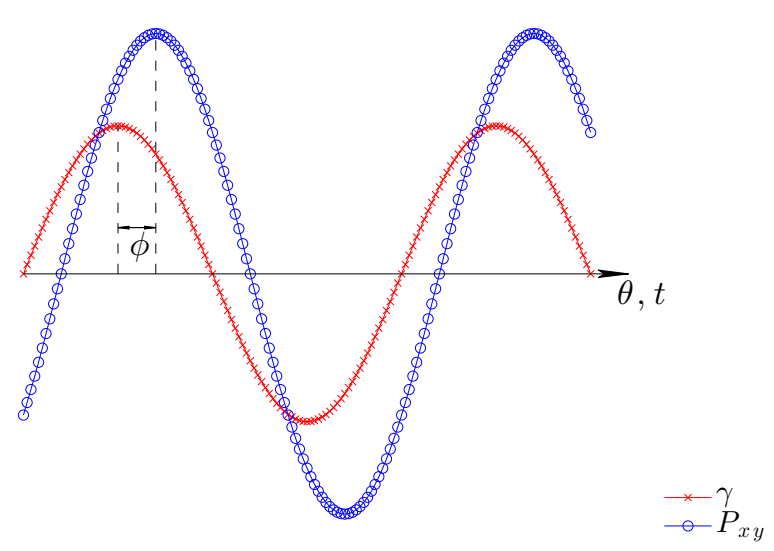

Figure 5.1: The viscoelastic stress response to a sinusoidally varying strain.

\subsubsection{Introduction to viscoelasticity: Cyclic deformation}

An easy way to comprehend viscoelastic theory, is to consider the shear stress response to a sinusoidally varying shear deformation

$$
\gamma(t)=\gamma_{0} \sin (\omega t)
$$

where $\omega$ is the frequency and $\gamma_{0}$ is the magnitude of the cyclic deformation. Differentiating Eq. 5.3 with respect to time leads to the expression for the strain rate

$$
\dot{\gamma}(t)=\omega \gamma_{0} \cos (\omega t)
$$

The shear stress in a viscoelastic fluid follows the cyclic deformation with a delay

$$
P_{x y}(t)=P_{x y, 0} \sin (\omega t+\phi)
$$

where $0 \leq \phi \leq \frac{\pi}{2}$ is the phase lag with respect to the deformation field. The value of $\phi$ depends on the fluid as well as on the frequency of the deformation. Figure 5.1 gives a typical stress response, where the stress lags behind the deformation field. The limiting case corresponds to a perfectly elastic solid, which has zero phase lag $\phi=0$ (such that $P_{x y} \propto \gamma$ ), and a perfectly viscous fluid, with a maximum phase lag $\phi=\frac{\pi}{2}$ (such that $P_{x y} \propto \dot{\gamma}$ ). In the latter case, the shear stress response can be written as

$$
P_{x y}(t)=P_{x y, 0} \sin \left(\omega t+\frac{\pi}{2}\right)=P_{x y, 0} \cos (\omega t) .
$$

This shows that the stress response of an ideal viscous fluid is proportional to the strain rate (Eq. (5.4)). 
Most materials are not perfectly elastic or viscous, they have a finite phase lag smaller than $\phi<\frac{\pi}{2}$. These materials are said to be viscoelastic. If the amplitude of the deformation is small, we can assume a linear stress response, and the elastic and viscous part of the response can be simply added.

Figure 5.2 shows Lissajous figures for the stress-strain and stress-strain-rate relation in time. The stress-strain relation for a perfectly elastic solid is described by a straight line, whereas a viscous fluid follows a ellipse with its height equal to $2 P_{x y, 0}$ and its width $2 \gamma_{0}$. The surface of the ellipse denotes the amount of energy dissipated, which is known as hysteresis. It can be shown that the energy dissipation per cycle (work: $W)$ is given by

$$
\begin{aligned}
W & =\int_{0}^{2 \pi / \omega} P_{x y}(t) \dot{\gamma}(t) d t \\
& =\int_{0}^{2 \pi / \omega} P_{x y, 0} \gamma_{0} \omega \sin (\omega t+\phi) \cos (\omega t) d t \\
& =\pi P_{x y, 0} \gamma_{0} \sin (\phi) .
\end{aligned}
$$

In the relation between stress and strain rate, the viscous fluid forms a straight line, confirming that the shear stress is linearly proportional to strain rate.

The stress-strain and stress-strain-rate relations of the viscoelastic fluid describe an ellipse with a preferred orientation equal to the straight lines ${ }^{2}$ The surface of the ellipse in the stress-strain figure is smaller than for the perfectly viscous fluid. The fact that the ellipse is not symmetric with the strain or strain-rate axis implies that knowledge of the history of the fluid is required to predict the current stress in the material. This point will become more clear later on.

The ability of a material to store energy is given by the storage modulus $G^{\prime}$, which is based on the in-phase stress and thus related to the elastic response of the fluid. The ability to dissipate energy is related to the out-of-phase stress, that is the viscous response and is given by the loss modulus $G^{\prime \prime}$

$$
\begin{aligned}
G^{\prime} & =\frac{P_{x y, 0}}{\gamma_{0}} \cos (\phi), \\
G^{\prime \prime} & =\frac{P_{x y, 0}}{\gamma_{0}} \sin (\phi) .
\end{aligned}
$$

The amount of lag is related to the ratio between the storage and loss moduli

$$
\tan (\phi)=\frac{G^{\prime \prime}}{G^{\prime}}
$$

\footnotetext{
${ }^{2}$ This is only true for small values of $\gamma_{0}$, otherwise the shape loses its symmetry with respect to the orientation of the elastic stress-strain relation, as shown for example in Refs. 213] 214].
} 


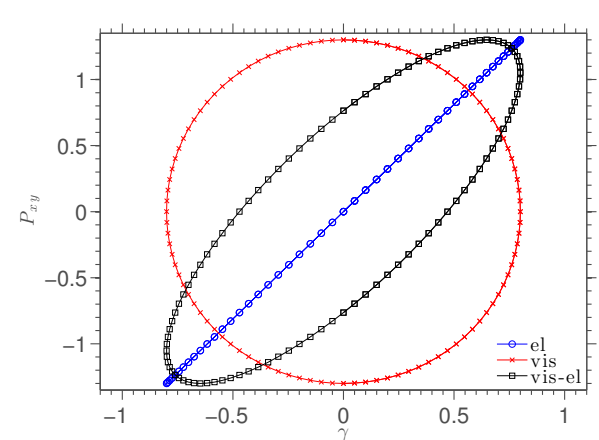

(a)

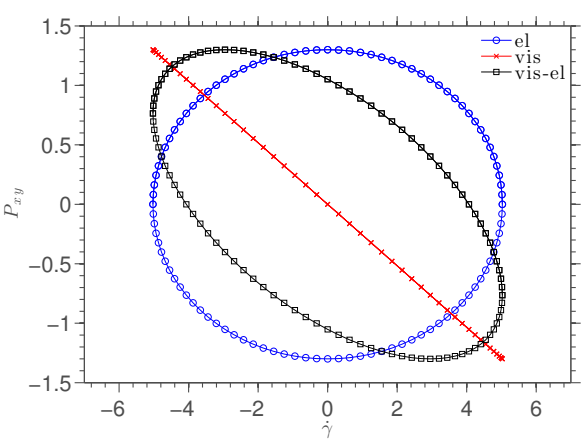

(b)

Figure 5.2: Stress-strain (a) and stress-strain-rate (b) relations for an elastic, viscous and viscoelastic material.

In the limiting case of perfectly elastic solid, zero phase lag results in $G^{\prime}=P_{x y, 0} / \gamma_{0}$ and $G^{\prime \prime}=0$. The other limit corresponds to a perfectly viscous fluid, with a phase lag $\phi=\pi / 2$. In that case the moduli are $G^{\prime}=0$ and $G^{\prime \prime}=P_{x y, 0} / \gamma_{0}$.

Eq. (5.5) can be rewritten using the rules of trigonometry

$$
\begin{aligned}
P_{x y}(t) & =P_{x y, 0}(\cos (\phi) \sin (\omega t)+\sin (\phi) \cos (\omega t)) \\
& =\gamma_{0}\left(G^{\prime} \sin (\omega t)+G^{\prime \prime} \cos (\omega t)\right) .
\end{aligned}
$$

The strain on a fluid is often not just an oscillating function. Consequently, an expression of the shear stress as a function of time cannot be easily derived in practice.

\subsubsection{Material functions for viscoelastic fluids}

In linear viscoelastic fluids, the responses are additive. The stress response for a fluid subjected to a small strain can then be described by

$$
P_{x y}(t)=-G \gamma(t)-\eta \dot{\gamma}(t)
$$

where $\eta$ is the shear viscosity and $G$ is the (shear) stress relaxation modulus, which is defined by the stress-strain ratio at a constant deformation rate.

The instantaneous shear stress in a viscoelastic fluid depends on the present deformation rate as well as on the full history of the flow. The shear stress in a sheared fluid is given by the following expression ${ }^{3}$

$$
P_{x y}(t)=-\int_{-\infty}^{t} G\left(t-t^{\prime}\right) \dot{\gamma}\left(t^{\prime}\right) d t^{\prime} .
$$

\footnotetext{
${ }^{3}$ Theoretical treatment of convolution products like this is often easier in the frequency-domain, where Eq. 5.16 simply reads $P_{x y}(\omega)=G^{*}(\omega) \dot{\gamma}(\omega)$.
} 
This is the most general linear constitutive relation for a viscoelastic fluid [52]. If a strain rate is activated at $t=0$, we can rewrite Eq. (5.16), using $\dot{\gamma}(t)=\dot{\gamma} H(t)$, where $H$ is the Heaviside function

$$
\begin{aligned}
P_{x y}(t)= & -\int_{0}^{t} G\left(t-t^{\prime}\right) \dot{\gamma}\left(t^{\prime}\right) d t^{\prime} \\
& =-\dot{\gamma} \int_{0}^{t} G\left(t-t^{\prime}\right) d t^{\prime} .
\end{aligned}
$$

Similarly, to calculate the shear stress after the cessation of steady shear, we can apply a change of the variable of integration $t-t^{\prime}=s$ and derive

$$
P_{x y}(t)=-\dot{\gamma} \int_{t}^{\infty} G(s) d s .
$$

The rheological behavior of a non-equilibrium fluid can be described by shear-rate dependent viscometric functions. For example, shear viscosity is given by

$$
\eta(\dot{\gamma})=-\frac{P_{x y}}{\dot{\gamma}}
$$

and the first and second normal stress coefficients are given by 99

$$
\begin{aligned}
& \Psi_{1}(\dot{\gamma})=\frac{\mathcal{N}_{1}}{\dot{\gamma}^{2}}=\frac{P_{y y}-P_{x x}}{\dot{\gamma}^{2}}, \\
& \Psi_{2}(\dot{\gamma})=\frac{\mathcal{N}_{2}}{\dot{\gamma}^{2}}=\frac{P_{z z}-P_{y y}}{\dot{\gamma}^{2}},
\end{aligned}
$$

where $\mathcal{N}_{1}$ and $\mathcal{N}_{2}$ denote the first and second normal stress differences, respectively. In the limit of zero-shear rate, the viscosity converges to the zero-shear rate viscosity

$$
\eta_{0}=\lim _{\dot{\gamma} \rightarrow 0} \eta(\dot{\gamma})
$$

and the normal stress coefficients approach (typically non-zero) values, called the zeroshear rate normal stress coefficients

$$
\Psi_{\alpha, 0}=\lim _{\dot{\gamma} \rightarrow 0} \Psi_{\alpha}(\dot{\gamma}), \quad \alpha=1,2
$$

The second normal stress coefficient $\Psi_{2}$ is far less often studied than $\Psi_{1}$, because it is often much smaller and thus more difficult to measure accurately in the case of polymeric liquids [215]. It is also more difficult to obtain computationally, because there is currently no known method of obtaining it from an equilibrium molecular dynamics (EMD) simulation. In this thesis, we will focus on the first normal stress coefficient, rather than the second. 
Rather than extracting the normal stress coefficient from non-equilibrium simulations and taking the zero-shear rate limit, it can be calculated by evaluating an expression in equilibrium, derived by Coleman and Markovitz 216]

$$
\Psi_{1,0}=2 \int_{0}^{\infty} t G(t) d t .
$$

For an isotropic system, the stress relaxation modulus can be calculated as [185]

$$
G(t)=\frac{V}{10 \mathrm{k}_{\mathrm{B}} T}\left\langle\mathbf{P}^{0 s}(t): \mathbf{P}^{0 s}(0)\right\rangle,
$$

where $V$ is the system volume, $\mathrm{k}_{\mathrm{B}}$ Boltzmann's constant, $T$ the temperature, the superscript " $0 s$ " denotes a traceless and symmetric tensor, which contains 5 independently fluctuating quantities. While this formulation is identical to the shear stress autocorrelation function that is commonly used as the susceptibility of the Green-Kubo shear viscosity integral, the tensorial approach is preferred for its enhanced statistics [217]. The pressure tensor is given by

$$
\mathbf{P}=\frac{1}{V} \sum_{i=1}^{N}\left(\frac{\mathbf{p}_{i} \mathbf{p}_{i}}{m_{i}}+\frac{1}{2} \sum_{j \neq i} \mathbf{r}_{i j} \mathbf{F}_{i j}\right) .
$$

where $\mathbf{p}_{i}$ is the peculiar momentum of particle $i, \mathbf{r}_{i j}=\mathbf{r}_{i}-\mathbf{r}_{j}, r=\left|\mathbf{r}_{i j}\right|$ and $\mathbf{F}_{i j}$ is the force on particle $i$ due to particle $j$. The first term on the right-hand side is the kinetic part of the pressure tensor, whereas the second term is the configurational part.

The SACF of the traceless symmetric pressure tensor for a dense fluid is known to depend mostly on the configurational part [218] and thus depends strongly on the interaction potential. Heyes [76] calculated the dependence of several properties on the exponent in strongly repulsive potentials. He found that the infinite-frequency shear modulus increased approximately linearly with the exponent of the potential. Powles and Heyes [211] showed that in the hard-sphere limit (i.e., for steeply repulsive potentials), the shear stress and pressure correlation functions become simple analytic functions of the temperature and density. Furthermore, Brańka and Heyes [212] showed how the stress autocorrelation function depends on the exponent in strongly repulsive potentials. They showed that the decay time of the correlation function scales with the exponent of the interaction potential.

Viscoelastic fluids show a combination of elastic (solid-like) and viscous (liquid-like) stress response when a deformation is applied. The elastic response is proportional to the strain $\gamma$, with the elastic modulus $G$ as the proportionality constant. The shear viscosity $\eta$ is the proportionality constant relating the shear stress to the strain rate $\dot{\gamma}$. When a viscoelastic fluid is perturbed, it initially responds only elastically. The lag of the viscous response is related to the relaxation mechanism in viscoelastic fluids. In 
a steady state (zero-frequency perturbation) flow, the elastic energy is stored during the approach to steady state. The real part of the shear modulus is zero for a fluid in the zero-frequency limit [219, 220]. On the other hand, when a fluid is perturbed at very high frequencies, the elastic constant has a non-zero value. The infinite-frequency shear modulus $G^{*}(\omega \rightarrow \infty) \equiv G^{\infty}$ is a common measure for the elastic part of the response. This value corresponds to the initial value of the stress autocorrelation function $G^{\infty}=G(t=0)=V\left\langle\mathbf{P}^{0 s}(0): \mathbf{P}^{0 s}(0)\right\rangle /\left(10 \mathrm{k}_{\mathrm{B}} T\right)$ [192, 221, 222].

Similarly to the first normal stress coefficient (Eq. (5.25)), the zero-shear rate viscosity can be calculated from the stress relaxation modulus

$$
\eta_{0}=\int_{0}^{\infty} G(t) d t
$$

which is the Green-Kubo relation for shear viscosity. Using Eqs. (5.25) and (5.27), the viscous relaxation time is given by

$$
\tau=\frac{\Psi_{1,0}}{2 \eta_{0}}=\frac{\int_{0}^{\infty} t G(t) d t}{\int_{0}^{\infty} G(t) d t}
$$

This expression makes no assumptions about the properties of the fluid or the shape of the relaxation function, apart from convergence of the relevant integrals. This expression differs from the definition of the Maxwell relaxation time, $\tau_{M}=\eta / G_{\infty}$ 223. Maxwell assumed an exponential decay of the stress autocorrelation function, which is appropriate at long times for dilute gases, but is not an accurate approximation for dense fluids. The Maxwell relaxation time is given by

$$
\tau_{M}=\frac{\int_{0}^{\infty} G(t) d t}{G(0)} .
$$

In fact, a hierarchy of relaxation times can be defined, each one differing in the order of $t$ of the numerator and denominator, with the ratio remaining of order $t$. The Maxwell time can therefore be seen as a zeroth-order relaxation time, and the one defined in Eq. (5.28) can be seen as a first order relaxation time. The highest order relaxation time that can be evaluated depends on the convergence properties of the integrals. For an exponential stress relaxation function, the relaxation times of all orders exist, and they are all equal to the Maxwell time. However, not all stress relaxation functions are exponential in time. 


\subsection{Calculation of material constants from EMD sim- ulations}

Most studies of the viscoelastic properties of simple fluids have concentrated on the viscosity, the infinite-frequency shear modulus and the Maxwell relaxation time. The first normal stress coefficient has received much less attention because it is more difficult to compute. The viscosity is usually calculated using EMD simulations and the Green-Kubo [186, 187] relation that gives the viscosity in terms of the integral of the stress autocorrelation function (SACF). The shear relaxation function (often known as the shear relaxation modulus in the rheology literature) is directly proportional to the SACF.

The viscoelastic relaxation of simple fluids has been studied by a number of authors [24, 182, 183, 224, 227. Mountain and Zwanzig [225] studied the density-dependence of the Maxwell relaxation time of a supercritical Lennard-Jones fluid. They found a decrease in relaxation time with increasing density up to the maximum density studied, $\rho=0.7$. More recently, Keshavarzi et al. [227] studied the density- and temperature dependence of the infinite-frequency shear modulus and the Maxwell relaxation time for purely repulsive soft sphere and Lennard-Jones fluids. They calculated the infinitefrequency shear modulus numerically from the equilibrium radial distribution function, using the relation derived by Zwanzig and Mountain [192]. A minimum relaxation time was again found around a reduced density of $\rho \approx 0.7$, independent of the temperature. The authors argued that this is a transition point, below which kinetic momentum transport dominates and above which the finite memory of the fluid dominates the flow behavior.

Little computational work has been done on calculating the zero-shear rate normal stress coefficients 185, but measurements of these quantities are common in rheological experiments [213, 228, 229]. Loose and Hess [230] computed the non-equilibrium shear viscosity and normal stress coefficients for a moderately dense Lennard-Jones gas ( $\rho=0.1, T=2.75$ ) over a range of shear rates by homogeneous non-equilibrium molecular dynamics (NEMD). Their data show large statistical uncertainty for low strain rates, but their results for the shear rate dependent viscosity and the normal stress coefficients agree well with kinetic theory as shown in Ref. [231]. Although their system was a gas of moderate density, its behavior was well approximated by the ideal gas model because the state point chosen was close to the Boyle point, where the effects of attractive and repulsive interactions approximately cancel each other.

Coleman and Markovitz [216] derived an expression for the zero-shear rate first normal stress coefficient in terms of the linear stress relaxation modulus from a continuum theory of non-linear viscoelasticity. Their expression is well-known and verified 
experimentally in polymer rheology [215]. Calculation of the first normal stress coefficient using their method with EMD simulations is computationally expensive, even with modern computers, because it requires stress autocorrelation function data of extremely high precision. Recently, Daivis [185] used the Coleman-Markovitz method to calculate the zero-shear rate normal stress coefficient of a simple liquid. He also calculated the zero-shear rate viscosity and first normal stress coefficient using NEMD by extrapolating the shear-rate dependent viscosity and first normal stress coefficient to zero-shear rate, for a range of densities. While the equilibrium and non-equilibrium calculations of the viscosity agreed in the limit of zero-shear rate, the values of the first normal stress coefficient did not. A detailed understanding of this discrepancy is still lacking, but it is clear that the choice of thermostat has a strong influence on the values of non-linear rheological properties calculated in NEMD simulations [102].

The shear relaxation function completely determines the linear viscoelastic shear response of a fluid. Enskog kinetic theory predicts that at low density, the stress autocorrelation function for a hard-sphere fluid should be a delta function at zero time due to the impulsive nature of hard-sphere forces, followed by an approximately single exponential decay 24]. The delta function results in an infinite value of the infinitefrequency shear modulus for the hard-sphere fluid at all densities, whereas the viscosity remains finite. In this case, the Maxwell relaxation time is zero, which would seem to imply that the stress relaxes to its steady state value instantaneously after the onset of steady shear and that viscous flow of a hard-sphere fluid is perfectly inelastic. However, this is clearly not the case, because the kinetic part of the hard-sphere stress relaxation function has a non-zero relaxation time, and the overall relaxation of the SACF does not take place instantaneously. In this sense, the Maxwell time is a misleading measure of viscoelastic relaxation time. An alternative definition of the viscoelastic relaxation time, based on the ratio of the first normal stress coefficient to the viscosity, does not exhibit this anomaly.

\subsubsection{Simulation details}

We simulate an atomic fluid whose interactions are mediated via a Weeks-ChandlerAndersen (WCA) 77] potential and the equations of motion are integrated with the Gear predictor-corrector algorithm with a time-step of $\Delta t=0.001$ in reduced units. All physical quantities presented are reduced using the particle mass $m$, interaction length scale $\sigma$ and the potential energy well-depth $\epsilon$, as shown in Chapter 2 .

The equilibrium simulations in this work each start from a lattice containing 4000 atoms. The fluid is then equilibrated until a steady-state is reached. After the equilibration, correlation function calculations are started, with a maximum lag-time $t=10$ in reduced units. We average the correlation function over $6.0 \times 10^{7}$ trajectories to 
gather enough accumulations. This number is extremely large because an accurate calculation of the zero-shear first normal stress coefficient requires a lot of statistics. Each simulation in this work is performed at a temperature $T=1.0$, where a Gaussian isokinetic thermostat 114 is used to control the temperature of the fluid.

\subsubsection{Simulation results}

The infinite-frequency shear modulus, zero-shear rate normal stress coefficient and the relaxation time are calculated from the equilibrium stress autocorrelation function, by applying the theory presented in Section 5.1 to our MD simulations.

Figure 5.3 shows the zero-shear rate viscosity, the zero-shear rate first normal stress coefficient and the viscous relaxation time for a fluid in equilibrium at a density $\rho=$ 0.84 and temperature $T=1.0$. Comparing both profiles illustrates the large amount of averaging needed for accurate calculations of the zero-shear rate normal stress coefficient. The zero-shear rate normal stress coefficient converges to a value within error bars of the value calculated by Daivis [185] (he calculated $\Psi_{1,0}=0.32 \pm 0.10$ ) and higher than the values obtained from extrapolating NEMD results. The latter could be related to an unknown shear-dependence of the normal stress coefficient at extremely low shear rate. However, it seems more likely that the action of the homogeneous thermostat is responsible for a discontinuous change in the first normal stress coefficient from the equilibrium value. As discussed in Ref. [185, the temperature is not well-defined out of equilibrium and the calculation of second-order properties are sensitive to the thermostatting mechanism used, because the normal stress differences and the non-equilibrium corrections to the temperature are both of $\mathcal{O}\left(\dot{\gamma}^{2}\right)$. The relaxation time can be calculated using Eq. (5.28). Since the viscosity integral quickly converges to a constant value, the profile for the relaxation time (Figure 5.3(c) looks much the same as the zero-shear rate first normal stress coefficient, shown in Figure 5.3(b). The viscous relaxation time obtained at the current state point is $0.098 \pm 0.002$.

The stress relaxation modulus for a WCA fluid at densities $\rho=0.08,0.12,0.20$, $0.28,0.36,0.44,0.52,0.60,0.68,0.76,0.84$. 0.88 and 0.92 are shown in Figure 5.4 . Each of the stress relaxation functions show the same behavior at small times. This part of the correlation function is related to the fact that at short times the relative positions and velocities of atoms have not been changed dramatically by interactions and diffusion, most atoms are still within the available free space that was directly surrounding them at $t=0$. The relaxation modulus has a zero slope at $t=0$, which is directly followed by a Gaussian-like fast decay. This can be understood by considering a power series expansion of the autocorrelation function $C_{P P}(t)=\left\langle\mathbf{P}^{0 s}(t): \mathbf{P}^{0 s}(0)\right\rangle$ 


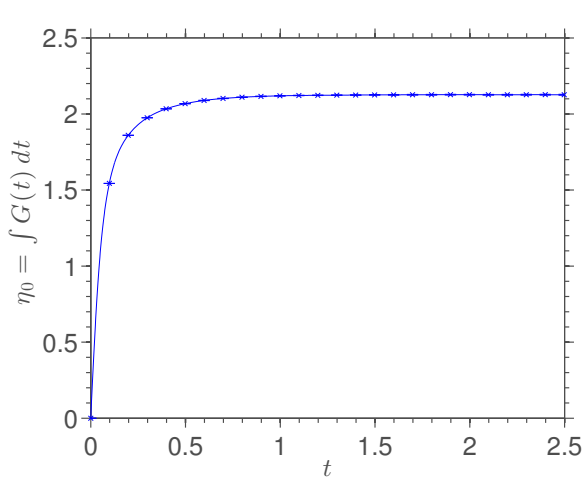

(a)

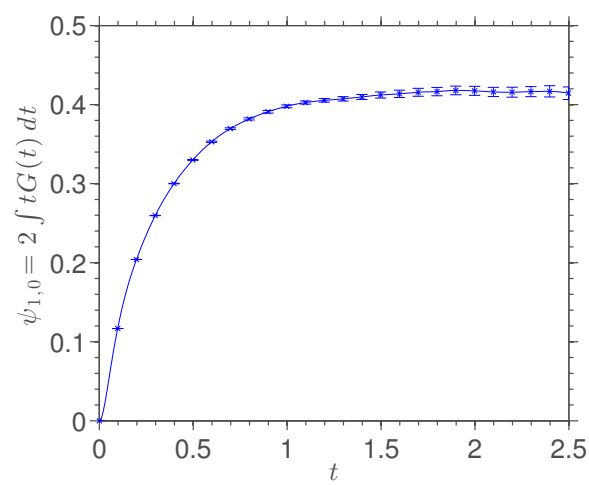

(b)

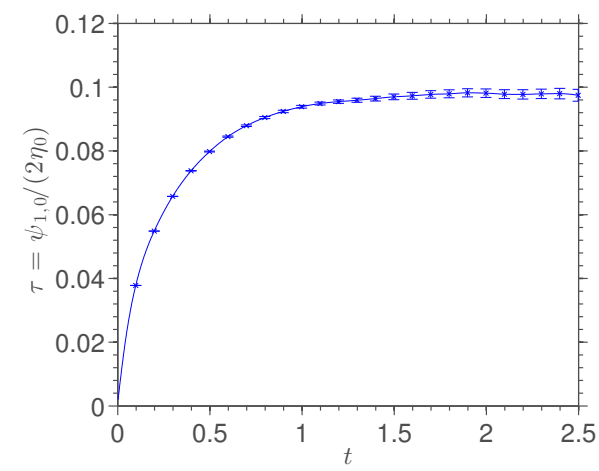

(c)

Figure 5.3: Shear viscosity, normal stress coefficient and relaxation time. The data is averaged over $6.0 \times 10^{7}$ trajectories. The fluid has a density $\rho=0.84$ and temperature $T=1.0$. 


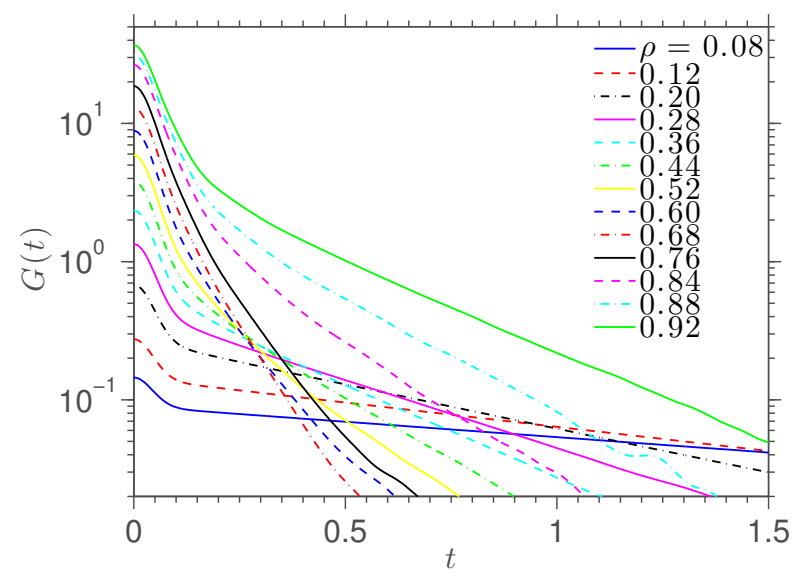

Figure 5.4: The stress relaxation modulus at different densities and a temperature $T=1.0$. Each stress relaxation functions is averaged over $6.0 \times 10^{7}$ trajectories. $G(0)$ increases monotonically with increasing density.

around $t=0$ 63.

$$
C_{P P}(t)=C_{P P}(0)-\frac{t^{2}}{2 !}\left\langle\dot{\mathbf{P}}^{0 s}(0): \dot{\mathbf{P}}^{0 s}(0)\right\rangle+\frac{t^{4}}{4 !}\left\langle\ddot{\mathbf{P}}^{0 s}(0): \ddot{\mathbf{P}}^{0 s}(0)\right\rangle+\mathcal{O}\left(t^{6}\right) .
$$

This expansion has a similar functional form as a power series expansion of a Gaussian function.

At longer times, the decay of the kernel depends strongly on the density. The rate of decay of the kernel decreases with the density at low densities $\rho \leq 0.68$, whereas the opposite trend is observed at higher densities $\rho>0.68$. The memory of a fluid decays in time due to interactions between atoms. If the fluid is dilute, few interactions occur, which results in a very slow decay of the memory, i.e., very long relaxation times, this becomes less true as the fluid is more dense, such that the decay-rate of the correlation function increases. However, if the fluid becomes too dense $(\rho>0.68)$, the environment of the atoms in an equilibrium fluid does not change much due to a very low self-diffusion coefficient [232]. This causes the decay-rate of the correlation function to decrease again towards the dense-fluid limit.

In the dilute limit, the shape of the shear modulus can be accurately approximated with the sum of a Gaussian plus an exponential decay

$$
G(t) \approx G^{\infty}\left(A \mathrm{e}^{-t^{2} /\left(2 \tau_{1}^{2}\right)}+(1-A) \mathrm{e}^{-t / \tau_{2}}\right),
$$

where $A$ is a measure of the initial relative magnitude of the Gaussian mode of relaxation, $\tau_{1}^{2}$ is the variance of the Gaussian and $\tau_{2}$ is the relaxation time of the exponentially decaying mode. Powles and Heyes 211] suggested a fitting function, for the 


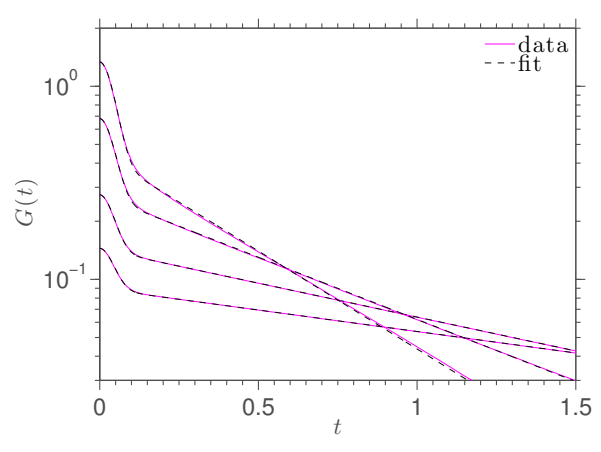

(a)

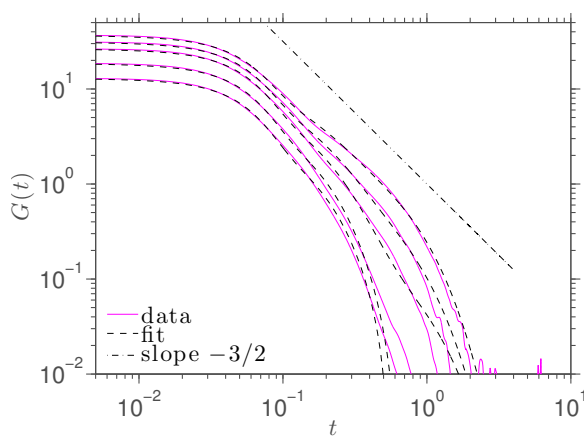

(b)

Figure 5.5: The shear modulus of a dilute (a) and dense (b) fluid. The dilute fluid is fitted with the sum of a Gaussian and an exponential, the curves correspond from bottom to top (left of the crossings) to: $\rho=0.08,0.12,0.20$ and 0.28 . The relaxation functions of dense fluids are fitted with the sum of a Gaussian and two exponentially decaying modes. The stress relaxation functions correspond from bottom to top to: $\rho=0.68,0.76,0.84,0.88$ and 0.92 .

shear stress and pressure autocorrelation functions, of the form $C(t) \propto \operatorname{sech}(\sqrt{2 T} n t)$, where $n$ is the power of the repulsive potential. Since this is an even function in time, it satisfies the form of the Taylor series expansion around $t=0$, given in Eq. (5.30). Furthermore, at long times, this function approaches an exponential decay, such as predicted by the Maxwell model. Brańka and Heyes [212] showed later, for the pressure autocorrelation function, that it is not possible to represent the steeply repulsive behavior of the relaxation function by the suggested functional form, and an additional singular function is required. This makes their fitting function more complicated than the functional form suggested in Eq. 5.31.

Least-squares fits of the shear moduli of a fluid of densities $\rho=0.08,0.12,0.20$ and 0.28 are shown in Figure 5.5(a) and the corresponding fitting parameters are given in Table 5.1. The values for $A$ show an increasing trend with density. This trend implies that only in the dilute limit, will a single exponential (which is, for example, assumed in a Maxwell model) become a reasonable approximation of the stress relaxation modulus. The variance of the Gaussian $\tau_{1}^{2}$ shows no strong density dependence, even for higher densities. We observe that the fitted relaxation times of the exponential mode $\tau_{2}$ decrease with increasing density (for dilute fluids). Since the contribution of the Gaussian decays quickly to zero, the exponential mode gives a good approximation of the relaxation time of the stress relaxation modulus at low densities. As the density increases to $\rho>0.28$, the functional form of the shear modulus is no longer 
well-described by Eq. (5.31). At higher densities $(\rho \geq 0.68)$, the shape of the kernel

Table 5.1: Fitting parameters for the dilute and dense stress relaxation moduli.

\begin{tabular}{cccccc}
$\rho$ & $A$ & $B$ & $\tau_{1}^{2}$ & $\tau_{2}$ & $\tau_{3}$ \\
\hline 0.08 & 0.3814 & - & 0.0016 & 1.9538 & - \\
0.12 & 0.4778 & - & 0.0016 & 1.2362 & - \\
0.20 & 0.5986 & - & 0.0016 & 0.6746 & - \\
0.28 & 0.6635 & - & 0.0017 & 0.4281 & - \\
\hline 0.68 & 0.6218 & 0.8781 & 0.0018 & 0.1270 & 0.1465 \\
0.76 & 0.6050 & 0.7597 & 0.0018 & 0.1197 & 0.1396 \\
0.84 & 0.5890 & 0.3978 & 0.0019 & 0.1051 & 0.4666 \\
0.88 & 0.5785 & 0.3738 & 0.0019 & 0.1016 & 0.3723 \\
0.92 & 0.5664 & 0.3471 & 0.0019 & 0.0976 & 0.3882
\end{tabular}

becomes more difficult to describe. The increasing trend in $A$ at low densities, does not continue at higher densities. Instead, the relative magnitude of the first mode decreases at high density, while the variance of the Gaussian mode shows very little dependence on the density. A long-time tail forms at high densities, which can be assumed to be an additional mode of relaxation. Fits of the high-density relaxation modulus are shown in Figure 5.5(b) The data, from top to bottom, correspond to densities $\rho=0.92,0.88,0.84,0.76$ and 0.68 , respectively. The functional form of the fit is given by the the sum of a Gaussian and two exponential modes

$$
G(t) \approx G^{\infty}\left(A \mathrm{e}^{-t^{2} /\left(2 \tau_{1}^{2}\right)}+B \mathrm{e}^{-t / \tau_{2}}+(1-A-B) \mathrm{e}^{-t / \tau_{3}}\right) .
$$

This functional form results in reasonable fits of the data corresponding to the highest densities $(\rho=0.88$ and 0.92$)$, but is less successful at lower densities. Furthermore, neither of the stress relaxation functions shows a tail that is proportional to $t^{-3 / 2}$ over a large time range. However, the relaxation function corresponding to $\rho=0.84$, shows a decay-rate that is almost equal to $3 / 2$ over a short range of time.

The integral over the shear modulus results in the shear viscosity profile (Eq. (5.27)). Figure 5.6 shows the shear viscosities corresponding to the shear moduli shown in Figure 5.4 The data shows a similar trend to the simulation results from Silva et al. 233] for a WCA fluid and the data from Baidakov et al. 234] and Todd [235] for a Lennard-Jones fluid. The latter shows quantitative differences in the dilute and dense limits. This disagreement is due to the different interaction potential, as illustrated by the results of Cappelezzo et al. [236]. They used a Stokes-Einstein relation to calculate the shear viscosity of simple fluids at equilibrium. Their data, for a WCA 
fluid at density $\rho=0.85$ and temperature $T=1.0$, agrees well with the viscosity of our WCA fluid around that density and the Lennard-Jones fluid shows good agreement with the viscosity of the Lennard-Jones fluid reported by Baidakov et al. and by Todd 4 Furthermore, the authors reported viscosities of a Lennard-Jones fluid and a WCA fluid at a temperature $T=1.5$ over a range of densities. The discrepancy between viscosities of WCA and LJ fluids at this temperature was consistent with the behavior in Figure 5.6 at low densities the WCA fluid has a higher viscosity than the Lennard-Jones fluid, but at high densities it is the other way around. Silva et al. 233. proposed an expression to relate the viscosity of a dense WCA fluid $(\rho \geq 0.6)$ to that of a Lennard-Jones fluid at the same state point. This relation is given by

$$
\frac{\eta_{L J}}{\eta_{W C A}}=\theta_{0}+\frac{\theta_{1}}{T}
$$

where $\theta_{0}$ and $\theta_{1}$ are fitting parameters. The authors found $\theta_{0}=0.9932$ and $\theta_{1}=$ 0.2778 , which means that for a fluid at temperature $T=1.0$ we find $\eta_{L J}=1.271 \eta_{W C A}$ for all densities $\rho \geq 0.6$. This expression gives a reasonable estimate, but in practice the ratio between both viscosities depends on the density as well as the temperature, as shown by the data listed by Silva et al. [233]. This scaling results in good agreement (not shown here) between the Lennard-Jones and the WCA data in Figure5.6.

Rowley and Painter 237] presented a function to describe the dependence of shear viscosity on density and temperature. Since we only consider a single temperature here, we can simplify the model to

$$
\eta(\rho)=\eta(0) \exp \left(\sum_{i=1}^{d} C_{i} \rho^{i}\right),
$$

where $d$ is the degree of the polynomial. The fit, shown in Figure 5.6. shows good agreement for $d=4$. The parameters of the fit are $\eta(0)=0.1797, C_{1}=-0.6175$, $C_{2}=8.2438, C_{3}=-8.8141$ and $C_{4}=4.8280$. The fit could be simplified further to a stretched exponential fit [235]. The stretched exponential function is given by

$$
\eta(\rho)=\eta(0) \exp \left(C_{1} \rho^{C_{2}}\right) .
$$

The model parameters are fitted as $\eta(0)=0.2019, C_{1}=3.4478$ and $C_{2}=2.1433$. This functional form leads to a less accurate fit than Eq. (5.34) with $d=4$, especially in the dilute region.

The zero-density limit viscosity $\eta(0)=0.1797$ that we found from the most accurate fit (Eq. (5.34) ) can be compared to the predicted value from kinetic theory for the zerodensity limit. The theory predicts a zero-shear rate viscosity given by [231]

$$
\eta_{0}=\frac{p}{\nu}
$$

\footnotetext{
${ }^{4}$ We have used the data from Cappelezzo et al. [236] that corresponds to 4096 atoms, since this is closest to our system size.
} 


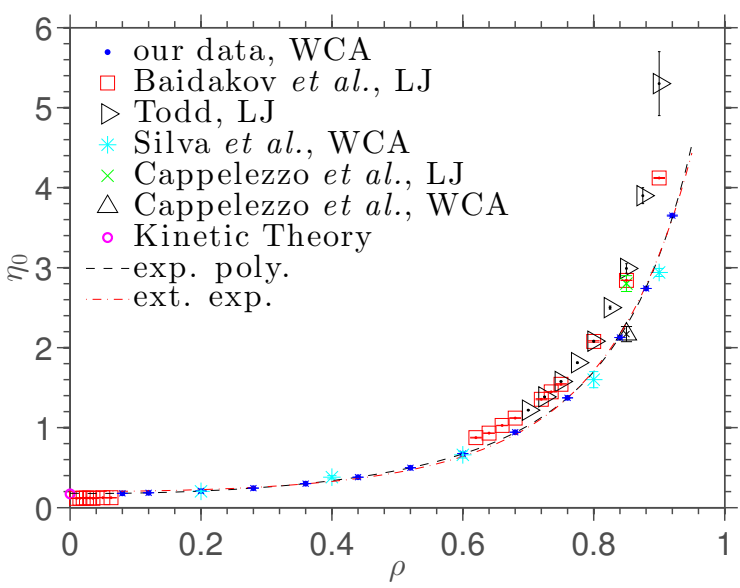

Figure 5.6: The zero-shear viscosity as a function of density, obtained from EMD simulations at a temperature $T=1.0$. The data is compared to the values reported by Silva et al. 233] for a WCA fluid, the data from Baidakov et al. 234] and Todd [235] for a Lennard-Jones fluid and data from Cappelezzo et al. 236] for Lennard-Jones and WCA fluids. Furthermore, our data is fitted with two functions and the zero-density shear viscosity is predicted with kinetic theory.

where $p$ is the hydrostatic pressure and $\nu$ the collision rate. The pressure of a dilute fluid is given (in physical units) by

$$
p=\frac{N \mathrm{k}_{\mathrm{B}} T}{V} .
$$

The collision rate $\nu$ can be expressed in terms of the Lennard-Jones parameters

$$
\nu=\frac{3 N A_{2}}{V}, \quad A_{2}=1.3703 \sqrt{\frac{2 \epsilon}{m}}=1.938,
$$

where the numerical value for $A_{2}$ corresponds to Maxwell molecules [238, which are softer than WCA atoms. However, as discussed in Ref. [231], the collision rate for dilute fluids shows little dependence on the interaction potential used. Substituting the parameters for our simulations, we find a viscosity $\eta_{0}=\mathrm{k}_{\mathrm{B}} T\left(3 A_{2}\right)^{-1}=0.1720$. This result is within $5 \%$ of the value that we found from the fit of the MD simulation results. Furthermore, Rowley and Painter [237] have presented an expression, based on the Chapman-Enskog theory, for the zero-density shear viscosity of a Lennard-Jones fluid. Using their expression for a fluid at temperature $T=1.0$, the zero-density shear viscosity is $\eta(0)=0.1256$. This value is much lower than the WCA data suggests, but more consistent with the Lennard-Jones data from Baidakov et al. [234].

The infinite-frequency shear modulus, as a function of density, is shown in Figure 5.7. The interaction potential used to model the fluid has a strong influence on 


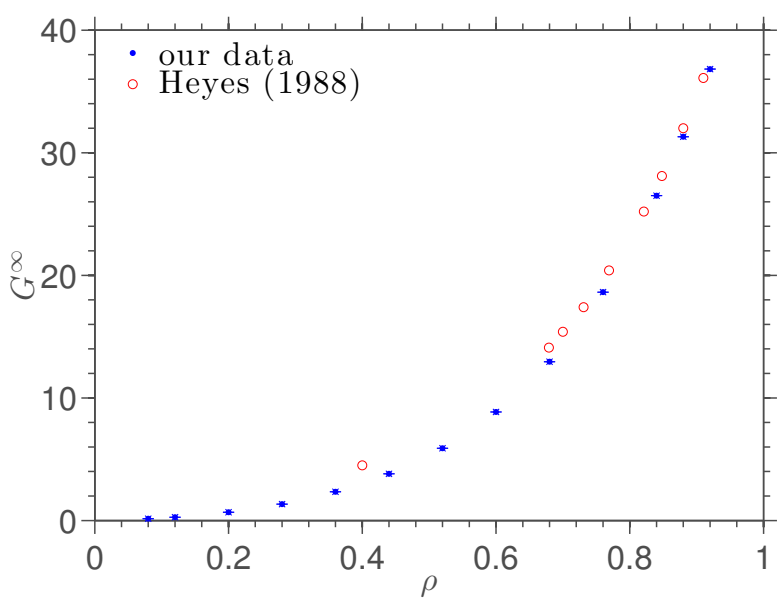

Figure 5.7: The infinite-frequency shear modulus as a function of density, obtained from EMD simulations at a temperature $T=1.0$. The data is compared to the values reported by Heyes 221] for a Lennard-Jones fluid at a temperature $T=1.06$.

the value of the infinite-frequency shear modulus, as mentioned in Section 5.1. In the hard-sphere limit, the modulus goes to infinity for soft-sphere potentials, as can be understood from the integral relation given by Zwanzig and Mountain [192. The nonlinear relation between pressure and density leads to a strong increase in $G^{\infty}$ with increasing density. The modulus approaches zero in the low-density limit, which means that the elastic response becomes negligibly small and the fluid has no notable memory. The infinite-frequency shear moduli for densities $\rho=0.68,0.78$ and 0.88 are compared to the values reported by Heyes 221] for a Lennard-Jones fluid at a temperature $T=1.06$. Good agreement is found for these values, although an exact quantitative comparison is not possible since the author used a different interaction potential and the temperature is slightly different. However, we note that the Lennard-Jones and WCA potentials have the same power in the repulsive part. Furthermore, the shear modulus is known to be only moderately dependent on temperature 239.

Figure 5.8 shows the relaxation times, calculated according to Eq. (5.28), as well as the Maxwell relaxation time (Eq. (5.29) ), as a function of the density. Both measures of relaxation time show the same qualitative trend. In the dilute regime, the relaxation time clearly decreases with an increasing density. The low-density relaxation times are in good agreement with the relaxation time of the exponential mode, shown in Table 5.1. The relaxation times reach a minimum towards the dense regime $(\tau=0.071$ and $\tau_{M}=0.073$ at $\left.\rho=0.68\right)$ and then increase with density in the dense regime, due to the formation of the molasses tail. 


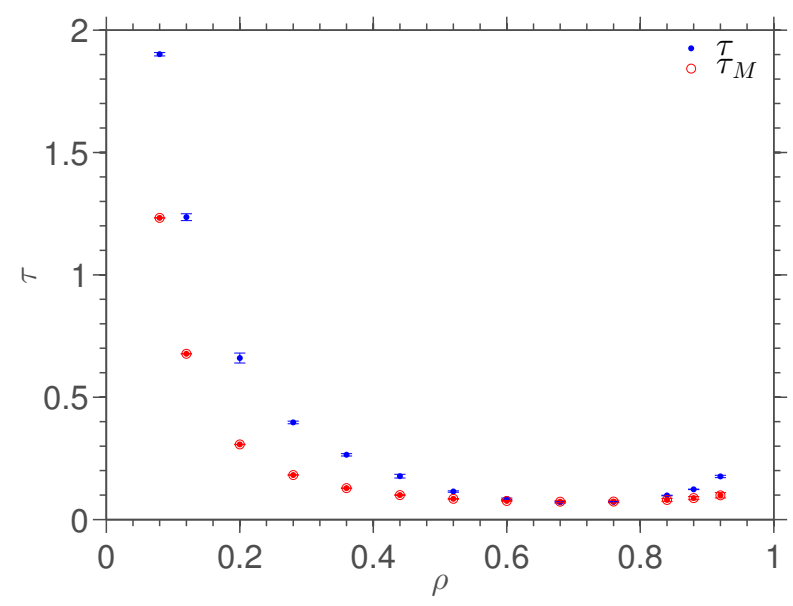

Figure 5.8: Relaxation time (Eq. (5.28) ) as a function of density, compared to the Maxwell relaxation time (Eq. (5.29)).

Figure 5.9 shows the zero-shear rate first normal stress coefficient as a function of the density. The normal stress coefficient and the slope of the function increase towards the dense and the dilute extremes, while a minimum is found around $\rho=0.52$. This remarkable trend was not observed when $\Psi_{1,0}$ was found by extrapolating nonequilibrium simulation data [185].

The larger normal stress coefficients at high densities are related to the memory effect of the viscoelastic fluid. The trend of the zero-shear rate normal stress differences in the dilute regime can be compared with the prediction from kinetic theory, in a similar way as done for the viscosity (Eq. (5.36) )

$$
\Psi_{1,0}(\rho)=\frac{2 p}{\nu^{2}}=\frac{2 \mathrm{k}_{\mathrm{B}} T}{9 \rho A_{2}^{2}} .
$$

This prediction from kinetic theory shows good agreement with our simulation data for densities $\rho \leq 0.52$, as shown in Figure 5.10. The slight over-prediction can be understood since the Maxwell molecules are softer than the WCA atoms. The harder WCA potential leads to a slightly larger collision rate, which results in a lower normal stress coefficient.

The data corresponding to figures 5.6, 5.7, 5.8 and 5.9 are listed in Table 5.2.

\subsection{Non-Newtonian constitutive models}

In the previous section, material constants for a viscoelastic fluid were calculated from equilibrium molecules dynamics (EMD) simulation data. While the viscoelastic 


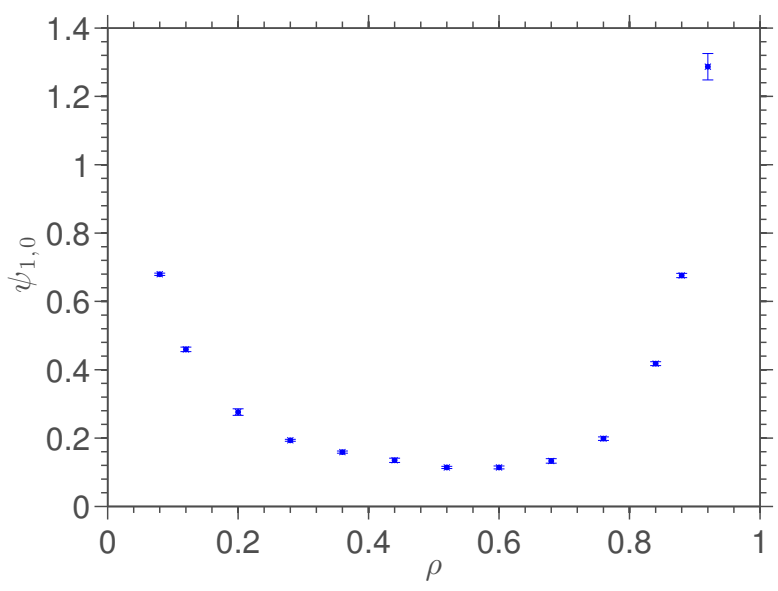

Figure 5.9: First normal stress coefficient as a function of density, obtained from EMD simulations.

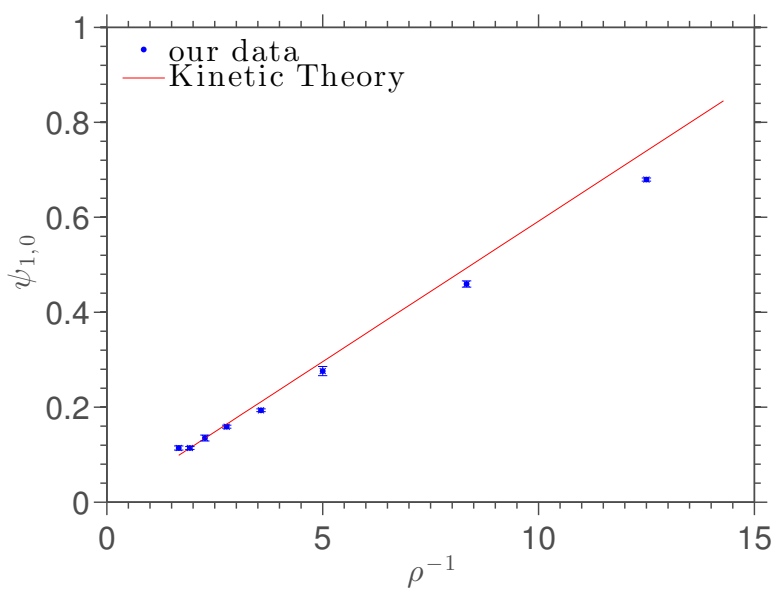

Figure 5.10: First normal stress coefficients of a dilute fluid, compared to kinetic theory. 
Table 5.2: Material properties of a WCA fluid as a function of density and at a fixed temperature $T=1.0$.

\begin{tabular}{cccccc}
$\rho$ & $\eta_{0}$ & $G^{\infty}$ & $\Psi_{1,0}$ & $\tau$ & $\tau_{M}$ \\
\hline 0.08 & $0.1787(3)$ & $0.14493(7)$ & $0.679(3)$ & $1.901(7)$ & $1.2326(4)$ \\
0.12 & $0.1859(6)$ & $0.2745(2)$ & $0.460(7)$ & $1.24(1)$ & $0.6772(7)$ \\
0.2 & $0.2089(9)$ & $0.6809(2)$ & $0.276(9)$ & $0.66(2)$ & $0.3068(9)$ \\
0.28 & $0.2438(6)$ & $1.3397(5)$ & $0.194(3)$ & $0.397(5)$ & $0.1820(8)$ \\
0.36 & $0.3002(6)$ & $2.3414(5)$ & $0.159(3)$ & $0.265(5)$ & $0.1282(7)$ \\
0.44 & $0.381(1)$ & $3.806(1)$ & $0.136(6)$ & $0.177(8)$ & $0.100(2)$ \\
0.52 & $0.4984(9)$ & $5.898(1)$ & $0.114(3)$ & $0.114(3)$ & $0.085(2)$ \\
0.6 & $0.676(1)$ & $8.857(2)$ & $0.114(4)$ & $0.084(3)$ & $0.076(2)$ \\
0.68 & $0.943(2)$ & $12.951(4)$ & $0.133(6)$ & $0.071(3)$ & $0.073(4)$ \\
0.76 & $1.373(2)$ & $18.632(5)$ & $0.198(5)$ & $0.072(2)$ & $0.074(5)$ \\
0.84 & $2.127(3)$ & $26.497(5)$ & $0.418(6)$ & $0.098(1)$ & $0.080(6)$ \\
0.88 & $2.739(2)$ & $31.303(6)$ & $0.676(6)$ & $0.123(1)$ & $0.088(6)$ \\
0.92 & $3.650(8)$ & $36.826(8)$ & $1.29(4)$ & $0.176(5)$ & $0.10(1)$
\end{tabular}

properties in the linear flow regime can be calculated in this way, the non-Newtonian phenomena associated with the nonlinear flow regime were not predicted. In order to study the non-Newtonian behavior, non-equilibrium simulations are required with a deformation rate that is sufficiently large. In this section, a model is presented that predicts the pressure tensor for a non-Newtonian bulk fluid under a homogeneous planar flow field. The model provides a quantitative description of the strain thinning viscosity, pressure dilatancy, deviatoric viscoelastic lagging and out-of-flow-plane pressure anisotropy. Through these four quantities, the non-equilibrium pressure tensor is completely and objectively described and can be calculated as a function of the equilibrium material constants and the velocity gradient. This constitutive framework departs from the conventional Cartesian description of the pressure tensor but regards invariants instead.

Any tensor can be decomposed into an isotropic part and a (traceless) deviatoric part. For an (idealized) incompressible Newtonian fluid, under an arbitrary flow type, we can write

$$
\mathbf{P}_{N}=p_{0} \mathbf{I}-\eta_{0} \mathbf{S}
$$

such that the magnitude of the isotropic equilibrium part is determined by the hydrostatic pressure $p_{0}$ and the deviatoric viscous pressure tensor is given by the product 
of the strain rate tensor $\mathbf{S}$ and the zero-shear viscosity $\eta_{0}$.

The deviatoric pressure tensor can be rewritten in terms of a diagonal (principal) tensor rotated away from its principal orientation to the orientation of the pressure tensor [147, 208, 240. We then obtain the product of a scalar, to represent the magnitude of the deviatoric pressure tensor, and a matrix to denote its orientation. For a Newtonian fluid under any planar flow type with the velocity and its gradient in the $x-y$ plane, Eq. 5.40 can be written as

$$
\begin{aligned}
\mathbf{P}_{N} & =p_{0} \mathbf{I}-\eta_{0} s \mathbf{R}\left(\phi_{S}\right) \cdot \mathbf{I}_{D} \cdot \mathbf{R}^{T}\left(\phi_{S}\right) \\
\mathbf{I}_{D} & \equiv\left(\begin{array}{ccc}
1 & 0 & 0 \\
0 & -1 & 0 \\
0 & 0 & 0
\end{array}\right), \\
\mathbf{R}(\phi) & \equiv\left(\begin{array}{ccc}
\cos \phi & -\sin \phi & 0 \\
\sin \phi & \cos \phi & 0 \\
0 & 0 & 1
\end{array}\right)
\end{aligned}
$$

where $s \equiv \sqrt{\frac{1}{2} \mathbf{S}: \mathbf{S}}$ is the magnitude of the strain rate tensor $(\mathbf{S}: \mathbf{S}$ is the second scalar invariant of $\mathbf{S}), \phi_{S}$ is the angle between the $x$-axis and the eigenvector that corresponds to the maximum eigenvalue of the strain rate tensor, $\mathbf{R}$ is a rotation matrix, that rotates around the $z$-axis (perpendicular to the flow plane) in counter-clockwise direction [241] and $\mathbf{I}_{D}$ is a (traceless) unit deviator matrix. The product of the unit deviator and the scalar pre-factors denotes the principal deviatoric pressure tensor for a Newtonian fluid $\eta_{0} s \mathbf{I}_{D}$. The diagonal elements of this term $\left(\eta_{0} s,-\eta_{0} s, 0\right)$ are the eigenvalues of the deviatoric pressure tensor, while the rotation matrix $\mathbf{R}\left(\phi_{S}\right)$ consists of the corresponding eigenvectors. This notation replaces the usual expression in terms of shear stress and normal stresses by a notation that is not explicitly dependent on the Cartesian tensor orientation, but rather on tensor eigenvalues and the velocity gradient magnitude.

The principal orientation angle of the strain rate tensor for a simple shear flow is $\phi_{S}=45^{\circ}$ and for planar elongational flow it is $\phi_{S}=0^{\circ}$. The orientation angle of the strain rate tensor for a planar mixed flow depends on the ratio between $\dot{\gamma}$ and $\dot{\epsilon}$ and is given by $\phi_{S}=\tan ^{-1}(\dot{\gamma} /(2 \dot{\epsilon})) / 2$. The Newtonian pressure tensor assumes collinearity of the pressure tensor and strain rate tensor. This is often not the case for real fluids, such that $\phi_{P} \neq \phi_{S}$, where $\phi_{P}$ is the angle between the $x$-axis and the eigenvector that corresponds to the principal angle of the deviatoric pressure tensor. The difference between $\phi_{P}$ and $\phi_{S}$ is related to the viscoelasticity of the fluid and will be discussed in more detail below.

Eqs. 5.40 and (5.41) contain the equilibrium values of the shear-viscosity and hydrostatic pressure, whereas real fluids tend to exhibit shear thinning and pressure 
dilatancy when they are deformed sufficiently fast. Thus, this model, in which the viscous pressure tensor is a linear function of the shear rate, does not account for the more complex behavior that some fluids exhibit even at small deformation rates, such as pressure dilatancy and normal stress differences. These non-Newtonian phenomena are generally negligible for a simple fluid at small strain rates, but may become significant for a molecular fluid, even at small strain rates. On the other hand, many existing models that do account for shear thinning and pressure dilatancy are limited to only a specific type of flow. There is currently no simple model that quantifies the pressure tensor for a homogeneous non-Newtonian shear-thinning fluid subject to an arbitrary planar velocity gradient, while some less general relations have been developed for granular fluids under simple shear flow [242, 243] or under isotropic compression [244]. One could attempt to describe the pressure tensor of a viscoelastic fluid simply by replacing $\phi_{S}$ by $\phi_{P}$ in Eq. (5.41), but this is not enough to accurately describe the pressure tensor of a non-Newtonian fluid. A more sophisticated model is needed and is presented in Section 5.3.1.

An existing family of models to describe the pressure tensor of non-Newtonian fluids make use of a nonlinear tensorial approximation as a function of the flow field 245 247]. Second-order fluid models are the simplest of such models that predict nonNewtonian phenomena, such as normal stress differences and shear dilatancy. These models can be employed to predict the pressure tensor of a fluid under any arbitrary flow field. For simple shear, planar elongation or planar mixed flow, the second-order fluid prediction can be compared to NEMD simulation data. One of these models will be briefly discussed here and a comparison is made between the prediction and simulation data for a simple WCA fluid under various planar flow types to assert that the use of a second-order model is not suitable to predict the pressure tensor of a shear-thinning fluid. In this model, the material constants $\eta_{0}, \Psi_{1,0}$ and $\Psi_{2,0}$ (discussed in Section 5.1) are used to quantify the viscous part of the pressure tensor of a non-Newtonian fluid.

The pressure tensor for a second-order fluid can be described by the Rivlin-Ericksen constitutive relation 228

$$
\begin{aligned}
\mathbf{P} & =p_{0} \mathbf{I}-\eta_{0} \mathbf{S}+\frac{\Psi_{1,0}}{2} \mathbf{A}-\left(\Psi_{1,0}+\Psi_{2,0}\right) \mathbf{S}^{2} \\
\mathbf{S} & =\nabla \mathbf{u}+(\nabla \mathbf{u})^{T} \\
\mathbf{A} & \equiv \dot{\mathbf{S}}+\mathbf{S} \cdot \nabla \mathbf{u}+(\nabla \mathbf{u})^{T} \cdot \mathbf{S}
\end{aligned}
$$

where $\mathbf{A}$ is the second Rivlin-Erickson tensor, which is a function of the strain rate tensor and the velocity gradient. Furthermore, $\dot{\mathbf{S}}=\mathbf{0}$ in our case of steady flow denotes the derivative of $\mathbf{S}$ with respect to time 248. The first two terms on the right hand side of Eq. (5.44) equal the Newtonian part (Eq. (5.40), whereas the other two terms 
represent the deviation from Newtonian behavior. The predicted pressure tensor for a fluid under planar mixed flow (PMF) can be derived by inserting the velocity gradient for planar mixed flow (Eq. (3.55) ) into Eqs. (5.45) and (5.46), which results in the following Rivlin-Ericksen tensors

$$
\mathbf{S}=\left[\begin{array}{ccc}
2 \dot{\epsilon} & \dot{\gamma} & 0 \\
\dot{\gamma} & -2 \dot{\epsilon} & 0 \\
0 & 0 & 0
\end{array}\right], \quad \mathbf{A}=\left[\begin{array}{ccc}
4 \dot{\epsilon}^{2}+2 \dot{\gamma}^{2} & -2 \dot{\epsilon} \dot{\gamma} & 0 \\
-2 \dot{\epsilon} \dot{\gamma} & 4 \dot{\epsilon}^{2} & 0 \\
0 & 0 & 0
\end{array}\right]
$$

Substituting these tensors into Eq. 5.44 gives for a steady state flow the following pressure tensor

$$
\begin{aligned}
\mathbf{P} & =\left[\begin{array}{ccc}
P_{x x} & P_{x y} & 0 \\
P_{x y} & P_{y y} & 0 \\
0 & 0 & 0
\end{array}\right], \\
P_{x y} & =-\dot{\gamma}\left(\eta_{0}+\dot{\epsilon} \Psi_{1,0}\right) \\
P_{x x} & =p_{0}-2 \dot{\epsilon} \eta_{0}-2 \dot{\epsilon}^{2} \Psi_{1,0}-\Psi_{2,0}\left(\dot{\gamma}^{2}+4 \dot{\epsilon}^{2}\right), \\
P_{y y} & =p_{0}+2 \dot{\epsilon} \eta_{0}+2 \dot{\epsilon}^{2} \Psi_{1,0}-\left(\Psi_{1,0}+\Psi_{2,0}\right)\left(\dot{\gamma}^{2}+4 \dot{\epsilon}^{2}\right) .
\end{aligned}
$$

The model predicts that the first normal stress difference under a planar mixed flow is given by

$$
\mathcal{N}_{1}=P_{y y}-P_{x x}=-\dot{\gamma}^{2} \Psi_{1,0}+4 \dot{\epsilon} \eta_{0}
$$

Note that the shear stress and first normal stress difference are only dependent on the material constants $\eta_{0}$ and $\Psi_{1,0}$ (see Figure 5.11), and not on $\Psi_{2,0}$. These expressions clearly show deviations from the Newtonian model when the shear rate is non-zero $\dot{\gamma}>0$. The predicted shear stress and first normal stress difference under planar elongational flow are identical to the Newtonian prediction. In the case of shear flow, the shear stress is simply Newtonian, without shear thinning, while normal stress effects are accounted for. Combined stress effects arise for planar mixed flow. The equilibrium material constants that are needed to calculate the model prediction for $P_{x y}$ and $\mathcal{N}_{1}$ are calculated as $\eta_{0}=2.32 \pm 0.01$ and $\Psi_{1,0}=0.69 \pm 0.03$ from time integrals over equilibrium correlation functions, as is described in Section 5.2 .

The simulations are performed in the isokinetic-isochoric ensemble. Such that the fluid density (i.e., the number of atoms $N=512$ and the system volume are fixed) and temperature are kept at a constant value, while the pressure tensor can depend on the state point and on the flow. The fluid density is $\rho=0.8442$ and its temperature $T=0.722$. This state point is near the triple point that a Lennard-Jones fluid with the same parameters would have. This is the most extensively studied state point for MD simulations of a simple atomic fluid such as Argon or Krypton [72. The simulations correspond to combinations of shear rates $\dot{\gamma}=0,0.1, \ldots, 0.5$ and 


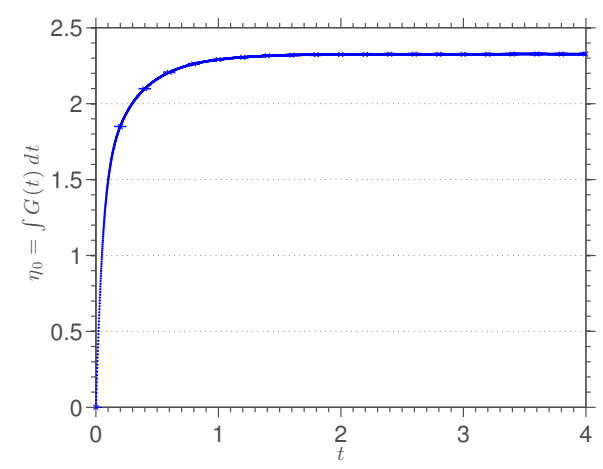

(a)

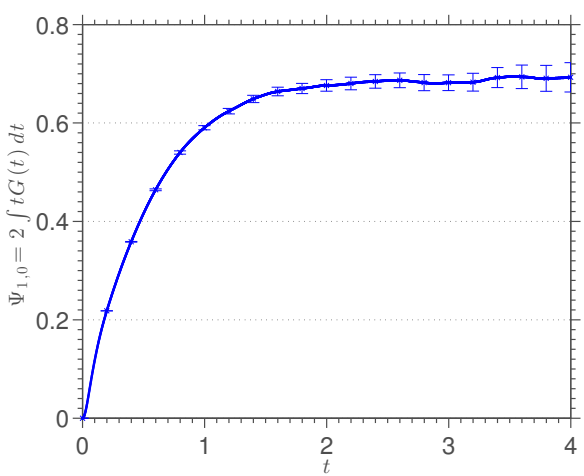

(b)

Figure 5.11: Zero-shear rate viscosity and zero-shear rate first normal stress coefficient $(\rho=0.8442, T=0.722)$.

elongational rates $\dot{\epsilon}=0,0.1, \ldots, 0.5$, such that the combinations of both deformations represent simple shear $(5 \times)$, planar elongation $(5 \times)$ and planar mixed flow $(25 \times)$ simulations. Furthermore, additional shear flow simulations have been performed at shear rates $\dot{\gamma}=0.025,0.05$ and 0.15 . The temperature is controlled using a Gaussian isokinetic thermostat [114. The fluid is first equilibrated to reach a non-equilibrium steady state, followed by a simulation of 500 units of time. The data is averaged over time, and over 10 equivalent simulations with different random initial configurations. The equilibrium material constants are shown in Figure 5.11. These constants can be substituted into the second-order fluid model to predict the non-equilibrium pressure tensor.

Figure 5.12 shows the shear stress $P_{x y}$ and the first normal stress difference $\mathcal{N}_{1}=$ $P_{y y}-P_{x x}$ against the magnitude of the strain rate tensor $s=\sqrt{\frac{1}{2} \mathbf{S}: \mathbf{S}}=\sqrt{\dot{\gamma}^{2}+4 \dot{\epsilon}^{2}}$. Error bars are not shown since the standard errors are smaller than the plotted symbols. The average standard deviation of all data points is shown by the vertical bars directly below the legends. This is a measure for the fluctuations in the respective quantities. The data shows consistent trends with increasing $\dot{\gamma}$ and $\dot{\epsilon}$. The mixed flow data is in agreement with that reported by Hunt et al. [32. Furthermore, the data points on the $x$-axis of Figure $5.12(\mathrm{a})$ correspond to planar elongational flow simulations, whereas the data points close to the $x$-axis of Figure $5.12(\mathrm{~b})$ correspond to shear flow simulations. The data is compared to a second-order fluid model prediction. The lines in Figure 5.12 indicate the trends predicted by the SOF model. The dashed lines correspond to constant $\dot{\epsilon}$, whereas the dash-dotted lines correspond to constant $\dot{\gamma}$. As $\dot{\gamma}$ or $\dot{\epsilon}$ increases, so does the deviation from the SOF model prediction, due to strain 


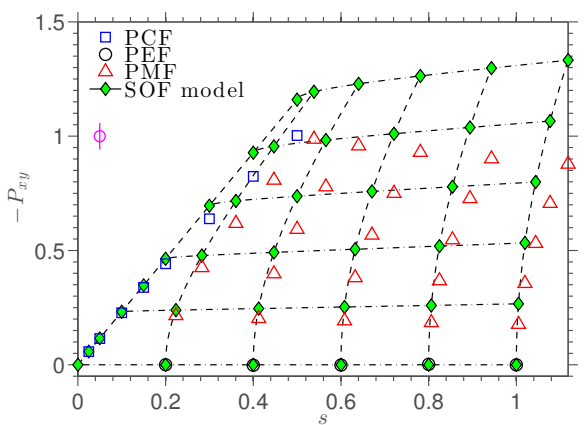

(a)

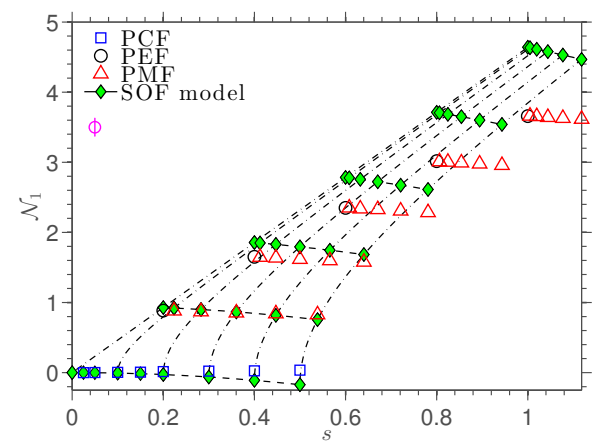

(b)

Figure 5.12: NEMD simulation data of the shear stress (a) and first normal stress difference (b) for a fluid under shear (PCF), elongation (PEF) and planar mixed flow (PMF) compared to the prediction of the second-order fluid (SOF) model $(\rho=0.8442$, $T=0.722)$.

thinning. The inability of the model to accurately predict the pressure tensor in the thinning regime, makes an alternative approach desirable.

A symmetric pressure tensor contains six independent quantities, in general. This reduced to four independent quantities in the case of a steady-state planar flow, where the only non-zero shear stress corresponds to the plane of flow. Therefore, the pressure tensor for a simple atomic fluid under a planar flow can be unique described with a model that contains minimal four parameters.

Rather than looking at the shear stress and first normal stress difference, we can look at objective material properties that are only dependent on the magnitude of the strain rate tensor and not on the type of flow. The thinning behavior that was seen in the shear stress and the normal stress differences in Figure 5.12 is related to the viscosity, which is the proportionality constant between a driving force and the corresponding resulting flux [28]. An expression for the generalized, objective viscosity can be derived from the steady rate of heat production per unit volume, as discussed by Hounkonnou et al. 207.

$$
\eta(\dot{\gamma}, \dot{\epsilon})=-\frac{\mathbf{P}: \mathbf{S}}{\mathbf{S}: \mathbf{S}}=\frac{-\dot{\gamma} P_{x y}+\dot{\epsilon} \mathcal{N}_{1}}{\dot{\gamma}^{2}+4 \dot{\epsilon}^{2}} .
$$

The viscosity computed with this expression is independent of the choice of the coordinates in the flow plane, and thus objective, as the double tensor contractions in the numerator and denominator of the expression imply. When the elongational rate is zero, Eq. 5.50 simplifies to the well known expression for shear $\eta=-P_{x y} / \dot{\gamma}$, 


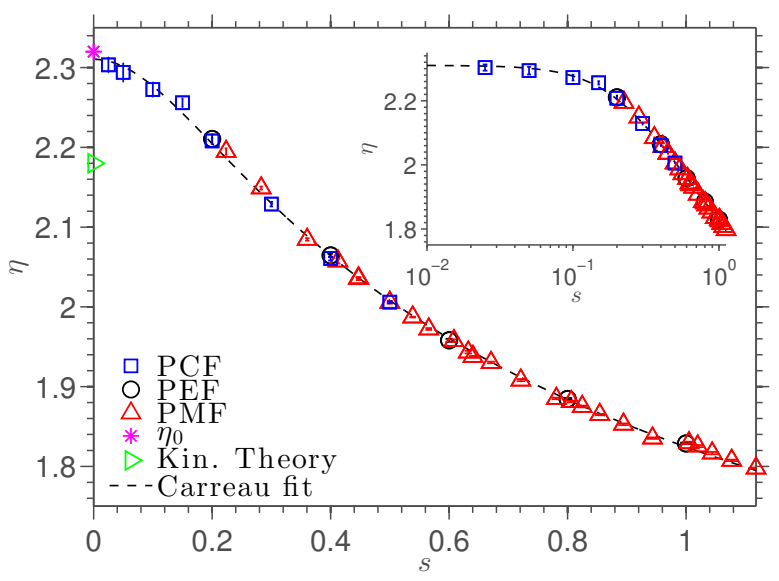

Figure 5.13: Viscosity of a WCA fluid under shear (PCF), elongation (PEF) and mixed flow (PMF) at state point $\rho=0.8442, T=0.722$. The data is fitted with a Carreau model and the equilibrium viscosity and a kinetic theory prediction are shown at $s=0$.

whereas a situation in which the shear rate is zero results in the elongational viscosity $\eta=\mathcal{N}_{1} /(4 \dot{\epsilon})$.

Figure 5.13 shows the viscosity data of a WCA fluid under shear, elongation and mixed flows. The viscosity approaches the Newtonian viscosity $\eta_{0}$ in the equilibrium $(s \rightarrow 0)$ limit and shows a monotonic decay with the strain rate magnitude. The fact that all data points in Figure 5.13 collapse onto a profile that is a function of the strain rate magnitude $s$ only, implies that the viscosity of the WCA fluid is independent of the flow type. It does however depend on the strength of the deformation rate, which is proportional to the square root of the rate of energy dissipation. The data is fitted with a Carreau function [249] as $\eta=\eta_{0} /\left(1+c_{\eta 1} s^{2}\right)^{c_{\eta 2}}$, where $\eta_{0}=2.32 \pm 0.01$ is the zero-shear rate viscosity, $c_{\eta 1}=21.21$ and $c_{\eta 2}=0.076$. The positive power $c_{\eta 2}$ indicates that the fluid is shear thinning, whereas a negative number would correspond to a shear thickening fluid. The zero-shear rate viscosity $\eta_{0}$ can be compared to a kinetic theory prediction for a dense fluid of inelastic spheres 250. We calculate an effective volume fraction $\nu=0.4674$ by substituting our density, temperature and zeroshear rate viscosity into Eq. (2) from Ref. 250]. Using the effective volume fraction, temperature and equilibrium pressure of the WCA fluid, the kinetic theory model predicts a zero-shear rate viscosity of $\eta^{K T}=2.18$, which is $6.2 \%$ smaller than our simulation result. 


\subsubsection{Rotating the pressure tensor}

We start our derivation by subtracting the first tensor invariant, i.e., the pressure $p=\operatorname{tr}(\mathbf{P}) / 3$, from the diagonal of the pressure. What remains is the (traceless) deviatoric pressure tensor $\mathbf{P}^{D}=\mathbf{P}-p \mathbf{I}$. Furthermore, the deviatoric pressure tensor is rotated to its principal (shear-free) orientation. The principal orientation of the pressure tensor, in a planar stress situation, is defined by a single angle $\phi_{P}$ that denotes the direction of one of the eigenvectors in the plane of flow. The correctness of this simplification, i.e., the fact that one eigendirection is perpendicular to the plane of flow, has been confirmed by the simulation data.

The Newtonian model in Eq. 5.41) expressed the pressure tensor as a function of $s$ in terms of the principal orientation angle of the strain rate tensor $\phi_{S}$ and equilibrium material constants $\eta_{0}=2.32 \pm 0.01$ and $p_{0}=6.3903 \pm 0.0002$. The material constants for a non-Newtonian fluid are not simply dependent only on the type of flow and the state point of the fluid. Non-equilibrium values of $\eta$ and $p$ tend to deviate from their equilibrium values due to shear thinning and pressure dilatancy, respectively, and collinearity between the pressure tensor and strain rate tensor may no longer be assumed for a viscoelastic fluid. Thus, the model parameters $\left(\eta, p, \phi_{P}\right)$ are not by definition equal to their equilibrium values that were used in the Newtonian model. Furthermore, an additional parameter may be required to uniquely describe the four independent non-zero components of the pressure tensor for a fluid under a planar flow type. We will first express the deviatoric pressure tensor in terms of its eigenvalues and its principal orientation (which is related to its eigenvectors) and then we will study the relation to the strain rate tensor.

The constitutive relation in Eq. 5.41 can be generalized for a non-Newtonian fluid. Doing so, we have to take into account that: (1) the principal orientation of the deviatoric pressure tensor is not by definition identical to that of the strain rate tensor, (2) we do not know the eigenvalues of the deviatoric pressure tensor, hence we also cannot directly split the principal deviatoric pressure tensor in a scalar magnitude and a unit deviator matrix, as was done in Eq. 5.41. We can write the pressure tensor as

$$
\mathbf{P}=p \mathbf{I}+\mathbf{R}\left(\phi_{P}\right) \cdot\left(\begin{array}{ccc}
-\lambda_{1}-\lambda_{2} & 0 & 0 \\
0 & \lambda_{1} & 0 \\
0 & 0 & \lambda_{2}
\end{array}\right) \cdot \mathbf{R}^{T}\left(\phi_{P}\right),
$$

where the eigenvalues of the deviatoric pressure tensor are arranged as $\lambda_{1} \geq \lambda_{2} \geq$ $\lambda_{3}=-\lambda_{1}-\lambda_{2}$. The order in subscripts is convention, while the order in magnitude of eigenvalues can be understood by thinking of planar elongational flow, in which the principal pressure tensor equals the pressure tensor, such that the diagonal elements of the deviatoric pressure tensor are equal to its eigenvalues. The stretch in the $x$ direction results in the smallest diagonal component of the pressure tensor, while the 
contraction in the $y$-direction corresponds to the largest diagonal component of the pressure tensor, and the out-of-flow-plane direction has an intermediate value.

In summary, the model expresses the pressure tensor in terms of non-equilibrium pressure $p=p_{0}+\Delta p$, the orientation angle of the principal deviatoric pressure tensor $\phi_{P}$ and two independent eigenvalues of the deviatoric pressure tensor $\lambda_{1}$ and $\lambda_{2}$. For further understanding, we study the dependence of these quantities on the velocity gradient.

\subsubsection{An objective model to describe and predict the non- Newtonian pressure tensor}

Figure 5.14 shows the pressure and the eigenvalues of the deviatoric pressure tensor as functions of the magnitude of the strain rate tensor. Both graphs show clear trends with $s$ and no visible dependency on the flow type. The pressure in Figure 5.14(a) increases with increasing $s$. This so-called pressure dilatancy is proportional to $\Delta p \propto$ $s^{3 / 2}$ over almost two orders of magnitude, as shown in the inset. The error bars in the inset denote the standard deviation of the data points. This proportionality has been reported in the literature [251, 252] for a Lennard-Jones fluid at the same state point and comparable deformation rates, while Lennard-Jones data for other state points suggested different proportionalities. The scaling with the deformation rate is different for very small $s$. A proportionality $\Delta p \propto s^{2}$ has been observed for a WCA fluid near the LJ triple point, sheared at small rates $10^{-8}<\dot{\gamma}<10^{-2}$, using the transient-time correlation function (see Chapter60 252. The pressure in Figure 5.14(a) is fitted to a function of the form $p=p_{0}+c_{p} s^{3 / 2}$, where $p_{0}$ is the equilibrium pressure and $c_{p}=0.7492$. The eigenvalues of the deviatoric pressure tensor (Figure 5.14(b) would be $\left(\lambda_{1}=\eta_{0} s, \lambda_{2}=0, \lambda_{3}=-\lambda_{1}-\lambda_{2}=-\eta_{0} s\right)$ for a Newtonian fluid. This prediction is shown in the figure with the dash-dotted lines. To account for non-Newtonian phenomena in the plane of flow, an approximation can be made as $(X s, 0,-X s)$, where the middle eigenvalue corresponds to the direction perpendicular to the plane of flow. Substituting Eq. (5.51) into Eq. 5.50) gives

$$
\eta=\frac{2 \lambda_{1}+\lambda_{2}}{2 s} \cos (2 \Delta \phi)
$$

where $\Delta \phi \equiv \phi_{S}-\phi_{P}$ represents the lagging of the pressure tensor relative to the deformation field. The terms $\cos (2 \Delta \phi)$ is close to unity for all the data shown here, but this is not the case for example for a dilute fluid, where a longer relaxation time leads to a larger lagging angle $5^{5}$ This expression is exactly valid for any steady planar

\footnotetext{
${ }^{5}$ This is corroborated by the simulation results that are shown in Appendix $\mathrm{B}$
} 


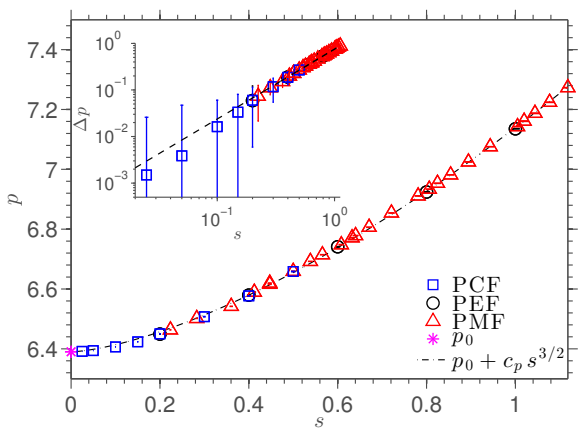

(a)

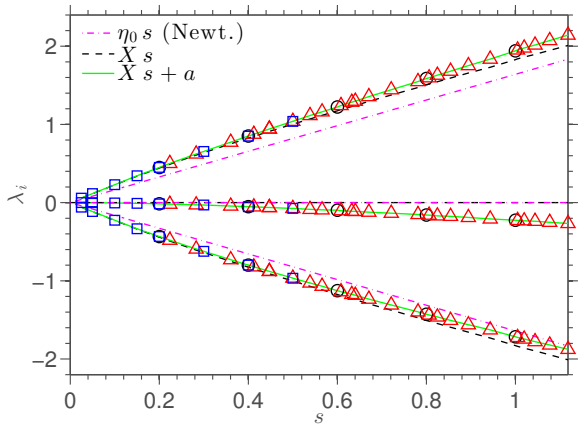

(b)

Figure 5.14: Pressure (a) and eigenvalues of the deviatoric pressure tensor (b) as a function of the magnitude of the strain rate tensor $(\rho=0.8442, T=0.722)$. The inset in (a) gives the proportionality between $p$ and $s^{3 / 2}$ in a logarithmic graph.

flow. If we substitute $\lambda_{1}=X s$ and $\lambda_{2}=0$ into Eq. (5.52), we obtain

$$
X=\frac{\eta}{\cos (2 \Delta \phi)}
$$

This result is shown by the dashed lines in Figure 5.14(b) However, this approximation still assumes a zero intermediate eigenvalue, which is inconsistent with the simulation data. The data shows that the magnitude of the discrepancy is a function of $s$ only. A correction term can be added to the functional form of the eigenvalues as $(X s+$ $a,-2 a,-X s+a)$, such that Eq. (5.52) remains satisfied. The correction term is a function of $s$ (quantified below). A non-zero value for $a$ indicated an out-of-flow-plane anisotropy. The functional form with the correction term leads to perfect agreement with the simulation data for each of the eigenvalues, as shown by the solid lines in the figure 6

Substituting the found functional form for the eigenvalues $\lambda_{1}=\eta s+a$ and $\lambda_{2}=$ $-2 a$ into Eq. 5.51) gives

$$
\mathbf{P}=p \mathbf{I}+\mathbf{R}\left(\phi_{P}\right) \cdot\left(-\eta s \mathbf{I}_{D}+a\left(\begin{array}{ccc}
1 & 0 & 0 \\
0 & 1 & 0 \\
0 & 0 & -2
\end{array}\right)\right) \cdot \mathbf{R}^{T}\left(\phi_{P}\right) .
$$

Since $\lambda_{2} \neq 0$, the unit deviator matrix $\mathbf{I}_{D}$ is not sufficient for the description of the deviatoric pressure tensor and we have gained an additional tensorial term. This

\footnotetext{
${ }^{6}$ The predictive quality of our equations below (Eqs. 5.58, 5.59) and 5.60) that enter the fit that is represented by our green solid lines is within $0.5 \%$ of the simulation data.
} 
term is again written as the product of a scalar magnitude and a traceless matrix. The first term gives a planar, but non-colinear, deviatoric pressure tensor, while the second term quantifies the non-planar anisotropy. Both terms are traceless, and thus deviatoric, but the second is invariant under rotation in the plane of flow. It must be noted that the deviatoric pressure tensor is closely related to the in-plane $\eta_{-}$and out-of-plane $\eta_{*}$ viscosity coefficients [200, 201] that can be used as a measure of nonNewtonian behavior under shear flow. For simple shear flow, these coefficients are defined as $\eta_{-} \equiv \mathcal{N}_{1} /(2 \dot{\gamma})=\left(P_{y y}-P_{x x}\right) /(2 \dot{\gamma})$ and $\eta_{*} \equiv\left(P_{y y}-2 P_{z z}+P_{x x}\right) /(4 \dot{\gamma})$, where the occurrences of the normal pressure terms in the coefficients agree exactly with the diagonals of the planar and the non-planar terms in Eq. 5.54), respectively. The out-of-plane viscosity coefficient $\eta_{*}$ is related to our out-of-plane anisotropy via $\eta_{*}=6 a /(4 s)$, for any type of planar flow.

The tensor rotation on the right hand side of Eq. (5.54) can be split into a rotation about an angle $\phi_{S}$ and an additional rotation by the (negative) lagging angle $-\Delta \phi=$ $\phi_{P}-\phi_{S}$ to obtain the orientation of the pressure tensor $\phi_{P}$. We know from Eqs. 5.40 and 5.41) that $\mathbf{S}=s \mathbf{R}\left(\phi_{S}\right) \cdot \mathbf{I}_{D} \cdot \mathbf{R}^{T}\left(\phi_{S}\right)$, such that the pressure tensor can be written as

$$
\begin{aligned}
\mathbf{P} & =p \mathbf{I}-\frac{\eta}{\cos (2 \Delta \phi)} \mathbf{R}(-\Delta \phi) \cdot \mathbf{S} \cdot \mathbf{R}^{T}(-\Delta \phi)+\sqrt{3} a \mathbf{I}_{a}, \\
\mathbf{I}_{a} & \equiv \frac{1}{\sqrt{3}}\left(\begin{array}{ccc}
1 & 0 & 0 \\
0 & 1 & 0 \\
0 & 0 & -2
\end{array}\right),
\end{aligned}
$$

where $\mathbf{I}_{a}$ is scaled such that the tensor has a scalar magnitude of 1 , exactly like $\mathbf{I}_{D}$. Furthermore, $\mathbf{I}_{a}$ is invariant to rotations in the plane of flow. This model contains the four parameters $p, \eta, \Delta \phi$ and $a$, each is a function of the velocity gradient. The last term in the model is invariant under rotation around the $z$-axis and has an equal contribution in the $x$ and $y$ directions.

The parameter $a$ in our model can be fitted against the magnitude of the strain rate tensor as $a=c_{a} s^{3 / 2}$, with $c_{a}=0.1113$. The data for $a$ and the corresponding fit are shown in Figure 5.15. The fit deviates slightly from the data at small values of $s$. This result indicates that $a$ is proportional to $p-p_{0}$. The inset in the figure shows the out-of-flow-plane anisotropy, normalized by the pressure dilatancy, $a /\left(p-p_{0}\right)$, which approaches a constant value at large $s$. While the out-of-flow-plane anisotropy tends to zero for $s \rightarrow 0$, the ratio between $a$ and $p-p_{0}$ diverges close to $s=0$, since the denominator tends to zero faster than the numerator. The divergence turns out to be strongly related to the discrepancy at small $s$ between the data for $a$ and the fit of the data and is thus not considered to be physically meaningful. The presented model makes it possible to predict the pressure tensor for an atomic fluid, under an arbitrary 


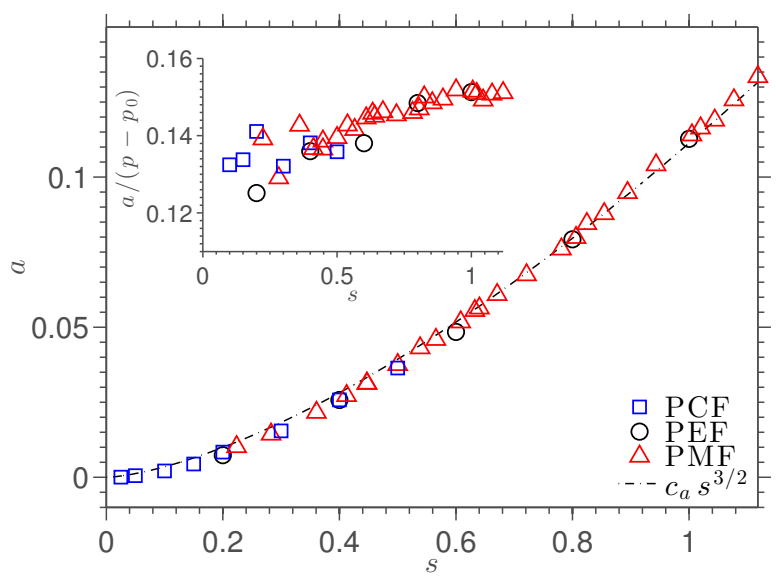

Figure 5.15: The parameter $a$ as a function of the magnitude of the strain rate tensor ( $\rho=0.8442, T=0.722)$. The inset shows the normalized anisotropy.

planar flow field. The parameters $p, \eta$ and $a$ are independent of the flow type and only depend on the state point of the fluid and on $s$, while $\Delta \phi$ is studied in more detail next.

Figure 5.16 shows the orientation angles of the strain rate tensor $\phi_{S}$, the pressure tensor $\phi_{P}$ and the difference (lag) between both angles $\Delta \phi$, given in degrees. The lag angle is very small relative to the orientation angles, which makes it hard to measure with high accuracy. Figure 5.16(b) shows that the lag angle divided by the magnitude of the vorticity $\omega=\dot{\gamma} / 2$ collapses onto a single profile. Note that for small $\omega$ (and many of the data points with small values of $s$ ) the inaccuracy in the ratio $\Delta \phi / \omega$ becomes large. The profile is inversely proportional to $s+b$, where $b$ is a constant that is inversely proportional to a time scale of the fluid in equilibrium, and thus related to the state point of the fluid.

Evans et al. 52] suggested that the distortion of the microstructure of the fluid out of equilibrium should be proportional to a phenomenological relaxation time of the fluid. Similarly to the non-equilibrium structure of the fluid, also the viscoelastic lagging should be proportional to the relaxation time, such that we can write $\Delta \phi=$ $c_{\phi} \omega /(s+b)=\tau \omega$, with $\tau \propto c_{\phi} /(s+b)=1 /\left(\alpha \tau_{s}^{-1}+\tau_{0}^{-1}\right)$, where $\tau_{0}=c_{\phi} / b$ and $\tau_{s}$ are competing equilibrium and non-equilibrium time scales and $\alpha$ is a proportionality constant. Different relaxation times can be defined and calculated. For a fluid at equilibrium, for example, $\tau_{1} \equiv \Psi_{1,0} /\left(2 \eta_{0}\right)=0.69 /(2 \times 2.32)=0.15$ was defined (see Section 5.1.2. Many other equilibrium and non-equilibrium relaxation times can be defined, for example related to the transient response of the pressure tensor [224], 


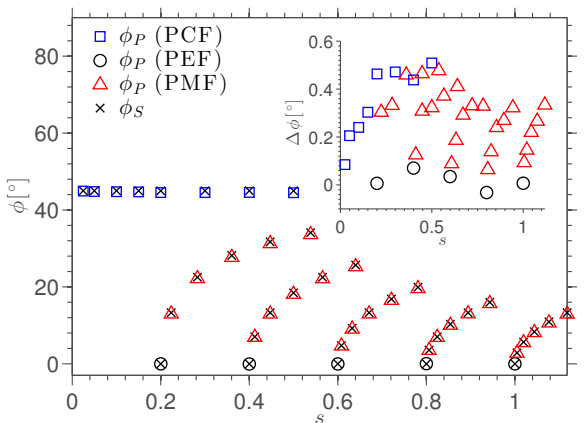

(a)

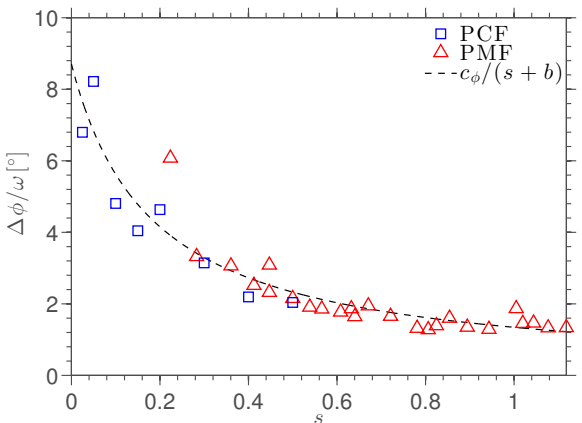

(b)

Figure 5.16: Principal orientation angles (a) as a function of the magnitude of the strain rate tensor $(\rho=0.8442, T=0.722)$, the inset shows the lag angle. In (b), the lag angle is scaled by the vorticity $\omega$, such that the data collapses onto a single profile.

distortion of the pair distribution function [193], or to the collision frequency [253].

In summary, the pressure tensor for a WCA fluid near the LJ triple point and under an arbitrary planar flow field in the thinning regime can be expressed in terms of four variables

$$
\begin{aligned}
\Delta p & =c_{p} s^{3 / 2}, \\
\eta & =\eta_{0} /\left(1+c_{\eta 1} s^{2}\right)^{c_{\eta 2}}, \\
a & =c_{a} s^{3 / 2}, \\
\Delta \phi & =c_{\phi} \omega /(s+b),
\end{aligned}
$$

where each quantity represents a deviation from Newtonian behavior, i.e., pressure dilatancy, strain thinning, non-planar pressure tensor anisotropy and viscoelastic lagging. Substituting these parameters into Eq. 5.55) completes the presented model. With this model, the pressure tensor can be predicted for a WCA fluid near the LJ triple point for any given planar velocity gradient. If the equilibrium fluid properties are known, the model needs only six fitting parameters. Alternatively, the shear viscosity and pressure in the zero-shear rate limiting case can be included as fitting parameter in the model, which brings the total number of parameters to eight. This has been tried as well, the fitted values for $\eta_{0}$ and $p_{0}$ were within the statistical uncertainty of our values from EMD simulations.

In order to test the limits and predictive value of our model, we have performed simulations of a fluid under a larger deformation rate than that of the previous simulations. The model is tested for a fluid under a planar elongational flow with $\dot{\epsilon}=1.0$, 
such that $s=2$. The orientation angle of the pressure tensor should be zero for planar elongational flow, the simulation result gives $\phi_{P}=0.0160$, with a large relative uncertainty. The agreement between the model and the simulation results for pressure, viscosity and anisotropy are $-0.6 \%,-3.0 \%$ and $+0.8 \%$, respectively. This shows that the model predicts the pressure tensor with high accuracy even at larger deformation rates. We increase the deformation rate a bit further and test the model for a fluid under planar mixed flow with $\dot{\gamma}=\dot{\epsilon}=1.0$, corresponding to $s=\sqrt{5}$ and $\phi_{S}=13.28^{\circ}$. The predicted values for $p, \eta, a$ and $\Delta \phi_{P}$ are all within $4 \%$ of the simulation results.

We have also performed simulations for $\dot{\gamma}=\dot{\epsilon}=0.01\left(s=\sqrt{5 \times 10^{-4}}\right)$ to verify the convergence towards Newtonian behavior near equilibrium. We have measured $\eta=2.33 \pm 0.02$ and $p=6.3897 \pm 0.0004$, which means that both quantities agree within the standard error with the values calculated with EMD simulations. Deviations from the Newtonian limiting behavior are too small to be measured accurately from NEMD simulations very close to equilibrium unless significantly more statistics are accumulated, or alternative techniques are used to enhance the statistics [254].

\subsection{Transient flows}

A simple relation between the instantaneous shear stress and the deformation of a viscoelastic fluid was shown in Section 5.1.2. This theory predicted the shear stress response as a linear function of the deformation rate. The predicted instantaneous shear stress can be compared to values calculated from NEMD simulations. Describing the instantaneous stresses is especially important for processes in which the deformation rate is not constant in time, or processes that have a start-up and an end (this is for example the case in pumps).

Figure 5.17 shows a simple example of three flow situations, each with their own typical behavior and interesting quantities to study. The data in this figure is averaged over 10 independent simulations, each with 108,000 WCA particles at the phase point $\rho=0.8442$ and $T=0.722$, sheared at a shear rate $\dot{\gamma}=0.5$. In the first two flow situations, i.e., startup flow and steady state flow, the fluid is homogeneously sheared at a constant shear rate $\dot{\gamma}=0.5$, whereas the relaxation flow corresponds to a situation in which a constant shear rate on a fluid is suddenly removed. In a startup or cessation flow, the fluid is in transition from one steady state to another. In many cases, one of the steady states corresponds to equilibrium. In this section, we study components of the pressure tensor and the shear viscosity profile in startup flows from equilibrium to a non-equilibrium steady state and in cessation flows from a non-equilibrium steady state to equilibrium.

These two types of transient flows both display a lagging between the external field 


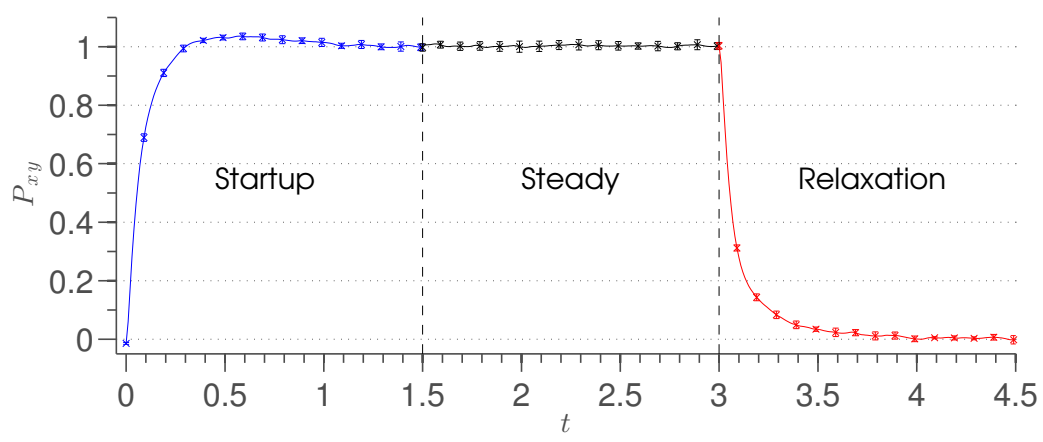

Figure 5.17: Three flow situations are shown: startup flow, steady state flow and relaxation to equilibrium.

and the response of the viscoelastic fluid, as discussed in Section 5.1. Figure 5.17 shows that the startup profile can exhibit an overshoot in shear stress before converging to the steady-state value, whereas the relaxation of shear stress from a non-equilibrium steady state shows no overshoot and shows a profile that resembles an exponentially decaying function.

Picu and Weiner 224] showed that even a dense simple atomic liquid relaxes to equilibrium with two different relaxation modes. Nonlinear stress response can complicate the picture even more, as is the case for large deformation rates [28] and for a molecular fluid 255]. The linear shear stress response can be exactly predicted using the zero-shear rate viscosity, that can be calculated in equilibrium using the GreenKubo relation. However, many interesting phenomena are related to deviation from the linear constitutive relations. Alternatively, NEMD simulations give the exact transient response, regardless of the rate of deformation. Furthermore, the transient-time correlation function, a nonlinear generalization of the Green-Kubo relations, can be used to calculate the transient stresses of a fluid. This method will be treated in more detail in Chapter6.

\subsubsection{Startup flow}

In this section, we briefly look at the shear stresses in a sheared simple atomic fluid in a startup flow. The term 'startup flow' refers to a situation in which a constant deformation rate is superimposed on a fluid in equilibrium at time $t=0$. This constant deformation rate drives the fluid to a non-equilibrium steady state. The transient stresses and the time it takes to reach the steady state can depend on the deformation rate, the type of fluid and its state point and should not depend on simulations details, 
such as the type of integrator or the thermostat. However, care must be taken in performing such simulations correctly. For example, many integration schemes need a number of time steps to settle, i.e., they are not self-starting. Calculation of transient properties always requires a self-starting algorithm, such as a Runge-Kutta scheme (see Section 2.1. Furthermore, a profile-biased thermostat becomes unsuitable if the shear rate is very large (the critical shear rate depends on the state point and the thermostat, near the LJ triple point we started observing the string phase for $\dot{\gamma} \approx 3.0$ ), as discussed in Section 2.5.

Figure 5.18 shows the transient shear stress and shear viscosity of a WCA fluid under a steady shear rate. The stresses, and corresponding viscosity profiles, measured from the NEMD simulation are compared to the predicted linear response given by the appropriate Green-Kubo (GK) integral (see Section 4.4). The shear stress profiles in Figure 5.18(a) correspond, from top to bottom, to shear rates from $\dot{\gamma}=0.1$ to $\dot{\gamma}=1.0$, with increments of $\Delta \dot{\gamma}=0.1$. If the stress response is a linear function of the shear rate, it would overlap with the GK prediction. The figure shows that only the data corresponding to the smallest shear rate shows good agreement with the GK profile. The deviation from this linear stress response increases with an increasing shear rate. Furthermore, the stress profiles that corresponds to a shear rate $\dot{\gamma} \geq 0.4$ show an overshoot before converging to its steady-state value. Heyes et al [256] were the first to observe this overshoot for a simple fluid and suggested a possible mechanism for its origin. They explained that if a fluid is sheared, structural rearrangements are responsible for a shear thinning viscosity. The amount of structural rearrangement that is required at high shear rates, take longer to develop than the viscoelastic relaxation. Such that the shear stress first increases to a maximum value, followed by a decrease in shear stress due to structural changes in the fluid. The difference between the actual shear stress and the Green-Kubo prediction is shown in Figure 5.18(b). The structural rearrangement and the related shear thinning starts around the same time for all shear rates. While the amount of rearrangement that is required increases with the shear rate, the time it takes to reach a configuration with the least shear resistance does not show an increase with the shear rate. Figure 5.18(c) shows the shear stress divided by respective shear rate, i.e., the shear viscosity $\eta=-P_{x y} / \dot{\gamma}$. At very small deformation rates $(\dot{\gamma} \leq 0.025$ (Figure 5.13) ) the fluid behaves Newtonian. If this is the case, the viscosity profile shows no dependence on the shear rate and overlap with the equilibrium shear viscosity $\eta_{0}$, predicted by the GK integral for shear viscosity. As the shear rate increases, shear thinning occurs and the shear viscosity decreases with an increasing shear rate. The data for the shear viscosity in Figure 5.18(c) becomes more noisy as the shear rate decreases, i.e., .the signal-to-noise ratio becomes low, where the signal refers to the measured shear stress. The lowest shear rate considered here is still 
many orders of magnitudes larger than those relevant for many industrial processes or shear rates measurable in experiments. This signifies the importance of a method that can calculate the fluid properties under small deformation fields. The transient-time correlation function, that was introduced in Section 4.5, is such a method needed. This method will be applied to several flow problems in Chapter6.

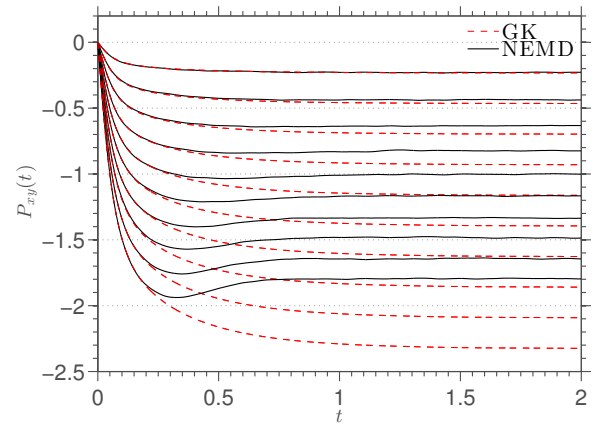

(a)

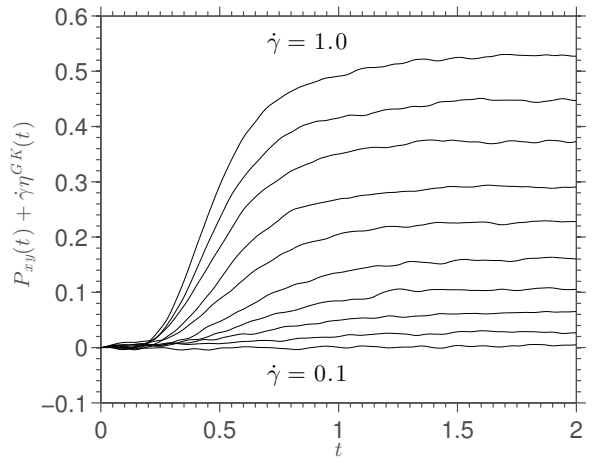

(b)

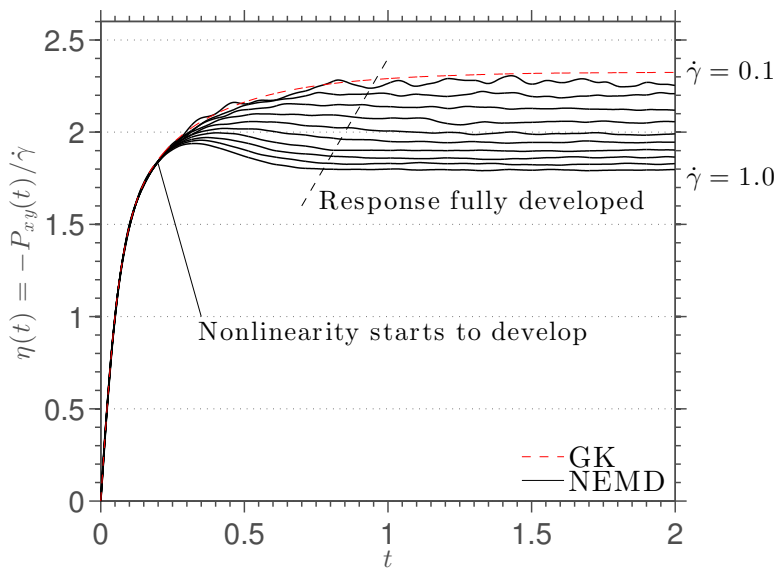

(c)

Figure 5.18: Transient shear stress and shear viscosity in shear flow $(\rho=0.8442$, $T=0.722)$.

Figure 5.19 shows direct NEMD averages of the transient viscosity after the activation of various constant external fields. The fluid consists of WCA particles at a reduced density $\rho=0.8442$ and a temperature $T=0.722$. The fields are as follows: PCF at $\dot{\gamma}=1.0, \mathrm{PEF}$ at $\dot{\epsilon}=0.5$ and PMF at $\dot{\gamma}=0.6$ and $\dot{\epsilon}=0.4$. These external fields 


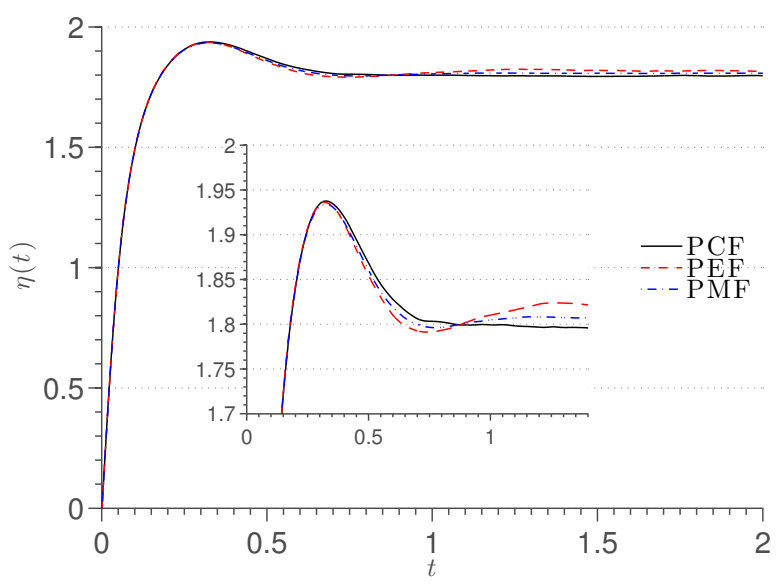

Figure 5.19: Direct NEMD averages of the transient viscosity after the activation of various constant external fields. The fluid consists of WCA particles at a reduced density $\rho=0.8442$ and a temperature $T=0.722$. The fields are as follows: PCF at $\dot{\gamma}=1.0, \mathrm{PEF}$ at $\dot{\epsilon}=0.5$ and PMF at $\dot{\gamma}=0.6$ and $\dot{\epsilon}=0.4$. These external fields have the same second scalar invariants, such that they are comparable. This is consistent with the observation from the steady-state simulations in Section 5.3 .

have the same second scalar invariants $\mathrm{I}_{2}=\mathbf{S}: \mathbf{S}=2 \dot{\gamma}^{2}+8 \dot{\epsilon}^{2}=2.0$. Note that there is an infinite number of combinations of shear and elongation that have the same scalar invariant. However, the shear viscosity of a simple atomic fluid is not dependent on the ratio between shear and elongation, as also shown for steady state flows in Section 5.3 . Also the transient profiles in Figure 5.19 show no clear dependence on the flow type, the profiles are within $1 \%$ of each other.

\subsubsection{Relaxation to equilibrium}

In addition to the EMD approach presented in Section 5.1, the stress relaxation time can also be directly measured from non-equilibrium simulations in which the fluid relaxes from a non-equilibrium steady state after the driving field is switched off. Gao and Weiner [257] observed that the relaxation of a simple fluid could be described using only 2 exponential modes. The authors performed their simulations without using a strictly homogeneous algorithm, such as the SLLOD equations of motion [26, 27]. However, their system is stiff to ensure that the propagation of information is fast, resulting in only a small inaccuracy in the transient response. Picu and Weiner [224] showed, for a simple fluid under planar elongational flow, that the first relaxation mode 
corresponds to the distribution of nearest neighbors, while the second mode is related to the relaxation of anisotropy of the number density distribution.

Linear viscoelastic theory can be used to predict the response of shear stress after sudden cessation of steady state shear flow using Eq. (5.19). Note that this expression applies only in the range of linear stress responses, such as the shear-stress response to a small shear rate or the normal stress response in the case of elongational flows or combinations of shear and elongational flows with small deformation rates. Normal stress differences in shear flow, however, are a nonlinear effect and thus not captured by this linear rheological equation of state.

We have already shown that at moderate density, the stress relaxation function is well described by a Gaussian plus two exponentials. This can be integrated to find the stress response after the cessation of steady shear flow using Eq. 5.19. A similar approach is used by Picu and Weiner 224] to approximate the stress relaxation of a viscoelastic fluid

$$
P_{\alpha \beta}(t)=\sum_{i} C_{i} \mathrm{e}^{-\frac{t}{\tau_{i}}}
$$

where each stress component $P_{\alpha \beta}$ can have different proportionality constants $C_{i}$ and relaxation times $\tau_{i}$. The shear stress relaxation of a simple fluid can be approximated with two modes 257]. Thus, 4 unknowns $\left(C_{1}, \tau_{1}, C_{2}, \tau_{2}\right)$ have to be found to fit the simulation data to a function of the form of Eq. 5.61). We can reduce the number of fitting parameters for the shear stress by substituting $C_{2}=P_{x y}(0)-C_{1}$. Taking $P_{x y}(0)$ in front of the equation and dropping the subscript of the constant, reads

$$
P_{x y}(t)=P_{x y}(0)\left(C \mathrm{e}^{-\frac{t}{\tau_{1}}}+(1-C) \mathrm{e}^{-\frac{t}{\tau_{2}}}\right) .
$$

The constant $C$ represents the initial weight of the first mode relative to the second.

Our relaxation simulations are performed at a density $\rho=0.84$ and temperature $T=1.0$. The fluid is sheared at a constant rate until a steady state is reached, after which point the driving field is removed suddenly. The non-equilibrium simulations contains 512 atoms and the data is obtained from an average over $12 \times 10^{4}$ trajectories. To maintain a constant temperature, the generated heat needs to be removed from the system. A kinetic thermostat assumes a knowledge of the velocity profile. This knowledge is not available when the fluid is not in a steady-state. Instead, a configurational thermostat [129, 131] is used to control the temperature during the relaxation to equilibrium.

In Figure 5.20 the relaxation after a sudden cessation of steady shear flow for $\dot{\gamma}=0.1$ is shown for a dense liquid at $\rho=0.84$ and temperature $T=1.0$. The relaxation of shear stress obtained from a NEMD simulation is in good agreement with the profile predicted from linear viscoelastic theory. This confirms that the linear viscoelastic theory, presented in Eq. 5.19, is satisfied. The shear stress is also predicted 


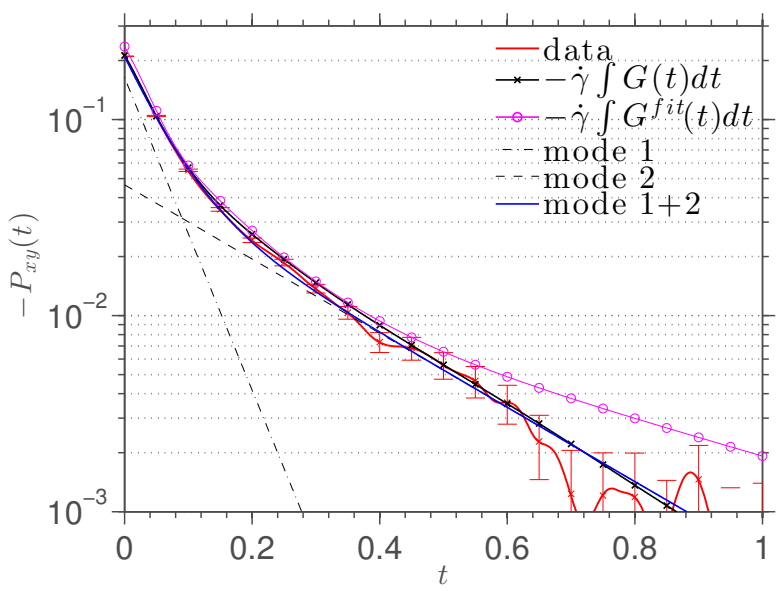

Figure 5.20: In the linear regime ( $\dot{\gamma}=0.1$ shown), the relaxation of shear stress after cessation of steady shear can be accurately predicted from equilibrium statistical mechanics. The sum of two exponential relaxation modes also fit the data well $(\rho=$ $0.84, T=1.0)$. The fitting parameters are $C=0.8041, \tau_{1}=0.0565$ and $\tau_{2}=0.2482$.

by substituting the fit of the shear modulus of a dense fluid (Eq. (5.32) ) into Eq. (5.19)

$$
P_{y x}(t) \approx-\dot{\gamma} G^{\infty}\left(\sqrt{\frac{8 A^{2} \tau_{1}^{2}}{\pi}} \operatorname{erfc}\left(\frac{t}{\sqrt{2 \tau_{1}^{2}}}\right)+\tau_{2} B \mathrm{e}^{-\frac{t}{\tau_{2}}}+\tau_{3}(1-A-B) \mathrm{e}^{-\frac{t}{\tau_{3}}}\right)
$$

The shear stress calculated from this expression, with the fitting parameters reported in Table 5.1, is shown in Figure 5.20. This integrated fit shows good agreement with the shear stress at short times, but deviates at longer times. This may be expected since the fit of the shear modulus (Figure 5.5(b) showed a slower decay than the data in this region, which correspond to an over-prediction of the shear stress. Furthermore, both modes of the double exponential fit (Eq. (5.62), and the combination of the modes are shown in the figure. The fit is shown to be in good agreement with the shear stress data, with fitting parameters $C=0.8041, \tau_{1}=0.0565$ and $\tau_{2}=0.2482$. However, two exponential relaxation functions are not enough to capture all the mechanisms related to structural relaxation of a dense liquid, since we have seen that the stress relaxation modulus shows an initial Gaussian-like shape (Figure 5.4), followed by multiple relaxation modes. Especially at very short times, the slope of the fit deviates from that of the stress relaxation profile, while the profile calculated with Eq. 5.19 is in perfect agreement.

The relaxation time obtained from Eq. (5.28) $(\tau=0.098)$ lies in between the relaxation times of the two fitted exponential modes. This is because the overall relaxation time is a weighted average of the spectrum of relaxation modes present in the fluid. An 
example to illustrate this point can be given by taking the time derivative of Eq. 5.62 and defining a time scale as $\tau_{*}^{-1}=\dot{P}_{y x}(0) / P_{y x}(0)$

$$
\frac{\dot{P}_{y x}(0)}{P_{y x}(0)}=\frac{1}{\tau_{*}}=\left(\frac{C}{\tau_{1}}+\frac{1-C}{\tau_{2}}\right) .
$$

This results in a different time scale $\left(\tau_{*}=0.067\right)$ than defined by Eq. (5.28). We know that if linear viscoelastic theory is valid $\dot{P}_{y x}(0)=-\dot{\gamma} G^{\infty}$ and $P_{y x}(0)=-\dot{\gamma} \eta$, where $\eta$ is close to the Newtonian viscosity. For small enough shear rates, such that the stress response is a linear function of the shear rate, the time scale defined in Eq. (5.64) becomes $\tau_{*}=\eta / G^{\infty}$. This expression is equal to the Maxwell relaxation time that is often used as the characteristic time in the linear viscoelastic Maxwell model [258. This model is based on the assumption that the relaxation function can be accurately described with a single exponential. We have shown that this is not the case for any of the fluid densities studied here.

We now briefly discuss the stress relaxation from a steady shear flow at a shear rate $\dot{\gamma}=1.0$. At high shear rates, the shear stress is not linearly proportional to the strain rate and linear viscoelastic theory is expected to break down. Non-Newtonian phenomena, such as shear thinning, shear dilatancy and normal stress differences, are known to occur for shear rates of this magnitude. Figure 5.21 confirms that the linear viscoelastic theory is not a good approximation at this high shear rate. The shear stress relaxes faster than linear viscoelastic theory predicts. Furthermore, the linear theory does not account for the shear thinning that is responsible for the deviation between the data and the EMD prediction for the shear stress at $t=0$ (the steadystate shear stress before relaxation takes place). The double exponential fit, with fitting parameters $C=0.9452, \tau_{1}=0.0473$ and $\tau_{2}=0.2271$, is still a good approximation of the shape. The second relaxation time $\tau_{2}$ is almost equal at both shear rates, while the first relaxation time $\tau_{1}$ is smaller at the high shear rate, corresponding to a faster stress decay. Furthermore, the first mode is initially more dominant at high shear rate compared to the lower shear rate, i.e. larger value for $C$.

Figure 5.22 shows the relaxation of the normal stresses after cessation of steady shear flow at strain rate $\dot{\gamma}=1.0$. Each of the normal stress components (and thus also the hydrostatic pressure, defined as $p=\frac{1}{3} \operatorname{tr}(\mathbf{P})$ ) decays as the fluid relaxes to equilibrium. This demonstrates the existence of stress dilatancy in the system. Furthermore, the normal stresses are not equal to each other at $t=0$, which shows the presence of normal stress differences (shown in the inset). The relaxation of the normal stress components is different from the relaxation of shear stress. They converge to equilibrium at a lower rate than the shear stress. Furthermore, the normal stresses converge to each other much faster than they relax to equilibrium, as shown in the inset. Heyes et al. 256] studied, for a sheared soft fluid under startup flow, the structural rearrange- 


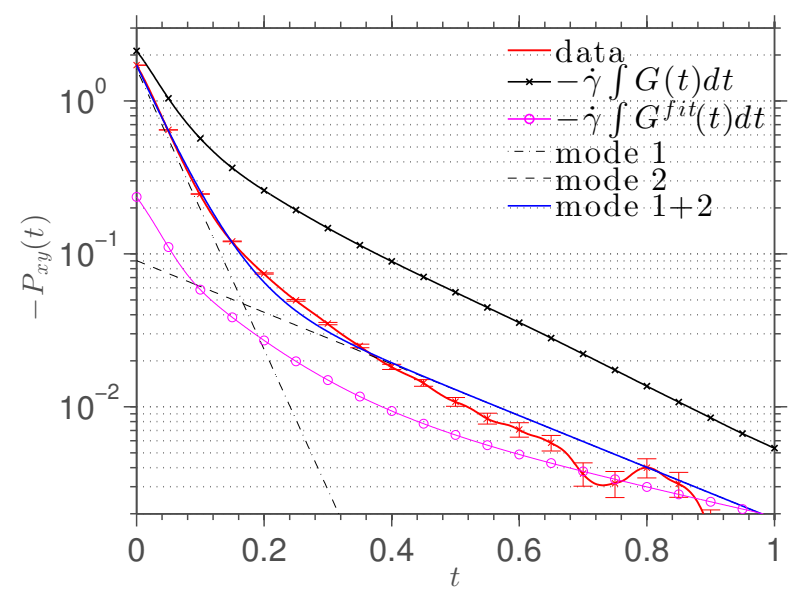

Figure 5.21: Shear stress relaxing from steady shear flow at $\dot{\gamma}=1.0$. The data is fitted with a double exponential curve. The fitting parameters are $C=0.9452, \tau_{1}=0.0473$ and $\tau_{2}=0.2271$.

ment of the fluid. They found that the various contributions to the distortion of the pair distribution function were not equally fast. The $x y$ contribution to the distortion developed much faster than the $x^{2}, y^{2}$ and $z^{2}$ terms, where the latter developed again much slower than the other terms. These observations are supported by our stress profiles for cessation flow, the normal stresses decay much slower than the shear stress and the initial decay rate of $P_{z z}$ is smaller than that of $P_{x x}$ and $P_{y y}$. The relaxation of the normal stress components is fitted with the sum of two exponential modes. The differences between the fits of normal stress components agree well with the relaxation of the normal stress differences. We have observed that these nonlinear transient effects behave very differently in startup flow, compared to non-driven relaxation from a non-equilibrium steady state. The stress response in a startup flow is not shown here, however, for example in Ref. [28], transient normal stress differences are shown for atomic shear flow simulations $(\dot{\gamma}=1.0)$, performed at the same state point as our simulations.

\subsection{Summary and conclusions}

Viscoelasticity and the calculation of material constants were introduced in this chapter, as well as the second-order fluid model that predicts some non-Newtonian fluid phenomena. Existing theories, in conjunction with transient and steady state molecular dynamics simulations of homogeneous simple atomic fluids have been used to gain 


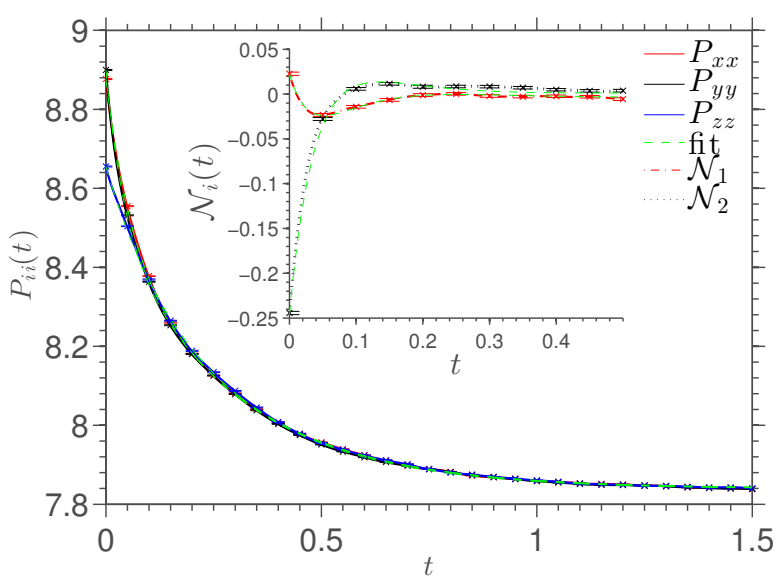

Figure 5.22: Relaxation of the diagonal components of the pressure tensor and the normal stress differences after a sudden cessation of steady shear flow $(\dot{\gamma}=1.0)$. At $t=0, P_{x x}$ and $P_{y y}$ are almost identical, while $P_{z z}$ is significantly lower. The relaxation of the normal stresses are fitted with the sum of two exponentials. The normal stress differences are shown in the inset.

understanding of: (1) the density-dependence of equilibrium material constants, (2) the rheology of these fluids under various types of steady-state flows, and (3) the transient stress and viscosity behavior in startup flow and fluid that relaxes to equilibrium.

Very accurate calculations of the shear stress relaxation modulus of a simple atomic fluid over a wide range of densities have been presented. The zero-shear rate viscosity, zero-shear rate first normal stress coefficient and relaxation time are calculated from the appropriate integrals over the stress autocorrelation function. The accuracy of our EMD simulation data enables us to study the shape of the relaxation function, the density dependence of the material functions, and to make a quantitative comparison between molecular dynamics simulation results and kinetic theory.

Little is known about the functional form of the relaxation modulus. We have shown that the shear modulus of a dilute simple fluid can be accurately fitted with the sum of a Gaussian and an exponential. The width of the Gaussian shows very little variation with density, whereas the decay rate of the exponential term increases with density in the dilute regime. This leads to a decrease of the relaxation times for densities up to $\rho=0.68$. At higher densities, the behavior becomes more complicated, due to the development of a slowly-decaying tail in the shear modulus. This tail leads to an increase of the relaxation time with density.

The shear stress relaxation functions for fluids at various densities have been used to calculate zero-shear rate viscosities, infinite-frequency shear moduli and zero-shear 
rate first normal stress coefficients. We have shown that the zero-shear rate viscosity and the infinite-frequency shear modulus increase monotonically with density. Both density dependences show good agreement with data from the literature. Furthermore, the viscosity in the dilute limit agrees well with the predicted value from kinetic theory.

The zero-shear rate first normal stress coefficient shows a qualitatively similar density dependence to the relaxation times. The zero-shear rate first normal stress coefficient decreases with increasing density at low densities and strongly increases with density in the dense regime, while a minimum normal stress coefficient is found around $\rho=0.52$. The decaying trend in the dilute regime is in good agreement with the prediction from kinetic theory. This agreement is a confirmation of the validity of our MD simulations as well as the theory presented by Coleman and Markovitz [216]. In the dense region, the viscoelastic memory effect causes an increase in the normal stress coefficient towards the solid-liquid transition density.

Many studies in the literature have focussed on either shear flow or elongational flows. Only very few works have been devoted to a combination of shear flow and planar elongational flow of simple atomic fluids. This type of flow, referred to as planar mixed flow, makes it possible to study fluid rheology for a wide variety of planar flows. Non-Newtonian models that are applicable for arbitrary flows have seen little success to date. Tensor expansions in terms of the strain rate tensor exist (e.g. the second-order fluid model), but these models are based on equilibrium quantities and do not account for shear thinning. In this chapter, the limitations of the second-order fluid model have been shown and an alternative model has been presented.

We have presented a framework to predict the pressure tensor of a fluid under a steady homogeneous planar flow. The framework describes the full pressure tensor in terms of four variables, which is the minimum number of variables required. This framework involves a quantitative description of various non-Newtonian phenomena, i.e., strain-rate thinning viscosity, pressure dilatancy, viscoelastic lagging and out-ofplane axial pressure tensor anisotropy.

Steady-state non-equilibrium molecular dynamics simulations of a WCA fluid near the LJ triple point have been performed. Simulations for shear flow, planar elongational flow and combined shear and elongation show the same deviations from Newtonian behavior: All of these results, when expressed in terms of functions of the driving field strength $s$, the vorticity $\omega$ and the equilibrium fluid properties, collapse onto master curves showing that the pressure tensor can be predicted for each planar flow without the need of performing NEMD simulations under the given velocity field. Prior knowledge is required about the equilibrium properties and the non-equilibrium scaling of the fluid. These are dependent on its state point.

All NEMD simulation data presented in this chapter corresponded to a single 
density and temperature. The functional form of each of the non-Newtonian quantities have been validated at five different state points. The results of these simulations are listed in Appendix $B$. It was found that each of the fitting parameters depend strongly on the state point of the fluid. An extensive study at various state points could provide more insight in the dependence of non-Newtonian behavior on density and temperature. This study is currently being undertaken.

We have found that the principal pressure tensor for a simple atomic fluid is solely dependent on the scalar magnitude of the flow field, at the chosen state point of the fluid. Furthermore, the Cartesian pressure tensor is also dependent on the shear rate, the vorticity and the principal orientation of the strain rate tensor. However, the orientation of the pressure tensor is defined relative to the orientation of the strain rate tensor, not explicitly dependent on the Cartesian coordinate system.

The model is calibrated with results from various simulations over a wide range of deformation rates. Furthermore, the model was used to predict (extrapolate) the pressure tensor for simulations much further away from equilibrium and very close to equilibrium. The predictions were very accurate for all model variables up to large deformation rates.

A possible next step towards a 'complete' description of the non-Newtonian pressure tensor should be an extension of the model to allow for transient or time-dependent flows. This would require an extensive study of relaxation to non-equilibrium steady states and to equilibrium. Preliminary studies show a similar trend and relaxation times in the linear response regime, but distinctive differences when the response is a non-linear function of the deformation rate (i.e., in the shear thinning regime). The range of deformation rates considered in this study is far into the non-linear regime, where transient behavior is extremely complicated. In order to attempt an extension towards a time-dependent description of the pressure tensor, a study with lower deformation rates is advised.

The transient shear stress and shear viscosity after imposing a sudden constant deformation on a simple atomic fluid have been measured from NEMD simulations for several shear rates in the range $\dot{\gamma} \in[0.1,1.0]$. The shear response of a WCA fluid at this state point $(\rho=0.8442, T=0.722)$ under these shear rates is a non-linear function of the shear rate (i.e., in the shear thinning regime), as confirmed by the steady state viscosity calculations. The non-linear part of the transient response is given by the difference between the transient viscosity profiles and the Green-Kubo integral for shear viscosity. The Green-Kubo relation for shear viscosity does not predict nonNewtonian effects, such as shear thinning. At high shear rates, the shear stress and viscosity show an overshoot, after which it converges to its steady-state value.

Linear viscoelastic theory has been applied to predict the linear relaxation of the 
stress from a steady-state shear flow. The prediction made from equilibrium molecular dynamics results have been compared with molecular dynamics simulations of a fluid relaxing from a non-equilibrium steady state. Good agreement was found for a fluid relaxing from a steady flow at shear rate $\dot{\gamma}=0.1$, whereas for a shear rate $\dot{\gamma}=1.0$ the shear stress relaxes faster than the linear theory predicts. Furthermore, the shear thinning that occurs at a large shear rate is not accounted for in the linear viscoelastic theory. At both shear rates, the shear stress can be fitted reasonably well with the sum of two exponential modes. However, the shape of the relaxation modulus suggests that the double exponential fit does not capture the stress relaxation correctly at short times. A more accurate fit was achieved by accounting for the Gaussian shape at short times and the tail at longer times. 


\section{6}

\section{Transient-time correlation functions applied to atomic and molecular fluids}

Thermodynami】quantities of a non-equilibrium steady state flow are often calculated by performing a time-average of the simulation data. The statistical noise associated with instantaneous thermal fluctuations decreases with an increasing amount of data used for the calculation of the average. This approach is efficient to calculate nonequilibrium quantities (such as shear stress or normal stress differences) if the signalto-noise ratio is high (i.e., the thermal fluctuations in an observable are small relative to its average value). A high signal-to-noise ratio is achieved by applying a large deformation rate to the fluid. On the other hand, if the deformation rate is very small, very long time-averages are needed in order to obtain good statistics, making this method not practically feasible. Furthermore, enhancing the statistics by timeaveraging data is limited to steady-state flow problems, while for many flow processes in industry it is also important to study the transient behavior of fluids, for example for pumps and valves. Transient stresses of molecular fluids have been studied widely with molecular dynamics simulations over the last decades [258, 270].

Rudisill and Cummings 271] have used ensemble averages of non-equilibrium molecular dynamics (NEMD) simulations to study the influence of the internal degrees of freedom of dimers on the transient and steady-state shear rheology of alkanes. They compared rigid dimers to freely jointed finitely extensibly (FENE) chains and

This chapter is based on:

R. Hartkamp, S. Bernardi, and B. D. Todd (2012), J. Chem. Phys. 136, 064105,

R. Hartkamp, S. K. Kannam, S. Bernardi, P. J. Daivis, D. J. Searles, and B. D. Todd (2013), in preparation 
a Lennard-Jones fluid. The molecular models showed qualitatively and quantitatively comparable results, while the atomic fluid showed a stronger non-Newtonian behavior, such as shear-thinning. At low strain rates, the calculated viscosity of the rigid molecules was much higher than that of the FENE dumbbells. In a later study [272], the same authors extended their research to include the effect of excluded volume, hydrodynamic interactions and the influence of the chain length on the shear rheology of the fluid. It was found that longer chain molecules start to behave non-Newtonian at lower strain rates than shorter molecules. Furthermore, the internal degrees of freedom of FENE chains did not appear to have a large influence on the shear rheology of a polymer melt.

In the aforementioned work, data has been accumulated by ensemble averaging over several transient NEMD simulations, where large strain-rates are required for a high signal-to-noise ratio. Molecular dynamics simulations are typically driven by forces that are several orders of magnitude larger than those accessible in typical experiments, and thus not very suitable for mimicking experiments on a one-to-one basis. Techniques such as temperature-time superposition 215] can be used to compare NEMD results to experiments, confirming the high accuracy of such simulations. Bair et al. [273] have applied this method successfully to compare NEMD shear flow simulations of low-molecular-weight fluids to experimental data.

In an attempt to reduce the noise in direct NEMD averages, Ciccotti and coworkers 274 276] devised a method in which the thermal fluctuations are subtracted from the non-equilibrium trajectories. This is done by performing an equilibrium simulation for each non-equilibrium trajectory, where both trajectories start from the same point in phase space. Since both trajectories start from the same state, the fluctuations in the trajectories have a perfect correlation at first. This correlation vanishes rapidly due to the Lyapunov instability that drives the systems away from each other at an exponential rate. As the correlation between the equilibrium and non-equilibrium trajectories vanishes over time, so does the advantage of the subtraction method. Hence, the subtraction method does not lead to a more accurate calculation of steady state values than those that are calculated with conventional direct NEMD averaging [277.

The transient-time correlation function (TTCF) [28, 176, 189] method offers a more efficient way to study the rheology of fluids close to equilibrium and make a direct comparison to experimental data. This method is based on the time-correlation between the initial rate of energy dissipation and the transient response of an observable after a driving force is imposed on the fluid.

The transient response of shear flow [27, 44, 277, 279] and various types of shear-free flows [143, 280, have been studied in the past for simple atomic systems. In atomic 
fluids, non-Newtonian behavior typically occurs only under deformation rates that are much larger than those present in most industrial applications. However, many applications contain complex fluids, which show a deviation from Newtonian behavior at very small external fields. Therefore, it is of paramount importance for a one-to-one comparison of simulations to industrial and biological processes to have a method at hand that can predict the fluid response as a non-linear function of the driving force. In recent years, TTCF has been applied, for the first time, to a molecular fluid in a homogeneous shear flow. Pan and McCabe [281, and later Mazyar et al. 282, have applied TTCF to calculate the shear viscosity of $n$-decane.

This chapter is organized as follows: In Section6.1, we give a derivation of the transient-time correlation function and discuss the effect of instantaneous fluctuations on the accuracy of the calculation. TTCF is used to calculate the stresses and viscosity in a simple atomic fluid under planar mixed flow in Section6.2. In Section6.3, normal stress differences in shear flow are calculated using TTCF. In Section6.4. TTCF is applied to short chain molecules under various flow types. The chapters ends with some concluding comments in Section 6.5

\subsection{Transient-time correlation function}

The transient-time correlation function was briefly introduced in Section 4.5. Here we briefly follow the derivation of the TTCF formalism as described by Evans and Morriss in a number of papers [27, 144, 277, 278, 283]. We then use TTCF to calculate components of the pressure tensor and viscosity for atomic and molecular liquids under various types of flow.

The TTCF formalism predicts the ensemble average of a field-dependent phase variable $B(t)$ (denoted as a scalar here, but can be a tensor as well), which can be a component of the pressure tensor for example. The value of $B$ is expressed as a function of time $t$, which is only an implicit dependence 1 via the $N$-particle phase space vector $\boldsymbol{\Gamma}=\left(\mathbf{r}_{1}, \ldots, \mathbf{r}_{N}, \mathbf{p}_{1}, \ldots, \mathbf{p}_{N}\right)$, such that we write $B(t) \equiv B(\boldsymbol{\Gamma}(t))$. The same argument applies to the phase space distribution function $f(t) \equiv f(\boldsymbol{\Gamma}(t))$. The evolution of $B(t)$ subject to a homogeneous driving force (activated at $t=0$ ) can be given by the time-derivative of the Heisenberg representation of a phase-space average

$$
\frac{d\langle B(t)\rangle}{d t}=\frac{d}{d t} \int f(0) B(t) d \boldsymbol{\Gamma}=\int f(0) \dot{\boldsymbol{\Gamma}} \cdot \frac{\partial B(t)}{\partial \boldsymbol{\Gamma}} d \boldsymbol{\Gamma} .
$$

The phase variable is propagated via the phase variable propagator $\exp (\mathrm{iL} t)$, which relates the current value of a phase variable to the initial value via $B(t)=\exp (\mathrm{iL} t) B(0)$, where iL is the $p$-Liouvillean. This implies that the current value of the phase variable

\footnotetext{
${ }^{1}$ We consider only cases in which the driving force is constant in time.
} 
depends only on the initial value and on the equations of motion and not on the current phase-space vector.

Integrating Eq. (6.1) by parts gives

$$
\frac{d\langle B(t)\rangle}{d t}=[f(0) \dot{\boldsymbol{\Gamma}} B(t)]_{S}-\int B(t) \frac{\partial}{\partial \boldsymbol{\Gamma}} \cdot(f(0) \dot{\boldsymbol{\Gamma}}) d \boldsymbol{\Gamma},
$$

where the surface term is zero [278. Integrating Eq. 6.2 with respect to time gives

$$
\langle B(t)\rangle=\langle B(0)\rangle-\int_{0}^{t} \int B(s) \frac{\partial}{\partial \boldsymbol{\Gamma}} \cdot(f(0) \dot{\boldsymbol{\Gamma}}) d s d \boldsymbol{\Gamma} .
$$

It was shown in Ref. [280] that for the adiabatic case Eq. 6.3 can be rewritten using

$$
\frac{\partial}{\partial \boldsymbol{\Gamma}} \cdot(f(0) \dot{\boldsymbol{\Gamma}})=i \mathcal{L} f(0)=\beta \mathbf{J}(0): \mathbf{F}_{e} f(0)=\beta V(\mathbf{P}(0): \nabla \mathbf{u}) f(0)=-\beta \dot{\mathcal{H}}^{a d}(0) f(0),
$$

where $i \mathcal{L}$ is the $f$-Liouvillean, $\beta=1 /\left(\mathrm{k}_{\mathrm{B}} T\right), \mathbf{J}(0)$ the initial dissipative flux, $\mathbf{F}_{e}$ the external driving force and $\dot{\mathcal{H}}^{a d}(0)=-V(\mathbf{P}(0): \nabla \mathbf{u})$ is the rate of energy dissipation of the adiabatic system when the field is turned on, before the extraction of viscous heat becomes required to maintain a constant temperature. The rate of energy dissipation can be calculated from the SLLOD equations of motion (Eqs. (3.6) and (3.7)) presented in Chapter 3. Substituting Eq. 6.4) into Eq. 6.3 results in the TTCF formulation for a general homogeneous flow

$$
\begin{aligned}
\langle B(t)\rangle & =\langle B(0)\rangle-\beta V \int_{0}^{t}\langle B(s)(\mathbf{P}(0): \nabla \mathbf{u})\rangle d s \\
& =\langle B(0)\rangle+\int_{0}^{t}\langle B(s) \Omega(0)\rangle d s,
\end{aligned}
$$

where $\Omega(0) \equiv-\beta V \mathbf{P}(0): \nabla \mathbf{u}$.

If the system exhibits mixing [277], then the instantaneous phase variable $B(t)$ becomes eventually $(t \rightarrow \infty)$ uncorrelated to the initial rate of energy dissipation $\mathbf{P}(0): \nabla \mathbf{u}$, leading to $\langle B(t) \Omega(0)\rangle \sim\langle B(t)\rangle\langle\Omega(0)\rangle$. The ensemble average of the initial rate of energy dissipation $\langle\Omega(0)\rangle$ approaches zero in the statistical limit for infinitely many trajectories or atoms. If this is the case, then the correlation function decays to zero and the TTCF integral converges. However, due to numerical inaccuracy $\langle B(t)\rangle\langle\Omega(0)\rangle \neq 0$ and thus the integral never converges to a constant value. In order to account for this numerical inaccuracy, we can subtract the error as follows

$$
\begin{aligned}
\langle B(s)(\Omega(0)-\langle\Omega(0)\rangle)\rangle & =\langle B(s) \Omega(0)\rangle-\langle B(s)\rangle\langle\Omega(0)\rangle \\
& =\langle(B(s)-\langle B(s)\rangle) \Omega(0)\rangle \\
& =\langle\Delta B(s) \Omega(0)\rangle
\end{aligned}
$$


where $\Delta B(s)=B(s)-\langle B(s)\rangle$ denotes the instantaneous fluctuations in $B(s)$. Note that this expression goes to zero when $B(t)$ and $\Omega(0)$ become uncorrelated.

In the special case that the initial average rate of energy dissipation $\langle\Omega(0)\rangle$ is exactly zero, the correlation can be rewritten to $\langle B(s) \Omega(0)\rangle=\langle\Delta B(s) \Omega(0)\rangle$. This condition is only exactly satisfied in the case of a suitable phase-space mapping, which will be discussed in Section 6.2.1. Substituting the corrected correlation function (Eq. (6.9)) into Eq. (6.5) gives

$$
\langle B(t)\rangle=\langle B(0)\rangle-\beta V \int_{0}^{t}\langle\Delta B(s) \Omega(0)\rangle d s
$$

which is, in general, not identical to Eq. 6.6.

Evans and Morriss 28, 252 have studied the possibility of combining the TTCF method with the subtraction method of Ciccotti and coworkers [274 276]. This resulted in the differential TTCF method, in which an equilibrium trajectory is subtracted from $\Delta B$. This is essentially the same as subtracting the Green-Kubo linear response from Eq. 6.10), leaving only the nonlinear part of the response of $B(t)$. The authors showed that the field-free contribution of the differential TTCF method is exactly zero such that the result is identical to that of the conventional TTCF expression shown in Eq. 6.10.

\subsection{Planar Mixed Flow}

In the past, TTCF has been applied to atomic shear [277, 278] and elongational [280] flows. In this section, TTCF is applied to a planar mixed flow with the purpose of decreasing the gap between NEMD simulations and industrial flow problems. Many industrial processes are performed at much smaller deformation rates than those used in typical NEMD simulations and with velocity profiles that are often caused by a combination of multiple types of deformation on the fluid.

The equations of motion of an atomic fluid under homogeneous planar mixed flow (PMF) were shown in Chapter 3 as

$$
\begin{aligned}
\dot{\mathbf{r}}_{i} & =\frac{\mathbf{p}_{i}}{m_{i}}+\dot{\epsilon}\left(x_{i} \mathbf{e}_{x}-y_{i} \mathbf{e}_{y}\right)+\dot{\gamma} y_{i} \mathbf{e}_{x}, \\
\dot{\mathbf{p}}_{i} & =\mathbf{F}_{i}-\dot{\epsilon}\left(p_{x i} \mathbf{e}_{x}-p_{y i} \mathbf{e}_{y}\right)-\dot{\gamma} p_{y i} \mathbf{e}_{x}-\zeta \mathbf{p}_{i},
\end{aligned}
$$

where $\mathbf{e}_{\alpha}$ is a unit vector oriented in the $\alpha$-direction, $\alpha=x, y, z, \alpha_{i}$ is the position of atom $i$ and $p_{\alpha i}$ is its peculiar momentum in the $\alpha$-direction and $\zeta$ is the thermostat multiplier. Each atom feels the external field at the same time. The explicit external field in combination with the homogeneous character makes the SLLOD equations of motion amenable to response theory. 
Combining the TTCF formulation (Eq. 6.10) with the rate of energy dissipation (Eq. (6.4) and the velocity gradient for PMF (Eq. (3.37)) results in the nonlinear response of a phase variable $B$ that evolves under PMF [254]

$$
\langle B(t)\rangle=\langle B(0)\rangle-\beta V\left(\dot{\epsilon} \int_{0}^{t}\left\langle\Delta B(s)\left(P_{x x}(0)-P_{y y}(0)\right)\right\rangle d s+\dot{\gamma} \int_{0}^{t}\left\langle\Delta B(s) P_{x y}(0)\right\rangle d s\right) .
$$

The nonlinear response of observable $B(t)$ evolving under PMF will, in general, not be the same as the superposition of the nonlinear response of separate planar shear and planar elongation simulations. The reason for this is that the nonlinear response contains the product of the instantaneous phase variable $B(t)$ (evolving under PMF), as well as the initial dissipation $\Omega$ for PMF. Since both quantities are field-dependent, the product does not simply relate to the field in a linear fashion. For the linear response, the superposition would be valid, since the dissipation for PMF is simply a linear combination of that of planar Couette flow (PCF) and planar elongational flow (PEF) and the non-equilibrium (field-dependent) values of the phase variable are not included in the linear response formulation.

\subsubsection{Simulation details}

We simulate an atomic fluid whose interactions are mediated via a Weeks-ChandlerAndersen (WCA) 77] potential and the equations of motion are integrated with a fourth-order Runge-Kutta scheme with a time-step of $\Delta t=0.001$. This algorithm is self-starting, which is an important property for the study of transient behavior [277]. All physical quantities presented are reduced using the particle mass $m$, interaction length scale $\sigma$ and the potential energy well-depth $\epsilon$. These scales are set to unity. The phase point of the fluid is set close to the Lennard-Jones triple point, $\rho=0.8442$ and $T=0.772$, where the properties of simple fluids (like Argon) are well-known. ${ }^{2}$ To maintain a constant temperature, the generated heat needs to be removed from the system. This is done via the Gaussian isokinetic thermostat [114.

The initial cell vectors that span the simulations cell in the plane of deformation have lengths 14.43 and 8.43 and a relative angle of 90 degrees (for our simulation with equal rates of shear and elongational). A third lattice vector, oriented in the $z$-direction, has a length of 8.69. As time advances, the simulation cell is deformed in in the $x-y$ plane. Since the cell size has to be at least twice the cut-off distance of the potential $\left(2^{1 / 6}\right.$ for the WCA potential) in each direction (in order for atoms not to interact with their periodic image), the maximum simulation time would be limited by the cell size in the contraction direction, regardless of the initial cell-size. Hunt et al. [32 introduced a set of boundary conditions (see Section 3.4 that remap the positions

\footnotetext{
${ }^{2}$ This is a widely studied state point also for WCA fluids.
} 
of the atoms to the initial simulation cell after a certain time without disturbing the flow. These boundary conditions avoid the time limit due to the deformation of the cell and allow for indefinitely long simulations.

Before the external field is activated and the transient response can be calculated, the simulation is relaxed to equilibrium. This results in an initial state for a nonequilibrium simulation $\boldsymbol{\Gamma}_{1}=\left(\mathbf{x}, \mathbf{y}, \mathbf{z}, \mathbf{p}_{x}, \mathbf{p}_{y}, \mathbf{p}_{z}\right)$, where each component is a vector with length $N$. If a system exhibits mixing, we can modify the initial state in specific ways, such that another initial state is created with equal probability and internal energy. This procedure is called phase-space mapping [28, 278]. The phase-space mapping provides additional initial configurations with the purpose of creating more field-dependent trajectories from an equilibrium phase point. These additional trajectories enhance the statistical accuracy.

If a mapping can be created that exactly satisfies $\sum_{M}\left(\mathbf{P}\left(\boldsymbol{\Gamma}_{M}(0)\right): \nabla \mathbf{u}\right)=0$ (where the summation is over the non-equilibrium starting states), the phase-space mapping eliminates the numerical uncertainty that leads to a non-zero initial rate of energy dissipation [278. Hence, $\langle\Omega(0)\rangle=0$ would be exactly satisfied, making the subtraction shown in Eq. 6.7) redundant. If the pressure tensor is symmetric at time $t=0$ (before the field is activated), the phase vector $\boldsymbol{\Gamma}_{M}=M^{P M F}\left(\boldsymbol{\Gamma}_{1}\right)=\left(-\mathbf{y}, \mathbf{x}, \mathbf{z},-\mathbf{p}_{y}, \mathbf{p}_{x}, \mathbf{p}_{z}\right)$ satisfies the condition given above for PMF. The off-diagonal pressure term $P_{x y}$ is mapped to $-P_{y x}$, which cancel out against each other in the sum over the nonequilibrium starting states in the case that $P_{x y}(0)=P_{y x}(0)$. The pressure tensor for an atomic fluid is inherently symmetric. This is however not always the case for molecular fluids, in which case an additional phase-space mapping is required. In addition to satisfying this condition, a suitable phase-space mapping should not conflict with the boundary conditions. Since boundary conditions for a generic flow type do not exist that will guarantee indefinite simulation times, the phase-space mapping needs to be addressed on a case-to-case basis. Indefinite simulations of PMF, in general, require non-orthogonal cells, which makes it very difficult to remap positions without changing the relative distances between atoms and thus disturbing the initial conditions of the flow. Furthermore, the flow field does, in general, not align with the lattice vectors. This can be the case even if a cell is orthogonal, for example in PEF with the Kraynik-Reinelt periodic boundary conditions [153].

Figure 6.1 illustrates the problem that is associated with a phase-space mapping of the coordinates of particles when the cell is not aligned with the Cartesian axes. The figure shows a $y$-reflection mapping [28] in a rectangular cell. The positions of two points before mapping are denoted with $\left(1, t_{0}\right)$ and $\left(2, t_{0}\right)$. The $y$-reflection mapping reflects the $y$-coordinates of each point in the cell with respect to the $x$-axis (i.e., all the positions of the mapped points lie within the red area), and the new positions 


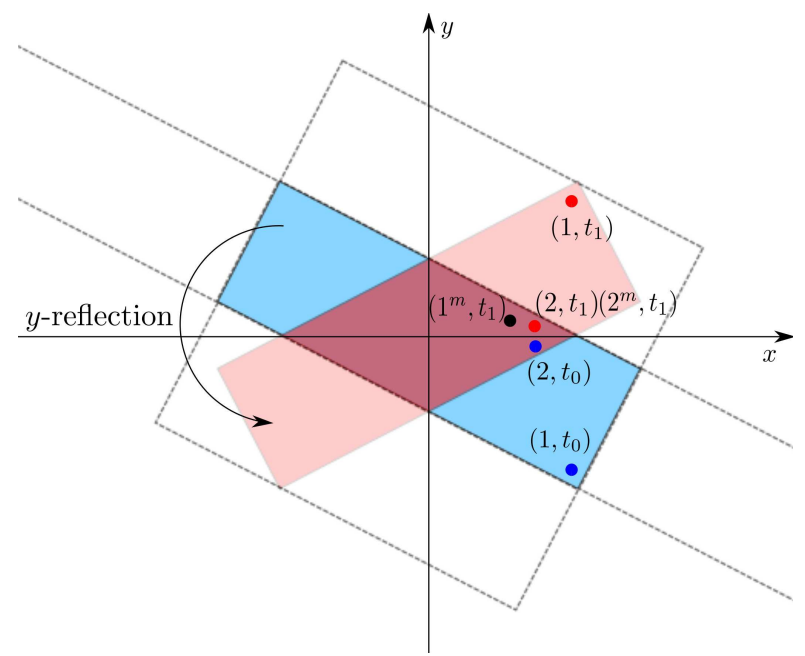

Figure 6.1: $y$-Reflection mapping in a rectangular cell oriented by some non-zero angle with respect to the flow fields. The position of two points before mapping are indicated by $t_{0}$. After the $y$-reflection mapping $\left(t_{1}\right)$ and projection onto the initial cell, the relative distance between the points has changed.

of the two points are marked by $\left(1, t_{1}\right)$ and $\left(2, t_{1}\right)$. One of the points is still located in the blue cell, whereas the other point is located in a periodic image of the cell. Mapping the periodic images onto the (blue) cell results in the final positions $\left(1^{m}, t_{1}\right)$ and $\left(2^{m}, t_{1}\right)$. Relative to the initial points $\left(1, t_{1}\right)$ and $\left(2, t_{1}\right)$, the distance between the two points has changed. Hence, the phase-space mapping in this example interferes with the dynamics of the fluid.

Finding a mapping in which the relative distances between atoms remain unchanged is still an open problem for a simulation cell that is not square or aligned with the field directions. However, the correction shown in Eq. (6.7), eliminates the need for this specific phase-space mapping. Changing the sign of all of the momenta does not create a phase vector that satisfies the first condition stated above. It does, however, create a distinct phase-space trajectory. Therefore, the time-reversal mapping $\boldsymbol{\Gamma}_{2}=M^{T}\left(\boldsymbol{\Gamma}_{1}\right)=\left(\mathbf{x}, \mathbf{y}, \mathbf{z},-\mathbf{p}_{x},-\mathbf{p}_{y},-\mathbf{p}_{z}\right)$ is applied to each initial state for the nonequilibrium simulations performed in this study.

In order to obtain good statistics, many non-equilibrium simulations are needed. This is done by running one equilibrium simulation for a long time and branching off many non-equilibrium trajectories, as is shown in Figure6.2. The equilibrium mother trajectory, indicated with 'EQ', provides a starting state $\boldsymbol{\Gamma}_{1}$ for a non-equilibrium daughter trajectory 'NEQ1', while $\boldsymbol{\Gamma}_{1}$ is stored. Another state is created by apply- 


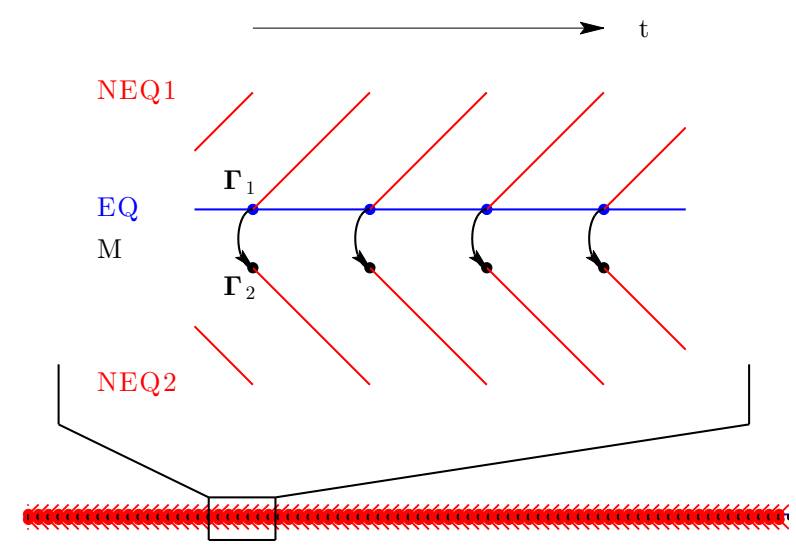

Figure 6.2: A schematic of the equilibrium and non-equilibrium trajectories used for the TTCF calculation. The equilibrium mother trajectory is indicated with 'EQ', the phase space mapping with ' $\mathrm{M}$ ' and the non-equilibrium trajectories created from both starting states are 'NEQ1' and 'NEQ2'.

ing the previously discussed phase-space mapping 'M' to $\boldsymbol{\Gamma}_{1}$. The mapped state $\boldsymbol{\Gamma}_{2}$ serves as the starting state for another non-equilibrium daughter trajectory 'NEQ2'. Both non-equilibrium trajectories contribute to the accumulation of statistics for the transient-time correlation function. It is often also possible to have more than two non-equilibrium trajectories, if sufficient suitable phase space mappings exist that are compatible with the periodic boundary conditions. After the simulations of the nonequilibrium trajectories are done, the equilibrium mother trajectory is continued from the stored state $\boldsymbol{\Gamma}_{1}$ and the cycle is repeated as often as needed to accumulate good statistics. The time between each new set of branches is chosen to be larger than the relaxation time of the pressure auto-correlation function in order to make sure that the different sets of branches are uncorrelated to each other.

\subsubsection{Atomic mixed flow results}

We look at the transient response of the shear stress $B(t)=P_{x y}(t)$ and the normal stresses $B(t)=P_{x x}(t), P_{y y}(t)$ in the plane of deformation. We have chosen to study these stress components since they contribute to the generalized viscosity for PMF.

The nonlinear response is compared to a direct average of the non-equilibrium molecular dynamics simulations, where the instantaneous pressure tensor is calculated 
with the virial stress formulation

$$
\mathbf{P}=\frac{1}{V} \sum_{i=1}^{N}\left(\frac{\mathbf{p}_{i} \mathbf{p}_{i}}{m_{i}}+\frac{1}{2} \sum_{j \neq i} \mathbf{r}_{i j} \mathbf{F}_{i j}\right),
$$

where $\mathbf{p}_{i} \mathbf{p}_{i}$ denotes the dyadic product between the peculiar momentum vectors, $\mathbf{F}_{i j}$ is the interaction force between atoms $i$ and $j$, and $\mathbf{r}_{i j}=\mathbf{r}_{i}-\mathbf{r}_{j}$.

We present simulation results for field strengths $(\dot{\gamma}, \dot{\epsilon}) \in\left\{5 \times 10^{-4}, 0.001,0.005,0.05\right\}$. The shear rate $\dot{\gamma}$ and the elongational rate $\dot{\epsilon}$ are chosen to be equal in our simulations. There is no reason why they should or should not be equal and these values are arbitrarily chosen. Simulations were also run where the shear rate and the elongational rate were not equal (not explicitly shown in this section, but shown for molecular fluids in Section 6.4). These simulations have a different ratio between the shear stress and the normal stress fields, but they show a similar transient behavior. Furthermore, simulations have been run for shear flow and planar elongational flow in order to verify our simulation results with earlier studies [277, 280]. Good agreement was found for both types of flow.

Figures 6.3 and 6.4 show the stress response under planar mixed flow with $\dot{\epsilon}_{x x}=$ $0.05, \dot{\epsilon}_{y y}=-0.05$ and $\dot{\gamma}=0.05$. The direct averages of the instantaneous stresses and the TTCF response are shown. The data is averaged over $10 \times 2 \times 7500$ non-equilibrium trajectories, where the first number indicates the number of distinct simulations, the second is the number of simultaneous non-equilibrium trajectories branched off from an equilibrium state (i.e., the original equilibrium starting state and the mapped starting state) and the last number indicates how many pairs of non-equilibrium trajectories are branched off per simulation. Each trajectory is a simulation containing $N=896$ atoms. The standard error is given by the error bars. The standard error is calculated from the 10 distinct simulations. For this external field, both methods produce smooth, converging profiles which are in good agreement with each other.

For smaller deformation rates, the efficiency of TTCF is expected to become higher than that of direct NEMD averages. Figures 6.5 and 6.6 show the transient stress subject to PMF with $\dot{\epsilon}_{x x}=0.005, \dot{\epsilon}_{y y}=-0.005$ and $\dot{\gamma}=0.005$. The number of nonequilibrium trajectories and the number of atoms are identical to the data shown in Figures 6.3 and 6.4 . The direct NEMD averages fluctuate strongly around an underlying trend, while the TTCF response is again smooth and converges to a steady-state.

One would expect the initial shear stress to be zero and the initial normal stresses equal to the isotropic pressure. In practice, however, the ensemble average is subject to small deviations due to instantaneous fluctuations, as seen in Figures 6.5 and 6.6. but converges in the statistical limit of infinitely many atoms or trajectories. As the uncertainty in the ensemble average of the starting states $\langle B(0)\rangle$ is independent of the 


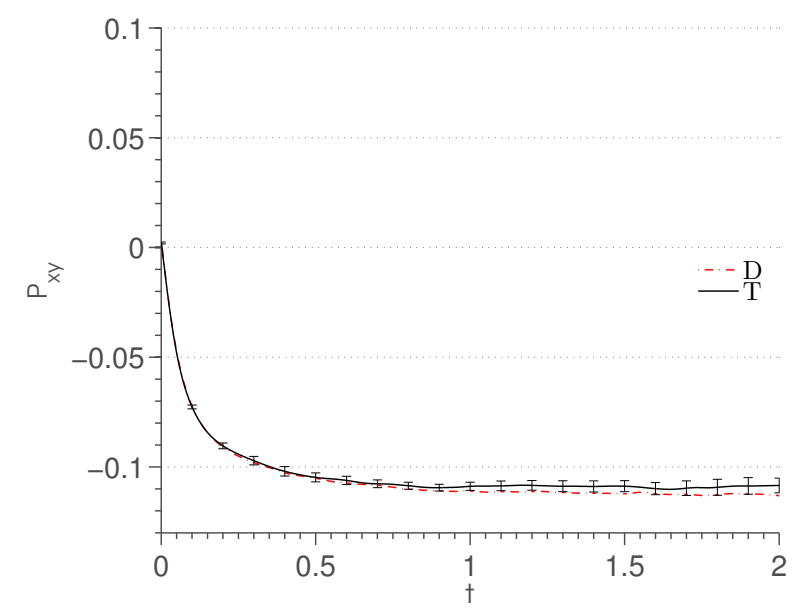

Figure 6.3: $B=P_{x y}$ for mixed flow with $\dot{\gamma}=0.05$ and $\dot{\epsilon}=0.05$. The label ' $\mathrm{T}$ ' indicates the TTCF result and ' $\mathrm{D}$ ' the direct average over the same non-equilibrium trajectories.

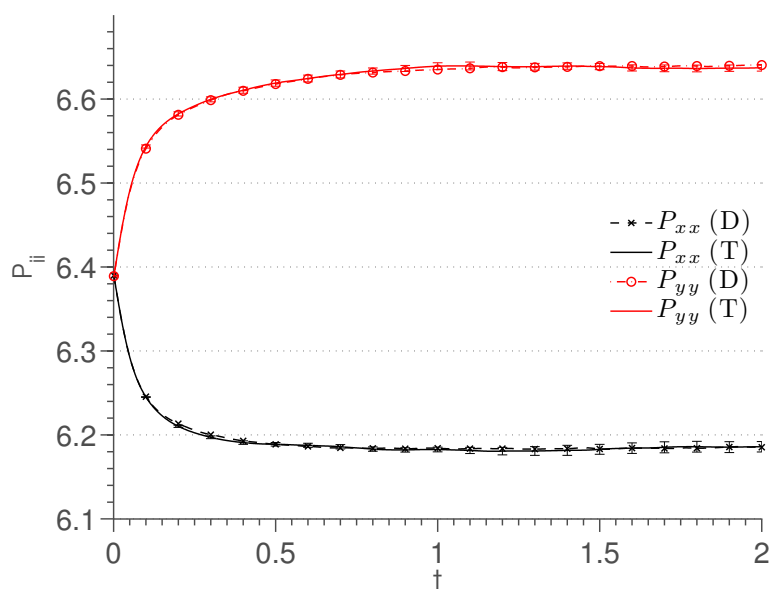

Figure 6.4: Normal stress response under planar mixed flow with $\dot{\epsilon}_{x x}=0.05, \dot{\epsilon}_{y y}=$ -0.05 and $\dot{\gamma}=0.05$. The label ' $\mathrm{T}$ ' indicates the TTCF result and ' $\mathrm{D}$ ' the direct average over the same non-equilibrium trajectories. 


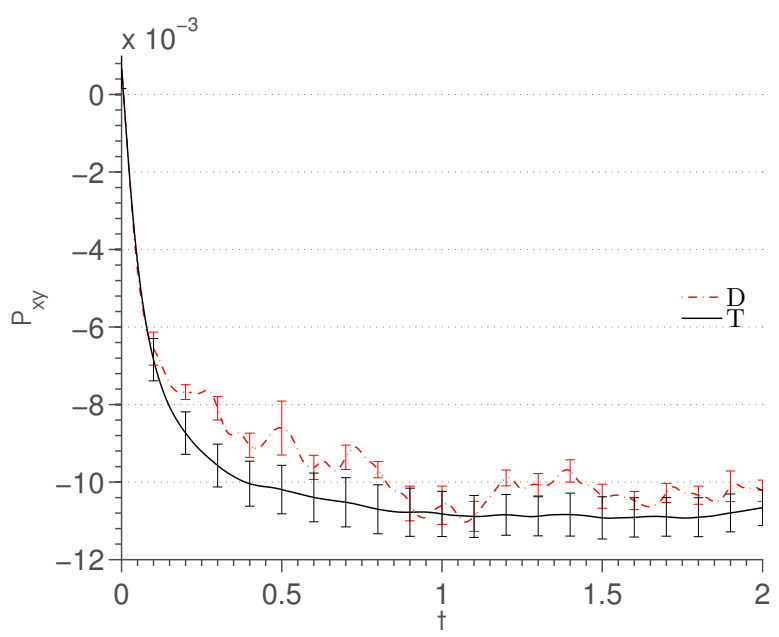

Figure 6.5: $B=P_{x y}$ for mixed flow with $\dot{\gamma}=0.005$ and $\dot{\epsilon}=0.005$. The label ' $\mathrm{T}$ ' indicates the TTCF result and ' $\mathrm{D}$ ' the direct average over the same non-equilibrium trajectories.

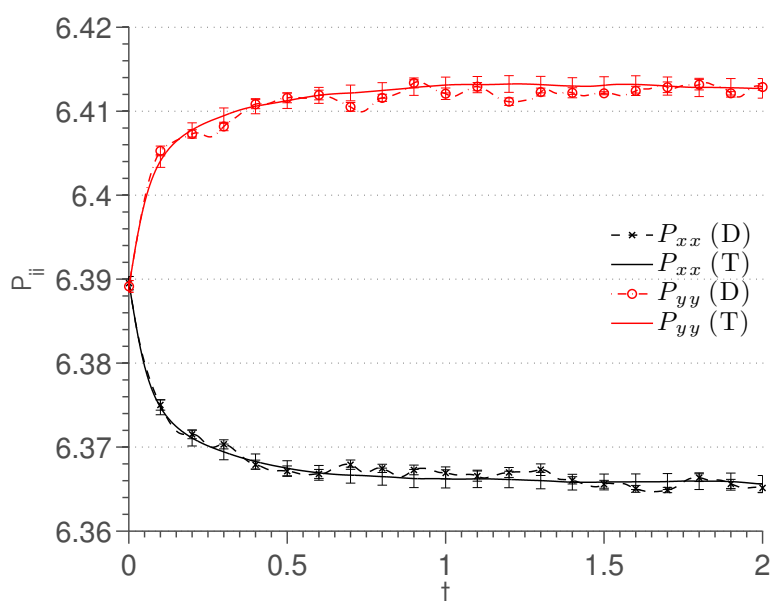

Figure 6.6: Normal stress response under planar mixed flow with $\dot{\epsilon}_{x x}=0.005, \dot{\epsilon}_{y y}=$ -0.005 and $\dot{\gamma}=0.005$. The label ' $\mathrm{T}$ ' indicates the TTCF result and ' $\mathrm{D}$ ' the direct average over the same non-equilibrium trajectories. 
deformation rate, the relative importance of the initial inaccuracy becomes larger as the external field becomes smaller.

From the transient stresses and the velocity gradient, a 'transient viscosity' can be calculated. The viscosity is calculated using the expression presented by Hounkonnou et al. 207.

$$
\eta(t, \dot{\gamma}, \dot{\epsilon})=-\frac{\Pi(t): \mathbf{S}}{\mathbf{S}: \mathbf{S}},
$$

where $\boldsymbol{\Pi}=\mathbf{P}-p \mathbf{I}$ is the (traceless) viscous pressure tensor and $\mathbf{S}=\nabla \mathbf{u}+(\nabla \mathbf{u})^{\mathrm{T}}$ the symmetric strain rate tensor. Eq. 5.50 reduces for shear flow to $\eta_{s}=-\frac{P_{x y}+P_{y x}}{2 \dot{\gamma}}$ and for elongational flow to $\bar{\eta}=\frac{P_{y y}-P_{x x}}{4 \dot{\epsilon}}$. Hounkonnou et al. [207] derived this expression and replaced the viscous pressure tensor with the full pressure tensor, which in theory gives the same result. In practice, however, this is only the case in the statistical limit due to the uncertainty explained above. We use the formulation where the viscosity follows from the viscous stress. The steady-state field-dependent viscosity follows from

$$
\eta(\dot{\gamma}, \dot{\epsilon})=\lim _{t \rightarrow \infty} \eta(t, \dot{\gamma}, \dot{\epsilon})
$$

Figure 6.7 shows the viscosity for a mixed flow with $\dot{\gamma}=0.005$ and $\dot{\epsilon}=0.005$. The TTCF response clearly converges to the steady-state viscosity $\eta=2.35 \pm 0.02$, whereas the direct average (calculated from the same number of trajectories) remains noisy. The viscosity calculated from a steady-state long time-average at the same state point and with the same deformation rate is $\eta=2.31 \pm 0.09$, which is in good agreement with the TTCF result.

The results shown in Figures 6.8 and 6.9 illustrate, for a mixed flow with $\dot{\gamma}=0.001$ and $\dot{\epsilon}=0.001$, that the statistical inaccuracy of $\langle B(0)\rangle$ becomes relatively important for very small fields, where the response is small. While the response to the field at $t>0$ is very accurate for weak fields, the error in the initial $t=0$ ensemble implies an error in the trajectory origin. The figures show that the magnitude of the error bars remains approximately constant in time, meaning that the initial error in $\langle B(0)\rangle$ (at equilibrium) dominates the uncertainty for all time. The inset in Figure6.9 shows that the equilibrium value $(t=0)$ of both normal stresses are not identical. Similarly, Figure 6.8 shows that the initial shear stress is non-zero due to numerical inaccuracy. In the case of $B=P_{x y}$, we know that the initial value should be zero, and for the normal stresses we know that in the thermodynamic limit $P_{x x}(0)=P_{y y}(0)=P_{z z}(0)$ has to apply. However, a generic approach to eliminate the uncertainty of the direct averages $\langle B(0)\rangle$ is unknown. Note that the viscosity calculation does not suffer from this inaccuracy, since only the viscous stresses are taken into account. The viscosity calculated for a mixed flow with $\dot{\gamma}=0.001$ and $\dot{\epsilon}=0.001$ converges to $\eta=2.31 \pm 0.01$, which is slightly lower than the viscosity calculated for a field $\dot{\gamma}=0.005$ and $\dot{\epsilon}=0.005$. 


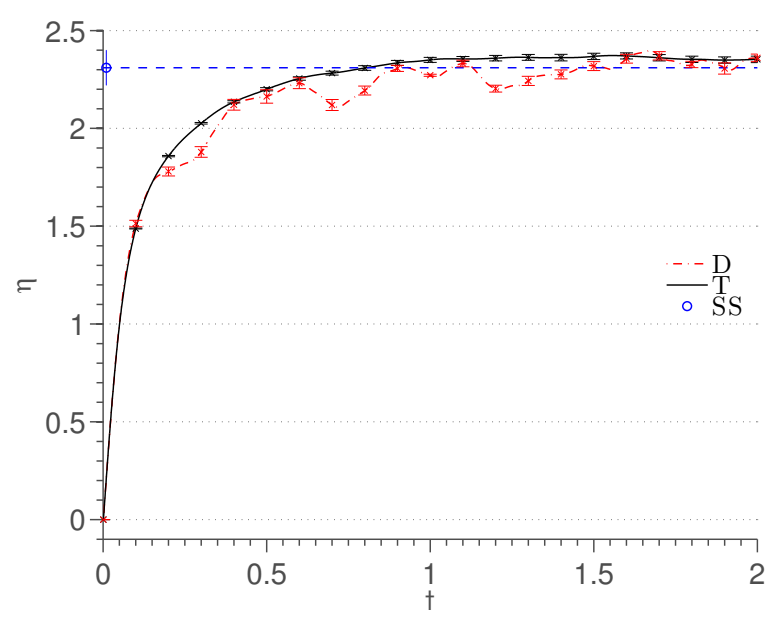

Figure 6.7: Viscosity for mixed flow with $\dot{\gamma}=0.005$ and $\dot{\epsilon}=0.005$. The label ' $\mathrm{T}$ ' indicates the TTCF result, 'D' the direct NEMD average and 'SS' is the viscosity calculated from a steady-state time-average of a different simulation at the same state point.

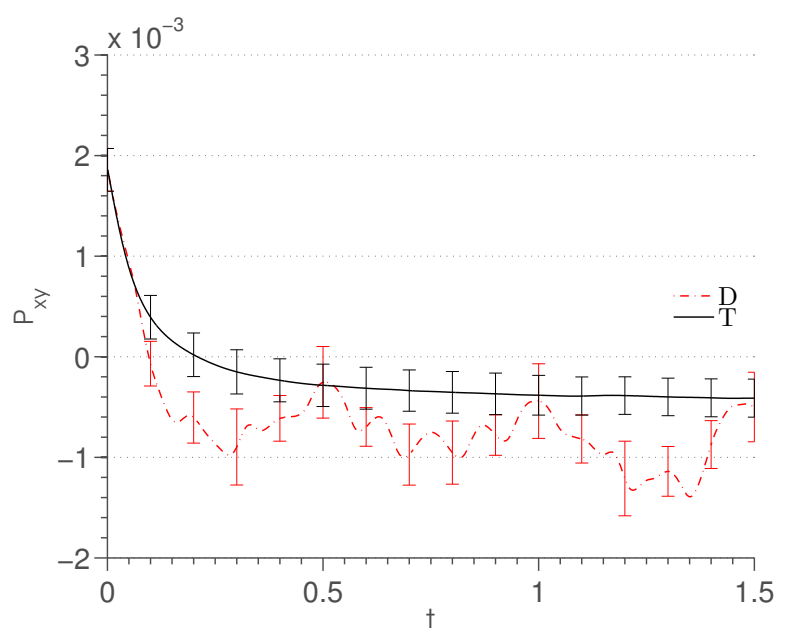

Figure 6.8: $B=P_{x y}$ for mixed flow with $\dot{\gamma}=0.001$ and $\dot{\epsilon}=0.001$. The label ' $\mathrm{T}$ ' indicates the TTCF result and ' $\mathrm{D}$ ' the direct NEMD average. The error in the starting point of the trajectory is of the same order of magnitude as the response. 


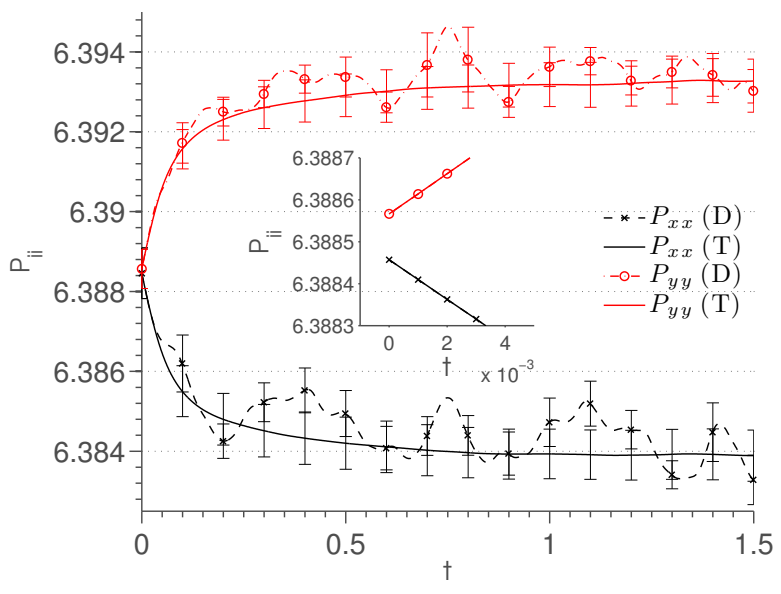

Figure 6.9: Normal stress response under planar mixed flow with $\dot{\epsilon}_{x x}=0.001, \dot{\epsilon}_{y y}=$ -0.001 and $\dot{\gamma}=0.001$. The label ' $\mathrm{T}$ ' indicates the TTCF result and ' $\mathrm{D}$ ' the direct NEMD average. The inset shows the normal stresses directly after the external field is activated. The error bars are not shown in the inset, since they are too large to fit in the domain shown.

For an even smaller field, the numerical uncertainty in the starting states of the trajectories becomes even more dominant. To illustrate the difference with the stress fields shown previously, Figures 6.10 and 6.11 show the viscous stresses for a mixed flow with $\dot{\gamma}=5 \times 10^{-4}$ and $\dot{\epsilon}=5 \times 10^{-4}$. The number of atoms is again $N=896$ and the number of trajectories used is now $10 \times 2 \times 20,000$. The viscous stresses clearly show the difference in quality between the direct averages and TTCF. The standard deviation for the direct averages is large relative to the response, whereas TTCF results in a smooth profile with a high accuracy 3 The viscosity converges to $\eta=2.28 \pm 0.01$, which is again slightly lower than the viscosity calculated for a field $\dot{\gamma}=0.001$ and $\dot{\epsilon}=0.001$. The direct averaged stress fields are much too noisy to calculate a meaningful viscosity.

For these small fields, the nonlinearity of the response of the atomic fluid becomes negligibly small and the viscosity approaches the Newtonian regime. In this regime, the transport properties are independent of the external field, thus simulations are suitable for comparison to experiments on simple Newtonian fluids.

\footnotetext{
${ }^{3}$ The TTCF and direct-averaged profile for the shear stress do not overlap. There is a $95 \%$ probability for the profiles to be within two standard errors of each other. This is not convincingly true in Figure 6.10 While the discrepancy is statistically improbable, no anomaly has been found in other sets of simulation data.
} 


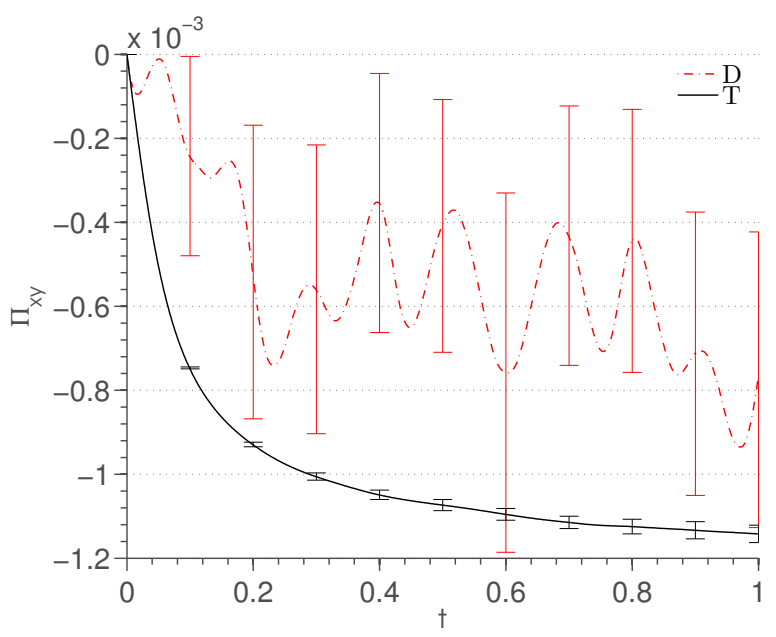

Figure 6.10: Viscous shear stress $\Pi_{x y}$ for mixed flow with $\dot{\gamma}=5 \times 10^{-4}$ and $\dot{\epsilon}=5 \times 10^{-4}$. The label ' $\mathrm{T}$ ' indicates the TTCF result and ' $\mathrm{D}$ ' the direct average over the same nonequilibrium trajectories. The number of trajectories used is $10 \times 2 \times 20,000$.

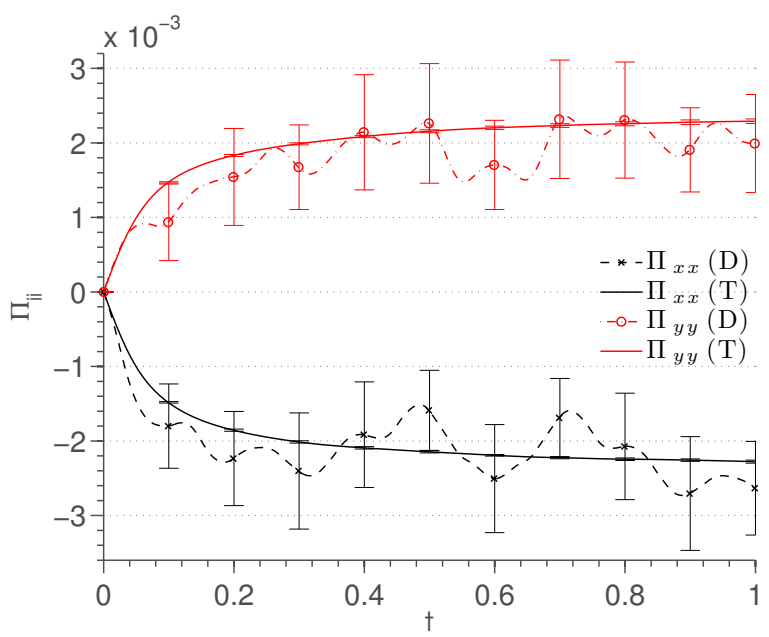

Figure 6.11: Viscous normal stress response under planar mixed flow with $\dot{\epsilon}=5 \times$ $10^{-4}$ and $\dot{\gamma}=5 \times 10^{-4}$. The label ' $\mathrm{T}$ ' indicates the TTCF result and ' $\mathrm{D}$ ' the direct average over the same non-equilibrium trajectories. The number of trajectories used is $10 \times 2 \times 20,000$. 


\subsection{Normal stress differences}

In Chapter 2 it was shown that the diagonal components of the Cartesian pressure tensor are very close to each other in a shear flow with a small shear rate. A small signal-to-noise ratio makes it computationally expensive to calculate the normal stress differences accurately from a direct average of NEMD simulation data. TTCF provides a more efficient alternative for the calculation of normal stress differences in a fluid under slow shear.

Desgranges and Delhommelle 284] used the TTCF formalism to calculate normal stress differences in a shear atomic fluid. However, their calculations were mostly limited to large shear rates, where their TTCF profiles still looked too noisy to establish their correctness. Below, the problem is revisited and a suitable phase space mapping is introduced in order to allow for accurate calculations at low shear rates.

The TTCF formulation (Eq. 6.10) can be applied to calculate the diagonal components of the pressure tensor. The statistics can be enhanced by applying phase space mappings, such as was done for the simulations performed in Section6.2. In addition to enhancing the statistics, the phase-space mappings that are commonly used for shear flow 28] are chosen such that they set the ensemble average of the equilibrium shear stress exactly to zero: $\left\langle P_{x y}(0)\right\rangle_{M}=\frac{1}{M} \sum_{M}\left\langle P_{x y}(0)\right\rangle=0$, where $M$ indicates the mappings of the starting states. Due to this feature, the transient shear stress profile starts exactly at zero, as we know to be true for a simple fluid in equilibrium. Mappings that have this property are especially beneficial when the applied shear rate is very small, such that the non-equilibrium response might be of the same order of magnitude as the statistical noise.

A suitable phase-space mapping becomes even more important for the calculation of normal stress differences, since these are often very small in slowly sheared fluids. A phase space mapping is developed to ensure that the following condition is satisfied

$$
\left\langle P_{x x}(0)\right\rangle_{M}=\left\langle P_{y y}(0)\right\rangle_{M}=\left\langle P_{z z}(0)\right\rangle_{M}
$$

A set of mappings that together satisfy this condition is given by

$$
\begin{aligned}
& \boldsymbol{\Gamma}_{1}=\left(\mathbf{x}, \mathbf{y}, \mathbf{z}, \mathbf{p}_{x}, \mathbf{p}_{y}, \mathbf{p}_{z}\right) \\
& \boldsymbol{\Gamma}_{2}=\left(\mathbf{z}, \mathbf{x}, \mathbf{y}, \mathbf{p}_{z}, \mathbf{p}_{x}, \mathbf{p}_{y}\right) \\
& \boldsymbol{\Gamma}_{3}=\left(\mathbf{y}, \mathbf{z}, \mathbf{x}, \mathbf{p}_{y}, \mathbf{p}_{z}, \mathbf{p}_{x}\right)
\end{aligned}
$$

Since this phase space mapping switches Cartesian directions with each other (without changing any of the distances between particles), the pressure tensor components of 
the mapped phase space vectors are related as

$$
\begin{gathered}
P_{x x}\left(\boldsymbol{\Gamma}_{1}, 0\right)=P_{y y}\left(\boldsymbol{\Gamma}_{2}, 0\right)=P_{z z}\left(\boldsymbol{\Gamma}_{3}, 0\right), \\
P_{y y}\left(\boldsymbol{\Gamma}_{1}, 0\right)=P_{z z}\left(\boldsymbol{\Gamma}_{2}, 0\right)=P_{x x}\left(\boldsymbol{\Gamma}_{3}, 0\right), \\
P_{z z}\left(\boldsymbol{\Gamma}_{1}, 0\right)=P_{x x}\left(\boldsymbol{\Gamma}_{2}, 0\right)=P_{y y}\left(\boldsymbol{\Gamma}_{3}, 0\right) .
\end{gathered}
$$

If we average over the mapped initial states, the diagonal components of the pressure tensor become identical

$$
\begin{aligned}
\left\langle P_{x x}(t=0)\right\rangle_{M} & =\frac{1}{3}\left(P_{x x}\left(\boldsymbol{\Gamma}_{1}\right)+P_{x x}\left(\boldsymbol{\Gamma}_{2}\right)+P_{x x}\left(\boldsymbol{\Gamma}_{3}\right)\right) \\
& =\frac{1}{3}\left(P_{y y}\left(\boldsymbol{\Gamma}_{2}\right)+P_{y y}\left(\boldsymbol{\Gamma}_{3}\right)+P_{y y}\left(\boldsymbol{\Gamma}_{1}\right)\right) \\
& =\left\langle P_{y y}(t=0)\right\rangle_{M} \\
& =\left\langle P_{z z}(t=0)\right\rangle_{M}
\end{aligned}
$$

where the subscript $M$ indicates an average over the mappings. This result ensures that the normal stress differences are exactly zero before the constant shear is imposed on the fluid in equilibrium. The TTCF formulations for normal stress differences can be written as

$$
\begin{aligned}
& \mathcal{N}_{1}(t)=\left\langle P_{y y}(t)-P_{x x}(t)\right\rangle=\dot{\gamma} \beta V \int_{0}^{t} d s\left\langle\left(P_{x x}(s)-P_{y y}(s)\right) P_{x y}(0)\right\rangle, \\
& \mathcal{N}_{2}(t)=\left\langle P_{z z}(t)-P_{y y}(t)\right\rangle=\dot{\gamma} \beta V \int_{0}^{t} d s\left\langle\left(P_{y y}(s)-P_{z z}(s)\right) P_{x y}(0)\right\rangle,
\end{aligned}
$$

where the ensemble averages of the observables before the field is activated have cancelled out exactly (i.e., the first term on the right-hand side of Eq. 6.10)).

Note that the proposed phase mapping mappings do not exactly satisfy $\left\langle P_{x y}(0)\right\rangle_{M}=$ 0 . The mappings that satisfy the condition for the initial shear stress are based on changing the signs of the position and momenta in the $x$ or $y$ direction. This causes the shear stress of the mapped phase space to have opposite signs and the same magnitude as the shear stress of the unmapped state. The sign change does not affect the TTCF response since $B(0)=P_{x y}(0)$ and $\Omega(0)=\dot{\gamma} \beta V P_{x y}(0)$ both switch their sign for the calculation of the shear stress response, such that the sign change cancels out. This is not true in case $B \neq P_{x y}$. We have not succeeded in finding a phase space mapping that satisfied this condition for shear stress, as well as the condition for the normal initial stress differences.

We have simulated a WCA fluid at a state point set to $\rho=0.84$ and $T=1.0$. The fluid is sheared at a constant rate $\dot{\gamma}=0.1$ and the equations of motion are integrated with a fourth-order Runge-Kutta scheme with a time-step of $\Delta t=0.001$. The data is averaged over $175 \times 3 \times 20,000$ trajectories. The TTCF formalism is used to calculate the diagonal components of the Cartesian pressure tensor. 


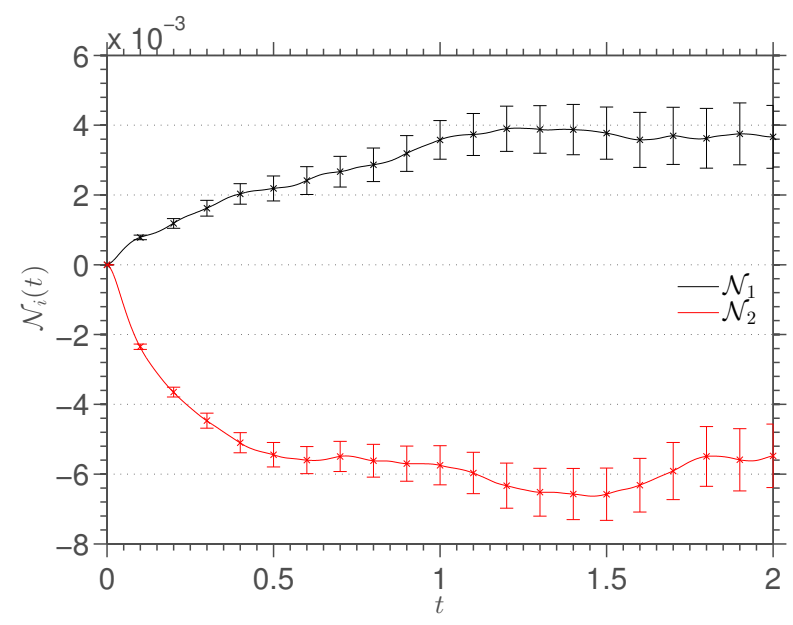

Figure 6.12: Transient normal stress differences for a WCA fluid sheared at $\dot{\gamma}=0.1$. The data is averaged over $175 \times 3 \times 20,000$ trajectories.

Figure 6.12 shows the transient normal stress differences of a sheared fluid. As expected for a shear simple fluid at this state point, the first normal stress difference is positive, while the second is negative. The first normal stress difference can be compared to the second-order fluid prediction presented $\mathcal{N}_{1}=\Psi_{1,0} \dot{\gamma}^{2}$ (see Section 5.3), where the zero shear-rate first normal stress coefficient $\Psi_{1,0}=0.418 \pm 0.006$ is calculated from the appropriate integrals over the equilibrium stress autocorrelation function (see Chapter 5). Hence, the first normal stress difference is predicted as $\mathcal{N}_{1}(\dot{\gamma}=0.1) \approx 4.18 \times 10^{-3}$, which is in good agreement with the TTCF result.

The result in Figure6.12 shows that TTCF can be applied for the calculation of normal stress differences at small deformation rates. Also, the phase space mapping proves to result in exactly zero initial (equilibrium) normal stress differences, while leaving the transient behavior unaffected. However, it must be noted that this section is primarily intended to demonstrate the possibility, rather than engaging in an indepth study of normal stress differences in sheared fluids. While TTCF provides a more efficient alternative to direct averaging at small strain rates, accurate calculations of normal stress differences are still computationally expensive. Furthermore, the timeaveraged steady-state NEMD data in Figure B.2 show that especially the magnitude of the first normal stress difference is very small for this state point. which makes it even more difficult to calculate its value with a small relative statistical uncertainty. For example, at a shear rate $\dot{\gamma}=0.1$, the shear stress is 50 times larger than the first normal stress difference. Hence, if both quantities are to be calculated with the same relative certainty (standard error), much more data is needed for the calculation of the 
first normal stress difference. The problem increases rapidly if the shear rate is made smaller, since the normal stress difference goes down with shear rate faster than the shear stress. Atomic fluids at the state point of our simulations show shear thinning for $\dot{\gamma} \geq 0.1$. Below this shear rate the shear stress scales as $P_{x y} \propto \dot{\gamma}$, while the first and second normal stress differences scale approximately as $\mathcal{N} \propto \dot{\gamma}^{2}$ according to the second-order fluid model. Simulation results indicate a more complicated relation at larger shear rates as shown in Ref. 284] and in AppendixB.

\subsection{Molecular TTCF}

The transient response of shear flow [27, 44, 277, 279] and various types of shear-free flows [143, 280] have been studied in the past predominantly for various types of atomic systems. We have seen in previous chapters that the onset of non-Newtonian behavior in atomic fluids occurs at shear rates much higher than those accessible in experiments. Non-Newtonian phenomena are known to occur at much weaker deformation rates for many molecular fluids [217, 285], which illustrates the importance of a method, such as TTCF, that can efficiently calculate the non-linear response of a fluid at small deformation rates.

Applying TTCF to a molecular fluid is not much different from what was explained in Section 6.1 for atomic fluids. The general expression is again given by

$$
\langle B(t)\rangle=\langle B(0)\rangle-\beta V \int_{0}^{t}\langle\Delta B(s) \Omega(0)\rangle d s .
$$

The initial rate of energy dissipation is now given by $\Omega(0) \equiv-\beta V \mathbf{P}^{M}(0): \nabla \mathbf{u}$, where $\mathbf{P}^{M}$ is the molecular pressure tensor, given by

$$
\mathbf{P}^{M}=\frac{1}{V}\left(\sum_{i=1}^{N_{m}} \frac{\mathbf{p}_{i} \mathbf{p}_{i}}{M_{i}}-\frac{1}{2} \sum_{i=1}^{N_{m}} \sum_{\alpha=1}^{N_{b}} \sum_{j \neq i}^{N_{m}} \sum_{\beta=1}^{N_{b}} \mathbf{r}_{i j} \mathbf{F}_{i \alpha j \beta}\right),
$$

where $M_{i}$ is the total mass of molecule $i, \mathbf{p}_{i}$ the center-of-mass peculiar momentum, $\mathbf{r}_{i j}=\mathbf{r}_{j}-\mathbf{r}_{i}$ is the separation between the center of mass of molecules $i$ and $j, \mathbf{F}_{i \alpha j \beta}$ is the intermolecular force vector acting on atom $\alpha$ in molecule $i$ due to atom $\beta$ in molecule $j$, the number of molecules is denoted by $N_{m}$ and the number of atoms in a molecule is given by $N_{b}$.

To date, studies of TTCF applied to molecular fluids are limited to a homogeneous shear flow. Pan and McCabe 281] have applied TTCF to calculate the viscosity of $n$-decane in a homogeneous shear flow, using a coarse-grained United Atom model. They have calculated the steady-state shear viscosity for reduced shear rates as low as $\dot{\gamma}=5 \times 10^{-7}\left(2.13 \times 10^{5} \mathrm{~s}^{-1}\right)$. However, their results showed a large relative 
error, even for the strain rates where a direct comparison with NEMD results was possible. Furthermore, they used an atomic thermostat and calculated the atomic pressure tensor, instead of applying the molecular formulations. The transient response of the atomic pressure tensor is different from the response of the molecular pressure tensor because the atomic and molecular initial phase space distribution functions are not identical [149]. Later, Mazyar et al. 282] extended the study of Pan and McCabe [281] with molecular pressure calculations. Furthermore, they went down to shear rates an order of magnitude smaller than in the previous work. However, their results also showed large relative errors and it is hard to say with certainty if the non-equilibrium trajectories had reached a steady state.

\subsubsection{Simulation details}

Similar to the atomic fluid simulations in Section6.2 the equations of motion are integrated with a fourth-order Runge-Kutta scheme with a time-step of $\Delta t=0.001$, in reduced units. The state point of the fluid is given by the particle density $\rho=0.84$ and temperature $T=1.0$. The temperature is kept constant with a molecular Gaussian thermostat, that controls the molecular kinetic temperature (see Section 3.1.2).

The interactions between two adjacent beads in a molecule can be modeled using the finitely extensible nonlinear elastic (FENE) dumbbell potential [286. This is an anharmonic potential that connects two adjacent beads to each other

$$
U^{F E N E}(r)= \begin{cases}-0.5 k R_{0}^{2} \ln \left(1-\left(r / R_{0}\right)^{2}\right), & r \leq R_{0} \\ \infty, & r>R_{0}\end{cases}
$$

where $R_{0}$ is the maximum length of the spring and $k$ the spring stiffness. The values for $R_{0}$ and $k$ are set to $1.5 \sigma$ and $30 \epsilon / \sigma^{2}$, respectively, as is standard in the literature [286, 287].

The FENE model does not constrain bond angles or dihedral angles, thus allowing chains to cross themselves. The WCA potential is used in addition to the FENE interactions to account for the so-called excluded volume effect. This means that the repulsive potential prohibits the beads from overlapping. The energy potential of a combined FENE and WCA potential is shown in Figure6.13. The potential energy between two particle has a minimum at distance $r_{m}=0.9608$.

The average bond length in simulations is not only dependent on the potential energy function, but is also influenced by the type of molecules and the state point of the fluid. For linear chains of lengths varying from 2 (dimers) to 10 at the state point ( $\rho=0.84, T=1.0$ ), we find average bead-to-bead distances in the range of $0.96-0.97 \sigma$, which is consistent with the findings of Hunt and Todd [287]. 


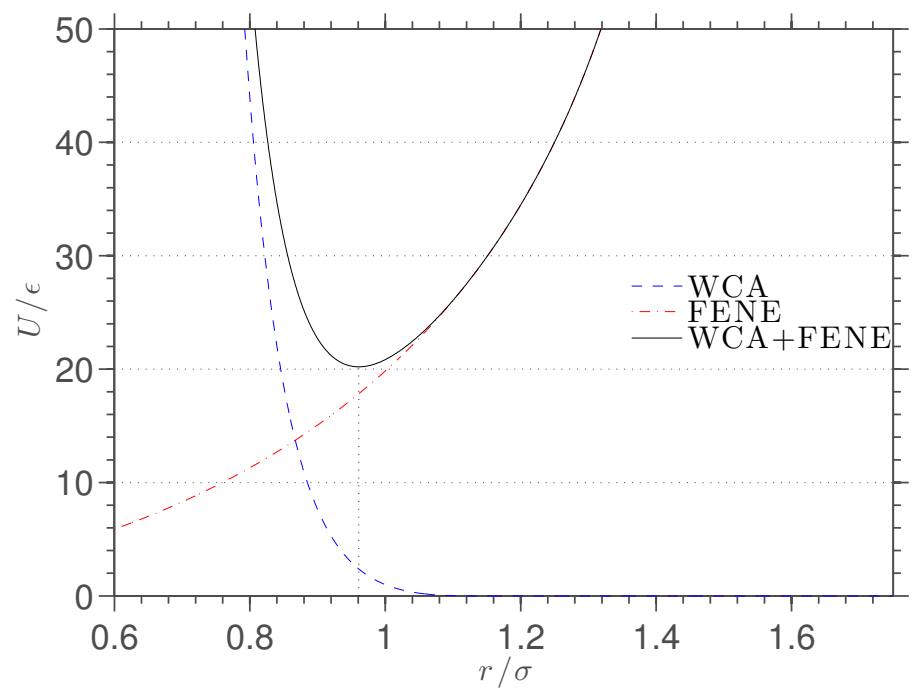

Figure 6.13: Combined FENE and WCA potential

Figure 6.14 shows two snapshots of a planar mixed flow $(\dot{\gamma}=0.1, \dot{\epsilon}=0.1)$ simulation of linear chain molecules. The length of the chains is 16 beads. Most chains are stretched due to the flow, where the chains have a preferred orientation that is approximately equal to the principal orientation of the PMF strain rate tensor $\phi=\frac{1}{2} \tan ^{-1}(\dot{\gamma} /(2 \dot{\epsilon}))$.

The periodic boundary conditions for PCF, PEF and PMF can be applied to a molecular fluid in a similar way as to atomic fluids. One can choose to apply the boundary conditions to the center-of-mass positions of the molecules or to each individual atom. The only difference between these are the implementation in the code and we have chosen the first option.

The TTCF profiles are calculated from approximately $\mathcal{O}\left(10^{5}-10^{6}\right)$ non-equilibrium trajectories. All simulations in this section contain $N=512$ particles and the number of molecules is given by $N_{m}=N / N_{b}$. Similar to the atomic TTCF calculations, time-reversal mappings are applied in order to enhance the statistics.

\subsubsection{Results}

Figure 6.15 shows TTCF viscosity profiles for a sheared fluid $(\dot{\gamma}=0.002)$ of short chain molecules. The figure shows that the steady-state viscosity increases with the chain length (or molecular mass), which is well known [286, 288]. Furthermore, the relaxation time of the fluid increases dramatically with the chain lengths. Since the steady-state 

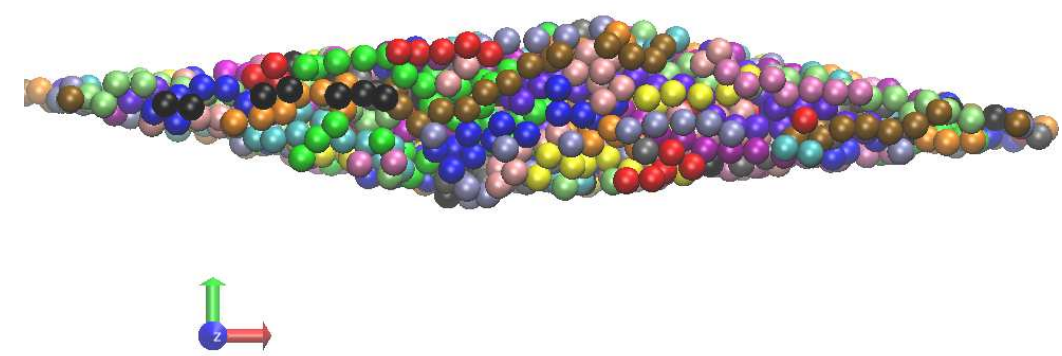

(a) Shortly before remapping of the simulation cell.

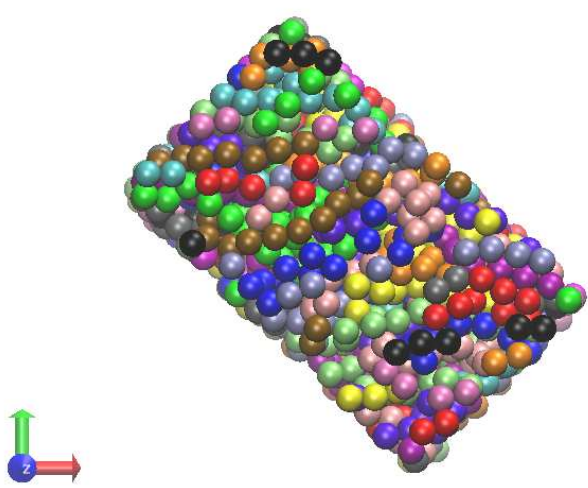

(b) Directly after remapping of the simulation cell.

Figure 6.14: Snapshots of linear chain molecules under planar mixed flow. The color coding of the atoms indicates which molecule they belong to. 


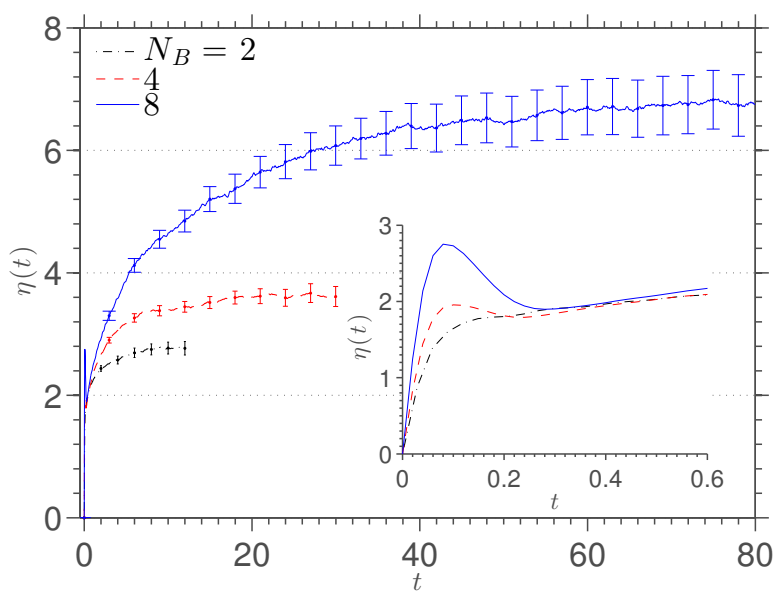

Figure 6.15: TTCF shear viscosity for short chain molecules $(\rho=0.84, T=1.0$ and $\dot{\gamma}=0.002)$.

TTCF response is calculated by integrating a correlation function up to the point where it has relaxed to zero, a longer relaxation time increases the computational cost of the TTCF calculations. The computational cost does not only increase because the correlation function needs to span a longer period of time, but also more ensembles are needed in order to reach the same accuracy for the calculated steady-state value, since the statistical uncertainty of the integrated correlation function propagates with its time length. Despite the drawback of long relaxation times, as the shear rate becomes lower, the efficiency of TTCF goes down less quickly than that of direct ensemble averaging. Hence, there will always be a shear rate below which TTCF is preferable over direct averaging. The value of this critical shear rate is related to the fluid. At short times, the TTCF profiles show a thin peak, after which the profile increases monotonically to its steady state value. The magnitude of the peaks increase with the chain lengths, as also observed by Rudisill and Cummings [271]. These authors also observed that the location of the peaks slightly moved to the right for longer chains. This observation cannot be conclusively confirmed or denied by our data. The overshoot in Figure6.15 is different from the overshoot seen in the response of an atomic fluid sheared at a large strain rate. The overshoot seen for the molecular transient viscosity profile is observed even at very small strain rates and in the integral over an equilibrium stress autocorrelation function [289]. The overshoot is more likely to be related to the structure of the molecules, as we will investigate further below. In addition to this overshoot, the effect of large strain rates can also be present in molecular fluids, as studies have shown [258, 270, 290]. 


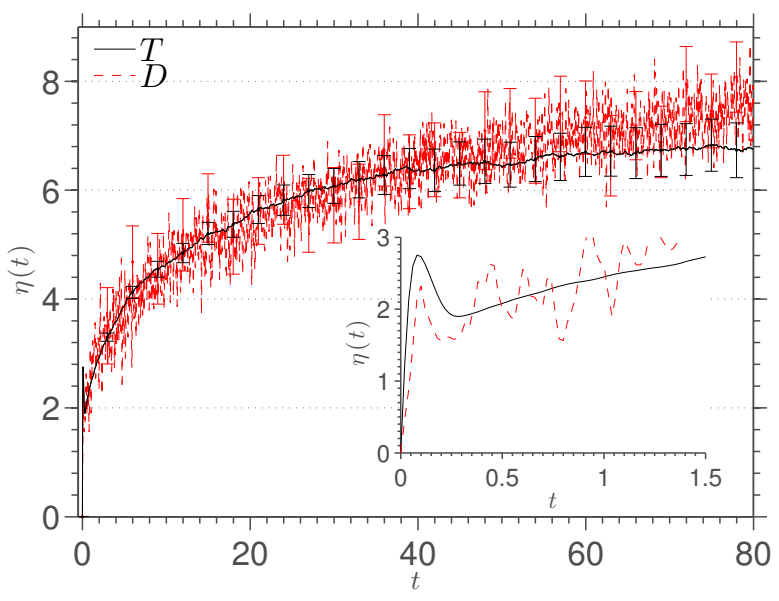

Figure 6.16: Shear flow $(\dot{\gamma}=0.002)$ of FENE chains of length 8. The direct NEMD average is indicated by ' $D$ ', whereas the TTCF result is indicated by ' $T$ '.

Figure 6.16 shows the transient viscosity of a molecular fluid with linear FENE chains of 8 beads, sheared at a strain rate $\dot{\gamma}=0.002$. The data is averaged over $100 \times 2 \times 1200=2.4 \times 10^{5}$ non-equilibrium trajectories, where the first number indicates the number of distinct simulations, the second is the number of simultaneous non-equilibrium trajectories branched off from an equilibrium state (i.e., the original equilibrium starting state and the mapped starting state) and the last number indicates how many pairs of non-equilibrium trajectories are branched off per simulation. Each trajectory corresponds to a simulation containing $N_{m}=64$ molecules. The figure shows that the direct average over the non-equilibrium trajectories results in large fluctuations and uncertainty along the whole profile, while the error bars in the TTCF profile increase with the lag time, due to propagation of uncertainty [257]. The direct average and the TTCF profile are equal to within statistical uncertainty.

In order to study the origin of the overshoot in the TTCF profile, we calculate the equilibrium SACF for a diatomic liquid. Since the FENE chains have translational, rotational and internal vibrational degrees of freedom, we can simplify the problem further by constraining the bond lengths, such that only the translational and rotational degrees of freedom remain in the correlation function. Molecules with rigidly fixed bond lengths and unconstrained bond angles and dihedral angles are called Freely Jointed Chains (FJC). The bond length in the FJC molecules is fixed by a holonomic constraint algorithm 291]. In order to compare similar molecules, we have set the FJC bond lengths to $b=0.96$, which corresponds to the average bond length of the FENE chains measured from an equilibrium simulation. 


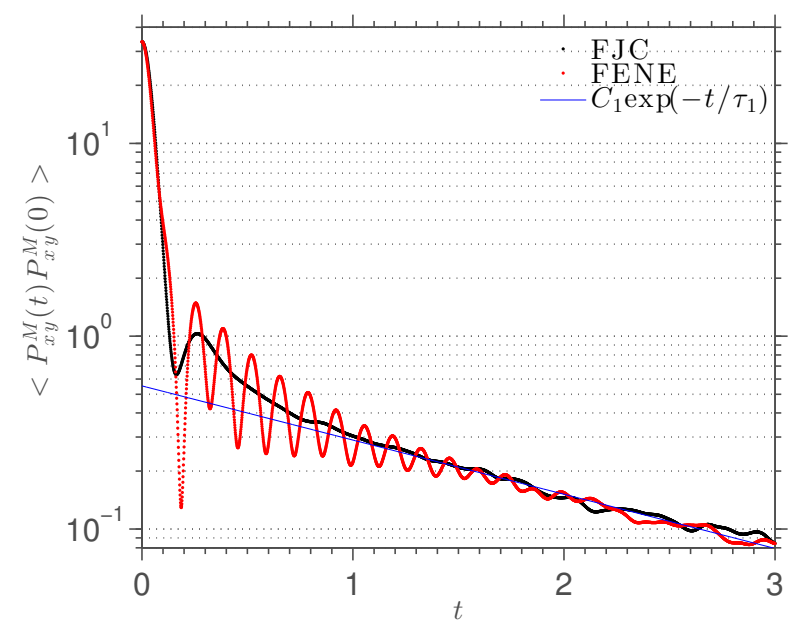

Figure 6.17: Equilibrium SACF for diatomic FJC and FENE molecules.

Figure 6.17 shows the equilibrium stress autocorrelation function (SACF) for diatomic FENE and FJC liquids. The modes of relaxation can be identified for this relatively simple system, assuming that cross-correlations between different modes are negligible. At short times, the SACF has a Gaussian-like shape. This initial shape is a property of autocorrelation functions and follows naturally from a Taylor-series expansion of the correlation function around $t=0$, as shown in Chapter 4 . Furthermore, both correlation functions in Figure 6.17 seem to decay exponentially at long times, with a relaxation time of $\tau_{1}=1.55$, as shown by the fit. This mode corresponds to the correlation between the translational degrees of freedom and appears to be the slowest relaxation mode for the short chain molecules. The deviation of the FJC correlation function from the exponential represents the correlation between the rotations of the molecules.

Rotations are often responsible for the long relaxation time in molecular fluids [289. This is however, not the case for very short chains. The rotational mode in the SACF is isolated by subtracting the exponential mode from the SACF of the FJC fluid. The remaining function is fitted (beyond the first trough) by an exponentially decaying function with a relaxation time of approximately $\tau_{2}=0.20$, as shown in Figure 6.18(a). This decay is only clearly present in a small time range, due to the dominance of statistical noise at long times, where the correlation function is predominantly described by the first exponential mode. Finally, the difference between the SACF's of the FJC and FENE fluids represents the correlation of the bond lengths. The correlation shows a oscillatory decaying behavior. This mode is shown and fitted in Figure 6.18(b) The fit is of the form $\sin (\omega t) \exp \left(-t / \tau_{3}\right)$, where $\omega=2 \pi f$ is the 


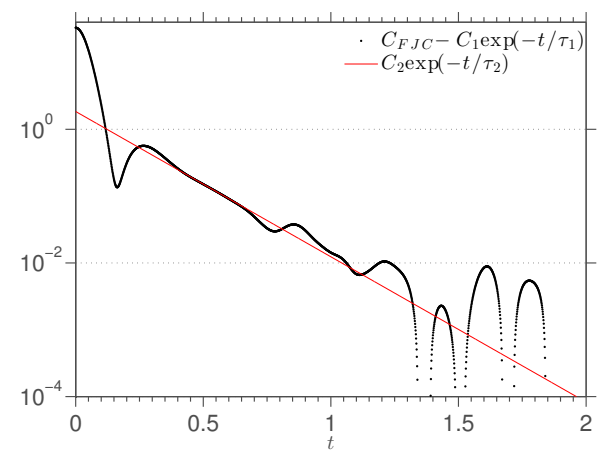

(a) The rotational mode in a diatomic FJC fluid.

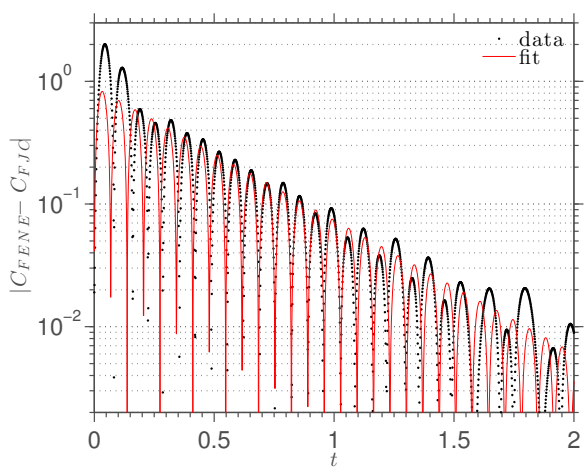

(b) The oscillations in the SACF of a FENE fluid due to bond vibrations.

Figure 6.18: Uncoupled modes of the SACF for diatomic molecules in equilibrium.

angular frequency. The relaxation time of this mode is $\tau_{3}=0.36$ and the vibrational frequency is $f=7.1$. Bond vibrations are typically the fastest mechanism in molecular fluids. Note that the period of the bond oscillations in Figure 6.18(b) is large compared to our integration time step of $\Delta t=0.001$ reduced units of time. It can be verified from the stiffness of the interaction potential that the period of oscillation is of the right order of magnitude. In this check, we used the average bond length of the FENE chains, this is approximately 0.96 (close to the minimum of the combined interaction potentials at 0.9608$)$.

The first minimum in the rotational and vibrational correlation functions results in a deep first trough in the correlation function of the FENE chain around $t=0.2$ (Figure 6.17). Longer, unconstrained chains have more internal degrees of freedom, all with their own frequencies of oscillation. Combining the correlation functions of these additional modes leads to a smoother function with a possibly negative global minimum at short times, followed by a slowly decaying positive tail. Individual modes cannot be identified anymore due to the increased smoothness of the profile.

The equilibrium correlation function of a FENE chain of 4 beads long is shown in Figure 6.19. The combined effects of the degrees of freedom of the molecules results in a negative correlation around $t=0.2$, around the same time as the first local minimum in the SACF of the diatomic liquid. This trough is followed by a positive slowly decaying correlation, where oscillatory modes can still be seen at short times. Integrating this correlation function results in a profile that is quantitatively similar to the TTCF profile shown in Figure 6.16.

Very short chain molecules are expected to respond approximately linearly at small deformation rates, where the TTCF method is advantageous. Hence, not much dif- 


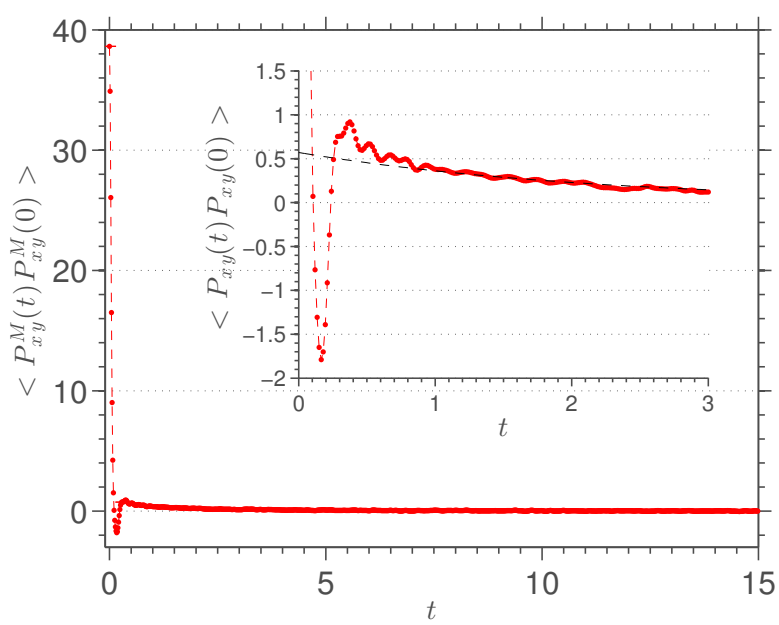

Figure 6.19: Equilibrium SACF for FENE chains of length 4.

ference is expected to be found between the Green-Kubo and TTCF results. In the remainder of this study, we present and discuss TTCF calculations for FENE chains of length 8 under shear, planar elongation and planar mixed flow.

The transient viscosity for a homogeneous flow field is calculated using Eq. 5.50). Figure 6.20 shows the transient viscosities for FENE chains of lengths 4 and 8 under simple shear, planar elongational flow and planar mixed flow. The deformation rates are chosen such that the second scalar invariant of the strain rate tensors $\left(I_{2} \equiv \mathbf{S}: \mathbf{S}=\right.$ $8.0 \times 10^{-6}$ ) are equal to each other. The deformation rates are $\dot{\gamma}=0.002$ for the simple shear, $\dot{\epsilon}=0.001$ for the planar elongational flow and the planar mixed flow corresponds to a field of $\dot{\gamma}=0.0012, \dot{\epsilon}=0.0008$. Transient viscosity profiles of monoatomic fluids are dependent only on $I_{2}$ and the state point, while the transient profiles for molecular fluids are dependent on the flow type as well. This can be easily understood since Lennard-Jones particles are considered to be spherically symmetric and rigid, whereas linear chain molecules are modeled as flexible and they have a preferred orientation under influence of a flow field. When vorticity is present, molecules tend to have a non-zero average rotational velocity. This rotation typically corresponds to the longest relaxation time in the fluid. Figure 6.20(a) shows that the transient viscosity of chains of length 4 is not visibly dependent on the type of flow. While small differences can be seen, the profiles are equal within the error bars. This implies that for the short chains, dynamical phenomena such as rotation or stretching does not have a major effect on the generalized fluid viscosity. When the chains are twice as long, the viscosity shows a clearly different dependence on the flow field (Figure6.20(b). The viscosity profile that corresponds to elongational flow is now clearly higher than that of shear flow. 


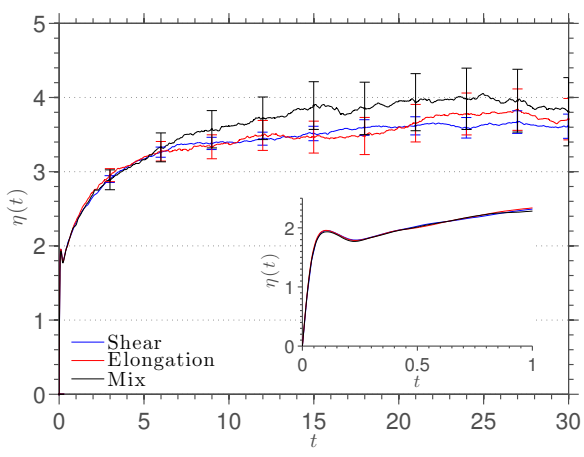

(a)

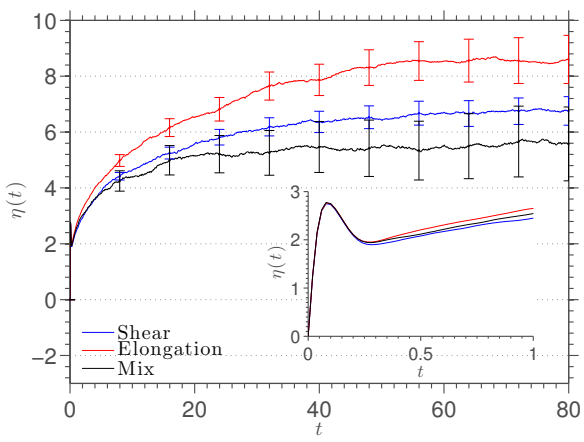

(b)

Figure 6.20: Transient viscosities for FENE chains of length 4 (a) and 8 (b) under simple shear $(\dot{\gamma}=0.002)$, planar elongation $(\dot{\epsilon}=0.001)$ and planar mixed flow $(\dot{\gamma}=$ $0.0012, \dot{\epsilon}=0.0008)$.

The mixed flow and shear flow profiles are within the error bars of each other, which implies that the elongational component in the mixed flow $8 \dot{\epsilon}^{2}=2 \dot{\gamma}^{2}=I_{2} / 2$ is too small to stretch the short chains, or similarly, the contribution of shear is large enough to prevent stretching of the chains by subjecting them to a rotational field. Due to the relatively small fields and short chains, the differences in the dynamics cannot be observed from snapshots of the simulation; it only shows up in ensemble-averaged quantities. Longer chains and a study of the gyration tensor would provide more insight in the behavior of the chains.

\subsection{Summary}

We have applied the transient-time correlation function (TTCF) to study three problems: Firstly, the viscosity of an atomic fluid under planar mixed flow. Secondly, the normal stress differences in an atomic shear flow. Finally, TTCF was applied to calculate the viscosities of molecular fluids under shear flow, planar elongational flow and planar mixed flow.

We have presented the stress response and viscosities both in the shear-thinning region and in the Newtonian region. Good agreement was found between direct averages of NEMD simulations and the TTCF response for relatively large field strengths. For small field strengths, the direct averages show a decrease in the accuracy of the calculation, whereas the accuracy in the TTCF response is invariant to changes in the field strength. TTCF proves to be far more efficient at small deformation rates than 
direct averages of NEMD simulations. Therefore, this method can be applied to fluids with deformation rates which are much smaller than those commonly used in NEMD simulations and thus approach the field strengths that are typical in experiments.

We have shown that by subtracting the known error from the correlation function, special phase-space mappings are not required. Without the need for phase-space mappings, it becomes possible to apply TTCF, with a high accuracy, to each type of homogeneous flow that can be simulated for an indefinitely long time, for example elliptical flows. This is merely one of the yet unexplored applications for TTCF.

Normal stress effects in sheared fluids under small shear rates are extremely difficult to calculate accurately by directly averaging data from NEMD simulation. The values of the diagonal components of the pressure tensor are extremely close to each other when an atomic fluid is sheared at a small rate. Hence, very long time-averages are needed to reduce the statistical uncertainty sufficiently to be small compared to the calculated normal stress difference. Close to equilibrium, TTCF goes down in efficiency less than direct NEMD averaging does, and thus provides a suitable alternative for the calculation of normal stress differences. An atomic fluid simulation was performed and a phase space mapping was proposed to enhance the statistics of the TTCF calculation of normal stress differences in a sheared fluid. The presented results showed that TTCF in conjunction with the proposed phase space mapping can be used successfully to calculate the normal stress differences. Note that the purpose of this study was not to present extensive calculations, but merely to investigate the challenges and advantages of TTCF as an alternative method for problems where direct NEMD averaging can become practically unfeasible.

Furthermore, we have shown that the transient-time correlation function can also be used to calculate steady-state properties of a molecular fluid under shear, elongational and mixed flows. We have studied the transient response of linear chain molecules consisting of 2, 4 and 8 beads. We have analyzed the relaxation modes in the equilibrium stress autocorrelation functions for a dense diatomic molecular fluid. This has lead to new insights in the shape of the relaxation function and the characteristic relaxation times and frequencies of the degrees of freedom present in the fluid.

The data confirmed the well-known fact that the relaxation time of a fluid increases dramatically with increasing chain lengths. With the increase of the relaxation time, the computational time of the simulations required for the TTCF calculation goes up even more. Nevertheless, at small deformation rates, the advantage of TTCF over direct averaging of NEMD simulation results remains. We have shown that, for the deformation rates and chain lengths considered in this work, the TTCF method results calculated the transient viscosity profiles with less statistical noise than the directly 
averaged NEMD data. If we decrease the deformation rate to values that are more interesting from an industrial perspective, the efficiency of the direct averaging goes down faster than that of TTCF. Nevertheless, the computational cost of both methods increases with a decreasing deformation rate. This is the reason that we have not yet attempted to perform molecular TTCF calculations at lower rates than those presented here.

In conclusion, we have investigated TTCF as an alternative to direct ensemble averaging of data from atomic and molecular NEMD simulations with various types of flow. We have also studied phase space mappings in order to enhance the statistics of the TTCF calculations. A mapping has been engineered for the calculation of normal stress differences in shear flow, while for planar mixed only the time-reversal mapping is found to be compatible with the periodic boundary conditions. Viscosities and components of the pressure tensor have been successfully calculated with the TTCF method in each of the test cases. 


\section{Confined atomic fluids}

Thø previous chapters were concerned with systems in which spatial homogeneity could be assumed. Consequently, volume averaging was permitted as the values of macroscopic quantities were independent of the location in the system. As discussed in Chapter 1, the behavior of fluids confined in a geometry that has a characteristic length in the micrometer or nanometer range deviates from that of bulk fluid or a fluid in a much larger confinement. In this chapter, a LJ fluid is confined in one direction between two perpendicular infinitely large atomically flat walls, while the system is periodic in the other directions. The walls are formed by LJ particles arranged in a regular face-centered cubic (fcc) lattice. The wall atoms are either fixed at their lattice sites (corresponding to a zero temperature) or thetered around their respective lattice sites. The atoms near the walls of the channel tend to arrange in layers parallel to the surface, where atoms find a position that corresponds to a lower potential energy relative to the direct surrounding. This layering results in a spatially inhomogeneous distribution of atoms such that the state point of the fluid and the constitutive relations, that relate the thermodynamic force to their conjugate flux, might become a complicated function of the position [34, 292, 294.

Computer simulation studies [295-310] and experiments [12, 13, 311 313] of fluids confined in narrow channels or pores show variations in density, pressure and other quantities in the direction perpendicular to the walls. These interfacial effects generally damp out over a range of a few atomic length scales.

Nanoscale systems have an enormous surface-to-volume ratio compared to larger

This chapter is based on:

R. Hartkamp, A. Ghosh, T. Weinhart, and S. Luding (2012), J. Chem. Phys. 137, 044711,

R. Hartkamp and S. Luding (2010), Fifth International Conference on Multiscale Materials Modeling. Freiburg, Germany,

R. Hartkamp and S. Luding (2010), International Conference on Multiphase Flow. Tampa, Florida, R. Hartkamp, A. Ghosh and S. Luding (2010), World Congress Particle Technology 6, Nuremberg, 4 pages, CD-Proceedings. 

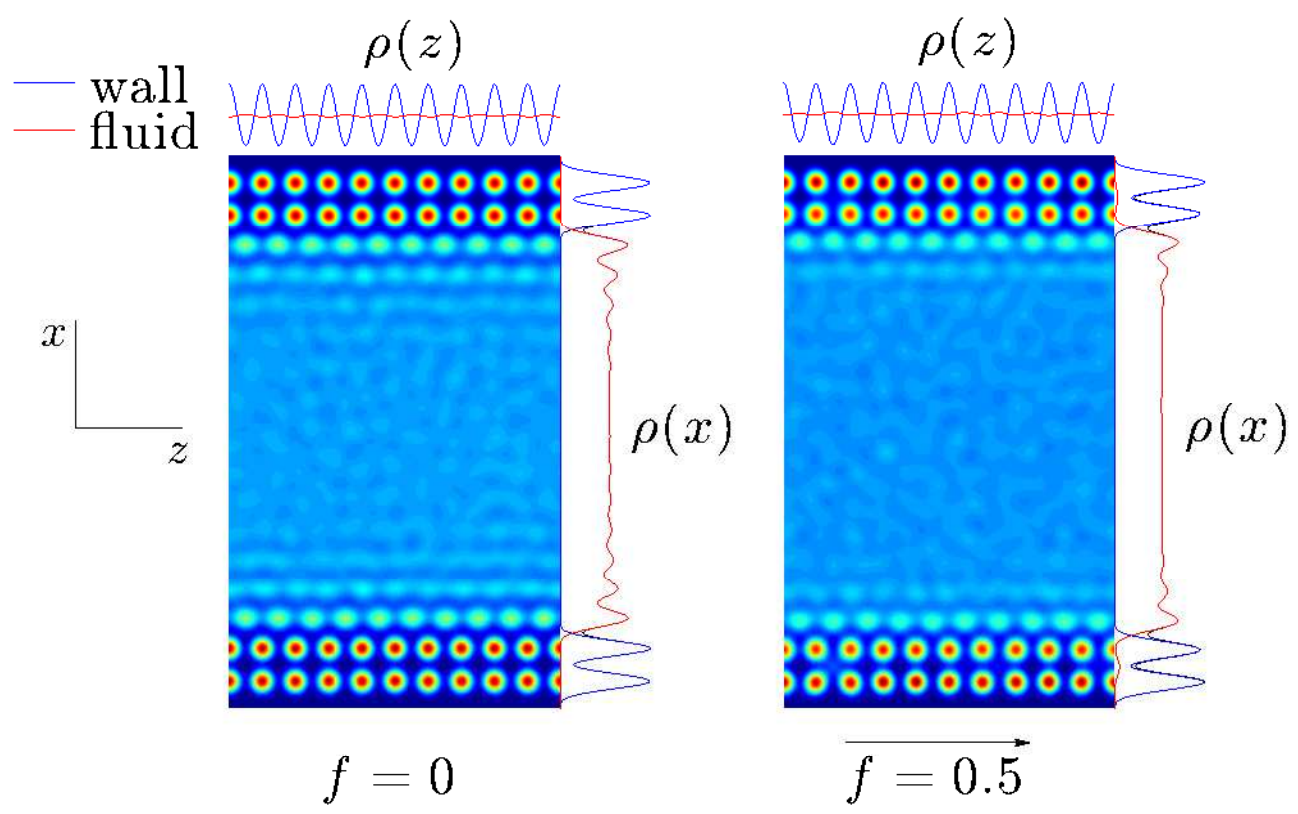

Figure 7.1: Time-averaged and spatially smoothed (with a smoothing width $h=0.6$ ) density in the direction of the flow and the direction perpendicular to the walls.

systems. Therefore, it is of paramount importance to the development of nanotechnological applications to understand the fluid behavior near solid-liquid interfaces. To study these systems with MD, atomistic walls can be explicitly included in the model, such that the effect of properties like: shape, density, roughness and hydrophilicity/hydrophobicity of the wall can be studied in a straight-forward manner. Alternatively, a wall potential can be developed to represent these properties.

Local quantities in a steady state system are generally averaged over time and over the directions parallel to the walls, assuming spatial homogeneity in these directions. While this assumption is true far enough from the walls, the validity of this assumption may be debated near the interface. Figure 7.1 shows an example of the two-dimensional density distribution of a strongly confined fluid. The data is averaged over time and over the third spatial direction. Furthermore, the information is smoothed using a two-dimensional Lucy kernel (see Section 7.3). The left side of the figure shows the density of a fluid in equilibrium, whereas the right corresponds to a fluid driven by a body force $f=0.5$. The walls are created of tethered atoms in a body-centered cubic (bcc) lattice. Both systems show a fluid density that is inhomogeneous in the 


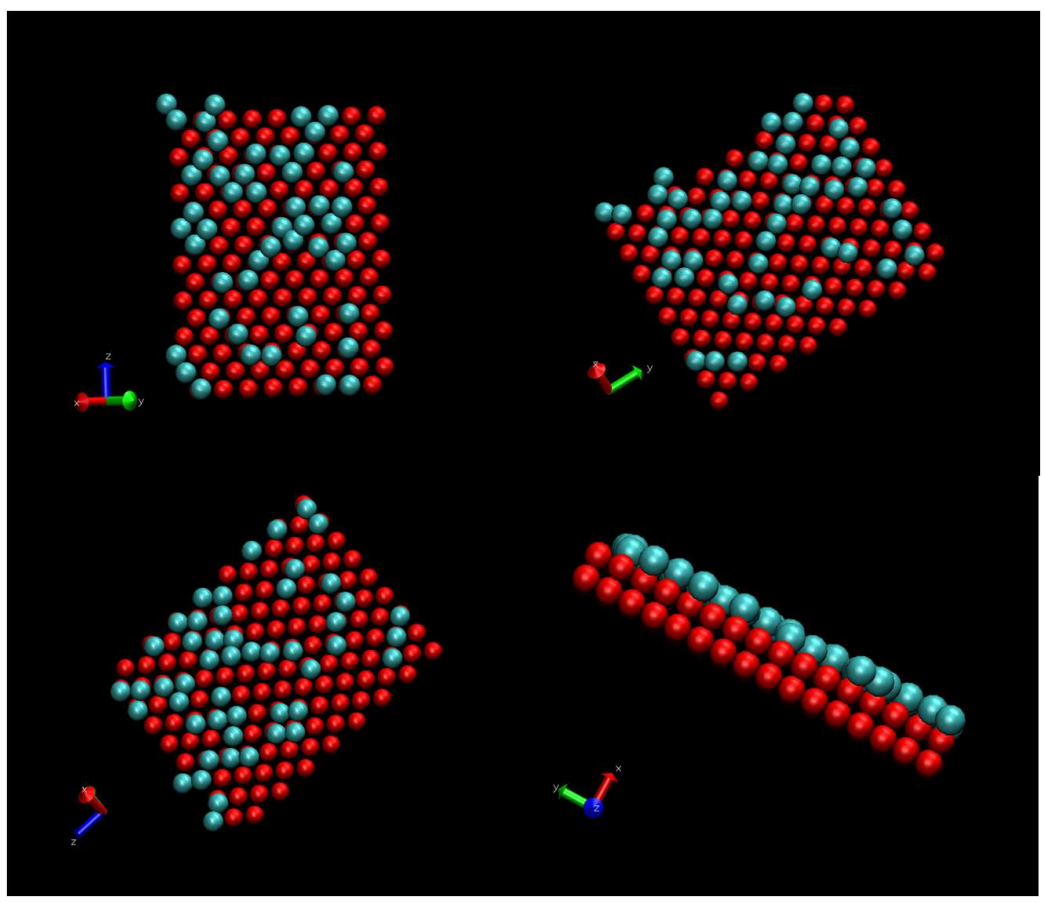

Figure 7.2: Fluid atoms (cyan) at their potential energy minimum near a solid wall (red) in a regular fixed lattice.

$x$-direction (perpendicular to the walls) and approximately homogeneous in $z$, parallel to the walls. Only very close to the walls order can be seen in the $z$-direction. This happens in both cases, but is more pronounced in equilibrium. Near the wall, atoms form triangles in the $x-z$ plane, since these locations correspond to a minimum potential energy. This is illustrated in Figure 7.2 for atoms near a fixed regular wall in a (100) face-centered cubic (fcc) lattice, the fluid is driven by a body force $f=0.1$ in the $z$-direction. The atoms very close to the lattice escape their local energy minimum, but spend slightly longer at these locations compared to positions near the wall where the potential energy is higher.

It must be noted that while atoms spend a more than average amount of time near their potential minima, the fluid atoms in the driven case are not trapped at these positions. This can be confirmed by looking at the two-dimensional streaming velocity profile of the fluid. Figure 7.3 shows the magnitudes of the velocities, i.e., the speed of the atoms as a function of $x$ and $z$. Red corresponds to high values, whereas dark blue corresponds to small velocities ${ }^{1}$ For the therered wall particles, the absolute

\footnotetext{
${ }^{1}$ Since the velocities are obtained from dividing the momentum distribution by the density distri-
} 


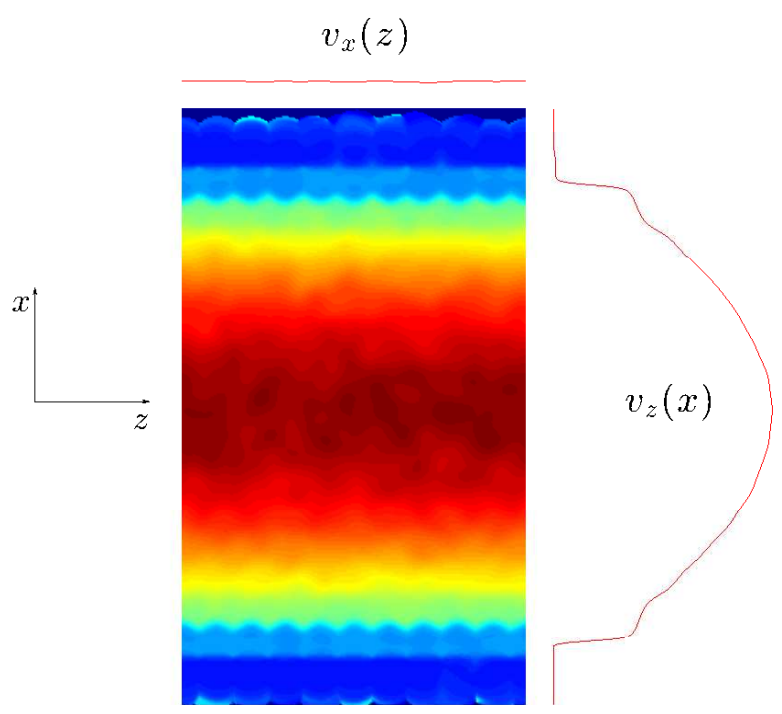

Figure 7.3: Time-averaged and spatially smoothed speed in a planar Poiseuille flow.

values of velocity are related to the temperature, since there is no streaming velocity. The figure shows that the wall layers closest to the fluid are warmer than the layers on the outside of the channel. The color profile in the fluid region shows that the distribution of velocity is independent of $z$, also very close to the walls. Furthermore, the one-dimensional smoothed streaming velocity profile on the right shows that slip occurs between the fluid and the thermal walls. The non-zero streaming velocity near the wall, in conjunction with the homogeneous distribution of velocity along the $x$ direction, indicate that atoms are not trapped at potential minima near the wall. From Figures 7.1 and 7.3 can be concluded that the fluid is, by approximation, homogeneous in the directions parallel to the walls. This assumption is made henceforth. Only profiles of a single spatial coordinate are studied in the rest of this chapter.

In addition to the conclusion that particles are not trapped at their potential energy minima very close to the walls, we can also demonstrate that particles are not trapped in any of the dense layers. Figure 7.4 shows how the $x$ coordinate of a number of atoms in a very narrow channel advances in time. The fluid density profile is also shown. The system consist of a Lennard-Jones fluid with an average density of $\rho=0.8442$ and temperature $T=0.722$ (controlled via the walls), in reduced units. The trajectories shown in the figure correspond to atoms that skip to another layer within the plotted time frame. Clearly, the exchange of atoms between the layers in the middle, which

bution, the top and bottom of the figure do not look perfectly smooth in the walls, where the density locally reaches zero. 


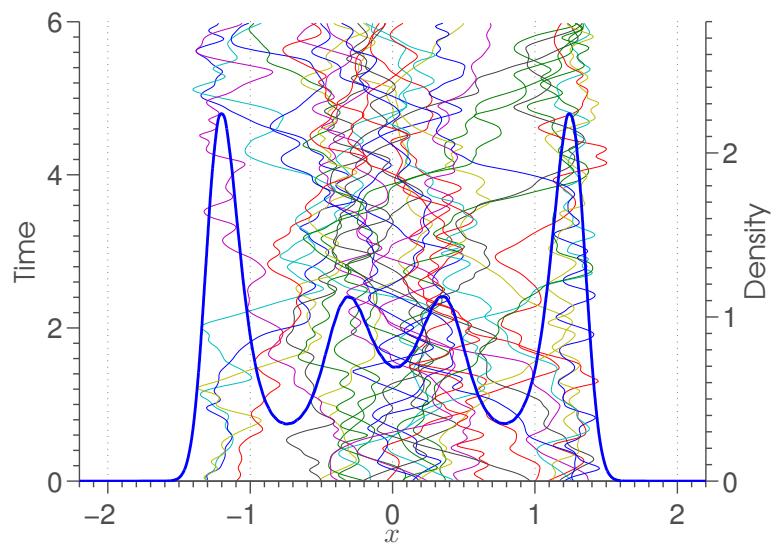

Figure 7.4: The trajectories of a few atoms in a dense fluid in a very narrow channel.

are less pronounced, occurs more often than a jump to or from the layer nearest to the wall. The fluid in this simulation is driven by a body force $f=0.2$ perpendicular to $x$. We have also confirmed with simulations that even without the body force, particles have enough kinetic energy to overcome the potential energy barrier between dense layers of fluid.

When the fluid is flowing, heat is created and transported to the wall (since the wall is colder than the fluid). Comparing the wall density in both images in Figure 7.1, it can be seen that the positions of the inner layer of wall particles is slightly more distributed over the $x$-direction when the fluid transfers more heat to the wall. However, this effect is small when the body force is small. The right image shows a non-zero fluid density in the wall, which illustrates that fluid particles can sometimes penetrate the wall. The chance of this happening increases with the body force on the fluid, since the particles will have more energy in that case, which is needed to overcome the potential energy barrier towards the wall. Once a particle has penetrated the wall, it will transfer part of its energy to the wall and often have not enough energy to leave the wall. It can sometimes move through the wall though, depending on the structure of the wall. There is a number of ways to avoid this penetration from happening. The parameters of the interaction potential between fluid and wall particles can be chosen such that the energy barrier increases. The spring stiffness of the tethers can be increased 2 An additional repulsive potential can be added between the fluid particles and an imaginary plane at the location of the wall. Furthermore, the lattice packing of the walls can be increased by changing the spacing or the lattice type. Also fixed

\footnotetext{
${ }^{2}$ Since the target temperature of the walls remains unchanged, a larger spring stiffness leads to the same vibrational velocities, but smaller amplitudes and higher frequencies.
} 
regular walls are harder to penetrate, since an impacting particle cannot push the wall particles away and thus keep the repulsive forces relatively small. This difference between tethered and fixed walls has a small effect on the density, but can have a large effect on slip between the fluid and the wall. The fact that slip becomes important on molecular length scales is another deviation from continuum theory. Slip is briefly discussed in Section 7.2. However, for a more complete treatment, the reader is referred to more specific literature [314 316].

This chapter starts with an overview of the literature in Section 7.1. Considerations regarding simulating confined fluids are discussed in Section7.2. In Section7.3, methods are discussed to calculate macroscopic fields from microscopic data. A detailed study of confined argon is presented in Section 7.4, and in Section 7.5, the conclusions are summarized.

\section{$7.1 \quad$ Literature}

Various simulations [34, 79, 317, 322] and experiments [14 16, 323, 324, have been performed on confined fluids with the aim to understand and describe the flow behavior of the system by looking at relevant global and local physical quantities. While some experiments could predict the effective global properties like relaxation time, frictional force or shear response of ultra-thin films, the extraction of local values of state variables (like density, pressure and temperature) is still beyond the reach of experimental measurements. On the other hand, such local quantities can be extracted rather easily from simulations. Several numerical studies in the past years have been devoted to gain understanding of the properties of dense fluids in a nanochannel. For example, Sofos et al. 300. performed a thorough study of the density, velocity and temperature profiles of a simple liquid in channels of several widths, temperatures, body forces and average fluid densities. One of their findings is that, while a dense fluid becomes homogeneous in the center of a wide channel, a fluid with low average density remains inhomogeneous, due to wall-effects. Recently, Long et al. 305] studied the influence of the confinement on the normal and tangential stresses for argon in a carbon nanochannel. They found that the normal stresses can be positive or negative, depending on the channel width. Furthermore, they observed that the shear stress is very sensitive to changes in the bulk pressure.

While strongly confined fluids have been widely studied, finding a constitutive relation that holds near the walls as well as in the bulk is still an open problem. The strain rate profile shows stronger oscillations than the shear stress in the region near the walls. Hence, the ratio between the shear stress and strain rate depends on the distance to the walls and is an unsuitable measure for the shear viscosity. Since 
a tensorial viscosity would increase complexity enormously, a more commonly used believe is that the shear stress relates to the strain rate via a convolution integral over a non-local viscosity kernel [34, 35, 325]. The concept of a non-local viscosity was introduced by Bitsanis et al. [34] and applied to a confined Lennard-Jones fluid. First, the local average density at any point is obtained by averaging the local density over a spherical volume centered around the point. The functional dependence of shear viscosity on density at a given temperature was then expressed using the Enskog theory of hard-sphere fluids. Their work is one of the pioneer studies that addresses the inhomogeneity in strongly confined fluids. However, it must be noted that the hardsphere theory is not strictly applicable to the Lennard-Jones fluid and the volume over which the density is averaged is not well-defined [326]. Building further on the method developed by Bitsanis et al., Hoang and Galliero 292] recently presented a study using a sinusoidally varying external potential to study the non-local viscosity of a simple fluid in a periodic box. Effective viscosities obtained by numerically integrating such local functionals over the entire domain of variation are shown to be in agreement with the value calculated from molecular dynamics simulation in different flow situations. A number of papers [79, 86, 327, 328] in the last years showed local viscosity calculations from shear stress - strain rate relations as a function of location. For example, Todd et al. 293, Cadusch et al. 329] and Todd and Hansen 294] compared local and non-local constitutive relations in narrow rectangular channels with Weeks-Chandler-Andersen (WCA) atoms [7].

Recently, Sofos et al. 298] and Sun et al. 330] have applied the Green-Kubo relation locally in order to find how the transport properties are affected by the confinement of a fluid. Sofos et al. 301] studied the influence of wall roughness on the average and local shear viscosity and diffusion coefficient. Due to a coarse bin averaging, the layering of atoms near the walls is not explicitly visible in their results. Also, their stress calculation assumes a homogeneous density across each bins, which would only be approximately satisfied far from the walls. However, a global impression of the shear stress, strain rate and shear viscosity is given across a planar channel.

Travis and Gubbins [79] studied planar Poiseuille flow in much narrower slits of pore width $4.0 \sigma$ and $5.1 \sigma$. They also use the mesoscopic integration of the Navier-Stokes equation to compute shear stress, whereas strain rates are derived from a polynomial function obtained by fitting the streaming velocity profile across the channel. The same system has been studied with different interatomic interactions (Lennard-Jones and WCA potential) to probe the effect of these interactions on the flow properties. It was found that the layering of a Lennard-Jones fluid is stronger than that of a WCA fluid with the same temperature and density. Highly nonlinear shear stress and strain rate profiles were observed across the channel irrespective of the kind of interaction 
potential used.

Jabbarzadeh et al. 317] performed a thorough study of Lennard-Jones fluids confined between atomistic walls in which the atoms are connected to their face centered cubic lattice by harmonic springs. The authors studied the influence of the channel width, shear rate and the interaction strength between fluid and wall atoms on several quantities, among which: shear viscosity, slip length, density and temperature. It was found that the shear viscosity in the channel increased with a decreasing channel width. As the channel becomes wider, the shear viscosity approaches the value the corresponds to a bulk fluid. Furthermore, they found that the strength of the fluid-wall interaction has a strong influence on density, viscosity and velocity profiles as well as on the degree of slip between the fluid and walls. A larger interaction strength leads to lower peaks in the density profile and a reduction in the slip, shear viscosity and normal stress differences.

Eslami and Mehdipour 331] simulated an argon in nanochannels of various widths. The authors studied, for a fluid at equilibrium, the density, pressure tensor and chemical potential as a function of the distance to the wall. They used a barostat to fix the average pressure tangential to the wall, while the pressure normal to the wall was not controlled. The tangential pressure showed variations in anti-phase with those in the density profiles, and the normal pressure was almost constant across the channel, as prescribed by the balance of linear momentum. The channels of width $W \geq 3.05 \mathrm{~nm}$ $(9.0 \sigma)$ showed a different behavior from the much narrower channel $(W \geq 0.845 \mathrm{~nm}$, $2.5 \sigma)$. The values of the tangential and normal pressures showed a large difference in the narrow channel. In the wider channel, both pressure components approached, towards the center of the channel, to the value that is controlled by the barostat.

Yang and Ding 332] used a nonlocal density function theory approach to study the density profile of a Lennard-Jones fluid in a square pore with planar atomistic walls. The authors found that a change in the chemical potentials or channel size had an influence on the density profile. However, all profiles showed distinct peaks in the corners of the square profile.

Very recently, Rickman [333] studied the local elastic properties of a Lennard-Jones fluid confined between two static structureless walls. He found that the local elastic properties were correlated with the variations in density near the wall and with the strength of the fluid-wall interactions.

\subsection{Simulating atomistic channels}

When studying confined fluids with MD, it must be appreciated that the results are dependent on various simulation parameters. Flow properties of monoatomic homo- 
geneous non-equilibrium fluids are mostly determined by the equations of motion in conjunction with the driving force on the fluid and the interaction potential, thermostat and the state point of the fluid. Furthermore, the properties of strongly confined fluids are a function of the location within the confinement. The problem is immensely complicated due to a large variety of parameters that affect the properties of the confined fluids. Some examples are: wall density and roughness [299, 334 336], wall lattice and orientation [337], interaction potential between fluid particles as well as between fluid and wall [79], state point of the fluid [300, 307, 338], channel size and shape [296, 339, method of temperature control [49, 104, 340, and the assumptions that are made to simplify the problem (e.g., assuming homogeneity in certain directions). Some of these considerations are discussed in this section.

It is well-known that the phase diagram of a confined fluid not only depends on the interaction potential, but also the confinement [312, 339 342. Kaneko et al. 341] studied how the phase diagram depends on the channel width for Lennard-Jones atoms confined in narrow channels (of widths: $2 \sigma-6 \sigma$ ), where the atoms form a regular lattice in frozen state. They showed that the freezing and melting points of the fluid are discontinuous functions of the channel width. This is related to the number of layers and the type of lattice of the solidified fluid. Soong et al. [337] showed the influence of the type of wall lattice on the flow properties.

Regardless of the density or structure of the wall, the wall particles are typically connected to their respective sites via harmonic springs 37

$$
U_{\text {harm }}\left(\left|\mathbf{r}_{i}-\mathbf{r}_{i, e q}\right|\right)=\frac{1}{2} k\left(\mathbf{r}_{i}-\mathbf{r}_{i, e q}\right)^{2},
$$

where $\mathbf{r}_{i, e q}$ is the position of the lattice site of an atom and $k$ is the spring stiffness. The stiffness of the spring is often chosen to be $k=57.15 \epsilon / \sigma^{2}$, which is related to the stiffness of the LJ and WCA potential at a distance $r=2^{1 / 6} \sigma$. The force due to the springs is given by the negative gradient of Eq. 7.1.

The dynamics of the fluid atoms under the influence of a body force are described by Newton's second law of motion. The equations of motion are given by

$$
\begin{aligned}
\dot{\mathbf{r}}_{i} & =\mathbf{v}_{i}, \\
\dot{\mathbf{v}}_{i} & =\frac{1}{m_{i}}\left(\mathbf{F}_{i}+\mathbf{F}^{E}\right),
\end{aligned}
$$

where $\mathbf{F}^{E}$ is an external body force vector acting on the fluid and $\mathbf{F}_{i}$ the force on atom $i$ due to surrounding atoms. The particle velocities are now expressed in the laboratory frame, in contrast to the homogeneous equations of motion in previous chapters, where the velocities relative to the streaming motion were used.

If shear is present in the fluid, heat is dissipated, which leads to an increase in temperature. A thermostat needs to be coupled to the system to remove the excess 
heat. The most natural way to do this is to thermostat the walls, as also is the case in most experimental situations. Yet, many studies deviate from this approach and couple a thermostat directly to the fluid. If the fluid is thermostatted instead of the walls, the thermal velocities are still needed to describe the dynamics of the fluid atoms. The evolution of the velocity of the fluid atoms is then given by

$$
\dot{\mathbf{v}}_{i}=\frac{1}{m_{i}}\left(\mathbf{F}_{i}+\mathbf{F}^{E}\right)-\zeta\left(\mathbf{v}_{i}-\mathbf{u}\left(\mathbf{r}_{i}\right)\right)
$$

where $\zeta$ is a thermostat multiplier. Alternatively, the fluid could be thermostatted with a configurational thermostat, as presented in Section 2.5.3. In that case, the evolution of the position of the atoms gains a term associated with the thermostat.

A simple analysis can be made to increase understanding of viscous heating in confined fluids. Energy is dissipated at a rate given by

$$
\dot{E}_{v}=-\int \mathbf{P}(\mathbf{r}): \nabla \mathbf{u}(\mathbf{r}) d V=A \int_{0}^{W} \eta(x) \dot{\gamma}(x)^{2} d x,
$$

where where $W$ is the width of the channel, $A$ is its cross section area perpendicular to the direction of the flow. The last equality used the assumption that the fluid is homogeneous in the $y$ and $z$-directions, as discussed in the beginning of this chapter. At least two important conclusions can be extracted from Eq. (7.5). In the first place, most heat is created where the velocity gradient is large. This fact distinguishes shear flow from Poiseuille flow. In shear flow, the generation of heat is not strongly dependent on the position in the channel, since the velocity profile is linear across the channel, whereas in a Poiseuille flow, the velocity profile is approximately quadratic, thus the generation of heat increases quadratically with the distance from the middle of the channel. Hence, for Poiseuille flow, most viscous dissipation takes place in the region near the walls. Second, the amount of heat that is created in the fluid scales non-linearly with the channel width. The transport of heat towards the walls and the capacity of the wall to remove the heat could become a problem if the walls are used as a heat sink, especially in case of large shear rates in the fluid. On the other hand, thermostatting the fluid with a kinetic thermostat requires identification of the streaming and the thermal part of the velocities, furthermore, this approach provides a less realistic model of a 'real' experiment. The difference between both approaches has been analyzed in a number of studies. Some important studies related to thermostatting of confined fluids are summarized here.

The first solid-boundary-driven NEMD study, performed by Liem et al. [37, had as goal to validate the homogeneous shear approach because of the unphysical way that heat is removed in these simulations. The authors found that the difference in stresses between a fluid that is thermostatted via the solid boundaries and a homogeneously sheared fluid was negligible for small strain rates, which is a validation that 
the synthetic thermostatting mechanisms used in homogeneous NEMD simulations are justified in this regime. Sokhan et al. 343] studied the slip at the interface of a fluid in a carbon nanotube (CNT). A thermostatted tube with an unthermostatted fluid was compared to a thermostatted fluid in a rigidly fixed tube. These authors found that, for their system, the difference in velocity slip between both ways of removing the excess heat is less than $20 \%$. In later years, various studies have applied solid boundaries in combination with a thermostatted fluid [57, 336, 344, 345] or a thermostat applied to the walls as well as the fluid [346]. The difference between methods of heat removal might be considered negligibly small for some purposes, this is not true in general. For example, Ma et al. [340] performed simulations in which they cooled the outer part of the channel walls, as well as simulations in which the fluid velocities were rescaled to reach the target temperature. Differences in the phase transition behavior were observed between both approaches. Furthermore, Bernardi et al. [49] recently compared thermostatting methods for confined shear flow and provided a physical interpretation of the difference in slip behavior between fixed and vibrating wall particles. When the wall atoms are mobile, they offer less resistance to the fluid and the fluid is known to have high slip velocities relative to the total streaming velocity typical for a nanochannels.

In Section 2.5 several thermostats were discussed and the difference between a profile-biased thermostat (PBT) and a profile-unbiased thermostat (PUT) was explained. These concepts apply equally to confined fluids, with the additional possibility of thermostatting the boundaries that confine the fluid. Very recently, Yong and Zhang [104], compared homogeneous shear to boundary-driven shear flow. They considered a PBT and a PUT for the homogeneous shear simulations and a PUT for the boundary-driven shear simulations. When the walls are thermostatted, the fluid influences the positions of the walls particles. Due to the generally high pressure in strongly confined liquids, the fluid pushes the walls away from the center of the channel and thus slightly increasing the volume of the channel. Furthermore, fluid particles can penetrate the wall when a fluid particle has a high impact velocity or when the stiffness of the wall potential is low. An additional repulsive potential is sometimes used for fluid-wall interactions to ensure that the fluid does not penetrate the wall [347. In Section 7.4. we apply a Nosé-Hoover thermostat in a profile-unbiased approach, locally to the fluid. The PUT is applied near the walls, where most heat is created due to large velocity gradients. By applying the thermostat only near the walls, the dynamics of the atoms in the center of the channel is purely Newtonian. Furthermore, thermostatting the fluid prevents thermal slip between the fluid and wall, such that the fluid temperature does not deviate much from the target temperature under the influence on a body force. 
Slip between fluid and wall is another important property that influences the flow through a channel [295, 348,354]. Conventional hydrodynamics assumes zero slip in macroscopically large systems. This assumption does not hold true on a molecular level. The observed amount of slip in MD simulations is strongly dependent on the characteristics of the wall, the fluid and various simulations parameters and system specifications. Kobryn and Kovalenko [355] recently studied the structure of 9 inhomogeneous molecular fluids near (100) fcc walls of 3 different materials. They performed equilibrium molecular dynamics (EMD) simulations and used statistical mechanics techniques to calculate the hydrodynamic slip length, as well as the friction coefficient of the fluid-solid interfaces.

Slip lengths are measured by extrapolating the velocity profile linearly, using the slopes of the profile directly next to the walls. This approach can lead to slip lengths that are dependent on how the wall position is defined and confined oscillatory flows definition is not applicable 326]. It is known that the slip velocity varies with the driving field, whereas the slip length is approximately constant in the linear region 356. The influence of the wall properties on the slip velocity for a molecular fluid is shown, for example, in Ref. [357], where the authors compared linear and branched chain molecules confined between weakly and strongly repulsive atomistic walls. The amount of slip can be reduced by decreasing the length scale of the fluid-wall interaction $\sigma_{f w}$ and increasing the energy scale $\epsilon_{f w}$. This allows the fluid atoms to come closer to the walls and increases the effort it takes them to move away from the wall. This not only reduces the slip, but also enhances the formation of fluid layers parallel to the wall. Similar effects can be seen if one fixes the wall atoms in a regular lattice. When the pressure in the fluid is large, the outer fluid atoms are pushed towards the wall, where they look for a position that corresponds to a least amount of potential energy. Some atoms find this position by becoming part of the lattice as was shown in Figure 7.2 .

\subsection{Spatial averaging and macroscopic fields}

In many cases, it is acceptable to only consider the macroscopic scale with a method such as finite elements or finite volumes. However, molecular dynamics simulations are often used to study problems in which the microscopic origin of macroscopic behavior is important. The characteristic scales of microscopic information and macroscopic fields are often a few orders of magnitude apart. When a clear scale separation is true, a macroscopic field could often be calculated from the microscopic information without ambiguity. The transition from microscopic to macroscopic information is more problematic when macroscopic quantities show variations over a length scale 
close to the microscopic length scale. Strongly confined fluids are an example in which bridging the gap from the microscopic information to macroscopic fields (e.g., stress, temperature) is an open problem. While it is known that atoms and molecules are not distributed homogeneously very close to a solid interface, little is known about the resulting spatial variations in the various macroscopic field, since many of these profiles cannot be measured experimentally. Similarly, experimental measurements of transport coefficients assume the fluid to be at a well-defined state, while in practice the state of a fluid is a function of the position in very narrow slit pores.

There are several possible methods to obtain fields as a function of position by sampling microscopic information. The simplest and most common way to do this, is by dividing the volume up into slabs (1D), bins (2D) or bricks (3D) and assigning microscopic information to a single one of these elements. This method is sensitive to the amount of data available and the required spatial resolution of the resulting field. If the elements are very small, it requires a large amount of data to accumulate enough statistics. Large elements, on the other hand, might not capture variations that occur over a small length scale.

The statistics can be improved somewhat by averaging over time (if the system is in a steady state) and the directions in which the fluid can be considered homogeneous. However, even when a reasonably smooth profile is obtained, it is not continuously differentiable. Furthermore, initial information cannot be extracted once it is assigned to a bin or some sort. Finally, the size of the elements is unrelated to the finite size of the particles.

A slight improvement is achieved by assigning information to the actual position of the particle via a Dirac delta function $\delta$. For a quantity $\chi$, the microscopic density of that quantity is given by

$$
\chi(\mathbf{r}, t)=\sum_{i=1}^{N} \chi_{i} \delta\left(\mathbf{r}-\mathbf{r}_{i}(t)\right) .
$$

For example, the microscopic mass density at point $\mathbf{r}$ and time $t$ is calculated via

$$
\rho^{m}(\mathbf{r}, t)=\sum_{i=1}^{N} m_{i} \delta\left(\mathbf{r}-\mathbf{r}_{i}(t)\right),
$$

where $m_{i}$ is the mass of atom $i, \mathbf{r}_{i}$ is its position and $N$ the number of fluid atoms. Other quantities can be defined in a similar fashion [358. This expression preserves the exact position of the information and assigns it to a single position. Yet, numerically the Dirac delta function can only be evaluated as a function with a finite thickness and height, while preserving a surface of 1 under the function. Even with a finite thickness, the function does not represent the spatial distribution of the mass of a 
particle. Neither does the microscopic profile lead to the continuous gradients required to satisfy the balance equations of mass momentum and energy.

A finite number of point-particles in continuous space implies that the mass is zero everywhere, except at the atoms' center of mass. The discontinuities in this (that lead to singular derivatives) can be avoided by averaging over discrete volumes in space, such as binning. However, information is lost in the binning process, i.e., it is impossible to recover the raw data from the bin-averaged values. Furthermore, it requires a large amount of statistics to obtain a smooth microscopic field, without averaging out smallscale physical structures, by using bin averaging. These disadvantages of binning can be avoided by using a more convenient smoothing method.

Lucy [359] and Monaghan 360] independently introduced a method to smoothen the data. The data can be smoothened by replacing the Dirac delta function in Eq. (7.6) by a smoothing function $\phi$. While this technique has been introduced in the field of astrophysics, it proves to be very convenient in analyzing simulations of granular materials [89] and atomic fluids [88, 361]. Spatial smoothing decreases the fluctuations that are associated with the microscopic thermal vibrations of the atoms, furthermore it has the additional advantage of obtaining differentially continuous profiles. However, care must be taken in how to smoothen the data without loosing relevant information. This needs to be addresses on a case-to-case basis. The shape and the width of the kernel determines how the data is diffused over space. Two commonly used kernels are the Lucy function and a Gaussian function.

A Gaussian kernel is given by

$$
\phi_{G}(r)=\frac{1}{\left(\sqrt{2 \pi w^{2}}\right)^{d}} \mathrm{e}^{-\frac{r^{2}}{2 w^{2}}},
$$

where the dimensionality of the smoothing of the data is denoted with $d$, the variance, $w^{2}$, determines the amount of smoothing, while preserving the shape and the area under the curve and $r=|\mathbf{r}|$ is the distance from the center of the kernel. The smoothing kernel has the dimensions of inverse volume, therefore, integrating the kernel over a volume gives a dimensionless quantity. The pre-factor of the Gaussian is normalized the function such that the integral over a $d$-dimensional kernel gives unity.

Lucy's function is given by

$$
\phi_{L}(r)=C_{d}\left(1-6\left(\frac{r}{h}\right)^{2}+8\left(\frac{r}{h}\right)^{3}-3\left(\frac{r}{h}\right)^{4}\right),
$$

where $h$ determines half the width of the kernel and $C_{d}$ is the dimension-dependent constant that ensures that the kernel is normalized. The constants are: $C_{1}=5 /(4 h)$, $C_{2}=5 /\left(\pi h^{2}\right), C_{3}=105 /\left(16 \pi h^{3}\right)$. The larger $h$ is, the more information is smeared out. The limiting case of $h \rightarrow 0$ approached the 'point-particle' case shown in Eq. (7.6). The Lucy kernel is twice continuously differentiable and does not need to be cut off. 


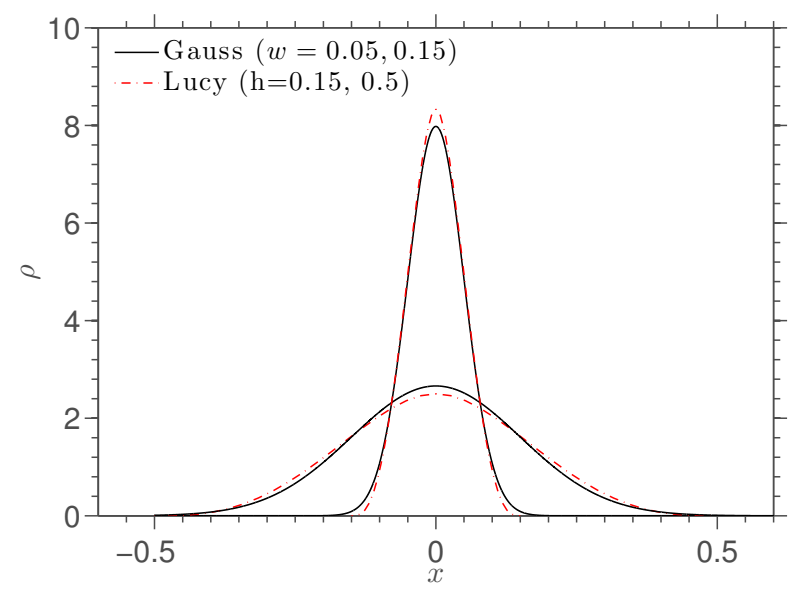

Figure 7.5: Mass density profile for a single atom at position $x=0$, a Gaussian kernel is used with smoothing widths $w=0.05$ and 0.2 and a Lucy kernel with widths $h=0.15$ and 0.5 .

Figure 7.5 shows the density field of a single atom smoothed with a Gaussian kernel with smoothing widths $w=0.05$ and 0.15 and a Lucy kernel with widths $h=0.15$ and 0.5. The shape of both kernels is quite similar and both kernels are normalized such that the integral over the kernel satisfies $\int_{V} \phi(r) d V=1$. However, there are differences between both approaches. The main advantage of a Gaussian is that it is infinitely continuously differentiable. The Lucy weight function has a compact support. That means that the function value vanishes at a certain distance from its center. The Gaussian on the other hand needs to be truncated somewhere and renormalized in order to conserve quantities. The function is no longer smooth at the point of truncation.

Goldhirsch 89] described the requirements of a kernel in detail and stated that it is of minor importance which function is used. The level of smoothing, or smoothing length, on the other hand, can have a large influence on the macroscopic fields. When the obtained macroscopic fields are not strongly dependent on the smoothing length, for a range of values ('plateau'), then the smoothing possibly creates a meaningful macroscopic field. The existence of a plateau and the appropriate amount of smoothing strongly depends on the system. For a detailed discussion, the reader is directed to Goldhirsch [89] and references therein.

The kernel smoothing applied to a configurational stress profile is demonstrated in Figure 7.6, where the one and two-dimensional mass density profiles are shown for a system with 5 particles. The data is smoothed using a Lucy kernel with smoothing 

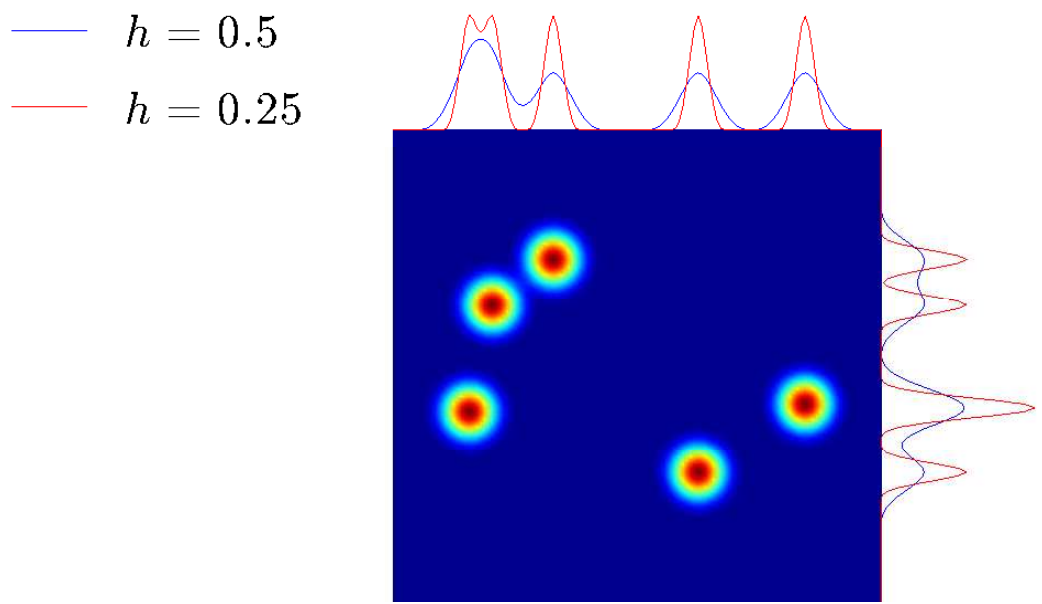

Figure 7.6: One and two-dimensional smooth density profiles for five fixed atoms. The data is smoothed using a Lucy kernel with smoothing widths $h=0.25$ and 0.5.

widths $h=0.25$ and 0.5 .

The density distribution that was shown in Figure 7.1 is created in a similar way as Figure 7.6. The three-dimensional data in Figure 7.1 is averaged over the $z$-direction and over time, such that the information is smoothly distributed across the $x-y$ plane. Similarly, information can be distributed smoothly in all spatial directions with a three-dimensional smoothing function.

\subsubsection{Streaming velocity, strain rate and temperature}

The streaming velocity $\mathbf{u}$ can be calculated from the ratio between momentum and mass density

$$
\mathbf{u}(\mathbf{r})=\frac{\mathbf{J}(\mathbf{r})}{\rho(\mathbf{r})}=\frac{\sum_{i=1}^{N} m_{i} \mathbf{v}_{i} \phi\left(\mathbf{r}-\mathbf{r}_{i}\right)}{\sum_{i=1}^{N} m_{i} \phi\left(\mathbf{r}-\mathbf{r}_{i}\right)},
$$

where $\rho(\mathbf{r})$ is the mass density and $\mathbf{J}(\mathbf{r})$ the momentum density, with $\mathbf{v}_{i}$ the velocity of atom $i$. The velocity gradient $\nabla \mathbf{u}$ can be calculated analytically from the mass and momentum density and their gradients by applying the quotient rule to Eq. 7.10

$$
\nabla \mathbf{u}=\frac{\rho \nabla \mathbf{J}-\mathbf{J} \nabla \rho}{\rho^{2}} .
$$


Evaluating this expression numerically generally results in a strain rate profile that has much larger relative noise than the velocity profile [326].

The kinetic temperature is computed straightforwardly from the fluctuation velocities $\mathbf{v}_{i}^{\prime}$ of the atoms following the expression

$$
T(\mathbf{r})=\frac{2 K^{\prime}(\mathbf{r})}{\operatorname{dn}(\mathbf{r})}=\frac{1}{\operatorname{dn}(\mathbf{r})} \sum_{i=1}^{N} m_{i} \mathbf{v}_{i}^{\prime} \cdot \mathbf{v}_{i}^{\prime} \phi\left(\mathbf{r}-\mathbf{r}_{i}\right)
$$

where $K^{\prime}$ is the kinetic fluctuation energy density, $d$ is the dimension of the system, $\mathbf{v}_{i}^{\prime}=\mathbf{v}_{i}-\mathbf{u}(\mathbf{r})$ is the peculiar velocity of atom $i$, defined as the difference between the laboratory velocity $\mathbf{v}_{i}$ and the streaming velocity $\mathbf{u}$ at the location of the function evaluation $\mathbf{r}$. The kinetic fluctuation temperature is kept constant in the simulations by means of local thermostatting [345, see Section 7.4.1.

\subsubsection{Stress calculation}

Calculating the local stress in strongly confined dense fluids has been a much studied subject [85, 87, 90, 95, 305, 362,364]. Various expressions have been derived, differing mostly in their physical interpretation. The first stress tensor for inhomogeneous fluids was introduced by Irving and Kirkwood [85. In later years, a number of methods have been developed to calculate the local stress tensor in an inhomogeneous fluid [8587, 90, 362, 363. Shen and Atluri [90] derived an atomistic stress tensor by using an approach based on kernel interpolation. This method is easy to implement and results in a continuous stress field. Furthermore, they showed that this method, in contrast to many other widely used methods, satisfies the conservation of linear momentum. Goldhirsch [89] discussed in much detail the advantages and limitations of calculating macroscopic fields from smoothed microscopic data. The method introduced by Schofield and Henderson [87] is used here in combination with a smoothing kernel.

The (compressive) stress tensor for inhomogeneous fluids shown in Section 2.4 (Eq. 2.30) ${ }^{3}$ Replacing the Dirac delta function with a suitable smooth kernel $\phi$ yields

$$
\boldsymbol{\sigma}(\mathbf{r})=\sum_{i=1}^{N}\left(m_{i} \mathbf{v}_{i}^{\prime} \mathbf{v}_{i}^{\prime} \phi\left(\mathbf{r}-\mathbf{r}_{i}\right)+\frac{1}{2} \sum_{j \neq i} \mathbf{r}_{i j} \mathbf{F}_{i j} \int_{0}^{1} d \lambda \phi\left(\mathbf{r}-\mathbf{r}_{i}+\lambda \mathbf{r}_{i j}\right)\right),
$$

where $\mathbf{r}_{i j}=\mathbf{r}_{i}-\mathbf{r}_{j}$.

Figure 7.3.2 shows the configurational part of Eq. 7.13 for two atoms on a distance of $\left|\mathbf{r}_{i j}\right|=1$. The configurational stress is smoothed with a Gaussian kernel with smoothing widths $w=0.05$ and 0.15 .

\footnotetext{
${ }^{3}$ The compressive stress tensor is identical to the pressure tensor, $\boldsymbol{\sigma}=\mathbf{P}$. We use the stress tensor
} 


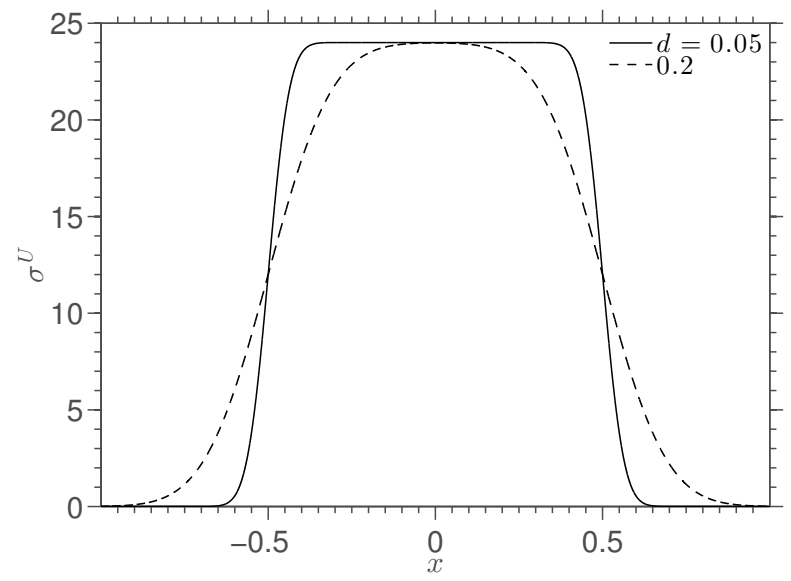

Figure 7.7: Configurational stress profile for two atoms at a fixed distance $\left|\mathbf{r}_{i j}\right|=1$, a Gaussian kernel is used with smoothing widths $d=0.05$ and 0.2 .

The pressure is now also a function of position, such that the definition given in Eq. (2.31) is rewritten as

$$
p(\mathbf{r})=\frac{1}{3} \operatorname{tr}(\boldsymbol{\sigma}(\mathbf{r})) .
$$

It must be noted that Barisik and Beskok 304 concluded recently that it is impossible to define pressure in the near-wall region of strongly confined fluids, due to the anisotropy in the diagonal components of the stress tensor.

Normal stress differences are commonly used as a measure for the deviation from Newtonian behavior of a fluid, as discussed in Section 5.1. For example, colloidal and granular materials exhibit non-Newtonian phenomena such as stress anisotropy, see Alam and Luding [208] and references therein. Structure formation and correlated collisions, for smooth inelastic hard spheres, can lead to non-Newtonian flow with anisotropy in stress, but even an elastic atomic fluid has a small but non-zero anisotropy (normal stress differences) [365]. For example, Sofos et al [298] studied the anisotropy in the transport properties for a confined simple liquid. The authors focussed on the diffusion in the directions parallel and perpendicular to the walls. They observed a lower diffusion in the direction perpendicular to the wall compared to the directions parallel to the wall. They concluded that the transport properties deviate considerably from those of a bulk fluid if the channel width is below a critical value, which is about $8 \sigma-20 \sigma$ for their system.

in this chapter since this is more common in the confined-fluid literature, including the paper that this chapter is based on (Ref. [240]) 
While the normal stresses are relatively easy to measure from experiments, they are not objective under rotation of the coordinate system and therefore not the most suitable quantity to quantify the (objective) anisotropy in stress. Instead of looking at the normal stresses, we define a measure for stress anisotropy in terms of the principal stresses. Objective quantities related to stress are its invariants and the eigenvalues. The latter are related also to their respective eigendirections, which complete the picture. The trace of the stress tensor (Eq. (7.14) ) gives the pressure and is also the first invariant.

One possible definition of the stress anisotropy is the difference between the maximum $\lambda_{1}$ and minimum $\lambda_{3}$ principal deviatoric stress, scaled by twice the pressure $p$

$$
S_{D}(\mathbf{r})=\frac{\lambda_{1}(\mathbf{r})-\lambda_{3}(\mathbf{r})}{2 p(\mathbf{r})} .
$$

An alternative definition for anisotropy, that also involves the intermediate eigenvalue $\lambda_{2}$, is:

$$
S_{D}^{*}(\mathbf{r})=\frac{1}{\sqrt{6} p(\mathbf{r})} \sqrt{\left(\lambda_{1}(\mathbf{r})-\lambda_{2}(\mathbf{r})\right)^{2}+\left(\lambda_{2}(\mathbf{r})-\lambda_{3}(\mathbf{r})\right)^{2}+\left(\lambda_{1}(\mathbf{r})-\lambda_{3}(\mathbf{r})\right)^{2}},
$$

where the term under the square-root is proportional to the second invariant of the deviatoric stress [100, 366]. Both definitions $S_{D}$ and $S_{D}^{*}$ are identical for homogeneous shear flow, when $\lambda_{1}=-\lambda_{3}$ and $\lambda_{2}=0$, as would be the case for a Newtonian fluid.

In hydrodynamic theory of simple liquids, the shear viscosity is simply the constant proportionality factor in the linear constitutive relation between shear stress and strain rate. The Navier-Stokes shear viscosity is given by

$$
\eta=-\frac{\sigma_{x z}}{\dot{\gamma}}
$$

This constitutive relation becomes a very inaccurate approximation for anisotropic, inhomogeneous fluids and the viscosity is, in general, a tensorial, non-constant quantity. In the present study, only a scalar viscosity is considered in the attempt to simplify, while the tensorial nature is taken in to account via other means, see Sections 7.4.2 and 7.4.3 This scalar viscosity approaches a Newtonian viscosity in the bulk region, whereas it is known to be inaccurate where the fluid is strongly inhomogeneous [367.

\subsection{A study of a fluid confined in a nanochannel}

\subsubsection{Model system}

The system is a slit bounded in the $x$-direction by two parallel atomistic walls as shown in Figure 7.8. Periodic boundary conditions are applied in the $y$ - and $z$-direction. The 


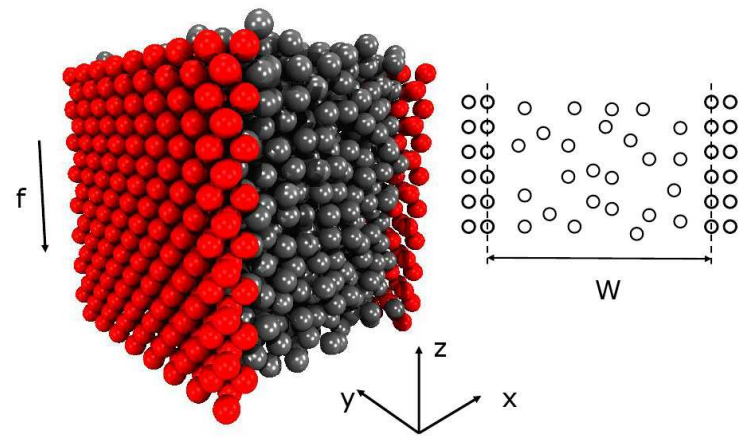

Figure 7.8: left: a snapshot of the system, right: a schematic cross-section indicating the definition of the channel width.

height and the depth of the system are $13.68 \sigma$, with $\sigma$ the length scale of the atoms (i.e., the distance at which the potential energy between a pair of interacting atoms is zero). Either wall is composed of two 001 fcc layers. Each layer is a square lattice, containing 128 atoms fixed at their lattice site, with a spacing of $1.21 \sigma$ between the atoms. The separation distance between the walls is $W=11.1 \sigma$. The width is defined as the distance between the center of the inner wall layers (see Figure 7.8). A flow of liquid argon is simulated in the slit, with $N=1536$ fluid atoms.

We generate a planar Poiseuille flow by applying a constant body force $f$ to the fluid atoms, acting in the negative $z$-direction. Hence, the evolution of the velocity of the atoms is described by Eq. (7.4), with the body force vector given by $\mathbf{F}^{E}=(0,0,-f)$. The magnitude of the body force must be chosen such that the signal-to-noise ratio is large, since otherwise a very large simulation time is required in order to obtain accurate statistics. On the other hand, if the body force is too large, the response of the system becomes very nonlinear and the temperature will vary considerably across the channel [345, 368 371.

The interactions between neutral spherical atoms, such as argon, are well described by a 12-6 Lennard-Jones pair potential 61]

$$
U(r)=4 \epsilon\left[\left(\frac{\sigma}{r}\right)^{12}-\left(\frac{\sigma}{r}\right)^{6}\right],
$$

where $\epsilon$ is the potential well-depth and $r=\left|\mathbf{r}_{i j}\right|=\left|\mathbf{r}_{i}-\mathbf{r}_{j}\right|$ is the absolute distance between the centers of the interacting atoms $i$ and $j$. The potential is truncated at $r=r_{c}=2.5 \sigma$ in order to reduce calculation time. The potential is shifted down by the value $U\left(r_{c}\right)$ in order to avoid a discontinuity at the cut-off distance. The force between atoms is

$$
\mathbf{F}_{i j}=-\frac{d U}{d r_{i j}} \frac{\mathbf{r}_{i j}}{r}
$$


where $\mathbf{F}_{i j}$ is the force acting on atom $i$ due to atom $j$. Interactions between wall and fluid atoms are calculated in the same way as interactions between a pair of fluid atoms.

The body force that acts on the atoms generates thermal energy leading to a temperature rise in the system. To control the temperature, the generated heat needs to be removed from the system. This is done via the Nosé-Hoover thermostat, which couples the atoms to a thermal reservoir 121. In nature, heat is transported to the thermostatted walls and the exchange of momentum and heat between the wall and the fluid takes place. We could try to mimic nature by allowing wall atoms to vibrate around their lattice sites and controlling the average temperature of the walls. However, since thermal walls would lead to a decrease in the near-wall inhomogeneity in which we are interested, we choose to fix the wall atoms and thermostat the fluid locally next to the walls in order to obtain a constant temperature profile [49, 345] and avoid the thermal slip [11, 348] that would occur when the walls are thermostatted instead of the fluid. Since shear generates most heat in the vicinity of the walls, the fluid is locally thermostatted in this region, but not in the center (bulk) region. On both sides of the channel, three thermostats are located next to each other, each of width 1 . The first thermostat, seen from the wall, begins on a distance of 0.15 from the center of the inner wall layer. Thus, a region of approximately 4.8 wide, in the center of the channel, is not thermostatted. This approach maintains a rather constant temperature profile in the fluid, as long as $f$ is not too large, while a global thermostat does not always succeed [344, 345] due to the strong variation in strain-rate across the channel.

For the system studied here, a Gaussian smoothing kernel is used. The obtained macroscopic field is then averaged over the $y$ and $z$-directions, as well as over time. The smoothing has to be small enough such that the width of the Gaussian is narrow compared to the length scales of the spatial inhomogeneities observed in strongly confined fluids, but large enough to eliminate the thermal fluctuations from the macroscopic fields. A value of $w=0.1$, as will be used below, has shown to satisfy these conditions and result in fields which do not strongly depend on the value chosen for $w$. The kernel is cut off at a distance of $3.0 \mathrm{w}$ from the center and renormalized such that the volume under the kernel is unity and thus the amount of information is preserved. In addition to spatial smoothing, the steady-state simulation data are averaged over discrete snapshots in order to increase the statistics.

The calculation of the streaming velocity and strain rate profiles were presented in Section 7.3.1. Alternatively, the streaming velocity and strain rate can be calculated from the displacement field. Averaging the strain rate over a time interval $\Delta t$ offers additional spatial and temporal smoothing compared to the velocity gradient and 
hence reduces noise. Therefore, we compute the linear displacement field over a time interval $\Delta t$, as defined in Ref. [372],

$$
\mathbf{U}^{\operatorname{lin}}(\mathbf{r}, t)=\frac{1}{\rho(\mathbf{r}, t)} \sum_{i=1}^{N} m_{i} \mathbf{U}_{i}(t) \phi\left(\mathbf{r}-\mathbf{r}_{i}(t)\right),
$$

with $\mathbf{U}_{i}(t)=\mathbf{r}_{i}(t)-\mathbf{r}_{i}(t-\Delta t)$ the displacement of atom $i$ during time interval $\Delta t$. The linear strain can then be computed from the displacement gradient

$$
\epsilon_{\alpha \beta}^{\operatorname{lin}}(\mathbf{r}, t)=\frac{1}{2}\left[\frac{\partial U_{\alpha}^{\operatorname{lin}}(\mathbf{r}, t)}{\partial r_{\beta}}+\frac{\partial U_{\beta}^{\operatorname{lin}}(\mathbf{r}, t)}{\partial r_{\alpha}}\right] .
$$

In Fig. 7.10 (Section 7.4.3), we compare the streaming velocity with the displacement rate $\mathbf{U}^{\operatorname{lin}}(\mathbf{r}, t) \Delta t^{-1}$, and the velocity gradient with the strain rate $\epsilon_{\alpha \beta}^{\text {lin }} \Delta t^{-1}$, where $\Delta t$ is the time interval between snapshots. As expected, the displacement and strain rates over a time interval $\Delta t$ are smoother than the velocity field and its gradient, respectively. The viscosity, calculated with the displacement method is given by

$$
\eta=-\frac{\Delta t \sigma_{x z}}{\epsilon_{x z}^{l i n}}
$$

\subsubsection{Constitutive model with anisotropic stress}

The relations between macroscopic quantities (such as those derived in Section 7.3) can be described in terms of a constitutive model. If only sufficiently small body forces are considered, the system can be treated as longitudinally homogeneous and the fields can be averaged over the directions parallel to the walls (i.e., the $y$ - and $z$-direction) 86. Since the fields vary only in $x$-direction and we are interested in a constitutive model for an anisotropic, inhomogeneous fluid, only the direction perpendicular to the walls is considered here as spatial variable.

Following the same approach as in Chapter5. One can decompose the stress into an isotropic (pressure) and a deviatoric part

$$
\boldsymbol{\sigma}=p \mathbf{1}+\boldsymbol{\sigma}^{D}
$$

where $\mathbf{1}$ is the unit tensor, and $\boldsymbol{\sigma}^{D}$ is the (trace-free) deviatoric stress. For a Newtonian fluid, the second term is the viscous stress component

$$
\boldsymbol{\sigma}_{N}^{D}=-\eta \boldsymbol{S}=-\eta\left(\nabla \boldsymbol{u}+(\nabla \boldsymbol{u})^{\mathrm{T}}\right)
$$

where $\eta$ is the shear viscosity, and $\boldsymbol{S}$ the strain rate tensor (i.e., the symmetrized velocity gradient $\nabla \boldsymbol{u}$, where the transposed is indicated by a superscript T). Note that the pressure and the shear viscosity are constant across the system in a homogeneous 
Newtonian fluid at constant temperature. A positive pressure $p$ indicates that the system is dominated by repulsive forces, according to our sign convention.

In a planar Poiseuille geometry where $u_{z}$ is the only non-zero component of the streaming velocity, the symmetric strain rate tensor is given by

$$
\boldsymbol{S}=\left(\begin{array}{ccc}
0 & 0 & \dot{\gamma} \\
0 & 0 & 0 \\
\dot{\gamma} & 0 & 0
\end{array}\right)
$$

The strain rate tensor $\boldsymbol{S}$ can also be expressed in terms of its eigenvalues $\pm \dot{\gamma}$ and eigen-orientation $\alpha$ (with $\alpha= \pm \frac{\pi}{4}$ ), representing the magnitude and the orientation of the tensile $(+)$ and compressive $(-)$ direction of the strain rate, respectively. As convention, we define 'the orientation' of the tensor as the angle $\alpha$ between the horizontal and the largest (positive) eigenvalue. Consequently

$$
\boldsymbol{S}=\dot{\gamma} \boldsymbol{D}(\alpha)=\dot{\gamma} \boldsymbol{R}(\alpha) \cdot \mathbf{1}_{D} \cdot \boldsymbol{R}^{\mathrm{T}}(\alpha)=\dot{\gamma} \boldsymbol{R}(\alpha) \cdot\left(\begin{array}{ccc}
1 & 0 & 0 \\
0 & 0 & 0 \\
0 & 0 & -1
\end{array}\right) \cdot \boldsymbol{R}^{\mathrm{T}}(\alpha),
$$

which defines a unit-deviator $\boldsymbol{D}(\alpha)$, where a special case is $\mathbf{1}_{D} \equiv \boldsymbol{D}(0)$, with the eigenvectors rotated (counter-clockwise) about an angle $\alpha$ around the $y$-axis, i.e., inside the $x$-z-plane, with the rotation matrix

$$
\boldsymbol{R}(\alpha)=\left(\begin{array}{ccc}
\cos \alpha & 0 & -\sin \alpha \\
0 & 1 & 0 \\
\sin \alpha & 0 & \cos \alpha
\end{array}\right)
$$

that rotates a vector about an angle $\alpha$ in counter-clock-wise direction around the $y$-axis (with the $y$-axis pointing away from the observer) when acting on it, e.g., $\boldsymbol{R}(\alpha)(1,0,0)^{\mathrm{T}}=(\cos \alpha, 0, \sin \alpha)^{\mathrm{T}}$.

Substituting $\alpha=\pi / 4$ in expression 7.24 yields

$$
\boldsymbol{S}=\dot{\gamma} \boldsymbol{D}(\pi / 4)=\dot{\gamma}\left(\begin{array}{ccc}
0 & 0 & 1 \\
0 & 0 & 0 \\
1 & 0 & 0
\end{array}\right),
$$

which defines the shear unit-deviator $\boldsymbol{D}(\pi / 4)$, with the eigenvectors rotated by an angle of $\alpha=\pi / 4$ around the $y$-axis. Note that the form of the velocity gradient in our system is thus

$$
\boldsymbol{S}=s \boldsymbol{D}\left(\phi_{S}= \pm \pi / 4\right),
$$

throughout the system and the position-dependence only enters in the shear rate, $s=\sqrt{\frac{1}{2} \mathbf{S}: \mathbf{S}}=|\dot{\gamma}(x)| \geq 0$. The sign of the strain-rate orientation, in the planar Poiseuille geometry, corresponds to the left $(-)$ or the right $(+)$ side of the symmetry axis and is contained in $\phi_{S}$, but not in the (positive) shear-rate. 


\section{Non-Newtonian flow for simple shear}

A similar expression can be formulated for a non-Newtonian fluid stress, as studied in the present work. Ideally, for a channel geometry, the constitutive model could be formulated with as little as four variables; one stress (pressure) for the isotropic part, two (eigenvalues of the deviatoric stress) for the anisotropic part and the orientation $\phi_{\sigma}$ of the stress-deviator. Note that in practice, for more general flow situations, additional parameters, e.g. orientations, might be necessary. The constitutive relation then takes the form

$$
\boldsymbol{\sigma}^{D}=\sigma^{D} \boldsymbol{D}\left(\phi_{\sigma}\right)=\eta s \boldsymbol{D}\left(\phi_{\sigma}\right)=\eta s\left[R(\Delta \phi) \cdot \boldsymbol{D}\left(\phi_{S}\right) \cdot R^{T}(\Delta \phi)\right],
$$

with the difference in orientation $\Delta \phi \equiv \phi_{S}-\phi_{\sigma}$ between stress and strain rate tensors and the magnitude of the deviatoric stress tensor $\sigma_{D}=\sqrt{\frac{1}{2} \boldsymbol{\sigma}^{D}: \boldsymbol{\sigma}^{D}}=p S_{D}^{*}$. Even though non-linear due to the rotation operation, the model is objective by construction, since only orientation-differences show up and all quantities with physical units are positive, which allows to define the objective viscosity

$$
\eta_{D} \equiv \frac{\sigma^{D}}{s} \cos (2 \Delta \phi)=\frac{p S_{D}^{*}}{s} \cos (2 \Delta \phi)
$$

as displayed in Section.7.4.3 (Figure 7.16). This expression still relies on the assumption that a stress is locally related to the strain-rate. This measure of viscosity is equal to the Hounkonnou viscosity in Eq. 5.50, and more general than Eq. (7.17). Note that Eq. 7.29) accounts for possible non-collinearity between the stress and strain-rate tensor, which is true in our system (see below). We present an advanced, general model for the deviatoric stress in the next subsection.

\section{A general non-Newtonian flow model for simple shear}

For a non-Newtonian fluid the decomposition of stress in its isotropic and deviatoric parts in Eq. 7.21 contains the pressure $p$, which is now a function of the $x$-position. The second part is the deviatoric stress, which is not simply proportional to the strain rate tensor times a constant scalar viscosity, but contains the rotation of the eigensystem about an angle $\Delta \phi$. For decomposition, an alternative approach needs to be invoked: First, the (deviatoric) stress tensor is rotated by $\alpha=-\phi_{\sigma}$ around the $y$-axis to obtain its diagonal form

$$
\mathbf{R}^{\mathrm{T}}\left(\phi_{\sigma}\right) \cdot \boldsymbol{\sigma}^{D} \cdot \mathbf{R}\left(\phi_{\sigma}\right)=\left(\begin{array}{ccc}
\lambda_{1} & 0 & 0 \\
0 & \lambda_{2} & 0 \\
0 & 0 & \lambda_{3}
\end{array}\right) .
$$

The principal deviatoric stresses $\lambda_{i}$ are the eigenvalues of the deviatoric stress tensor, sorted as $\lambda_{1} \geq \lambda_{2} \geq \lambda_{3}$, the principal orientation follows from the corresponding 
eigenvectors $4^{4}$ Since the trace of the (principal) deviatoric stress tensor is zero, it can be expressed in terms of two principal stresses by substituting $\lambda_{3}=-\left(\lambda_{1}+\lambda_{2}\right)$, consistent with the steps taken in Section 5.3. Splitting the right-hand side of Eq. 7.30 into two tensors and rotating them back to the Cartesian system gives the deviatoric stress

$$
\boldsymbol{\sigma}^{D}=\mathbf{R}\left(\phi_{\sigma}\right) \cdot\left(\lambda_{1}\left(\begin{array}{ccc}
1 & 0 & 0 \\
0 & 0 & 0 \\
0 & 0 & -1
\end{array}\right)+\lambda_{2}\left(\begin{array}{ccc}
0 & 0 & 0 \\
0 & 1 & 0 \\
0 & 0 & -1
\end{array}\right)\right) \cdot \mathbf{R}^{\mathrm{T}}\left(\phi_{\sigma}\right)
$$

For the special case of a Newtonian fluid, one has $\lambda_{1}=\eta|\dot{\gamma}| / 2, \lambda_{2}=0$ and $\phi_{\sigma}=\mp \pi / 4$ for the left and right half of the channel, respectively. In this case, Eq. 7.31 reduces to Eq. 7.22). For a non-Newtonian fluid, however, the pressure, the orientation angle $\phi_{\sigma}$ and the two factors $\lambda_{1}$ and $\lambda_{2}$ of the deviatoric stress can depend explicitly on the position, and e.g., on density or temperature, and on the other variables (and themselves) too. This approach deviates from the decomposition into an in-plane and an out-of-plane tensor, used in Chapter 5. Alternatively, such a decomposition can be given, based on Eq. 7.30 , as

$\boldsymbol{\sigma}^{D}=\left(\lambda_{1}+\frac{\lambda_{2}}{2}\right) \mathbf{R}\left(\phi_{\sigma}\right) \cdot\left(\begin{array}{ccc}1 & 0 & 0 \\ 0 & 0 & 0 \\ 0 & 0 & -1\end{array}\right) \cdot \mathbf{R}^{\mathrm{T}}\left(\phi_{\sigma}\right)+\frac{\sqrt{3} \lambda_{2}}{2}\left(\begin{array}{ccc}-1 / \sqrt{3} & 0 & 0 \\ 0 & 2 / \sqrt{3} & 0 \\ 0 & 0 & -1 / \sqrt{3}\end{array}\right)$.

The tensor on the right is now invariant to rotations in the plane of flow and both tensors have a scalar magnitude of 1 .

Quantifying the deviatoric stress eigenvalues in terms of other quantities is much more difficult for the confined fluid system than it was for the unconfined fluid in Chapter 5 Considering the ratio of $\xi_{\sigma} \equiv \lambda_{2} / \lambda_{1}$ allows to classify the deviatoric stress tensor uniquely according to its 'shape', i.e., values of $\xi_{\sigma}=1,1 / 2,0$, and $-1 / 2$ correspond to the special cases of (i) axial tension, (ii) mixed, (iii) simple shear, and (iv) axial compression, respectively. The ratio $\xi_{\sigma}$ is strongly oscillating across the channel between values somewhat larger than $+1 / 2$ and $-1 / 2$. This behavior is shown below in Figure $7.26(\mathrm{~b})$,

\section{Non-Newtonian Flow model - special cases}

The magnitude of $\lambda_{2}$ and the difference in orientation $\Delta \phi=\phi_{S}-\phi_{\sigma}$ are both quantifying the deviation from ideal Newtonian flow behavior. The stress-anisotropy definitions from Eqs. 7.15 and 7.16) thus translate to $S_{D}=\lambda_{1}\left(1+\xi_{\sigma} / 2\right) / p=\left(\lambda_{1}+\lambda_{2} / 2\right) / p$ and $S_{D}^{*}=\lambda_{1} \sqrt{1+\xi_{\sigma}+\xi_{\sigma}^{2}} / p=\sqrt{\lambda_{1}^{2}+\lambda_{1} \lambda_{2}+\lambda_{2}^{2}} / p$ - identical to first order in the limit case $\lambda_{2} \ll \lambda_{1}$.

\footnotetext{
${ }^{4}$ The rotation matrix contains the normalized eigenvectors as columns.
} 
Case 1: $\lambda_{2}=0, \Delta \phi \neq 0$

Thus, even for the second eigenvalue vanishing, i.e., $\lambda_{2}=0$, the flow behavior can be classified as non-Newtonian if $\Delta \phi \neq 0$. More specific, the special case $\lambda_{2}=0$, for arbitrary non-collinear stress-strain relations 5 is equivalent to

$$
\begin{aligned}
\boldsymbol{\sigma}^{D} & =\lambda_{1}\left(\begin{array}{ccc}
\cos ^{2}\left(\phi_{\sigma}\right)-\sin ^{2}\left(\phi_{\sigma}\right) & 0 & 2 \cos \left(\phi_{\sigma}\right) \sin \left(\phi_{\sigma}\right) \\
0 & 0 & 0 \\
2 \cos \left(\phi_{\sigma}\right) \sin \left(\phi_{\sigma}\right) & 0 & -\cos ^{2}\left(\phi_{\sigma}\right)+\sin ^{2}\left(\phi_{\sigma}\right)
\end{array}\right) \\
& =\lambda_{1}\left(\begin{array}{ccc}
\cos \left(2 \phi_{\sigma}\right) & 0 & \sin \left(2 \phi_{\sigma}\right) \\
0 & 0 & 0 \\
\sin \left(2 \phi_{\sigma}\right) & 0 & -\cos \left(2 \phi_{\sigma}\right)
\end{array}\right),
\end{aligned}
$$

which leads to $\sigma_{x y}=\lambda_{1} \sin \left(2 \phi_{\sigma}\right)$ and the normal stress differences $\mathcal{N}_{1}=\sigma_{x x}-\sigma_{z z}=$ $2 \lambda_{1} \cos \left(2 \phi_{\sigma}\right)$ and $\mathcal{N}_{2}=\sigma_{x x}-\sigma_{y y}=\mathcal{N}_{1} / 2$.

Case 2: $\Delta \phi=0, \lambda_{2} \neq 0$

In the special (collinear) case $\phi_{\sigma}= \pm \pi / 4$, the deviatoric stress is

$$
\boldsymbol{\sigma}^{D}=\left(\begin{array}{ccc}
-\lambda_{2} / 2 & 0 & \pm\left(\lambda_{1}+\lambda_{2} / 2\right) \\
0 & \lambda_{2} & 0 \\
\pm\left(\lambda_{1}+\lambda_{2} / 2\right) & 0 & -\lambda_{2} / 2
\end{array}\right)
$$

which leads to $\sigma_{x y}= \pm\left(\lambda_{1}+\lambda_{2} / 2\right)= \pm p S_{D}$, and the normal stress differences $\mathcal{N}_{1}=$ $\sigma_{x x}-\sigma_{z z}=0$ and $\mathcal{N}_{2}=\sigma_{x x}-\sigma_{y y}=-3 \lambda_{2} / 2$.

A collinear stress-strain relation with first normal stress difference vanishing is thus equivalent to our model for $2 \mathcal{N}_{2} / 3=-\lambda_{2} \neq 0$. In this case, to be consistent with Eq. (7.28), the (positive) deviatoric stress magnitude above, can be defined as $\lambda_{1}=\sigma_{D}=p S_{D}^{*}=\sqrt{J_{2}\left(\boldsymbol{\sigma}^{D}\right)}=\sqrt{1 / 3} \sigma_{\text {von Mises, }}$, i.e., the square-root of the second invariant of the deviatoric stress tensor, and proportional to the well-known von Mises planar stress.

Note that the general non-Newtonian fluid will involve not only a rotation about the $y$-axis, but also around a second axis in the $x$-z-plane, however, we disregard this possibility here, because of the symmetry of the channel flow geometry.

\footnotetext{
${ }^{5}$ i.e., Relations in which the preferred orientations of stress and strain are not assumed to be identical.
} 
Case 3: Planar shear: $\sigma_{x y}^{D} \approx \sigma_{y z}^{D}<<\sigma_{x z}^{D}$

Just assuming planar shear, i.e., the second eigenvector in y-direction, and applying the transformation matrix $\mathbf{R}\left(\phi_{\sigma}\right)$ from Eq. 7.31), leads to the deviatoric stress

$$
\boldsymbol{\sigma}^{D}=\left(\begin{array}{ccc}
\lambda_{1} \cos \left(2 \phi_{\sigma}\right)-\lambda_{2} \sin ^{2}\left(\phi_{\sigma}\right) & 0 & \left(\lambda_{1}+\lambda_{2} / 2\right) \sin \left(2 \phi_{\sigma}\right) \\
0 & \lambda_{2} & 0 \\
\left(\lambda_{1}+\lambda_{2} / 2\right) \sin \left(2 \phi_{\sigma}\right) & 0 & -\lambda_{1} \cos \left(2 \phi_{\sigma}\right)-\lambda_{2} \cos ^{2}\left(\phi_{\sigma}\right)
\end{array}\right),
$$

which leads to $\sigma_{x y}=\left(\lambda_{1}+\lambda_{2} / 2\right) \sin \left(2 \phi_{\sigma}\right)=p S_{D} \sin \left(2 \phi_{\sigma}\right)$, and the normal stress differences $\mathcal{N}_{1}=\sigma_{x x}-\sigma_{z z}=2\left(\lambda_{1}+\lambda_{2} / 2\right) \cos \left(2 \phi_{\sigma}\right)=2 \sigma_{x y} \cot \left(2 \phi_{\sigma}\right)$ and $\mathcal{N}_{2}=\sigma_{x x}-$ $\sigma_{y y}=\lambda_{1} \cos \left(2 \phi_{\sigma}\right)-\lambda_{2}\left(1+\sin \left(2 \phi_{\sigma}\right)\right)=\mathcal{N}_{1} / 2-3 \lambda_{2} / 2$. The relation between shear stress, eigenvalues, and orientation angle is perfectly fulfilled in systems in which the principal orientation required only a rotation around the angle perpendicular to the plane of flow. This includes our system. The term $\sin \left(2 \phi_{\sigma}\right)$ is a factor with unitbaseline for $\phi_{\sigma} \approx \pi / 4$ and drops in-between, which compensates the deviations from the linear trend. The IMC equation defined in Eq. 7.38 below, thus provides a relation between density and the three unknowns $\lambda_{1}, \lambda_{2}$, and $\phi_{\sigma}$.

\subsubsection{Results}

In this section, we present various macroscopic fields, among which scalar variables such as: density, temperature and pressure as well as vector fields like streaming velocity and tensorial fields like velocity gradient and stress across the channel. Viscosity as a combined quantity is also discussed. A study of the influence of different temperatures and body forces on the fluid properties are presented in Subsections 7.4 .3 and 7.4.3 respectively. The dependence of density and (to a lesser extend) velocity profiles on body forces and temperature have been well-documented in a number of studies [300, 307, 338. Therefore, we focus on the influence on stress fields and we discuss the aforementioned quantities in less detail, unless our observations deviate from earlier work.

The presented results correspond, unless stated otherwise, to a channel of width $W=11.1$, an average fluid density $\rho=0.8$, body force $f=0.1$ acting in negative $z$-direction and a temperature $T=1.0$. The equations of motion are integrated using a velocity Verlet algorithm with a time step $d t=0.001$. After equilibration, the steady-state simulation results are averaged by means of 5000 snapshots over a period of time of 5000 (i.e., $\Delta t=1$ ). $M=134$ data points are used across the channel, so that the points are separated by $\Delta x=W / M \approx 0.08$. The standard smoothing length is $w=0.1$. For the fits of several quantities in the bulk, the region within a distance of 3.5 of either wall is disregarded as the inhomogeneity is too strong. As mentioned in Section 7.4.1, all quantities are reported in reduced Lennard-Jones units. 


\section{Reference system}

Figure 7.9 shows the density profile, where the oscillations indicate the existence of distinct fluid layers. For a confinement of about $W \leq 11.1$, in combination with the present temperature and average density, this 'layering' occurs across the whole channel, forming a discontinuously structured/layered liquid medium. While the oscillations are present across the whole channel, their magnitude increases towards the walls. In the center, the time- and space-averaged density profile still shows a clear structure, whereas no clear layers are observed in a snapshot of the fluid (not shown). The part of the channel where the fluid behaves (almost) as a bulk fluid, is indicated by the two vertical lines in Figure 7.9. The density profile can be fitted with the product of an oscillating and an exponential function

$$
\rho^{f i t}(x)=\rho_{0}+\alpha \cos \left( \pm \frac{2 \pi}{L}\left(x+x_{w}\right)\right) \exp \left(\frac{\mp\left(x+x_{w}\right)}{x_{0}}\right),
$$

The \pm and $\mp$ signs correspond to a fit near the left and the right wall respectively. Physical properties, such as the bulk density $\rho_{0}$ and the period ( wavelength) $L$ of the density oscillations, can be identified. The exponential decay away from the wall is quantified by $x_{0}$ and the amplitude of the density oscillations (at the wall) are fitted with $\alpha$. It must be noted that, unlike the period of oscillation and the bulk volume fraction, the amplitude and decay of the oscillatory peaks, are strongly dependent on the coarse graining of the data (in this work, a Gaussian function is used to coarsegrain the information). The parameters that fit the data shown in Figure 7.9 near the left wall, are as follows: $\rho_{0}=0.78, \alpha=0.89, x_{w}=-5.61, L=0.93$ and $x_{0}=0.95$. The oscillations in density against the $x$-position for different channel widths was studied in more detail in Hartkamp and Luding [373. Their main result was the observation of well-defined oscillations of wavelength 0.93 , with an exponential decay towards the center of the channel, where the wall effects from left and right can be superposed 373. The exponential decay of the magnitude of the density oscillations away from the walls was also observed in Ref. 374.

As the channel width increases, the layering near the wall remains and loses its dependence on the channel width. Furthermore, the density in the center converges to a bulk density, as the effect of the walls in this region decreases. The magnitude and the extent of the inhomogeneity in density depends, in addition to channel width, on the average fluid density, as well as on the interaction parameters between fluid atoms and between fluid and wall. This parameter dependence is not studied here.

Figure 7.10 shows the streaming velocity in $z$-direction and the derivative of the streaming velocity with respect to $x$. The streaming velocity profile from Eq. 7.10 is approximately quadratic in the bulk (i.e., between the vertical lines in Figure 7.10) and deviates from quadratic near the walls. Similar to density, the velocity profile shows 


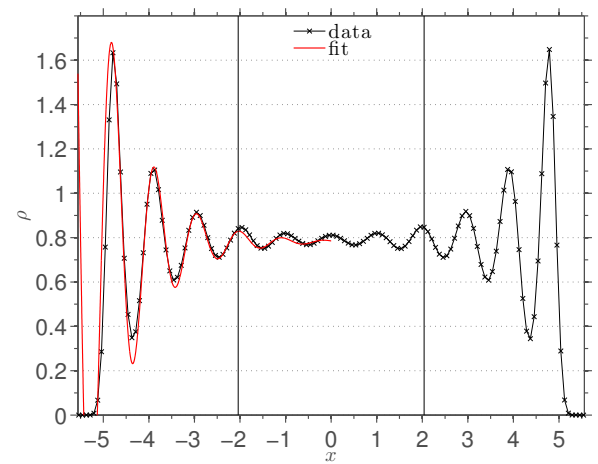

(a)

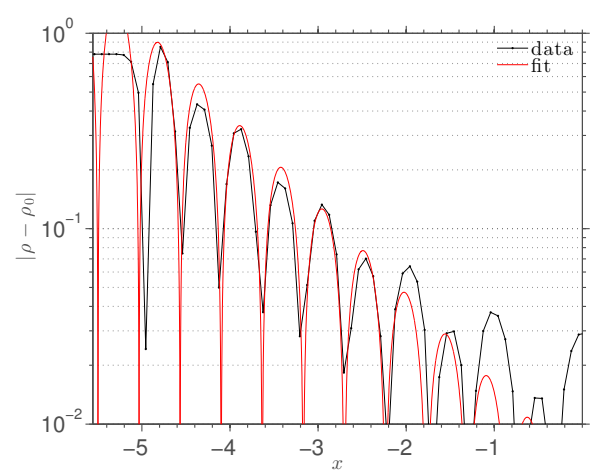

(b)

Figure 7.9: Density from our reference simulation, across a channel of width $W=11.1$ for a body force of $f=0.1$. The density profile is fitted with the function given in Eq. (7.37). The fluid has an average density of $\rho=0.8$ and a temperature $T=1.0$. All quantities are reduced with the Lennard-Jones parameters. The data is averaged over 5000 snapshots over a period of time of 5000 (i.e., $\Delta t=1$ ). The profile shows $M=134$ data points with distance of $\Delta x \approx 0.08$. The range of the $x$-axis is taken to be bound by the centers of wall particles closest to the fluid. The part of the channel between the vertical lines at $x= \pm 2.06$ is where the fluid behaves approximately as a bulk fluid. 


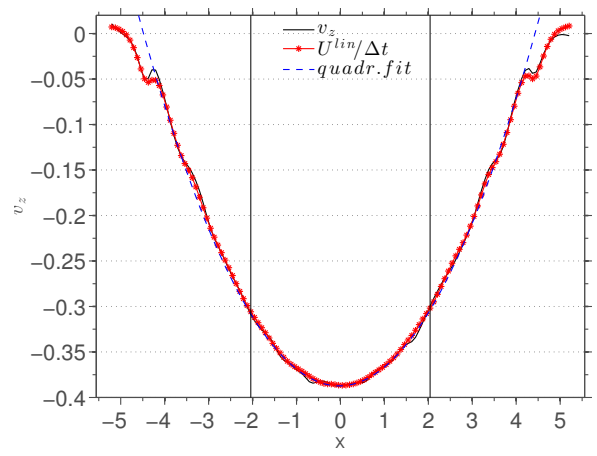

(a)

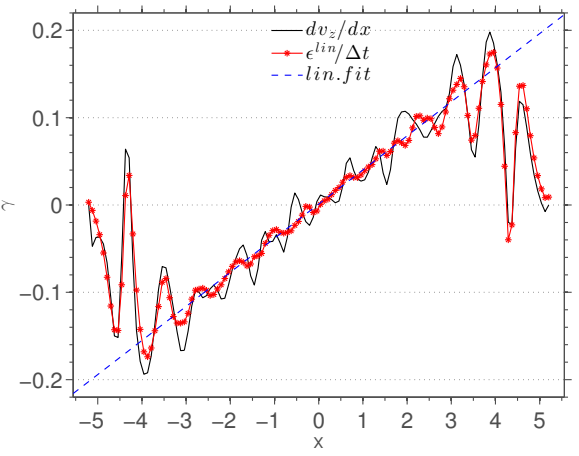

(b)

Figure 7.10: Streaming velocity (a) and strain rate (b) across the channel. The averaged data, displacement averaging method and fits of the averaged data are shown. The simulation and averaging parameters are given in Figure 7.9 . The quadratic fit of the velocity profile is made in the bulk region, that lies between the vertical lines at $x= \pm 2.06$. Differentiating the quadratic fit of the displacement velocity profile with respect to $x$ gives a slope of $\dot{\gamma} / x=0.0401$ for the strain rate profile, consistent with the fit.

variations/oscillations next to the wall, which quickly disappear away from the wall. The oscillations lead to sign changes of the strain rate profile, locally near the walls. This phenomenon is known to occur in strongly confined dense fluids as a consequence of the layering of the atoms [79]. The atoms in the layers (with higher density) move with similar velocity, while slip occurs between them (at low density). Note that the formation of layers is enhanced by the fixed regular lattice walls. This enables us to study a clear breakdown of the continuum behavior in a channel that is wider than in some other studies [375. A quadratic streaming velocity would result in a linear strain rate profile. The averaged profiles in Figure $7.10(\mathrm{~b})$ are approximately linear in the bulk region, oscillate through the layers and drop to zero at the walls (a zero strain rate corresponds to a locally flat streaming velocity profile). When atoms are so close to a wall that they penetrate the lattice, then they do not have the freedom to move in a direction parallel to the wall. Hence, at this $x$-location, the streaming velocity and its gradient approach zero.

Figure 7.11 shows the temperature profile across the channel. It is slightly higher than the target value of $T=1$. Towards the center of the channel, where the fluid is not thermostatted, the average temperature increases up to $T \approx 1.015$ plus or minus fluctuations, that are small compared to the average value. Furthermore, the profile 


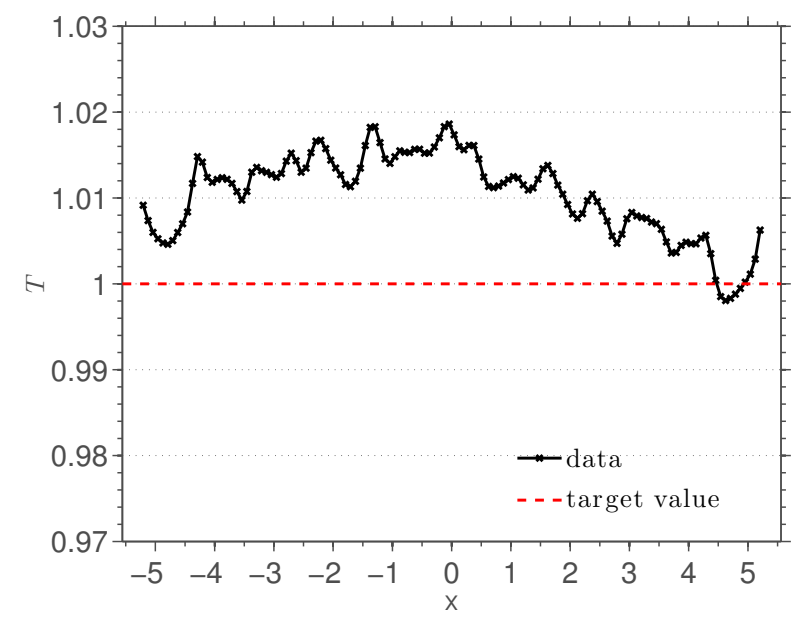

Figure 7.11: Temperature across the channel. The dashed red line indicates the target value.

shows a slight asymmetry due to statistical uncertainty. The fact that the temperature profile is uniform (within $2 \%$ ) across the channel indicates that the local thermostats are sufficient to maintain a constant temperature in the whole domain. In contrast, thermostatting the fluid with a global thermostat has shown to result in a less uniform temperature profile 345. The thermostatting method assumed a constant streaming velocity profile across the thermostatting slabs. In order to verify that the consequences of this assumption are small, a simulation with 12 individually thermostatted layers of width $W=0.5$ (instead of $W=1$ ) is run. No significant difference was noted between the temperature profile from both simulations. The average temperature of the fluid was less than one per cent different for both simulations, though the kinetic and configurational stress are slightly more different $(<1 \%$ and $<3 \%$, respectively).

Figure 7.12 shows the normal stresses and the pressure across the channel. Note that the stresses in a strongly confined fluid are very high; a reduced unit stress $\sigma_{i i}=1$ corresponds to a stress of $\sigma_{i i}^{*}=42 \mathrm{MPa}$ for argon. The fact that the normal stresses are not identical indicates that the stress is anisotropic in general, but here it is isotropic in the $y z$-plane. The (continuum) conservation equation of linear $x$-momentum requires that $d \sigma_{x x} / d x=0$ in steady-state, which is approximately satisfied by the constant profile for $\sigma_{x x}$ if the system is in mechanical equilibrium. The average value of $\sigma_{x x}$ agrees, within one percent, with the nominal stress (i.e., the time-averaged force on the walls divided by the area of the walls), which is denoted with ' $\times$ '. The derivatives of the other normal stresses with respect to $x$ are not restricted by the conservation 


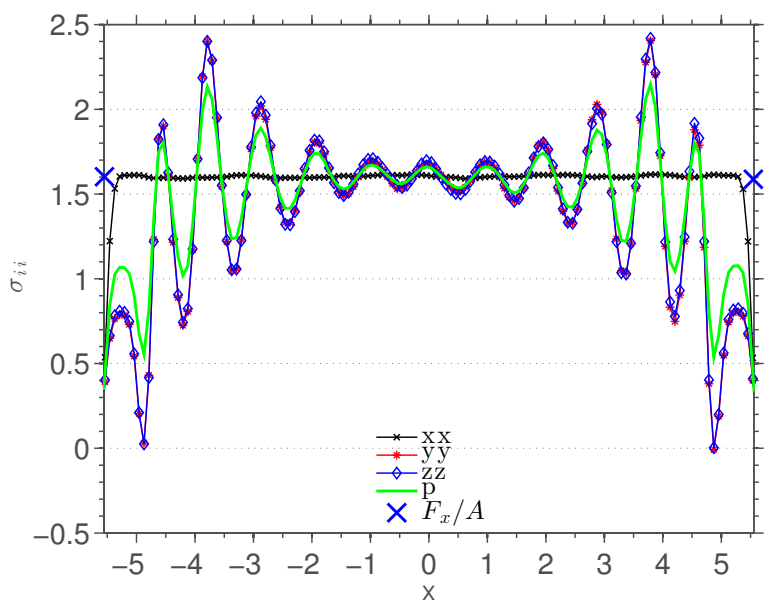

Figure 7.12: Normal stresses components (where the labels $x x, y y$ and $z z$ refer to the ii component of the stress tensor) and pressure $p$ across the channel. The nominal stress on the walls is shown on the left and right side as crosses $\times$.

equations. The profiles of $\sigma_{y y}$ and $\sigma_{z z}$ oscillate near the walls and approach the value of $\sigma_{x x}$ in the center of the channel. Since the pressure is the average of the normal stresses, the pressure profile shows a similar oscillatory behavior as $\sigma_{y y}$ and $\sigma_{z z}$, with smaller oscillations. The peaks and troughs in pressure roughly (but not exactly) correspond to a high and low local density, respectively.

Figure 7.13 shows the kinetic and configurational parts of normal stresses $\sigma_{x x}$ and $\sigma_{z z}$. The $y y$ and $z z$ normal stress are identical to each other, this applies to both their kinetic and configurational parts and $\sigma_{y y}$ is not explicitly shown here. This agreement implies that the flow (which is in the $z$-direction) does not affect either of the perpendicular normal stress components visibly. The fact that the kinetic and the configurational parts of the normal stress profiles oscillate around the same average value is a consequence of the temperature and density of the fluid and is not the case in general (see Figure 7.19). The kinetic normal stresses are all equal and can be expressed in terms of number density $n$ and temperature $T: \sigma_{i i}^{K}=n T$ for each direction $i$ [376]. The configurational stress profiles are coupled to density in a more complicated way. A positive configurational stress implies that the few strong repulsive forces dominate the many weaker attractive forces. This can be seen as an effect of the inhomogeneity in the distribution of the atoms. Alternatively, in a perfect crystal lattice without thermal motion, at the same density, the forces would all be in the attractive regime. The oscillations in both parts of $\sigma_{z z}$ are in phase with each other in the center of the channel and become out of phase towards the wall. Furthermore, for 


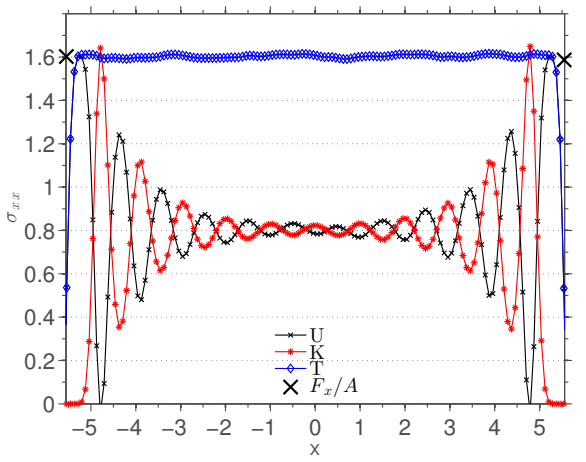

(a)

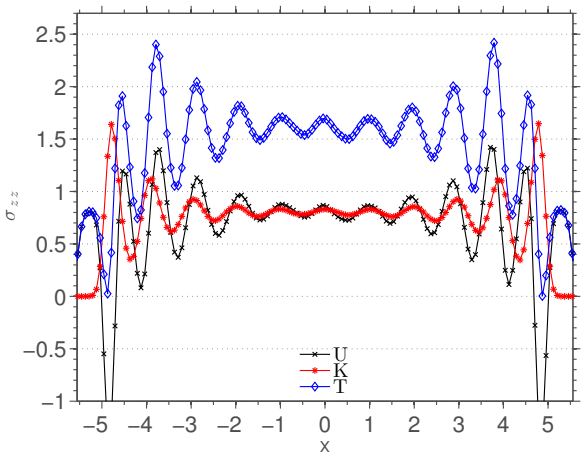

(b)

Figure 7.13: The total $(T)$ normal stress in the $x x$ - (a) and $z z$-direction (b), decomposed into its kinetic $(K)$ and configurational $(U)$ contribution.

the configurational profile, it can be seen that the peak closest to the wall is lower than the adjacent peak, the minimum being even negative, which corresponds to attractive forces.

These observations can be understood better by looking at the interactions between atoms near the wall. A distinction can be made between interactions within a dense layer and interactions between atoms in adjacent layers. The former type of interactions is mostly oriented in the $y$ - $z$-plane, whereas the latter type of interaction has a larger contribution in the $x$-direction due to the directions of the forces. Also the typical interaction lengths are not the same for these two types of interactions, due to a difference in the distribution of atoms within and perpendicular to the layers. The distribution of the atoms in the layers nearest to the walls is strongly influenced by the properties of the walls, which in turn has a major influence on the stress profile. Due to the many factors and the strong nonlinear interaction forces, more study is required in order to get a quantitative understanding of the stress profiles in a strongly confined fluid. This is not pursued in the present work.

Since the fluid is confined in $x$-direction and has a streaming velocity only in the $z$-direction, while the $y$-direction is neutral, the only non-zero shear stresses are $\sigma_{x z}=\sigma_{z x}$, equal due to the symmetry of the stress tensor. The shear stress, shown in Figure 7.14, follows a linear trend with superimposed oscillations near the walls. These oscillations are much less pronounced than those in the normal stresses. Similar to $\sigma_{x x}$, the shear stress profile for a continuum fluid is restricted by the conservation equation of linear $z$-momentum. By integrating this momentum conservation equation 


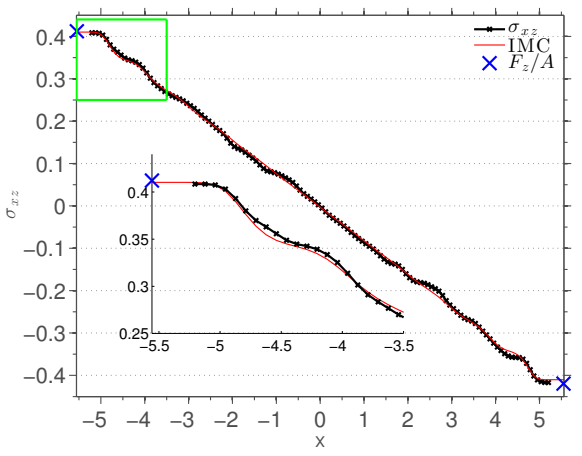

(a)

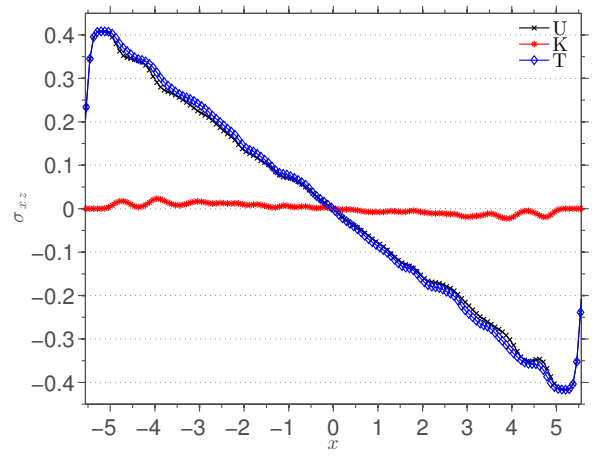

(b)

Figure 7.14: Shear stress across the channel compared to the integrated momentum conservation equation (IMC, Eq. (7.38) ) and decomposed into its kinetic and configurational part. The inset in (a) zooms in on the left near-wall region. The nominal stress along the walls is shown on the left and right side as $\times$. The linear fit to the bulk regime (not shown) gives $\sigma_{x z}=-0.0784 x$.

(IMC) [86, a profile can be calculated to validate the shear stress

$$
\sigma_{x z}(x)=-f \int_{0}^{x} n\left(x^{\prime}\right) d x^{\prime}
$$

Figure 7.14 shows that the shear stress profile obtained from Eq. 7.38 is very close to the measured shear stress data. Also the tangential force on the walls divided by the area of the walls are in agreement with the local shear stress at the walls. We have also looked at the contributions of kinetic and configurational shear stress. The kinetic shear stress is known to be small compared to the configurational part [88, 95, 301, as confirmed by our data as shown in Figure 7.14(b)

\section{Transport properties}

Figure 7.15 shows the shear stress as a function of strain rate across the channel. Nonlinearities appear in the near-wall region, which indicate departure from Newtonian behavior. In the bulk, the negative ratio between the local shear stress and strain rate is a measure for the shear viscosity. The figure shows that this simple constitutive assumption is not valid away from the center of the channel (as discussed in Section 7.4.2, since the shear stress and strain rate sometimes have the same sign due to local extrema in the streaming velocity, this would correspond to a negative shear viscosity according to the Newtonian constitutive relation. A meaningful local scalar 


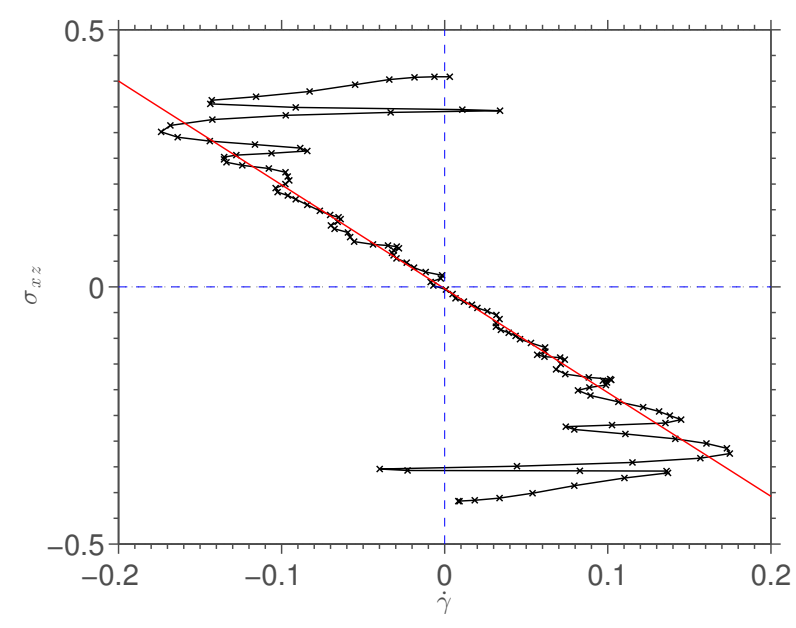

Figure 7.15: Shear stress as a function of displacement strain rate across the channel. The linear fit corresponds to Newtonian behavior in the bulk, there the negative slope (fitted as 1.95) of the line is a measure for the shear viscosity.

shear viscosity cannot be calculated with a Newtonian constitutive relation in these regions.

Figure 7.16 shows the viscosity $\eta=\sigma_{x z} /\left(\epsilon_{x z}^{l i n} / \Delta t\right)$ calculated with the displacement averaging method and the objective viscosity $\eta^{D}=\sigma^{D} \cos (2 \Delta \phi) / s$. The profiles are in good agreement and show strong oscillations across the channel and an increasing trend near the wall. This is to be expected, since the shear rate approaches zero very close to the wall, whereas the shear stress has its maxima near there. The viscosity profiles also show non-physical extrema in the center of the channel, caused by the fact that the denominators in both expressions are close to zero in the center of the channel. Despite this practical inconvenience, an average viscosity in the center region can be calculated as ratio of linear least-square fits of the shear stress and displacement rate profiles, respectively. This approach is not applicable for the objective viscosity since this profile cannot be given as the ratio of two linear profiles. Taking the average of the viscosity in the bulk region directly, leads to a much too high value due to a numerical inaccuracy around the center of the channel, where the strain rate and the shear stress tend to zero, as was also noted by Todd and Evans [367]. Alternatively, the slopes of $\sigma^{D} \cos (2 \Delta \phi)$ and $s=|\dot{\gamma}|$ can be fitted for $-2.06<x<0$ and $0<x<$ 2.06 individually. This way, the fit of the objective viscosity is done in the left and right half of the bulk region separately. The average objective viscosity is fitted as $\left(\eta^{D}\right)^{f i t}=\left(\sigma^{D} \cos (2 \Delta \phi)\right)^{f i t} /(s)^{f i t}=2.06$. Since both viscosities are so close, we will 


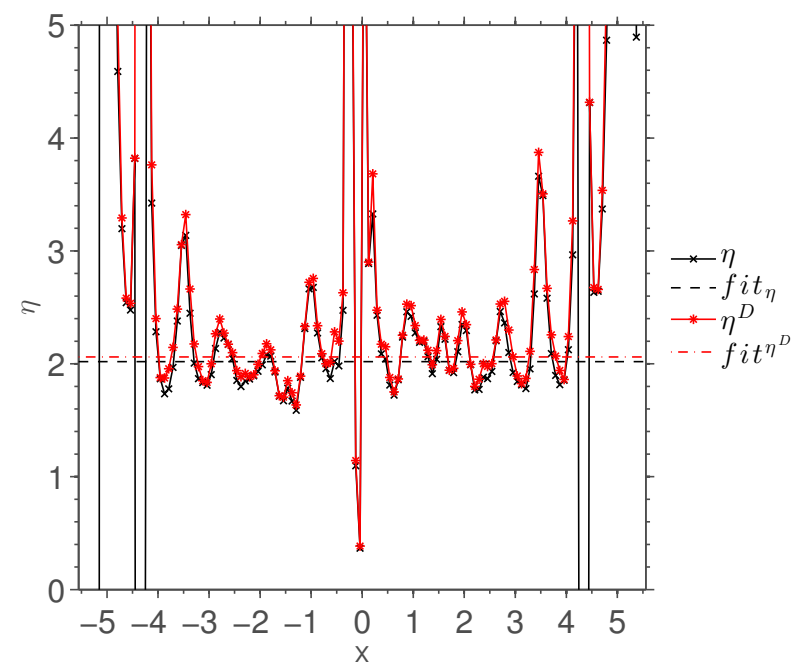

Figure 7.16: Viscosity $\eta$ calculated with the displacement averaging method (Eq. (7.20), shown as a function of $x$. The viscosity is fitted in the bulk region as 2.02 (slope in Figure 7.15). Furthermore, the objective viscosity $\eta^{D}$ (Eq. 7.29 ) is shown. The slopes of $\sigma^{D}$ and $s=|\dot{\gamma}|$ are fitted in the left and right half of the bulk region, giving an average objective viscosity of 2.06 .

not distinguish between them below.

\section{Influence of temperature}

Systems with temperatures $T=0.4,0.6,0.8$ and 1.0 are studied, where $T=1.0$ corresponds to a temperature $\mathrm{k}_{B} T^{*}=121 \mathrm{~K}$ for argon. The body force on the atoms is $f=0.1$, while the density and the channel width are $\rho=0.8$ and $W=11.1$ respectively, as before. Nosé-Hoover thermostats are locally applied near the walls in order to achieve a constant temperature profile across the channel, see subsection 7.4.1 for details. An almost constant temperature profile is obtained for each simulation. The profiles that correspond to temperatures $T=0.6,0.8$ and 1.0 show a slight increase in temperature towards the center of the channel and small fluctuations superimposed on the constant trend.

Figure 7.17 shows the streaming velocity and strain rate profiles across the channel. The velocity profile of the system with temperature $T=0.4$ indicates a solid (i.e., the streaming velocity fluctuates around zero across the channel). Freezing of strongly confined fluids was studied by Ma et al. [340] and by Cui et al. [377, whereas, we focus on liquid systems and do thus not discuss these data further. The simulations 


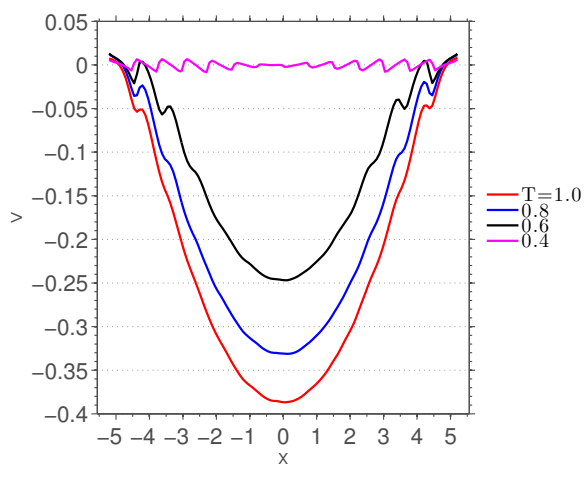

(a)

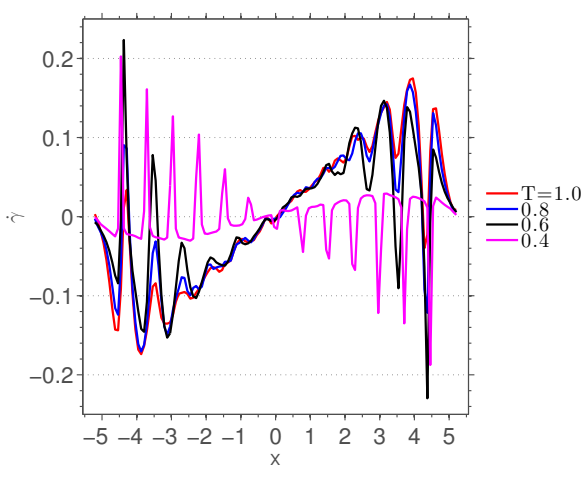

(b)

Figure 7.17: Streaming velocity (a) and strain rate (b) across the channel at different temperatures.

with a temperature $T \geq 0.6$ show velocity profiles similar to the one discussed in Section 7.4.3. Two effects of the temperature can be observed: First, the magnitude of the streaming velocity profile increases with an increasing temperature. Second, the oscillations in the profile are less pronounced in the profiles that correspond to a higher temperature. Each of the velocity profiles for temperatures $T \geq 0.6$ show clear oscillations close to the wall, but the higher the temperature, the faster these oscillations make place for only a bending of the velocity profile, with fewer local extrema. The strain rates in the bulk are quite close for the different temperatures ( $T=0.6,0.8$ and 1.0). The three profiles show clearly that the structures in velocity (and thus in strain rate) close to the wall are more pronounced in the systems with a lower temperature.

The density profiles in Figure 7.18 show two qualitatively different types of behavior. Figure 7.18(a) shows three density profiles that are typical for a strongly confined liquid. Each of them shows strong oscillations near the wall that decrease towards to center. The magnitude of the oscillations decreases with increasing temperature. The profile shown in Figure 7.18(b) corresponds to the lowest temperature $T=0.4$. As the temperature drops below a critical value, the argon atoms form a fixed dense lattice (solid-like phase) attached to the walls, leaving a small open space in the center of the channel where single atoms occasionally move around (vapor-like phase), so that the system is not homogeneous anymore in $y$ and $z$-directions. Due to the high average density, the solid dominates most of the channel, as can be seen from the density profile. We have found that similar phenomena occur for wider channels, a larger vapor region arises in the center, while most atoms in the systems stick to the sides of the 


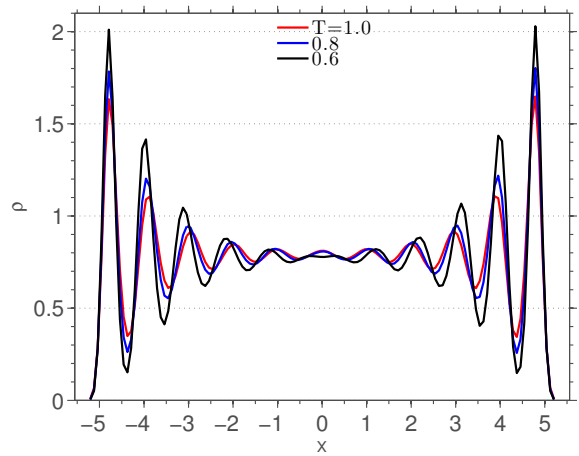

(a)

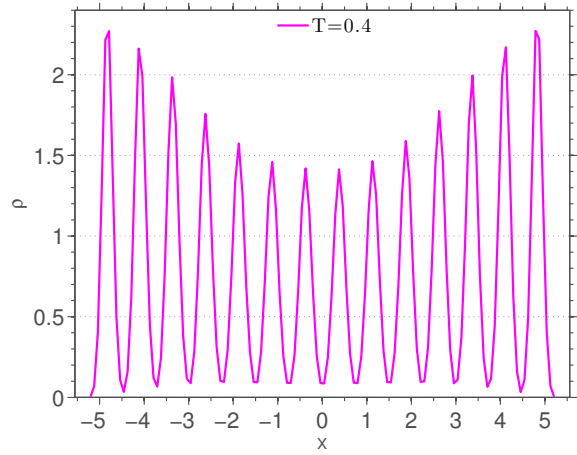

(b)

Figure 7.18: Density profiles for fluid (a) and solid (b) across the channel at different temperatures. The average density in the system is the same for each of the simulations.

channel and arrange in the same lattice as the walls. This is not studied further in the present work.

The normal stress $\sigma_{x x}$ profiles are constant across the channel, similar to Figure $7.13(\mathrm{a})$. The values of the stress and the average kinetic $\sigma_{x x}^{K}$ and configurational $\sigma_{x x}^{U}$ part (averaged over the bulk region shown in Figure 7.9 are shown in Figure 7.19. A linear least-squares fit of the average kinetic stress shows that the average kinetic stress scales approximately linearly with temperature, as is strictly true in case of equipartition. The configurational stress $\sigma_{x x}^{U}$ increases non-linearly with an increase in temperature. This quantity follows from the $x$-components of the force and distance vectors between atoms. Due to the strong non-linearity of the Lennard-Jones potential, the configurational stress has a non-linear relation to the distances between atoms. Slightly smaller distances (in the repulsive regime) can lead to extremely high forces and thus very large positive stress. If the temperature increases, the atoms vibrate faster and the minimum distances that occur are smaller. Hence, the repulsive forces become larger while the attractive forces remain less affected. If the temperature is small enough $T \leq 0.6$ at a density of $\rho=0.8$, there are too few strongly repulsive forces in order to compensate the many attractive forces; hence, the normal stress is negative. This negative normal stress can be sustained in a strongly confined fluid, but would not be thermodynamically stable in a bulk fluid. Similarly, Long et al. 305. observed positive and negative average normal stresses by varying the channel width at a fixed temperature.

Figure 7.20 shows that the shear stress $\sigma_{x z}$, as opposed to the normal stress, does 


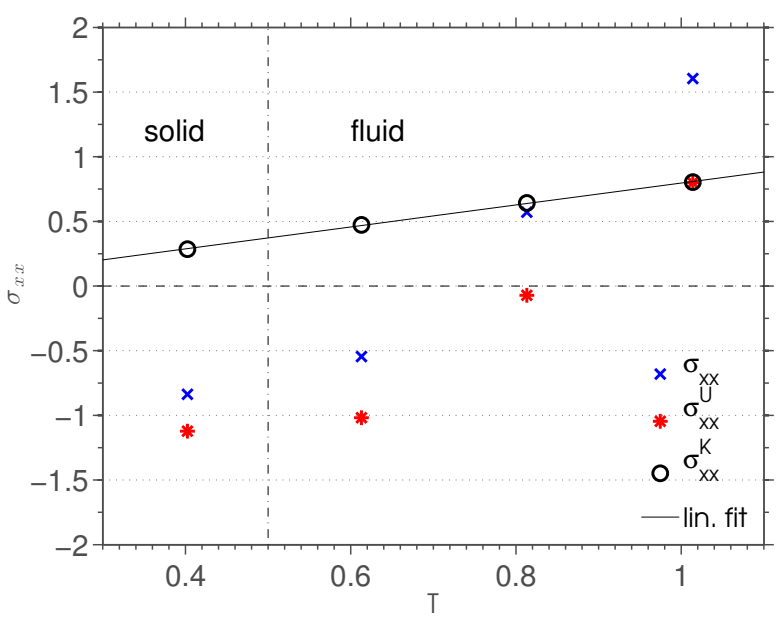

Figure 7.19: Average normal stress $\sigma_{x x}$ in the bulk, against the average temperature in the bulk. The kinetic stress $\sigma_{x x}^{K}$ is fitted with a linear profile given by $n T$. Different regions can be distinguished in the diagram, differing in phase (separated by the vertical dotted line) and in the compressive or attractive nature of the normal stress.

not change much with temperature. This is mostly because the kinetic part of the shear stress is negligible compared to the configurational part, for each of the temperatures and since Eq. (7.38) is independent of $T$, while $\rho$ depends only weakly on $T$. The magnitude of the oscillations in the shear stress decreases slightly with an increasing temperature, similar to the magnitude of the oscillations in density. The non-linearities in the shear stress profiles of liquid systems decay significantly towards the center of the channel, while the shear stress profile of the solid system shows strong oscillations across the whole channel, with only a small decay in magnitude towards the center of the channel. This observation is consistent with the density profiles of the same simulations in Figure 7.18

The viscosity profiles at different temperatures are shown in Figure 7.21. Only the profiles for temperatures $T \geq 0.6$ are shown, since the strain rate profile for $T=0.4$ fluctuates around zero. The shear viscosity profiles do not scale strongly with a change in temperature. However, the structures in the profiles increase with a decrease in temperature, resulting in a slightly higher average viscosity in the bulk.

\section{Influence of body force}

The influence of body force (that acts in the negative $z$-direction) on several physical quantities is studied here. Body forces of $f=0.02,0.05,0.1,0.2$ and 0.3 are compared, 


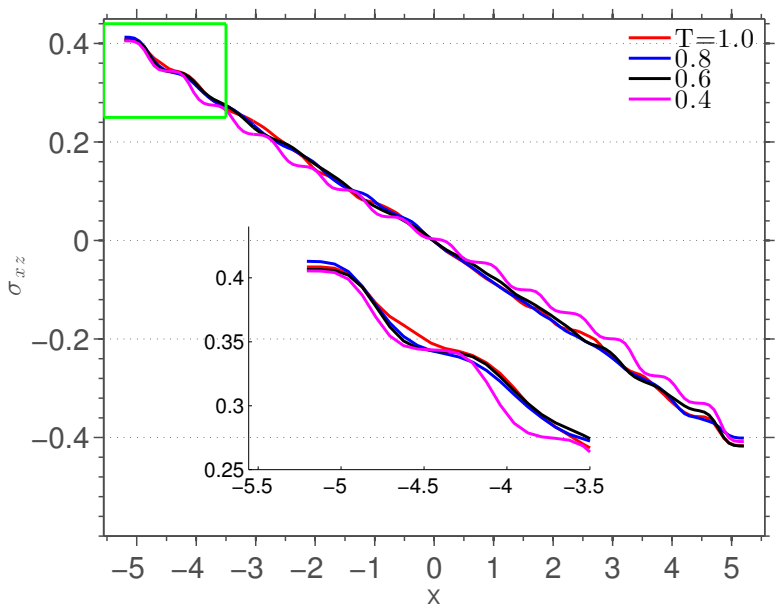

Figure 7.20: Shear stress $\sigma_{x z}$ profiles at different temperatures.

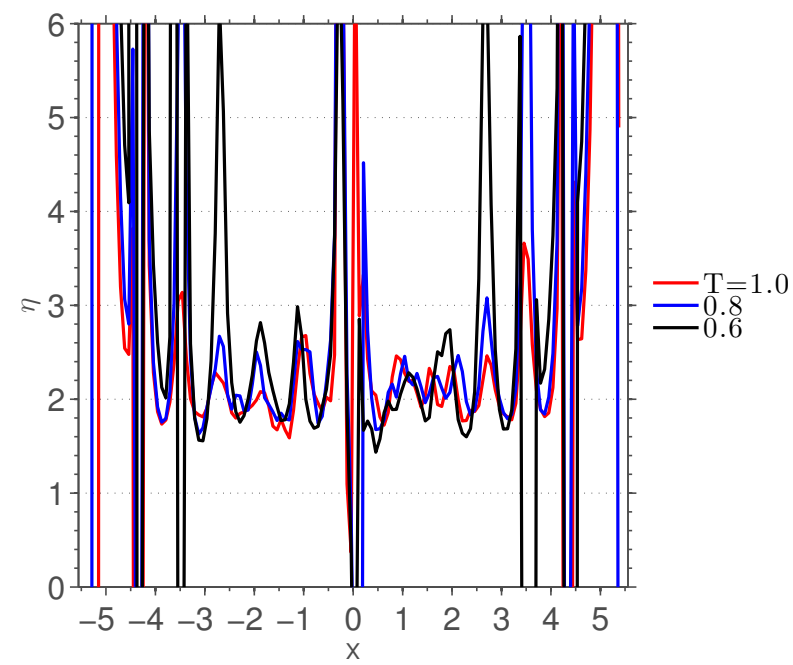

Figure 7.21: Shear viscosity profiles at different temperatures $T \geq 0.6$, calculated with Eq. 7.20$)$. 


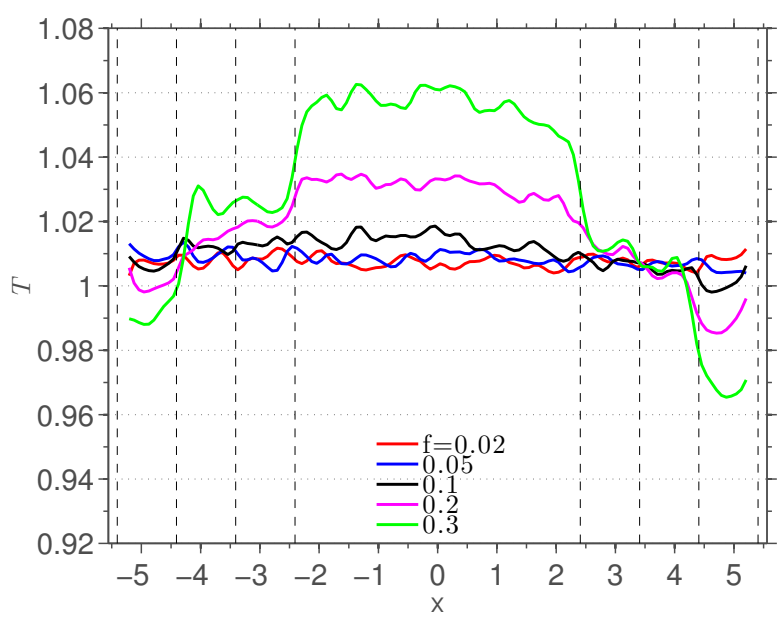

Figure 7.22: Temperature profiles at different body forces with local Nosé-Hoover thermostats applied near the wall, where the vertical dashed lines indicate the 3 layers that are thermostatted on each side.

while the temperature, density and channel width are $T=1.0, \rho=0.8$ and $W=11.1$, respectively. A reduced force $f=1.0$ corresponds to a force of $f^{*}=4.9 \cdot 10^{-12} \mathrm{~N}$ for argon. However, considering the mass of the atoms, this seemingly small force on the atoms is many orders of magnitude larger than, for example, a standard gravitational force on the atoms would be.

The averaged quantities presented in this section are, as specified earlier, calculated by smoothing the data with a smoothing length of $w=0.1$ and averaging over 5000 snapshots over a period of time of 5000. The simulation time step is $d t=0.001$ and $M=134$ data points are used across the channel, so that the points are separated by $\Delta x=W / M \approx 0.08$.

The obtained density profiles are not notably dependent on the body force, and are thus not explicitly shown here.

Figure 7.22 shows that the temperature fluctuates around $T \geq 1.0$ across the channel for body forces $f \leq 0.1$. As the body force increases to $f \geq 0.2$, the average temperature in the bulk region (which is not thermostatted) increasingly increases from the constant target temperature across the channel. Thus, the local thermostats are not sufficient when the body force is too large, as discussed in Binder et al. 368.

The streaming velocity and the strain rate profile that are shown in Figure 7.23 for different body forces show a very similar behavior and are almost symmetric as expected. Close to the walls, small wiggles can be seen in the velocity profile. In 


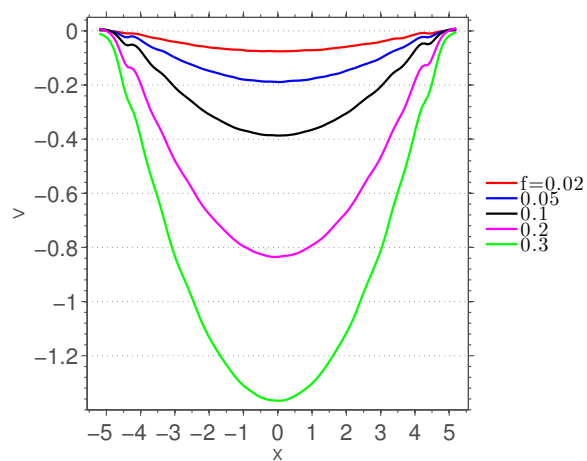

(a)

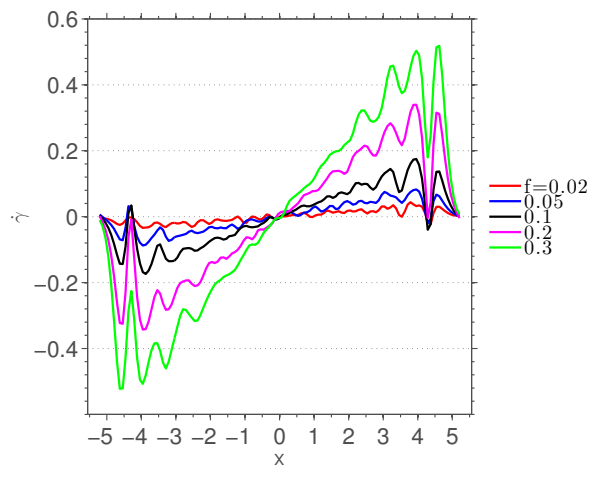

(b)

Figure 7.23: Streaming velocity (a) and strain rate (b) profiles at different body forces.

the center of the channel, the velocity profile is approximately quadratic, apart from small statistical fluctuations. The magnitude of the oscillations and the quadratic trend of the streaming velocity increase linearly with the body force. The strain rate profile shows a clear oscillatory behavior also further away from the walls. Both the magnitude of the trends and the oscillations in the streaming velocity and the strain rate profiles increase with an increasing body force. Scaling of $\dot{\gamma}$ by $f$ leads to a collapse of the curves, with the exception of the magnitude of the oscillations very close to the walls, these are relatively larger in the case of small body forces.

Figure 7.24 shows the normal stress $\sigma_{x x}$ and shear stress $\sigma_{x z}$ across the channel. The normal stress profiles are almost the same for body forces from $f=0.02$ to 0.1 , whereas the profiles that correspond to body forces $f \geq 0.2$ show strongly increasing stress with increasing $f$. This is due to the configurational stress, since, as shown in Section 7.4.3. the dynamic stress contribution scales linearly with temperature, while the configurational stress has a strongly non-linear relation to temperature. From the profiles that correspond to a constant temperature of $T \approx 1.0$, we observe that the body force does not affect the normal stress $\sigma_{x x}$ much, i.e., the small difference between the normal stress profiles correspond to slight differences in temperature between body forces $f=0.02,0.05$ and 0.1 . Since the stress is isotropic in the center of the channel (due to symmetry), each of the normal stress profiles (i.e., $\sigma_{x x}, \sigma_{y y}$ and $\sigma_{z z}$ ) oscillates around the same average value. Hence, each of the normal stress profiles is independent of the body force at a constant temperature.

The shear stress $\sigma_{x z}$ is shown in Figure 7.24(b) Fine structures are seen in the near wall region for each profile, superimposed on a linear trend. The slopes of the trends scale linearly with the body forces as Eq. (7.38) indicates. Also the oscillations 


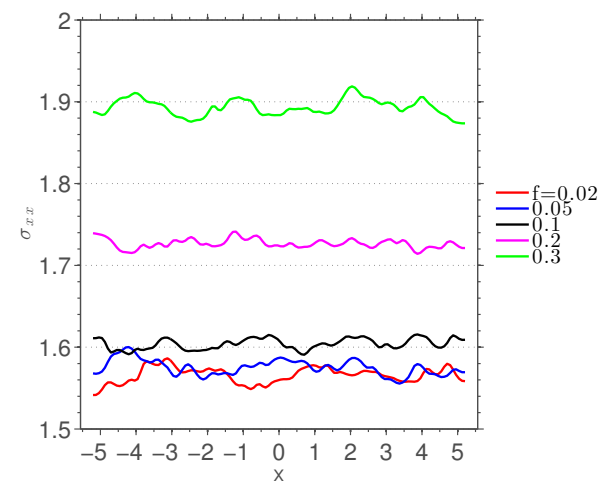

(a)

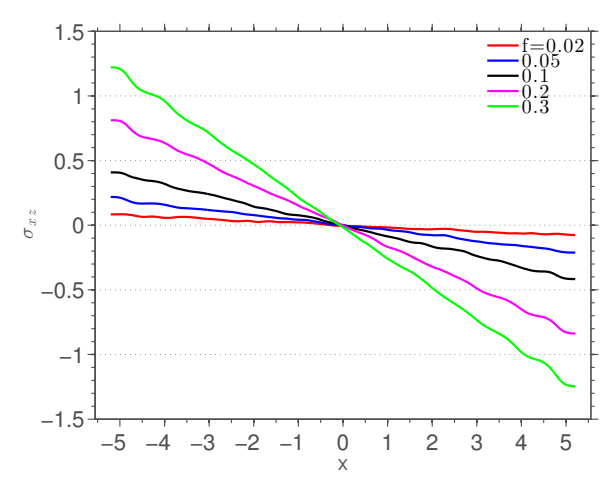

(b)

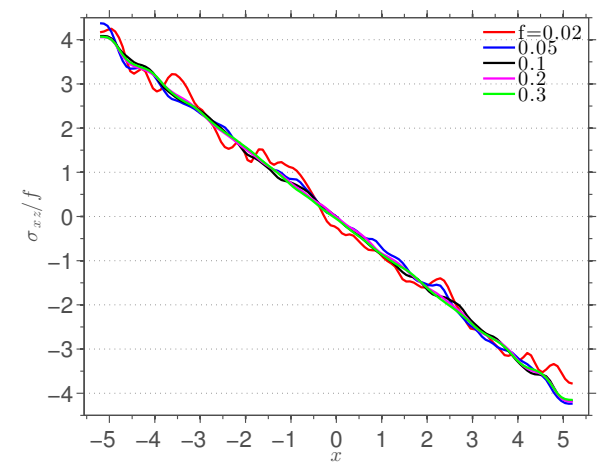

(c)

Figure 7.24: Normal stress $\sigma_{x x}$ (a), shear stress $\sigma_{x z}$ (b) and shear stress scaled by body force (c).

in the shear stress profiles are more pronounced for higher body forces, in agreement with Eq. 7.38). The shear stress profiles divided by the corresponding body forces results in a collapse of profiles onto each other (shown in Figure 7.24(c), including the magnitude of the near-wall variations, as consistent with the independence of the density profiles on $f$. The scaled profiles clearly show the increasing noise level with decreasing body force.

The viscosity profiles are shown in Figure 7.25. Since both strain rate and shear stress scale linearly with the body force, the Newtonian shear viscosity $\eta$ in the bulk is found to be practically independent of the body force. However, the fluctuations in viscosity grow with a decrease in body force, since the strain rate is more sensitive to noise than the shear stress profile. The fact that the viscosity is not (strongly) 


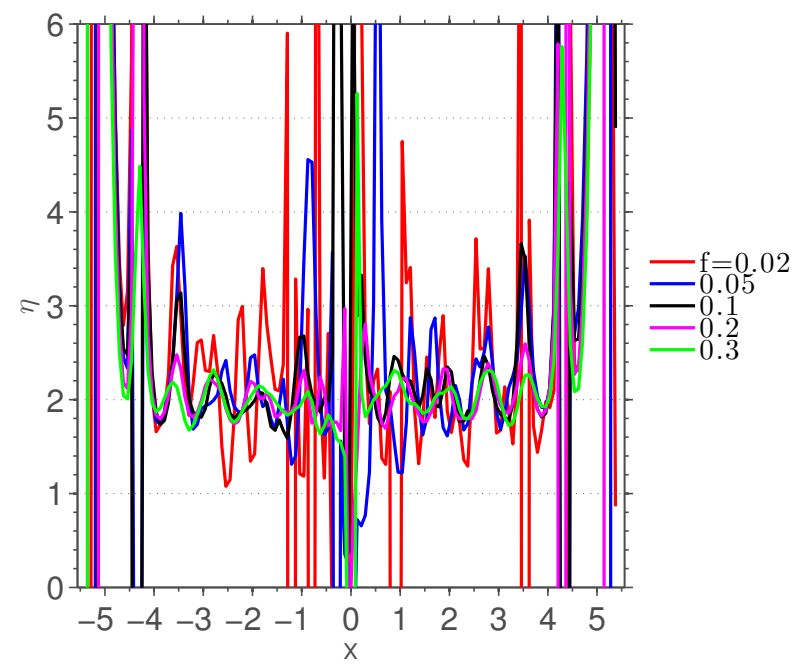

Figure 7.25: Shear viscosity profiles at different body forces, calculated with Eq. (7.20).

dependent on body force indicates that the viscosity is a possible function of the density, temperature and confinement of the fluid, rather than the external driving force.

\subsubsection{Constitutive model}

In Section 7.4.2, the deviatoric stress tensor is expressed in terms of three variables, $\lambda_{1}, \lambda_{2}$ and $\alpha \equiv \phi_{\sigma}$. We study here the relation between these variables and some of the macroscopic fields that were presented in Section 7.4.3, like density $\rho$, strain rate $\dot{\gamma}$ and temperature $T$.

\section{Density, velocity and temperature}

The oscillations in the density profile are a direct consequence of the layering of the atoms between the two confining walls. The density profile oscillations depend weakly on the temperature of the fluid, while the average density does not. With an increase in temperature, the layering of the atoms, and thus the oscillation amplitude in density, decreases. The locations of the layers are practically invariant to changes in temperature since they are determined mostly by the walls, except for very lo $T$, where crystallization begins to set in. Furthermore, the density profile is independent of the body force and thus not related to the flow-dependent quantities such as streaming velocity or strain rate, in the bulk, for the regime of parameters studied here. 
The streaming velocity is non-zero in the direction of the body force and zero (on average) in the directions perpendicular to the body force. The profile is approximately quadratic away from the walls and shows oscillations near the walls that are correlated to the layering: large velocity gradients $\dot{\gamma}$ occur between the layers, whereas, the layers themselves do not shear internally which leads to small $\dot{\gamma}$. The magnitude of the oscillations in the streaming velocity profile increases with increasing body force and decreases with an increasing temperature of the fluid.

Since the temperature is controlled locally, it is difficult to conclude from the temperature profiles how temperature is related to other quantities. However, from the fact that the local thermostatting did not suffice when the body forces become too large, we can conclude that the temperature of the fluid increases with the body force, since a higher strain rate leads to a faster generation of heat.

\section{Stress}

As Eq. (7.13) shows, the stress tensor is directly related to fluctuation velocities and interactions between pairs of atoms. While we do not study the quantitative behavior of the kinetic and configurational stresses explicitly here, we summarize our observations made in Section 7.4.3. The kinetic part of the normal stress profiles are given by $\sigma_{i i}^{K}=n T$ for each direction $i$, as observed also by Rowlinson and Widom [376]. Since the kinetic stress is thus isotropic, the deviatoric stress tensor is fully determined by the configurational stress contribution. The stress tensor can be written as: $\boldsymbol{\sigma}=\left(n T+\frac{1}{3} \operatorname{tr}\left(\boldsymbol{\sigma}^{U}\right)\right) \mathbf{I}+\boldsymbol{\sigma}^{D}=p \mathbf{I}+\boldsymbol{\sigma}^{D}$, where $p$ and $\boldsymbol{\sigma}^{D} \equiv\left(\boldsymbol{\sigma}^{U}\right)^{D}$ will be discussed further in Section 7.4.4. It is far from obvious if and how each of the configurational stress components are related to other measured quantities. Since the oscillations in the $y y$ - and $z z$-components are different in period and phase from the oscillations in density, these profiles are not directly proportional to density alone. While a full understanding of the normal stresses is beyond the scope of this paper, we conclude that the $\sigma_{x x}$ normal stress is not oscillating and thus not directly dependent on the body force, streaming velocity or strain rate (due to momentum conservation equilibrium conditions), for the parameters used. Furthermore, the normal stress $\sigma_{x x}$ increases with increasing temperature, see Figure 7.19, as seen in both its kinetic and configurational stress contributions. Studying the interactions between atoms within a layer and interactions between different layers is paramount to acquiring a good microscopic understanding of the behavior of the stresses, but goes beyond the scope of this study.

\section{Shear viscosity}

We already mentioned in Section 7.3.2 that the shear viscosity of an inhomogeneous fluid cannot be accurately described by a scalar Newtonian constitutive relation. This 
means that the local shear viscosity is not just a linear combination of the local shear stress and strain rate, but can be a more complicated relation, for example one that contains an additional field or one that is nonlocal in space and time. The possibility of a spatially nonlocal shear viscosity is considered in several studies [293, 294, 329]. Finding a suitable kernel or other expression for shear viscosity for confined fluids is still an open problem and is not studied here.

The model that is proposed in this work involves two eigenvalues of the deviatoric stress and an orientation, which should be the complete set of macroscopic variables that have to be taken into account. Considering the full viscosity tensor on the other hand would be the right approach, to describe the layered structures near the wall, but also in the bulk zone. This, however, would blow up the complexity too much as compared to the rather simple approach proposed here.

\section{The isotropic and deviatoric stress}

In Section 7.4.4, we discussed the decomposition of the stress into its isotropic and deviatoric part. The pressure $p$ is the isotropic part of the stress tensor. The pressure contains the kinetic stress and the average normal configurational stress. The kinetic stresses are linearly coupled to density and temperature, whereas, the normal configurational stresses have a more complicated dependency on density and temperature. Hence, the pressure is dependent on density and temperature via both the kinetic and configurational contribution. The pressure is, like each of the normal stresses, not directly dependent on the body force.

To further analyze and understand the stress behavior and the relation between shear stress and strain rate, we carry out an eigenvalue analysis of the deviatoric stress tensor $\boldsymbol{\sigma}^{D}=\boldsymbol{\sigma}-p \mathbf{I}$. The deviatoric stress tensor can be expressed in terms of its eigenvalues and orientation angle, as shown in Eqs. 7.31 and 7.32). However, the relation between the eigenvalues of the deviatoric stress tensor and for example the density, shear rate and viscosity of the fluid is unknown 6 The maximum $\lambda_{1}$, intermediate $\lambda_{2}$ and minimum $\lambda_{3}$ eigenvalues, i.e., principal deviatoric stresses, are obtained and plotted as a function of the position in Figure $7.26(\mathrm{a})$ and the ratio between them in Figure 7.26(b), The figure shows that the intermediate principal deviatoric stress $\lambda_{2}$ oscillates around zero, whereas the maximum $\lambda_{1}$ and minimum $\lambda_{3}$ eigenvalues show oscillations superimposed on a linear trend, increasing from the center to the walls. These linear trends follow from the shear stress, since the normal stresses oscillate around a constant value.

Note that the sum of the eigenvalues of the deviatoric stress tensor equals zero.

\footnotetext{
${ }^{6}$ Note that the existence of a local relation is not guaranteed. The possibility of a non-local viscosity kernel, which is suggested in Refs. [293, 329], is not studied here.
} 


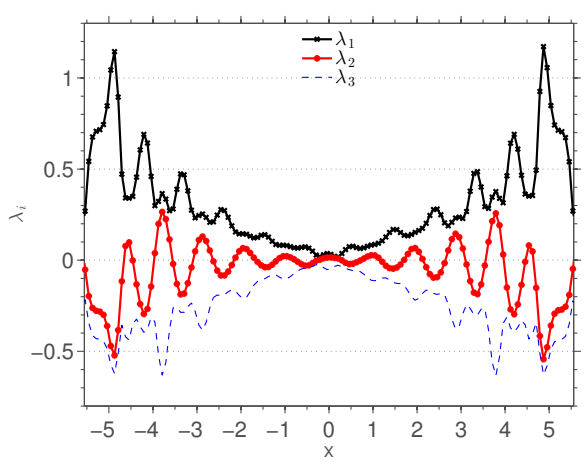

(a)

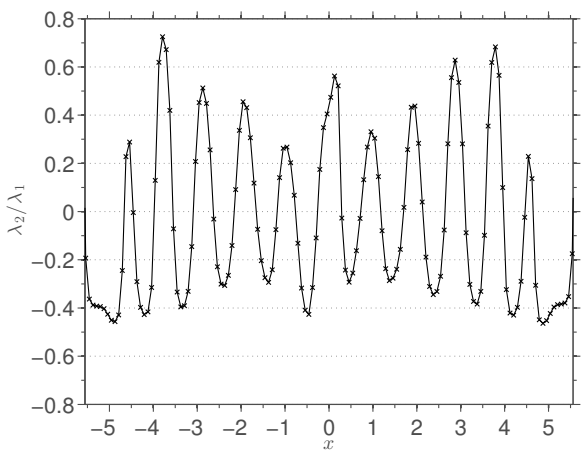

(b)

Figure 7.26: Principal deviatoric stresses and their ratio across the channel, for a simulation with a body force of $f=0.1$, an average density of $\rho=0.8$ and a temperature $T=1.0$. Note than $\lambda_{3}=-\left(\lambda_{1}+\lambda_{2}\right)$ is exactly fulfilled and $\sigma_{y y} \approx \lambda_{2}$ is true within $1 \%$.

Therefore, one has two independent eigenvalues $\lambda_{1}$ and $\lambda_{2}$, the third one $\lambda_{3}=-\left(\lambda_{1}+\right.$ $\left.\lambda_{2}\right)$ is expressed in terms of the independent eigenvalues. It was already derived that the following relation applies for the simple shear case

$$
\lambda_{1}+\frac{\lambda_{2}}{2}=\frac{\sigma_{x y}}{\sin \left(2 \phi_{\sigma}\right)}=p S_{D}
$$

While this expression relates the eigenvalues to objective quantities, it is yet unknown how the values of the eigenvalues $\lambda_{1}$ and $\lambda_{2}$ relate to each other or to other fluid properties (such as density, temperature and velocity). The eigenvalues are approximately equal only in the center of the channel, which indicates an isotropic stress only at this location. The stress is anisotropic elsewhere, see Figure 7.27.

The differences between the principal stresses can be used as a measure for the stress anisotropy, in contrast to the more traditional approach where stress anisotropy is expressed in terms of normal stress differences, see Section 7.4.2. We divide stress anisotropy by pressure to make it quantify its relative magnitude. Figure 7.27 shows the stress anisotropy as defined in Eqs. 7.15) and (7.16), very similar, both qualitatively and quantitatively. Note that this may not be the case for other systems, for example when the system is not plane-symmetric and invariant in $y$-direction. Both formulations for the anisotropy are exactly identical if $\lambda_{1}=-\lambda_{3}$ and $\lambda_{2}=0$, see Section 7.3.2. It can be confirmed from the intermediate principal stress $\lambda_{2}$ in Figure $7.26(\mathrm{a})$ that this condition is not met, but nevertheless $S_{D} \approx S_{D}^{*}$, since the extreme values dominate. 


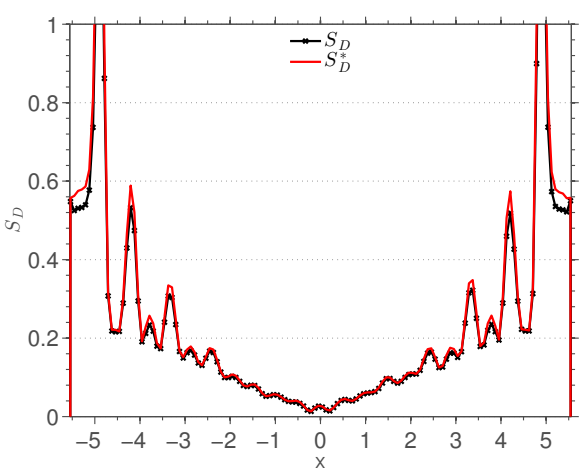

(a)

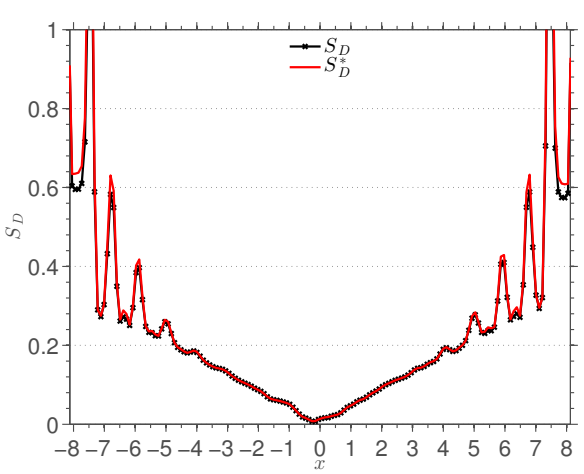

(b)

Figure 7.27: Stress anisotropy across the channel, for a simulation with a body force of $f=0.1$, an average density of $\rho=0.8$ and a temperature $T=1.0$. The channel width is $W=11.1$ in (a) and $W=16.2$ in (b).

Across the whole channel, from the isotropic center, the anisotropy shows a linearly increasing trend towards the walls, resulting in a finite stress anisotropy, also in the bulk region, so that $p S_{D} \propto s=|\dot{\gamma}|$, see Eq. 7.29 . The anisotropy is approximately zero in the center of the channel, however, the anisotropy is slightly larger than zero due to small fluctuations in the stress. The oscillations in the stress anisotropy are limited to a narrow near-wall region of width approximately 3 , resulting in a bulk region of width approximately 5 . We have also looked at the same quantities for a channel of width $W=16.2$ (Figure $7.27(\mathrm{~b})$ ), where we obtain a similar behavior with a bulk region of approximately 10 wide. The peaks in $S_{D}$ increase towards the walls and show two distinct amplitude trends. When comparing Figures 7.9 and 7.27 one can see that the large (small) peaks in $S_{D}$ are correlated with decreasing/small (increasing/large) densities:7. In other words, stress anisotropy is extreme in the lower density slip-planes, while it also reaches relatively smaller maxima within the dense layers. In the same spirit, when comparing Figures 7.10(b) and 7.27, one can relate the large (small) peaks in $S_{D}$ to minima (maxima) in strain rate magnitude $|\dot{\gamma}|$.

The eigenvectors $\left(\mathbf{v}_{i}, i=1,2,3\right)$ that corresponds to the principal deviatoric stresses define the orientation of the principal stress tensor. Since these vectors are mutually orthogonal by definition, we only need to specify two vectors to define the principal orientation. The vector that corresponds to the intermediate principal stress is always aligned with the $y$-axis (within statistical fluctuations). Because the vectors are or-

\footnotetext{
${ }^{7}$ Where increasing or decreasing are defined from the center towards the walls, i.e., $d \rho / d x$ larger or smaller than zero.
} 
thogonal, $\mathbf{v}_{1}$ and $\mathbf{v}_{3}$ lie in the $x$-z-plane, so that only one orientation (of one of these vectors) defines the principal orientation of the stress. Figure 7.28 shows the rotation angle $\alpha$ around the $x$-, $y$ - and $z$-axis for the eigenvector $\mathbf{v}_{1}$ that corresponds to the maximum principal deviatoric stress $\lambda_{1}$. The angles are denoted by the plane in which they are rotated, e.g. $\alpha_{x z}$ is the angle that the vector $\mathbf{v}_{1}$ makes with respect to the $x$-axis rotated in the $x$ - $z$-plane. Figure $7.28(\mathrm{a})$ shows the orientation of the eigenvector $\mathbf{v}_{1}$ for a channel of 11.1 wide, whereas, Figure $7.28(\mathrm{~b})$ corresponds to a channel of 16.2 wide. The figures show that the rotations in the $y$-z-plane and the $x$-y-plane are approximately zero, thus the vector $\mathbf{v}_{1}$ is mainly oriented in the $x$ - $z$-plane and oscillates around an average angle with respect to the $z$-axis. Only in some of the dense layers the vector is not oriented in the $x$ - $z$-plane, but the angle $\alpha_{x z}$ is not visibly affected. The average angle in the $x$ - $z$-plane between the $z$-axis and the vector $\mathbf{v}_{1}$ is $\alpha= \pm 45^{\circ}$, similar to the expected principal stress orientation in a Navier-Stokes, collinear channel flow. The maxima in $\alpha_{x z}$ coincide with the minima in the density profile and the maxima in $\lambda_{1}$, while the minima in $\alpha_{x z}$ correspond to the tiny deviations from the linear trend in Figures 7.26(a) and 7.27. The magnitude of the oscillations in the orientation angle is large relative to the average value and the oscillations decay away from the walls. This decay is more clear in the wider channel (Figure 7.28(b) but relatively weak in the narrower channel (Figure 7.28(a)p.

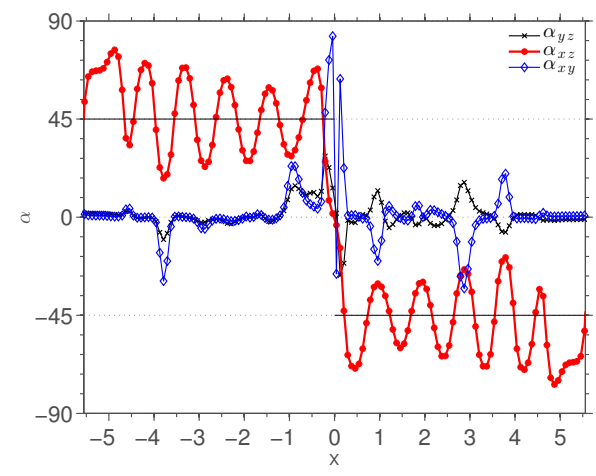

(a)

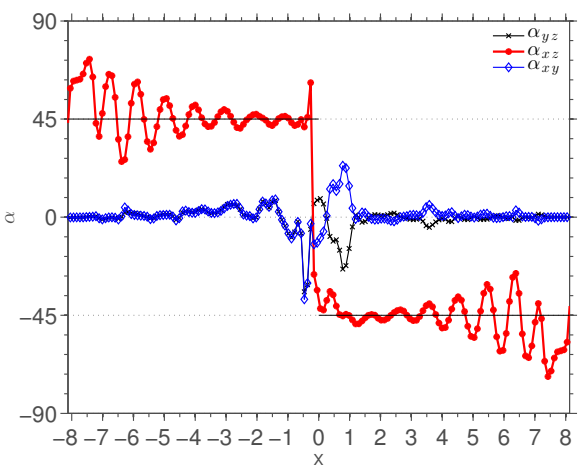

(b)

Figure 7.28: The orientation angle $\alpha$ around each of the Cartesian axes, for (a) $W=$ 11.1, and (b) $W=16.2$. The oscillations in $\alpha_{x z}$ slowly decay towards the bulk. This decay is more pronounced in the wider channel (b).

It was found, for the wide channel, that the cosine of the orientation angle $\phi_{\sigma}$ of the deviatoric stress tensor is proportional to the square root of the magnitude of the oscillations in density. The same applies, for the simple shear case, to the sine of the 
angle difference $\Delta \phi=\phi_{S}-\phi_{\sigma}$ of the deviatoric stress relative to the strain rate tensor. The angle difference is given by

$$
\Delta \phi=c_{\phi} \sin ^{-1}\left(\operatorname{sign}(\Delta \rho) \sqrt{\frac{|\Delta \rho|}{\rho_{a v}}}\right)
$$

where $\Delta \rho=\rho-\rho_{\text {bulk }}$ denotes the oscillations in density, $c_{\phi}$ is a proportionality constant, the term in front of the square root takes care of the sign of the oscillations, while the square-root term takes care of their magnitudes. This expression is not derived from any theory. We know that for a homogeneous sheared fluid (Chapter 5 ) the angle differences is proportional to the vorticity $\omega=\dot{\gamma} / 2$. The dependence of $\Delta \phi$ on the local shear rate $\dot{\gamma}$ has not yet been investigated.

The agreement between Eq. 7.40 and the data is shown in Figure7.29 for $W=$ 11.1 and (b) $W=16.2$. The decay of $\Delta \phi$ is again clearer in the wider of the two channels and cannot be successfully fit for $W=11.1$. The proportionality constants that we found are $c_{\phi}=-0.8$ for $W=11.1$ and $c_{\phi}=-0.5$ for $W=16.2$. Both figures show that the oscillations in the orientation angle are in phase with those in the density profile, except for a small shift very close to the walls. In the middle of the channel, the lagging angle deviates from the smoothly oscillating behavior. This was also observed in Figure 7.28 and is caused by the fact that the deviatoric stress tensor is approximately zero in the middle of the channel.

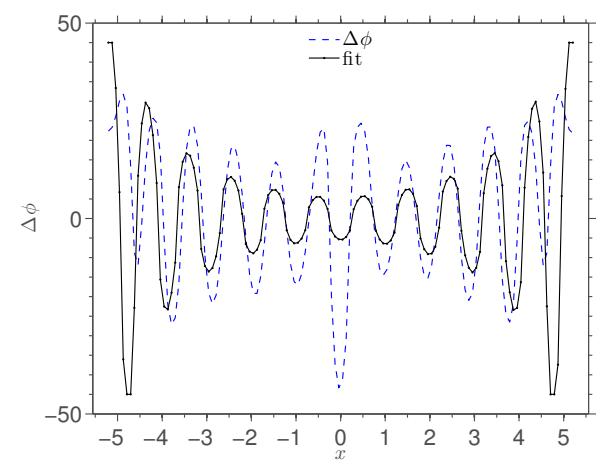

(a)

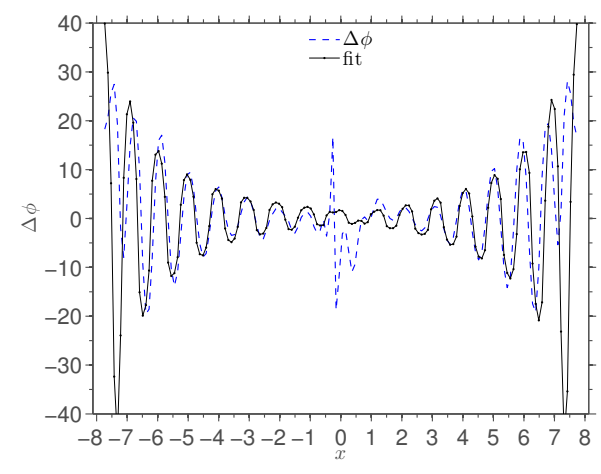

(b)

Figure 7.29: The angle difference $\Delta \phi$ fitted with Eq. 7.40 , for (a) $W=11.1$, and (b) $W=16.2$. 


\subsection{Conclusions and summary}

This chapter has given an introduction to the literature and methods related to simulating fluids confined in a nanochannel. In such narrow channels, the atoms arrange in layers near the wall, such that the properties of the fluid become a function of the position across the channel. Homogeneity in the directions parallel to the walls is usually assumed. In order to check the validity of this assumption, we looked at the two-dimensional density and velocity field in the plane of flow. It has been shown that, very close to the wall, the density is inhomogeneous parallel to the wall. Atoms are drawn to the locations of local minimum potential energy, and they can escape these locations again due to their kinetic energy. The fluid freeze to the wall if atoms do not have sufficient kinetic energy to escape the potential energy-well. This situation is not studied here. The streaming velocity profile shows no clear inhomogeneity in the direction parallel to the walls. Therefore, the assumption of homogeneity in these directions can be justified in most cases, while not strictly true very close to the wall. A full three-dimensional analysis might be the key to a better understanding of the stresses near the walls.

The variation of density, velocity, stresses and other quantities across the channel depends on a number of simulation parameters and methods used. Furthermore, the roughness of the wall and the method of heat removal are known to have a considerable effect on the slip between fluid and wall. We have discussed some of these parameters and performed a study of planar Poiseuille flow of argon in nanochannels of about $4.0 \mathrm{~nm}$ width, driven by a constant body force. The influence of the system-walls, the channel-width, the body force and the fluid temperature on the rheological properties have been studied. The goal is to better understand the layering in strongly confined fluids.

Furthermore, the anisotropy and the non-Newtonian flow rheology in this system were quantified and explained in the framework of an objective constitutive model that is applicable to a wide range of systems. We mainly focused on the challenging properties of strongly confined fluids, however, several other special cases have also been discussed.

Given the rich complexity of the fluid close to the wall, the over-simplified classical concepts (like fluid-wall slip) have to be thought over and were not explicitly investigated in the derivation of the model presented here. Having a constitutive model at hand that works in the bulk as well as in the layered region, one can describe the boundary layer with the more advanced model, and at the same time resemble the classical Newtonian fluid model far away from the wall. This approach is different from hybrid modeling, where two different methods (e.g., MD and CFD) are coupled [41. In our approach, the multi-scale aspects and increased complexity close to the 
walls should be taken into account by an advanced anisotropic continuum model which contains the (classical) bulk fluid as a limit case.

The atoms in a confined liquid arrange themselves in layers near the wall. This phenomenon appears to be independent of the magnitude of the body force and is not affected much by the fluid temperature. The layering of the fluid strongly affects, for example, the streaming velocity, i.e., the layers slip along each other.

We have quantified the degree of non-Newtonian flow-behavior: For this, a decomposition of the deviatoric stress is introduced, which is based on eigenvalue analysis. The deviatoric stress tensor is rotated to its principal orientation, where it can be described by only two independent eigenvalues and by an orientation angle. The angle in the $x$ - $z$-plane, between the $z$-axis and the major (positive) principal deviatoric stress orientation, shows strong oscillations across the channel - even rather far away from the walls - indicating that anisotropy is decaying more slowly than the density oscillations due to layering. The two independent eigenvalues $\lambda_{1}$ and $\lambda_{2}$ are both displaying similar oscillations as the density close to the walls. Further away from the walls, $\lambda_{1}$ decays with a linear trend towards the center, whereas the oscillations proceed into the system for $\lambda_{2}$. The ratio between the independent eigenvalues $\lambda_{2} / \lambda_{1}$ shows similar strong oscillations as the principal stress orientation - even rather far away from the walls. A constitutive model is propose ${ }^{8}$ that takes both stress-deviator contributions into account as well as the orientation of the deviatoric stress eigen-system. We found that none of them can be neglected and neither can isotropy or collinearity of stress and strain be assumed anywhere in the channel.

Due to the enormous complexity of the stresses in an inhomogeneous system, more study is required in order to get a better understanding of the layering in the configurational stress fields near the walls. Complementing the macroscopic picture presented here, in what way the walls exactly influence the stress profiles can be better understood by looking closer at the microscopic structure, for example the interaction between pairs of atoms in the same layer and in adjacent layers. Either type of structure influences the configurational normal stresses and can thus be seen in the pressure and the deviatoric stresses. Only if these effects are understood, it will be possible to gain more insight in the relations between inhomogeneous and anisotropic stresses and other quantities for strongly confined fluids.

The constitutive model could be extended with additional quantities like the anisotropy of structure, velocity slip and thermal slip to contribute to a more complete picture. These extensions of the model were not considered in the present study and a better understanding of the dependence of slip on the wall properties would be required

\footnotetext{
${ }^{8}$ This model was proposed earlier than the model in Chapter 5 expressing the inhomogeneous stress tensor for a confined fluid in terms of the four non-Newtonian model parameter (Eqs. 55.57)- 5.60) can hopefully increase our understanding of the inhomogeneous stresses further.
} 
before including this property in the model. 


\section{8}

\section{Conclusions and recommendations}

The work presented in this thesis has made contributions primarily to the following (related) areas: Firstly, the calculation of material properties and their dependence on the state point and on the velocity field. Calculations have been performed with various equilibrium and non-equilibrium molecular dynamics methods. Secondly, nonNewtonian phenomena have been quantified and combined with an objective constitutive framework to predict the non-Newtonian pressure tensor for confined and unconfined fluids.

\subsection{Homogeneous fluids}

In Chapter 5 equilibrium stress autocorrelation functions (SACF) have been used for the calculation of a range of equilibrium fluid properties, such as: the zero-shear rate viscosity, the zero-shear rate first normal stress coefficient, a relaxation time and the infinite-frequency shear modulus. We have studied the SACF, and the fluid properties that can be extracted from it, over a range of densities. It was found that the shear relaxation modulus of a dilute atomic fluid can be accurately described with a simple functional form consisting of a Gaussian relaxation for short times plus a single exponential decay for long times. In dense fluids, the interactions between atoms are much more frequent and each interaction may be related to a sequence of previous events. Consequently, the SACF for dense fluids has a more complicated functional that is governed by various coupled relaxation mechanisms. To our knowledge, there was no data on the density dependence of the zero-shear rate first normal stress coefficient. We have found a surprising density dependence, with a minimum coefficient around density $\rho=0.52$ and an increasing coefficient towards the dilute and dense 
fluid limits. Which mechanisms are responsible for this trend is not fully understood yet, although the increasing coefficient in the dense-fluid region is likely to be related to the increasing memory effect in a dense viscoelastic fluid.

Non-equilibrium molecular dynamics (NEMD) simulations of a homogeneous atomic fluid under shear flow, planar elongational flow and a combination of shear and elongational flow have been unified consistently with an objective model over a wide range of strain rates. A model was presented that predicts the pressure tensor for a nonNewtonian bulk fluid for any homogeneous planar flow field. It was found that the eigenvalues of the deviatoric pressure tensor satisfy a simple functional form that can be expressed in terms of the viscosity, the viscoelastic lagging angle and an out-offlow-plane pressure anisotropy. Consequently, the non-Newtonian pressure tensor for a planar flow can be decomposed into a hydrostatic pressure, a tensor that describes the deviatoric stresses in the plane of flow, and a tensor that quantifies the anisotropy perpendicular to the plane of flow. It was found that three of these non-Newtonian quantities were completely objective and can be expressed as a function of the magnitude of the strain rate tensor. Interestingly, the non-equilibrium increase in pressure shows the same scaling with the deformation rate as found for the magnitude of the out-of-flow-plane anisotropy, while shear viscosity follows a different functional behavior. The viscoelastic lagging angle, on the other hand, is proportional to the vorticity of the fluid, which is directly related to the velocity gradient. The lagging angle divided by the magnitude of the vorticity collapses onto a single profile that is, once again, a function of the magnitude of the strain rate tensor.

The objective constitutive framework that we have presented for steady planar flows of simple atomic fluids has been verified for a single state point. A more extensive study is currently being undertaken to provide a more complete picture of how the non-Newtonian quantities scale with the magnitude of the strain rate tensor at different state points. Some results have been presented in Appendix B to confirm the scaling of the four parameters with the shear rate at different state points. Conclusions drawn from this data have not yet been used to extend and improve our model. The data suggests that the functional form that predicts $\Delta \phi$ is different at low densities. This would have to be studied in more detail in order to extend our model to be valid for a wide range of state points. Furthermore, our current model expresses the pressure tensor in terms of non-Newtonian phenomena. These phenomena find their origins in the microstructure of the fluid. It is currently being investigated how the non-Newtonian pressure tensor is related to structural quantities of the fluid, such as the order tensor or the pair distribution function. Another interesting extension of the model could be achieved by studying the transient pressure tensor in its objective form. The pressure tensor in a viscoelastic fluid not only depends on the current defor- 
mation rate, but also on the deformation history of the fluid. We have shown that the transient shear stresses for large deformation rates are not well-described with linear viscoelastic theory and an overshoot can form in the stress profile due to structural rearrangement in the fluid. Therefore, the non-equilibrium pair distribution function would be an indispensable component for an extension of our model to account for the evolution of pressure. Taking these steps is advised for gaining a good understanding of non-Newtonian stresses in simple atomic fluids. More complicated materials, such as polymer melts or granular materials, can complicate the picture further.

The transient-time correlation function (TTCF) method was used in Chapter 6 for the calculation of the pressure tensor and the viscosity of fluids subjected to various constant planar flows. This method is more efficient than direct averages of NEMD simulations when the deformation rate is sufficiently small, and thus offers a potentially suitable method for direct comparisons between computational results and data measured from 'real' systems. Rather than such a comparison, the treatment of TTCF in this work mainly focussed on applying the method to various planar-flow NEMD simulations. We have successfully applied TTCF to calculating transient stress profiles and the viscosity for a simple atomic fluid under planar mixed flows. A comparison between TTCF results and averaged NEMD data showed that, if the deformation rate decreases, the statistical uncertainty of the TTCF method increases less than that of a direct NEMD average that is calculated from the same amount of data. This signifies the use of TTCF for comparing computational results to experimental results, in which the deformation rates are typically many orders of magnitude smaller than those feasible in most NEMD simulations. Since most real systems contain molecular fluids, we have applied TTCF to fluids consisting of short chain molecules, deformed under shear flows, planar elongational flows and planar mixed flows. We have found for these relatively simple molecules that translational, rotational and vibrational degrees of freedom can be identified in the SACF. The overshoot in the transient profile is only observed if enough degrees are active and becomes bigger as the chain length increases, while its location is not strongly dependent on the chain length.

The TTCF calculations for atomic and molecular fluids have been shown to agree with direct NEMD averages, but a comparison to real experimental results has yet to be made. Despite the fact that TTCF is more efficient at low shear rates than direct averaging of NEMD data, these calculations are still very computationally expensive for molecular fluids due to their long relaxation times. A direct comparison is most feasible for fluids consisting of small molecules without long-range electrostatic interactions. Examples of such fluids are alkanes, as frequently used in the petrochemical industry. Using TTCF for accurate calculations of steady-state stresses may be too computationally expensive for large molecules, but it could be interesting to use TTCF 
to calculate the transient stresses at short times. The sudden increase and overshoot in the transient stresses could have important consequences for the life-expectancy of pumps or valves.

\subsection{Confined fluids}

The study of state variables in inhomogeneous fluids is far more complicated than that in bulk fluids. This is not only the case because local quantities vary as a function of the distance from the wall near a solid-fluid interface, but also because these variations depend on the choice of numerous parameters involved with the interactions between the fluid and the solid. Consequently, a model that relates the state variables to each other will also depend on the design choices that were made. Extensive parameter studies would be required to acquire a complete overview of the various dependencies.

In Chapter 7 we have studied, for a Lennard-Jones fluid confined between two atomistic walls, the dependency of local state variables on the average fluid densities, fluid temperatures and values for the body force that drives the fluid. Special attention was given to the eigenvalues of the deviatoric stress tensor and to the tensor components that correspond to the directions normal and parallel to the walls, as well as their decomposition into a kinetic and a configurational part. The simulation results have been used to formulate a tensorial model in which the local stress tensor is expressed in a number of objective scalar quantities, such as the pressure, anisotropy and tensor orientation. Each of them are again complicated functions of the position in the channel. The relation of the stress-related quantities to other state variables is still an open question, especially very close to the wall.

In addition to studying time and space-averaged profiles, a closer look at the dynamics of atoms and the structure of the fluid near the wall shows that the fluid atoms closest to the (frozen) solid walls temporarily become part of the lattice structure. Atoms are drawn to the locations of local minimum potential energy, and they escape these locations again if they possess sufficient kinetic energy (which they already have or is transferred via interactions). If the temperature of the fluid is sufficiently low, atoms will spend a longer time in the sites directly next to the wall and the fluid will (partially) freeze to the wall, leaving a dilute gas further away from the walls. An analysis of the two-dimensional density field of a confined fluid has confirmed that the preferred locations of atoms near a solid wall causes the fluid very close to the wall to be inhomogeneous in directions normal and parallel to the walls, while further from the walls the inhomogeneity is only observed in the perpendicular direction.

The variations in the configurational stress profiles in the direction perpendicular to the walls are in phase with those in the kinetic stress profiles. For the directions 
parallel to the wall, the variations in the configurational and kinetic stress are in phase in the center of the channel, while they shift out of phase near the wall. The inhomogeneity in the directions parallel to the walls is often considered to be negligible, but might be a key element in gaining understanding and finding a quantitative description of stress profiles near a solid interface. This would have to be investigated. It would be interesting to study the relation between various two- or three-dimensional stress, density and other fields, very close to a wall, to answer the question if homogeneity parallel to the walls may be assumed. It is expected that the influence of the thermostatting approach on these multi-dimensional fields is large very close to the wall and we advise the use of thermostatted walls, as is consistent with experimental situations.

\subsection{Outlook}

While the macroscopic properties of bulk fluids can often be measured experimentally, MD simulations provide insight in the structural properties of a fluid, that cannot yet be achieved via experimental studies. Furthermore, computer simulations offer more flexibility in scanning a range of properties than, often more expensive and time-demanding, experiments. As long as this remains the case and computers keep becoming more powerful, it is likely that $\mathrm{MD}$ will continue to be a valuable method for the study of bulk fluids. A major part of these studies will involve dense polymer melts, bio-materials, pharmaceuticals or ionic liquids, driven by the potential profits for industrial companies and the quest for a better fundamental understanding.

For confined-fluid simulations, a promising future lies ahead. The number of nanofluidic applications grows rapidly and present-day computers are becoming more capable to deal with the computational requirements of simulating some of these systems. In recent years, a lot of computational studies have been devoted to, for example, confined polymeric fluids, fluid flow through or around carbon nanotubes and flow between graphene sheets. These systems are studied a lot because of their industrial relevance. Yet, even in much simpler systems, such as a Lennard-Jones fluid in a rectangular channel, many open questions remain. For example, expressing local stress values in terms of density, shear rate and other quantities is an open problem for inhomogeneous fluids. Shear stress might be related to shear rate via a non-local viscosity kernel. Promising work has been done to find kernels that can correctly predict the non-local stress for confined fluids. Deriving these kernels is still an open question and so far there are no theoretical frameworks available to compute these. Computational studies will hopefully contribute to solving some of the open problems associated with confined fluids on the nanoscale. 
8.3. OUTLOOK 


\section{Derivation of the SLLOD equations of motion}

Newton's second law of motion states that the acceleration of an object depends on its mass and on the sum of all forces acting on the object. This law of motion can be written in terms of two coupled first-order differential equations

$$
\begin{aligned}
\dot{\mathbf{r}}_{i} & =\mathbf{v}_{i}, \\
\dot{\mathbf{v}}_{i} & =\frac{\mathbf{F}_{i}^{T}}{m_{i}},
\end{aligned}
$$

where $\dot{\mathbf{r}}_{i}$ and $\dot{\mathbf{v}}_{i}$ are the evolution of the laboratory position and velocity vectors of atom $i, m_{i}$ is its mass and $\mathbf{F}_{i}^{T}$ is the total force acting on the atom.

Our goal is to superimpose a linear streaming velocity profile onto the fluid instantaneously at time $t=0$. This profile can be expressed in terms of the velocity gradient as $\mathbf{u}(\mathbf{r}) \Theta(t)=\mathbf{r} \cdot \nabla \mathbf{u} \Theta(t)$, where $\Theta(t)$ is the Heaviside step function.

We can split the laboratory velocity of an atom into a thermal part $\mathbf{c}_{i}$ and the contribution of the streaming velocity $\mathbf{u}\left(\mathbf{r}_{i}\right)$. Doing so for Eq. A.1 gives

$$
\dot{\mathbf{r}}_{i}=\mathbf{c}_{i}+\mathbf{u}\left(\mathbf{r}_{i}\right) \Theta(t)=\mathbf{c}_{i}+\mathbf{r}_{i} \cdot \nabla \mathbf{u} \Theta(t),
$$

The left hand side of Eq. A.2 can be rewritten in the same way as

$$
\begin{aligned}
\dot{\mathbf{v}}_{i} & =\dot{\mathbf{c}}_{i}+\frac{d}{d t}\left(\mathbf{r}_{i} \cdot \nabla \mathbf{u} \Theta(t)\right) \\
& =\dot{\mathbf{c}}_{i}+\dot{\mathbf{r}}_{i} \cdot \nabla \mathbf{u} \Theta(t)+\mathbf{r}_{i} \cdot \nabla \mathbf{u} \delta(t)
\end{aligned}
$$

Combining Eqs. A.2, A.3 and A.5 gives

$$
\dot{\mathbf{v}}_{i}=\dot{\mathbf{c}}_{i}+\mathbf{c}_{i} \cdot \nabla \mathbf{u} \Theta(t)+\mathbf{r}_{i} \cdot \nabla \mathbf{u} \cdot \nabla \mathbf{u} \Theta(t)+\mathbf{r}_{i} \cdot \nabla \mathbf{u} \delta(t)=\frac{\mathbf{F}_{i}^{T}}{m_{i}}
$$


The total force on atom $i$ is a combination of internal forces $\mathbf{F}_{i}$ and a fictitious (i.e., unphysical) driving force $\mathbf{F}_{i}^{E}$. Multiplying both sides with the atomic mass $m_{i}$ and writing the product of mass and peculiar velocity as the peculiar momentum $\mathbf{p}_{i}=m_{i} \mathbf{c}_{i}$ results in

$$
\dot{\mathbf{p}}_{i}+\mathbf{p}_{i} \cdot \nabla \mathbf{u} \Theta(t)+m_{i} \mathbf{r}_{i} \cdot \nabla \mathbf{u} \cdot \nabla \mathbf{u} \Theta(t)+m_{i} \mathbf{r}_{i} \cdot \nabla \mathbf{u} \delta(t)=\mathbf{F}_{i}+\mathbf{F}_{i}^{E}
$$

Using Eqs. A.3 and A.7, we can rewrite the equations of motion (Eqs. A.1) and A.2) as

$$
\begin{aligned}
\dot{\mathbf{r}}_{i} & =\frac{\mathbf{p}_{i}}{m_{i}}+\mathbf{r}_{i} \cdot \nabla \mathbf{u}_{i} \Theta(t) \\
\dot{\mathbf{p}}_{i} & =\mathbf{F}_{i}+\mathbf{F}_{i}^{E}-\mathbf{p}_{i} \cdot \nabla \mathbf{u} \Theta(t)-m_{i} \mathbf{r}_{i} \cdot \nabla \mathbf{u} \cdot \nabla \mathbf{u} \Theta(t)-m_{i} \mathbf{r}_{i} \cdot \nabla \mathbf{u} \delta(t) .
\end{aligned}
$$

These equations are still identical to Newton's equations of motion, expressed in terms of the positions and the peculiar momenta. The next step is to quantify the fictitious driving force vector that leads to the correct velocity profile as well as the correct rate of energy dissipation.

Tuckerman et al. 378, presented their $g$-SLLOD equations of motion by assuming that the fictitious force is zero, such that the equations of motion are

$$
\begin{aligned}
\dot{\mathbf{r}}_{i} & =\frac{\mathbf{p}_{i}}{m_{i}}+\mathbf{r}_{i} \cdot \nabla \mathbf{u}_{i} \Theta(t), \\
\dot{\mathbf{p}}_{i} & =\mathbf{F}_{i}-\mathbf{p}_{i} \cdot \nabla \mathbf{u} \Theta(t)-m_{i} \mathbf{r}_{i} \cdot \nabla \mathbf{u} \cdot \nabla \mathbf{u} \Theta(t)-m_{i} \mathbf{r}_{i} \cdot \nabla \mathbf{u} \delta(t) .
\end{aligned}
$$

In the absence of a fictitious driving force, the fluid is incorrectly driven by the deforming periodic boundary conditions, which is not supposed to interfere with the fluid. These boundary conditions are devised such that the shape of the simulation cell evolves with the imposed streaming motion of the fluid. Therefore, driving the fluid by means of the periodic boundary conditions produces approximately the desired streaming motion of the fluid, even without a fictitious force. However, the atoms are driven only at the boundaries, rather than homogeneously. Furthermore, the $g$-SLLOD equations of motion do not lead to the correct rate of energy dissipation and are thus incorrect, which is proven in Ref. [145].

The evolution of positions and peculiar momenta of individual atoms are dependent not only on the external driving force, but also on their position and momenta relative to other atoms. In order to quantify the correct driving force, we need to look at the evolution of the whole system. Therefore, the external driving force that produces and maintains the desired velocity field can be obtained as follows

$$
\sum_{i=1}^{N} \dot{\mathbf{v}}_{i}=\sum_{i=1}^{N} \frac{\mathbf{F}_{i}^{E}}{m_{i}} .
$$


Note that the interatomic forces cancel each other out, such that the sum over them vanishes, only the external force contributes to the zero wave vector component of the momentum density as shown by Daivis and Todd [145.

Using Eq. A.7 and the fact that $\sum_{i=1}^{N} \mathbf{p}_{i}=0$ and $\sum_{i=1}^{N} \dot{\mathbf{p}}_{i}=0$, Eq. A.12 can be rewritten as

$$
\sum_{i=1}^{N} \mathbf{F}_{i}^{E}=\sum_{i=1}^{N}\left(m_{i} \mathbf{r}_{i} \cdot \nabla \mathbf{u} \delta(t)+m_{i} \mathbf{r}_{i} \cdot \nabla \mathbf{u} \cdot \nabla \mathbf{u} \Theta(t)\right)
$$

Since the equations of motion are spatially homogeneous (i.e., the dynamics of each atom is described with the same equations of motion, regardless of its position or momentum), dividing both sides of Eq. A.13 by the number of atoms in the system is the same as eliminating the summation completely. Finally, we obtain the external force vector on atom $i$ as

$$
\mathbf{F}_{i}^{E}=m_{i} \mathbf{r}_{i} \cdot \nabla \mathbf{u} \delta(t)+m_{i} \mathbf{r}_{i} \cdot \nabla \mathbf{u} \cdot \nabla \mathbf{u} \Theta(t) .
$$

The two terms in this force vector are responsible for superimposing and maintaining the flow field, respectively. Note that the last term, required to maintain the flow, vanishes for example in the case of a shear flow velocity gradient. In that case, no force is needed to maintain the flow after it is imposed. This is not the case for extensional flows or planar mixed flow, in which case these situations need a force to maintain the constant flow.

If we substitute Eq. A.14 into the momentum evolution equation (Eq. A.7) some terms cancel out against each other. The equations we arrive at are known as the SLLOD equations of motion

$$
\begin{aligned}
\dot{\mathbf{r}}_{i} & =\frac{\mathbf{p}_{i}}{m_{i}}+\mathbf{r}_{i} \cdot \nabla \mathbf{u} \\
\dot{\mathbf{p}}_{i} & =\mathbf{F}_{i}-\mathbf{p}_{i} \cdot \nabla \mathbf{u}
\end{aligned}
$$

These equations have successfully been used over the last three decades for the simulations of various homogeneous flows. 


\section{Validation of our objective}

\section{model for the pressure tensor}

The model that was presented in Chapter 5 was initially based on a single state point. Simulation data for a WCA fluid at different state points has been produced with the purpose of validating, improving and extending our model. The state points correspond to a range of different densities $\rho=0.36,0.52,0.68,0.84,0.92$, while the temperature is kept constant at $T=1.0$. It was confirmed that also for these state points the quantities are dependent on the magnitude of the strain rate tensor $s$, rather than on the type of planar flow. Consequently, we have simulated simple shear flow $s=|\dot{\gamma}|$ for a range of shear rates $\dot{\gamma}=0.1 \ldots 2.5$. The other simulation details are identical to those listed in Section 5.3 .

Figure B.1 shows the scaling of the model parameters for five different densities, the corresponding equilibrium quantities and fitting parameters are given in TableB.1. The viscosity, pressure dilatancy and out-of-shear-plane pressure anisotropy are accurately described by the function forms that were suggested in Chapter5. This shows that the proportionality of $\Delta p$ and $a$ with the shear rate are independent of the state point. The Carreau relation captures the shear thinning viscosity accurately for each of the state points, but the order of the normalized viscosity profiles (Figure B.1(b) shows that the shear-thinning behavior cannot be predicted by a simple scaling of the density. Finally, the data suggests that the functional form that predicts the lagging angle $\Delta \phi$ shows a different shear-rate dependency for the different densities. This would have to be studied in more detail in order to extend the model in Chapter 5 to be valid for a wide range of state points.

Furthermore, it is interesting to note how, in the traditional description of the pressure tensor, the normal stress differences depend on the state point of the fluid. This is shown in Figure B.2 It was mentioned in Chapter 2 that the order of the 
Table B.1: Model parameters for six different state points (SP). SP1: $\rho=0.8442$, $T=0.722$ (data presented in Chapter 5), SP2: $\rho=0.36, T=1.0$, SP3: $\rho=0.52$, $T=1.0, \mathrm{SP} 4: \rho=0.68, T=1.0, \mathrm{SP} 5: \rho=0.84, T=1.0, \mathrm{SP} 6: \rho=0.92, T=1.0$.

\begin{tabular}{c|cccccc} 
& $\mathrm{SP} 1$ & $\mathrm{SP} 2$ & $\mathrm{SP} 3$ & $\mathrm{SP} 4$ & $\mathrm{SP} 5$ & $\mathrm{SP} 6$ \\
\hline$\eta_{0}$ & 2.32 & 0.3002 & 0.4984 & 0.943 & 2.127 & 3.650 \\
$p_{0}$ & 6.3903 & 0.8552 & 1.8799 & 3.8646 & 7.8372 & 11.1475 \\
\hline$c_{p}$ & 0.7492 & 0.0318 & 0.0863 & 0.2211 & 0.5928 & 0.9701 \\
$\eta 1$ & 21.21 & 1.1267 & 0.3924 & 0.5578 & 3.1641 & 16.9037 \\
$\eta 2$ & 0.076 & 0.1804 & 0.1430 & 0.1007 & 0.0961 & 0.1091 \\
$c_{a}$ & 0.1113 & 0.0199 & 0.0308 & 0.0536 & 0.0978 & 0.1310 \\
$c_{\phi}$ & 1.5883 & - & - & - & 0.4869 & 1.1815 \\
$b$ & 0.1824 & - & - & - & -0.0309 & -0.0127
\end{tabular}

normal stresses in a sheared fluid depend on the state point. This is confirmed here by the fact that the first normal stress difference $\mathcal{N}_{1} \equiv P_{y y}-P_{x x}$ can have a positive or a negative sign, while the second normal stress difference $\mathcal{N}_{2} \equiv P_{z z}-P_{y y}$ is negative for all the data shown. 


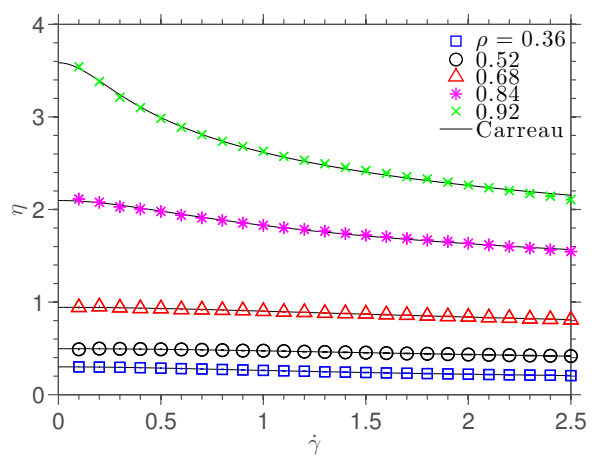

(a)

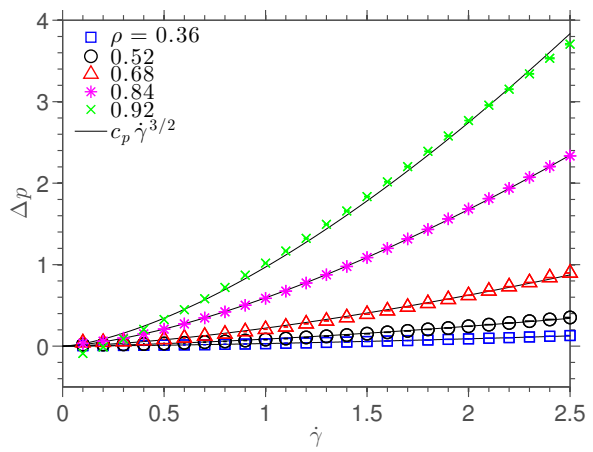

(c)

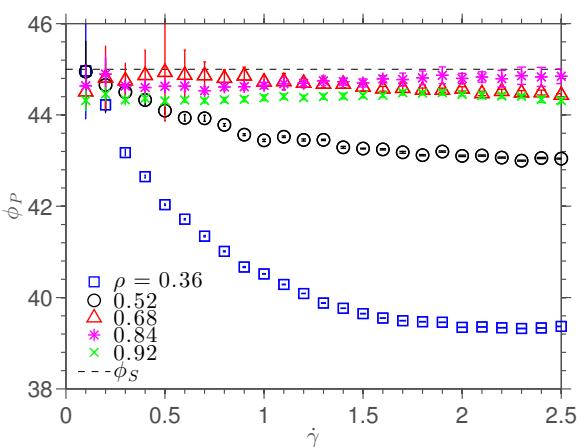

(e)

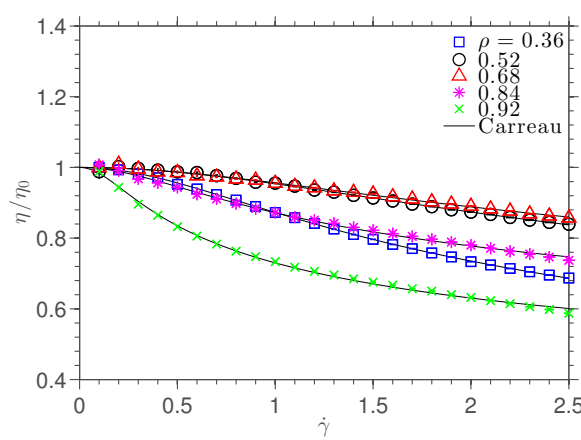

(b)

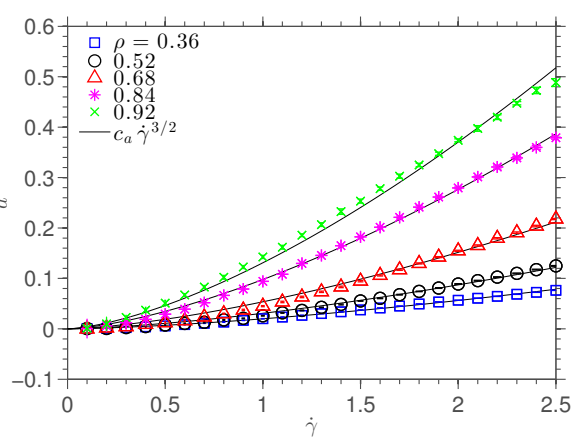

(d)

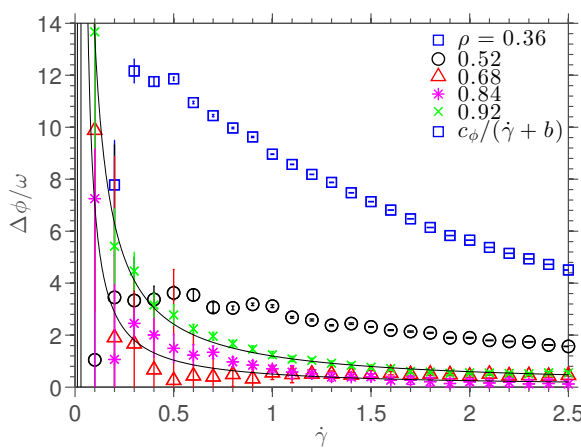

(f) Only the 2 largest densities are successfully fitted

Figure B.1: Model scaling at different densities. 


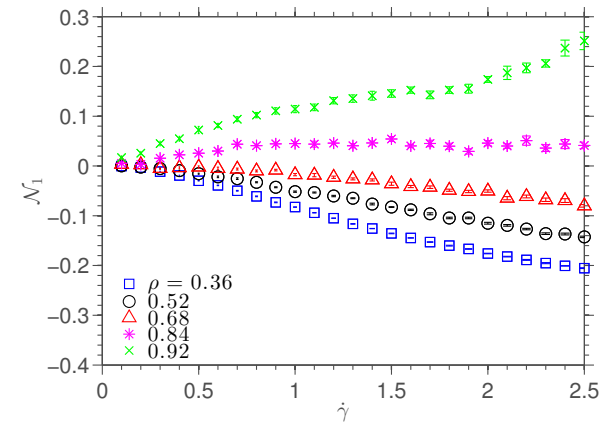

(a)

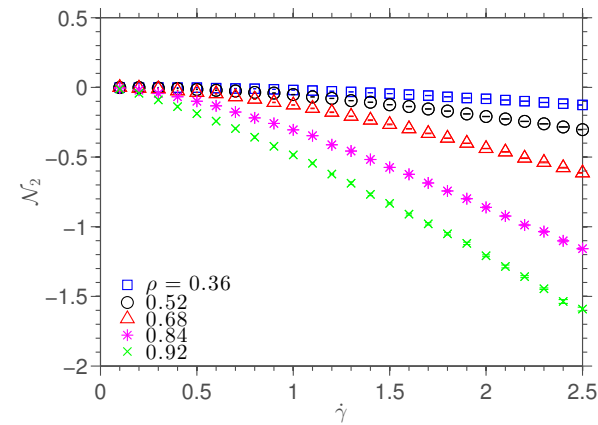

(b)

Figure B.2: The first (a) and second (b) normal stress differences for a WCA fluid under shear flow. Different fluid densities are shown, while the temperature is fixed at $T=1.0$. 


\section{Bibliography}

[1] Richard P. Feynman. There's plenty of room at the bottom: an invitation to open up a new field of physics. Engineering and Science (California Institute of Technology), 23(5):22-36, 1960.

[2] Norio Taniguchi. On the basic concept of 'Nano-Technology'. Proc. Int. Conf. Prod. Eng. Tokyo, Part II, Japan Society of Precision Engineering, pages 18-23, 1974.

[3] Jan Youtie, Philip Shapira, and Alan L. Porter. Nanotechnology publications and citations by leading countries and blocs. J. Nanoparticle Res., 10:981-986, 2008 .

[4] Wikipedia. http://en.wikipedia.org/wiki/Nanotechnology_in_fiction, 2012.

[5] K. E. Drexler. Engines of Creation.: The Coming Era of Nanotechnology. Anchor, 1986.

[6] J. Attali and J. Leggatt. A Brief History of the Future: A Brave and Controversial Look at the Twenty-First Century. Arcade Publishing, 2006.

[7] D. Mijatovic, J. C. Eijkel, and A. van den Berg. Technologies for nanofluidic systems: top-down vs. bottom-up-a review. Lab. Chip., 5(5):492-500, 2005.

[8] W. Sparreboom, A. van den Berg, and J. C. T. Eijkel. Principles and applications of nanofluidic transport. Nature Nanotech., 4:713, 2009.

[9] Mohamed Gad-el-Hak. The fluid mechanics of microdevices-the freeman scholar lecture. J. Fluids Eng., 121(1):5-33, 1999.

[10] Mohamed Gad-el-Hak. Liquids: The holy grail of microfluidic modeling. Phys. Fluids, 17(10):100612, 2005. 
[11] George Karniadakis, Ali Beskok, and Narayan Aluru. Microflows and Nanoflows: Fundamentals and Simulation (Interdisciplinary Applied Mathematics). Springer, 1st edition, 2005.

[12] S. Granick. Motions and Relaxations of confined fluids. Science, 253:1374, 1991.

[13] A. Mukhopadaya, J. Zhao, S. C. Bae, and S. Granick. Contrasting Diffusion and Viscosity in Molecularly-Thin Films. Phys. Rev. Lett., 89:136103, 2002.

[14] Jacob N. Israelachvili and H. K. Christenson. Liquid structure and short-range forces between surfaces in liquids. Physica A, 140(12):278-284, 1986.

[15] Jacob N. Israelachvili. Measurement of the viscosity of liquids in very thin films. J. Colloid Interface Science, 110(1):263-271, 1986.

[16] Jacob N. Israelachvili and Patricia M. McGuiggan. Forces between surfaces in liquids. Science, 241(4867):795-800, 1988.

[17] Keith E. Gubbins, Ying-Chun Liu, Joshua D. Moore, and Jeremy C. Palmer. The role of molecular modeling in confined systems: impact and prospects. Phys. Chem. Chem. Phys., 13:58-85, 2011.

[18] B. J. Alder and T. E. Wainwright. Studies in Molecular Dynamics. I. General Method. J. Chem. Phys., 31(2):459-466, 1959.

[19] A. Rahman. Correlations in the Motion of Atoms in Liquid Argon. Phys. Rev., 136(2A):A405-A411, 1964.

[20] W. T. Ashurst and W. G. Hoover. Argon shear viscosity via a lennard-jones potential with equilibrium and nonequilibrium molecular dynamics. Phys. Rev. Lett., 31:206-208, 1973.

[21] W. T. Ashurst and W. G. Hoover. Dense-fluid shear viscosity via nonequilibrium molecular dynamics. Phys. Rev. A, 11(2):658-678, 1975.

[22] W. G. Hoover and W. T. Ashurst. Nonequilibrium Molecular Dynamics. Adv. Theo. Chem., 1:1-51, 1975.

[23] D. Levesque, L. Verlet, and Juhani Kürkijarvi. Computer "experiments" on classical fluids. iv. transport properties and time-correlation functions of the lennard-jones liquid near its triple point. Phys. Rev. A, 7(5):1690-1700, 1973.

[24] B. J. Alder, D. M. Gass, and T. E. Wainwright. Studies in molecular dynamics. viii. the transport coefficients for a hard-sphere fluid. J. Chem. Phys., 53(10):3813-3826, 1970. 
[25] William G. Hoover, Anthony J. C. Ladd, Richard B. Hickman, and Brad Lee Holian. Bulk viscosity via nonequilibrium and equilibrium molecular dynamics. Phys. Rev. A, 21:1756-1760, 1980.

[26] Anthony J. C. Ladd. Equations of motion for non-equilibrium molecular dynamics simulations of viscous flow in molecular fluids. Mol. Phys., 53(2):459-463, 1984.

[27] Denis J. Evans and G. P. Morriss. Nonlinear-response theory for steady planar Couette flow. Phys. Rev. A, 30(3):1528-1530, 1984.

[28] Denis J. Evans and G. P. Morriss. Statistical Mechanics of Nonequilibrium Liquids. Cambridge University Press, Cambridge, 2nd edition, 2008.

[29] B. D. Todd and Peter J. Daivis. Nonequilibrium Molecular Dynamics Simulations of Planar Elongational Flow with Spatially and Temporally Periodic Boundary Conditions. Phys. Rev. Lett., 81(5):1118-1121, 1998.

[30] B. D. Todd and Peter J. Daivis. A new algorithm for unrestricted duration nonequilibrium molecular dynamics simulations of planar elongational flow. Comp. Phys. Comm., 117(3):191-199, 1999.

[31] András Baranyai and Peter T. Cummings. Steady state simulation of planar elongation flow by nonequilibrium molecular dynamics. J. Chem. Phys., 110(1):4245, 1999.

[32] Thomas A. Hunt, Stefano Bernardi, and B. D. Todd. A new algorithm for extended nonequilibrium molecular dynamics simulations of mixed flow. J. Chem. Phys., 133(15):154116, 2010.

[33] J. J. Magda, M. Tirrell, and H. T. Davis. Molecular dynamics of narrow, liquidfilled pores. J. Chem. Phys., 83(4):1888-1901, 1985.

[34] I. Bitsanis, J. J. Magda, M. Tirrell, and H. T. Davis. Molecular dynamics of flow in micropores. J. Chem. Phys., 87(3):1733-1750, 1987.

[35] I. Bitsanis, T. K. Vanderlick, M. Tirrell, and H. T. Davis. A tractable molecular theory of flow in strongly inhomogeneous fluids. J. Chem. Phys., 89:3152, 1988.

[36] Ioannis Bitsanis, Susan A. Somers, H. Ted Davis, and Matthew Tirrell. Microscopic dynamics of flow in molecularly narrow pores. J. Chem. Phys., 93(5):34273431,1990 . 
[37] Steven Y. Liem, David Brown, and Julian H. R. Clarke. Investigation of the homogeneous-shear nonequilibrium-molecular-dynamics method. Phys. Rev. A, 45(6):3706-3713, 1992.

[38] Denis J. Evans and Gary P. Morriss. Shear Thickening and Turbulence in Simple Fluids. Phys. Rev. Lett., 56(20):2172-2175, 1986.

[39] Masaharu Isobe. Velocity statistics in two-dimensional granular turbulence. Phys. Rev. E, 68:040301, 2003.

[40] J. A. Cosgrove, J. M. Buick, S. J. Tonge, C. G. Munro, C. A. Greated, and D. M. Campbell. Application of the lattice boltzmann method to transition in oscillatory channel flow. J. Phys. A: Mathematical and General, 36(10):26092620, 2003.

[41] A. P. Markesteijn. Ph.D. Thesis. Delft University of Technology, 2011.

[42] Weinan E, Bjorn Engquist, Xiantao Li, Weiqing Ren, and Eric Vanden-Eijnden. Heterogeneous multiscale methods: A review. Comm. in Comp. Phys., 2(3):367450, 2007.

[43] Denis J. Evans. Computer "experiment" for nonlinear thermodynamics of couette flow. J. Chem. Phys., 78(6):3297-3302, 1983.

[44] István Borzsák, Peter T. Cummings, and Denis J. Evans. Shear viscosity of a simple fluid over a wide range of strain rates. Mol. Phys., 100(16):2735-2738, 2002.

[45] J. P. Ryckaert, A. Bellemans, G. Ciccotti, and G. V. Paolini. Shear-Rate Dependence of the Viscosity of Simple Fluids by Nonequilibrium Molecular Dynamics. Phys. Rev. Lett., 60(2):128-131, 1988.

[46] Hin Hark Gan and Byung Chan Eu. Theory of the nonequilibrium structure of dense simple fluids: Effects of shearing. Phys. Rev. A, 45:3670-3686, 1992.

[47] Rajesh Khare, J. Pablo, and Arun Yethiraj. Molecular simulation and continuum mechanics study of simple fluids in non-isothermal planar Couette flows. $J$. Chem. Phys., 107:2589, 1997.

[48] Susan A. Somers and H. Ted Davis. Microscopic dynamics of fluids confined between smooth and atomically structured solid surfaces. J. Chem. Phys., 96(7):5389-5407, 1992.

[49] Stefano Bernardi, B. D. Todd, and Debra J. Searles. Thermostating highly confined fluids. J. Chem. Phys., 132(24):244706, 2010. 
[50] William G. Hoover and C. G. Hoover. Nonlinear stresses and temperatures in transient adiabatic and shear flows via nonequilibrium molecular dynamics: Three definitions of temperature. Phys. Rev. E, 79:046705, 2009.

[51] Leslie V. Woodcock. Origins of shear dilatancy and shear thickening phenomena. Chem. Phys. Lett., 111(45):455-461, 1984.

[52] Denis J. Evans, Howard J. M. Hanley, and Siegfried Hess. Non-newtonian phenomena in simple fluids. Phys. Today, 37(1):26-33, 1984.

[53] Q. H. Zeng, A. B. Yu, and G. Q. Lu. Multiscale modeling and simulation of polymer nanocomposites. Progress in Polymer Science, 33(2):191-269, 2008.

[54] Alberto Pérez, F. Javier Luque, and Modesto Orozco. Frontiers in molecular dynamics simulations of dna. Accounts of Chemical Research, 45(2):196-205, 2012 .

[55] V. A. Bakaev and W. A. Steele. On the computer simulation of a hydrophobic vitreous silica surface. J. Chem. Phys., 111(21):9803-9812, 1999.

[56] Kazuma Mawatari, Takehiko Tsukahara, and Takehiko Kitamori. Extended nanospace chemical systems on a chip for new analytical technology. Analyst, 136:3051-3059, 2011.

[57] A. P. Markesteijn, Remco Hartkamp, S. Luding, and J. Westerweel. A comparison of the value of viscosity for several water models using poiseuille flow in a nano-channel. J. Chem. Phys., 136(13):134104, 2012.

[58] William G. Hoover. Molecular Dynamics. Lecture Notes in Physics. SpringerVerlag, Heidelberg, 1st edition, 1986.

[59] William G. Hoover. Computational statistical mechanics. Studies in modern thermodynamics. Elsevier, Amsterdam, 1st edition, 1991.

[60] D. Frenkel and B. Smit. Understanding Molecular Simulation. Academic Press, 1st edition, 1996.

[61] M. P. Allen and D. J. Tildesley. Computer Simulation of Liquids. Oxford University Press, Oxford, 1st edition, 1987.

[62] Loup Verlet. Computer "Experiments" on Classical Fluids. I. Thermodynamical Properties of Lennard-Jones Molecules. Phys. Rev., 159(1):98, 1967.

[63] J. P. Hansen and I. R. Mcdonald. The Theory of Simple Liquids. Academic Press, New York, 2nd edition, 1986. 
[64] Song H. Lee and Peter T. Cummings. Effect of three-body forces on the shear viscosity of liquid argon. J. Chem. Phys., 101(7):6206-6209, 1994.

[65] Gianluca Marcelli, B. D. Todd, and Richard J. Sadus. The strain rate dependence of shear viscosity, pressure and energy from two-body and three-body interactions. Fluid Phase Equilibria, 183-184(0):371-379, 2001. Proceedings of the fourteenth symposium on thermophysical properties.

[66] Gianluca Marcelli, B. D. Todd, and Richard J. Sadus. On the relationship between two-body and three-body interactions from nonequilibrium molecular dynamics simulation. J. Chem. Phys., 115(20):9410-9413, 2001.

[67] Gianluca Marcelli, B. D. Todd, and Richard J. Sadus. Erratum: "On the relationship between two-body and three-body interactions from nonequilibrium molecular dynamics simulation" [J. Chem. Phys. 115, 9410 (2001)]. J. Chem. Phys., 120(6):3043-3043, 2004.

[68] J. E. Jones. On the determination of molecular fields. ii. from the equation of state of a gas. Proceedings of the Royal Society of London. Series A, 106(738):463-477, 1924.

[69] Alauddin Ahmed and Richard J. Sadus. Solid-liquid equilibria and triple points of n-6 lennard-jones fluids. J. Chem. Phys., 131(17):174504, 2009.

[70] D. Fincham and D. M. Heyes. Comparisons between experimental argon and lennard-jones 12:6 shear viscosities. Chem. Phys., 78(3):425-441, 1983.

[71] Jean-Pierre Hansen and Loup Verlet. Phase transitions of the lennard-jones system. Phys. Rev., 184(1):151-161, 1969.

[72] Michael F. Pas and Bruno J. Zwolinski. Computation of the transport coefficients of dense fluid neon, argon, krypton and xenon by molecular dynamics. Mol. Phys., 73(3):471-481, 1991.

[73] B. Smit. Phase diagrams of Lennard-Jones fluids. J. Chem. Phys., 96(11):8639$8640,1992$.

[74] Ludovic Berthier and Gilles Tarjus. The role of attractive forces in viscous liquids. J. Chem. Phys., 134(21):214503, 2011.

[75] Yuta Asano and Kazuhiro Fuchizaki. Phase diagram of the modified lennardjones system. J. Chem. Phys., 137(17):174502, 2012.

[76] D. M. Heyes. Transport coefficients of simple fluids with steeply repulsive potentials. J. Phys.: Condensed Matter, 6(32):6409, 1994. 
[77] John D. Weeks, David Chandler, and Hans C. Andersen. Role of Repulsive Forces in Determining the Equilibrium Structure of Simple Liquids. J. Chem. Phys., 54(12):5237-5247, 1971.

[78] Brad Lee Holian and Denis J. Evans. Shear viscosities away from the melting line: A comparison of equilibrium and nonequilibrium molecular dynamics. $J$. Chem. Phys., 78(8):5147-5150, 1983.

[79] Karl P. Travis and Keith E. Gubbins. Poiseuille flow of Lennard-Jones fluids in narrow slit pores. J. Chem. Phys., 112(4):1984-1994, 2000.

[80] John A. White. Lennard-jones as a model for argon and test of extended renormalization group calculations. J. Chem. Phys., 111(20):9352-9356, 1999.

[81] L. A. Rowley, D. Nicholson, and N. G. Parsonage. Monte carlo grand canonical ensemble calculation in a gas-liquid transition region for 12-6 argon. J. Comp. Phys., 17(4):401-414, 1975.

[82] J. A. Barker and D. Henderson. What is "liquid"? Understanding the states of matter. Rev. Mod. Phys., 48:587-671, 1976.

[83] Peter T. Cummings and Denis J. Evans. Nonequilibrium molecular dynamics approaches to transport properties and non-newtonian fluid rheology. Ind. Eng. Chem. Research, 31(5):1237-1252, 1992.

[84] Martin Kröger. Simple models for complex nonequilibrium fluids. Phys. Reports, 390(6):453-551, 2004.

[85] J. H. Irving and John G. Kirkwood. The Statistical Mechanical Theory of Transport Processes. IV. The Equations of Hydrodynamics. J. Chem. Phys., 18(6):817-829, 1950.

[86] B. D. Todd, Denis J. Evans, and Peter J. Daivis. Pressure tensor for inhomogeneous fluids. Phys. Rev. E, 52(2):1627-1638, 1995.

[87] P. Schofield and J. R. Henderson. Statistical Mechanics of Inhomogeneous Fluids. Proceedings of the Royal Society of London. A., 379(1776):231-246, 1982.

[88] David R. J. Monaghan and Gary P. Morriss. Microscopic study of steady convective flow in periodic systems. Phys. Rev. E, 56(1):476-488, 1997.

[89] Isaac Goldhirsch. Stress, stress asymmetry and couple stress: from discrete particles to continuous fields. Granular Matter, 12:239-252, 2010. 
[90] Shengping Shen and S. N. Atluri. Atomic-level Stress Calculation and Continuum-Molecular System Equivalence. Comp. Mod. Eng. Sciences, 6(1):91$104,2004$.

[91] L. Mistura. The definition of the pressure tensor in the statistical mechanics of nonuniform classical fluids. Int. J. Thermophys., 8:397-403, 1987.

[92] A. Harasima. Molecular Theory of Surface Tension. John Wiley \& Sons, Inc., 2007.

[93] D. H. Tsai. The virial theorem and stress calculation in molecular dynamics. J. Chem. Phys., 70(3):1375-1382, 1979.

[94] J. Cormier, J. M. Rickman, and T. J. Delph. Stress calculation in atomistic simulations of perfect and imperfect solids. J. Appl. Phys., 89(1):99-104, 2001.

[95] D. M. Heyes, E. R. Smith, D. Dini, and T. A. Zaki. The equivalence between volume averaging and method of planes definitions of the pressure tensor at a plane. J. Chem. Phys., 135(2):024512, 2011.

[96] Jacob Sonne, Flemming Y. Hansen, and Gunther H. Peters. Methodological problems in pressure profile calculations for lipid bilayers. J. Chem. Phys., 122(12):124903, 2005.

[97] F. Varnik, J. Baschnagel, and K. Binder. Molecular dynamics results on the pressure tensor of polymer films. J. Chem. Phys., 113(10):4444-4453, 2000.

[98] Björn Hafskjold and Tamio Ikeshoji. Microscopic pressure tensor for hard-sphere fluids. Phys. Rev. E, 66:011203, 2002.

[99] John M. Dealy. Official nomenclature for material functions describing the response of a viscoelastic fluid to various shearing and extensional deformations. J. Rheol., 28(3):181-195, 1984.

[100] L. E. Malvern. Introduction to the Mechanics of a Continuous Medium. Prentice Hall, Englewood Cliffs, New Jersey, 1969.

[101] Denis J. Evans. Molecular dynamics simulations of the rheological properties of simple fluids. Phys. A, 118(1-3):51-68, 1983.

[102] Peter J. Daivis, Benjamin A. Dalton, and Tetsuya Morishita. Effect of kinetic and configurational thermostats on calculations of the first normal stress coefficient in nonequilibrium molecular dynamics simulations. Phys. Rev. E, 86:056707, 2012 . 
[103] Karl P. Travis, Peter J. Daivis, and Denis J. Evans. Thermostats for molecular fluids undergoing shear flow: Application to liquid chlorine. J. Chem. Phys., 103(24):10638-10651, 1995.

[104] Xin Yong and Lucy T. Zhang. Nanoscale simple-fluid behavior under steady shear. Phys. Rev. E, 85:051202, 2012.

[105] Gary P. Morriss and Lamberto Rondoni. Definition of temperature in equilibrium and nonequilibrium systems. Phys. Rev. E, 59:R5-R8, 1999.

[106] Jerome Delhommelle, J. Petravic, and Denis J. Evans. On the effects of assuming flow profiles in nonequilibrium simulations. J. Chem. Phys., 119(21):1100511010, 2003.

[107] Jerome J. Erpenbeck. Shear viscosity of the hard-sphere fluid via nonequilibrium molecular dynamics. Phys. Rev. Lett., 52(15):1333-1335, 1984.

[108] Denis J. Evans, S. T. Cui, H. J. M. Hanley, and G. C. Straty. Conditions for the existence of a reentrant solid phase in a sheared atomic fluid. Phys. Rev. A, 46:6731-6734, 1992.

[109] H. J. C. Berendsen, J. P. M. Postma, W. F. van Gunsteren, A. DiNola, and J. R. Haak. Molecular dynamics with coupling to an external bath. J. Chem. Phys., 81(8):3684-3690, 1984.

[110] S. A. Adelman and J. D. Doll. Generalized langevin equation approach for atom/solid? surface scattering: General formulation for classical scattering off harmonic solids. J. Chem. Phys., 64(6):2375-2388, 1976.

[111] Thomas Soddemann, Burkhard Dünweg, and Kurt Kremer. Dissipative particle dynamics: A useful thermostat for equilibrium and nonequilibrium molecular dynamics simulations. Phys. Rev. E, 68:046702, 2003.

[112] L. V. Woodcock. Isothermal molecular dynamics calculations for liquid salts. Chem. Phys. Lett., 10(3), 1971.

[113] William G. Hoover, Anthony J. C. Ladd, and Bill Moran. High-strain-rate plastic flow studied via nonequilibrium molecular dynamics. Phys. Rev. Lett., 48:18181820,1982 .

[114] Denis J. Evans, William G. Hoover, Bruce H. Failor, Bill Moran, and Anthony J. C. Ladd. Nonequilibrium molecular dynamics via gauss's principle of least constraint. Phys. Rev. A, 28:1016-1021, 1983. 
[115] William G. Hoover. Adiabatic Hamiltonian deformation, linear response theory, and nonequilibrium molecular dynamics. In Luis Garrido, editor, Systems Far from Equilibrium, volume 132 of Lecture Notes in Physics, pages 373-380. Springer Berlin / Heidelberg, 1st edition, 1980.

[116] C. P. Dettmann and G. P. Morriss. Hamiltonian formulation of the gaussian isokinetic thermostat. Phys. Rev. E, 54:2495-2500, 1996.

[117] B. D. Todd and Peter J. Daivis. Homogeneous non-equilibrium molecular dynamics simulations of viscous flow: techniques and applications. Mol. Simul., 33:189-229, 2007.

[118] Shuichi Nosé. A molecular dynamics method for simulations in the canonical ensemble. Mol. Phys., 52(2):255-268, 1984.

[119] W. G. Hoover and C. G. Hoover. Links between microscopic and macroscopic fluid mechanics. Mol. Phys., 101(11):1559-1573, 2003.

[120] Shuichi Nosé. A unified formulation of the constant temperature molecular dynamics methods. J. Chem. Phys., 81(1):511-519, 1984.

[121] William G. Hoover. Canonical dynamics: Equilibrium phase-space distributions. Phys. Rev. A, 31(3):1695-1697, 1985.

[122] Glenn J. Martyna, Michael L. Klein, and Mark Tuckerman. Nosé-Hoover chains: The canonical ensemble via continuous dynamics. J. Chem. Phys., 97(4):26352643,1992 .

[123] Stephen D. Bond, Benedict J. Leimkuhler, and Brian B. Laird. The NoséPoincaré method for constant temperature molecular dynamics. J. Comp. Phys., 151(1):114-134, 1999.

[124] B. D. Todd and Peter J. Daivis. The stability of nonequilibrium molecular dynamics simulations of elongational flows. J. Chem. Phys., 112(1):40-46, 2000.

[125] Hans Henrik Rugh. A geometric, dynamical approach to thermodynamics. J. Phys. A, 31(38):7761, 1998.

[126] L. Lue and Denis J. Evans. Configurational temperature for systems with constraints. Phys. Rev. E, 62:4764-4768, 2000.

[127] Jerome Delhommelle and Denis J. Evans. Configurational temperature thermostat for fluids undergoing shear flow: application to liquid chlorine. Mol. Phys., 99(21):1825-1829, 2001. 
[128] Jerome Delhommelle, J. Petravic, and Denis J. Evans. Reexamination of string phase and shear thickening in simple fluids. Phys. Rev. E, 68(3):031201, 2003.

[129] Carlos Braga and Karl P. Travis. A configurational temperature Nosé-Hoover thermostat. J. Chem. Phys., 123(13):134101, 2005.

[130] Carlos Braga and Karl P. Travis. Configurational constant pressure molecular dynamics. J. Chem. Phys., 124(10):104102, 2006.

[131] Karl P. Travis and Carlos Braga. Configurational temperature control for atomic and molecular systems. J. Chem. Phys., 128(1):014111, 2008.

[132] P. P. Ewald. Die Berechnung optischer und elektrostatischer Gitterpotentiale. Annalen der Physik, 369(3):253-287, 1921.

[133] D. Wolf, P. Keblinski, S. R. Phillpot, and J. Eggebrecht. Exact method for the simulation of coulombic systems by spherically truncated, pairwise $r^{-1}$ summation. J. Chem. Phys., 110(17):8254-8282, 1999.

[134] D. J. Adams. On the use of the ewald summation in computer simulation. J. Chem. Phys., 78(5):2585-2590, 1983.

[135] Eleftherios E. Gdoutos, Ravi Agrawal, and Horacio D. Espinosa. Comparison of the ewald and wolf methods for modeling electrostatic interactions in nanowires. International Journal for Numerical Methods in Engineering, 84(13):1541-1551, 2010.

[136] Aidan P. Thompson, Steven J. Plimpton, and William Mattson. General formulation of pressure and stress tensor for arbitrary many-body interaction potentials under periodic boundary conditions. J. Chem. Phys., 131(15):154107, 2009 .

[137] In-Chul Yeh and Gerhard Hummer. System-size dependence of diffusion coefficients and viscosities from molecular dynamics simulations with periodic boundary conditions. J. Phys. Chem. B, 108(40):15873-15879, 2004.

[138] M. Schoen and C. Hoheisel. The shear viscosity of a lennard-jones fluid calculated by equilibrium molecular dynamics. Mol. Phys., 56(3):653-672, 1985.

[139] Karsten Meier, Arno Laesecke, and Stephan Kabelac. Transport coefficients of the lennard-jones model fluid. i. viscosity. J. Chem. Phys., 121(8):3671-3687, 2004 .

[140] Lars Onsager. Reciprocal relations in irreversible processes. i. Phys. Rev., 37(4):405-426, 1931. 
[141] Lars Onsager. Reciprocal relations in irreversible processes. ii. Phys. Rev., 38(12):2265-2279, 1931.

[142] B. L. Holian and Denis J. Evans. Classical response theory in the Heisenberg picture. J. Chem. Phys., 83(7):3560-3566, 1985.

[143] B. D. Todd. Nonlinear response theory for time-periodic elongational flows. Phys. Rev. E, 58(4):4587-4593, 1998.

[144] G. P. Morriss and Denis J. Evans. Isothermal response theory. Mol. Phys., 54(3):629-636, 1985.

[145] Peter J. Daivis and B. D. Todd. A simple, direct derivation and proof of the validity of the SLLOD equations of motion for generalized homogeneous flows. J. Chem. Phys., 124:194103, 2006.

[146] William G. Hoover. Liouville's theorems, Gibbs' entropy, and multifractal distributions for nonequilibrium steady states. J. Chem. Phys., 109(11):4164-4170, 1998.

[147] Thomas Weinhart, Remco Hartkamp, Anthony R. Thornton, and Stefan Luding. 3D coarse grained continuum description of granular flow down an inclined surface. submitted, 2013.

[148] William G. Hoover, Carol G. Hoover, and Janka Petravic. Simulation of two- and three-dimensional dense-fluid shear flows via nonequilibrium molecular dynamics: Comparison of time-and-space-averaged stresses from homogeneous Doll's and Sllod shear algorithms with those from boundary-driven shear. Phys. Rev. E, 78(4):046701, 2008.

[149] Roger Edberg, G. P. Morriss, and Denis J. Evans. Rheology of n-alkanes by nonequilibrium molecular dynamics. J. Chem. Phys., 86(8):4555-4570, 1987.

[150] Karl P. Travis, Peter J. Daivis, and Denis J. Evans. Computer simulation algorithms for molecules undergoing planar couette flow: A nonequilibrium molecular dynamics study. J. Chem. Phys., 103(3):1109-1118, 1995.

[151] Karl P. Travis, Peter J. Daivis, and Denis J. Evans. Erratum: Thermostats for molecular fluids undergoing shear flow: Application to liquid chlorine. J. Chem. Phys., 105(9):3893-3894, 1996.

[152] D. M. Heyes. Molecular dynamics simulations of extensional, sheet and unidirectional flow. Chem. Phys., 98(1), 1985. 
[153] A. M. Kraynik and D. A. Reinelt. Extensional motions of spatially periodic lattices. Int. J. Multiphase Flow, 18(6), 1992.

[154] Denis J. Evans and G. P. Morriss. Non-equilibrium molecular-dynamics simulation of Couette flow in two-dimensional fluids. Phys. Rev. Lett., 51(19):1776, 1983.

[155] Thomas A. Hunt and B. D. Todd. Diffusion of linear polymer melts in shear and extensional flows. J. Chem. Phys., 131(5):054904, 2009.

[156] M. W. Evans and D. M. Heyes. Combined shear and elongational flow by nonequilibrium molecular dynamics. Mol. Phys., 69(2):241-263, 1990.

[157] Federico Frascoli and B. D. Todd. Molecular dynamics simulation of planar elongational flow at constant pressure and constant temperature. J. Chem. Phys., 126(4):044506, 2007.

[158] Joo Sung Lee, Eric S. G. Shaqfeh, and Susan J. Muller. Dynamics of dna tumbling in shear to rotational mixed flows: Pathways and periods. Phys. Rev. E, 75:040802, 2007.

[159] Joe S. Hur, Eric S. G. Shaqfeh, Hazen P. Babcock, and Steven Chu. Dynamics and configurational fluctuations of single dna molecules in linear mixed flows. Phys. Rev. E, 66:011915, 2002.

[160] Eric S. G. Shaqfeh. The dynamics of single-molecule DNA in flow. J. NonNewtonian Fluid Mech., 130(1):1-28, 2005.

[161] Brendan D. Hoffman and Eric S. G. Shaqfeh. The dynamics of the coil-stretch transition for long, flexible polymers in planar mixed flows. J. Rheol., 51(5):947969, 2007.

[162] N. J. Woo and Eric S. G. Shaqfeh. The configurational phase transitions of flexible polymers in planar mixed flows near simple shear. J. Chem. Phys., 119(5):2908-2914, 2003.

[163] Charles E. Sing and Alfredo Alexander-Katz. Dynamics of collapsed polymers under the simultaneous influence of elongational and shear flows. J. Chem. Phys., 135(1):014902, 2011.

[164] Joo Sung Lee, Rebecca Dylla-Spears, Nerayo P. Teclemariam, and Susan J. Muller. Microfluidic four-roll mill for all flow types. Appl. Phys. Lett., 90(7):074103, 2007. 
[165] Denis J. Evans, E. G. D. Cohen, and Gary P. Morriss. Viscosity of a simple fluid from its maximal Lyapunov exponents. Phys. Rev. A, 42(10):5990-5997, 1990.

[166] Federico Frascoli, Debra J. Searles, and B. D. Todd. Chaotic properties of planar elongational flow and planar shear flow: Lyapunov exponents, conjugate-pairing rule, and phase space contraction. Phys. Rev. E, 73(4):046206, 2006.

[167] Federico Frascoli, Debra J. Searles, and B. D. Todd. Chaotic properties of isokinetic-isobaric atomic systems under planar shear and elongational flows. Phys. Rev. E, 77(5):056217, 2008.

[168] Grant S. Heffelfinger and Frank van Swol. Diffusion in Lennard-Jones fluids using dual control volume grand canonical molecular dynamics simulation (DCVGCMD). J. Chem. Phys., 100(10):7548-7552, 1994.

[169] H. A. Posch and W. G. Hoover. Lyapunov instability of dense Lennard-Jones fluids. Phys. Rev. A, 38(1):473-482, 1988.

[170] Massimo Falcioni, Stefano Isola, and Angelo Vulpiani. Correlation functions and relaxation properties in chaotic dynamics and statistical mechanics. Phys. Lett. A, 144(6-7):341-346, 1990.

[171] Robert Zwanzig. Time-correlation functions and transport coefficients in statistical mechanics. Ann. Rev. Phys. Chem., 16(1):67-102, 1965.

[172] B. J. Alder and T. E. Wainwright. Decay of the velocity autocorrelation function. Phys. Rev. A, 1:18-21, 1970.

[173] R. F. A. Dib, F. Ould-Kaddour, and D. Levesque. Long-time behavior of the velocity autocorrelation function at low densities and near the critical point of simple fluids. Phys. Rev. E, 74:011202, 2006.

[174] Stephen R. Williams, G. Bryant, I. K. Snook, and W. van Megen. Velocity autocorrelation functions of hard-sphere fluids: Long-time tails upon undercooling. Phys. Rev. Lett., 96:087801, 2006.

[175] Masaharu Isobe. Long-time tail of the velocity autocorrelation function in a twodimensional moderately dense hard-disk fluid. Phys. Rev. E, 77:021201, 2008.

[176] E. G. D. Cohen. Kinetic theory of non-equilibrium fluids. Physica A, 118(13):17-42, 1983.

[177] M. H. Ernst, E. H. Hauge, and J. M. J. van Leeuwen. Asymptotic time behavior of correlation functions. ii. kinetic and potential terms. J. Stat. Phys., 15:7-22, 1976 . 
[178] Jerome J. Erpenbeck and William W. Wood. Molecular dynamics calculations of shear viscosity time-correlation functions for hard spheres. J. Stat. Phys., 24:455-468, 1981.

[179] T. R. Kirkpatrick. Large long-time tails and shear waves in dense classical liquids. Phys. Rev. Lett., 53:1735-1738, 1984.

[180] T. R. Kirkpatrick and J. C. Nieuwoudt. Mode-coupling theory of the large longtime tails in the stress-tensor autocorrelation function. Phys. Rev. A, 33:26512657,1986 .

[181] I. M. de Schepper, A. F. E. M. Haffmans, and H. van Beijeren. Comment on "Large long-time tails and shear waves in dense classical liquids". Phys. Rev. Lett., 56:538-538, 1986.

[182] Anthony J. C. Ladd, W. Edward Alley, and Berni J. Alder. Structural relaxation in dense hard-sphere fluids. J. Stat. Phys., 48:1147-1156, 1987.

[183] M. Isobe and B. J. Alder. Molasses tail in two dimensions. Mol. Phys., 107(4):609-613, 2009.

[184] R. M. Puscasu, B. D. Todd, Peter J. Daivis, and J. S. Hansen. Nonlocal viscosity of polymer melts approaching their glassy state. J. Chem. Phys., 133(14):144907, 2010 .

[185] Peter J. Daivis. Thermodynamic relationships for shearing linear viscoelastic fluids. J. Non-Newtonian Fluid Mech., 152:120-128, 2008.

[186] Melville S. Green. Markoff Random Processes and the Statistical Mechanics of Time-Dependent Phenomena. II. Irreversible Processes in Fluids. J. Chem. Phys., 22(3):398-413, 1954.

[187] Ryogo Kubo. Statistical-Mechanical Theory of Irreversible Processes. I. General Theory and Simple Applications to Magnetic and Conduction Problems. Journal of the Physical Society of Japan, 12(6):570-586, 1957.

[188] Karl P. Travis, Debra J. Searles, and Denis J. Evans. Strain rate dependent properties of a simple fluid. Mol. Phys., 95(2):195-202, 1998.

[189] James W. Dufty and Michael J. Lindenfeld. Nonlinear transport in the Boltzmann limit. J. Stat. Phys., 20:259-301, 1979.

[190] Caroline Desgranges and Jerome Delhommelle. Molecular simulation of transport in nanopores: Application of the transient-time correlation function formalism. Phys. Rev. E, 77(2):027701, 2008. 
[191] Stefano Bernardi, Sarah J. Brookes, Debra J. Searles, and Denis J. Evans. Response theory for confined systems. J. Chem. Phys., 137(7):074114, 2012.

[192] Robert Zwanzig and Raymond D. Mountain. High-frequency elastic moduli of simple fluids. J. Chem. Phys., 43(12):4464-4471, 1965.

[193] H. J. M. Hanley, J. C. Rainwater, and S. Hess. Shear-induced angular dependence of the liquid pair correlation function. Phys. Rev. A, 36:1795-1802, 1987.

[194] B. D. Todd and Peter J. Daivis. Nonequilibrium Molecular Dynamics: Theory, Algorithms and Applications. Cambridge University Press, Cambridge, 1st edition, 2013.

[195] Yu. V. Kalyuzhnyi, S. T. Cui, Peter T. Cummings, and H. D. Cochran. Distribution functions of a simple fluid under shear: Low shear rates. Phys. Rev. E, 60:1716-1723, 1999.

[196] Yu. V. Kalyuzhnyi, S. T. Cui, and H. D. Cochran. Distribution functions of a simple fluid under shear. ii. high shear rates. Phys. Rev. E, 63:011209, 2000.

[197] Noel A. Clark and Bruce J. Ackerson. Observation of the coupling of concentration fluctuations to steady-state shear flow. Phys. Rev. Lett., 44:1005-1008, 1980.

[198] Siegfried Hess. Shear-flow-induced distortion of the pair-correlation function. Phys. Rev. A, 22:2844-2848, 1980.

[199] Siegfried Hess. Shear-flow induced distortion of the radial distribution function, test of a model kinetic equation in the nonlinear flow regime. Physica A, 118:444, 1983.

[200] Siegfried Hess. Similarities and differences in the nonlinear flow behavior of simple and of molecular liquids. Physica A, 118:79-104, 1983.

[201] Siegfried Hess and H. J. M. Hanley. Pressure tensor and viscosity coefficients of a soft sphere liquid under shear. Int. J. Thermophys., 4:97-114, 1983.

[202] J. F. Schwarzl and S. Hess. Shear-flow-induced distortion of the structure of a fluid: Application of a simple kinetic equation. Phys. Rev. A, 33:4277-4283, 1986 .

[203] Hin Hark Gan and Byung Chan Eu. Theory of the nonequilibrium structure of dense simple fluids. ii. high-shear-rate effects. Phys. Rev. A, 46:6344-6358, 1992. 
[204] Hikmat Farhat and Byung Chan Eu. Monte carlo method and the nonequilibrium structure and non-newtonian viscosity of a sheared simple liquid. J. Chem. Phys., 110(1):97-108, 1999.

[205] V. Nosenko, A. V. Ivlev, and G. E. Morfill. Microstructure of a liquid twodimensional dusty plasma under shear. Phys. Rev. Lett., 108:135005, 2012.

[206] J. Liam McWhirter and G. N. Patey. Nonequilibrium molecular dynamics simulations of a simple dipolar fluid under shear flow. J. Chem. Phys., 117(6):27472761, 2002.

[207] M. N. Hounkonnou, C. Pierleoni, and J. P. Ryckaert. Liquid chlorine in shear and elongational flows: A nonequilibrium molecular dynamics study. J. Chem. Phys., 97(12):9335-9344, 1992.

[208] M. Alam and S. Luding. First normal stress difference and crystallization in a dense sheared granular fluid. Phys. Fluids, 15(8):2298-2312, 2003.

[209] R. F. Berg, M. R. Moldover, and G. A. Zimmerli. Viscoelasticity of xenon near the critical point. Phys. Rev. Lett., 82:920-923, 1999.

[210] R. F. Berg. Fluids near a critical point obey a generalized Cox-Merz rule. J. Rheol., 48(6):1365-1373, 2004.

[211] J. G. Powles and D. M. Heyes. Viscoelastic behaviour of fluids with steeply repulsive potentials. Mol. Phys., 98(14):917-927, 2000.

[212] A. C. Brańka and D. M. Heyes. Time correlation functions of hard sphere and soft sphere fluids. Phys. Rev. E, 69:021202, 2004.

[213] Jung Gun Nam, Kyung Hyun Ahn, Seung Jong Lee, and Kyu Hyun. First normal stress difference of entangled polymer solutions in large amplitude oscillatory shear flow. J. Rheol., 54(6):1243-1266, 2010.

[214] Huan-Chang Tseng, Jiann-Shing Wu, and Rong-Yeu Chang. Linear viscoelasticity and thermorheological simplicity of n-hexadecane fluids under oscillatory shear via non-equilibrium molecular dynamics simulations. Phys. Chem. Chem. Phys., 12:4051-4065, 2010.

[215] R. Byron Bird, R. C. Armstrong, and Ole Hassager. Dynamics of Polymeric Liquids, Fluid Mechanics (Dynamics of Polymer Liquids), volume 1. WileyInterscience, New York, 2nd edition, 1987.

[216] Bernard D. Coleman and Hershel Markovitz. Normal stress effects in secondorder fluids. J. Appl. Phys., 35(1):1-9, 1964. 
[217] Peter J. Daivis and Denis J. Evans. Comparison of constant pressure and constant volume nonequilibrium simulations of sheared model decane. J. Chem. Phys., 100(1):541-547, 1994.

[218] U. Balucani, R. Vallauri, and T. Gaskell. Stress autocorrelation function and structural relaxation in liquid rubidium. Phys. Scripta, T25:290, 1989.

[219] Stephen R. Williams and Denis J. Evans. Viscoelastic properties of crystals. J. Chem. Phys., 131(2):024115, 2009.

[220] Stephen R. Williams and Denis J. Evans. Erratum: "Viscoelastic properties of crystals" [J. Chem. Phys. 131, 024115 (2009)]. J. Chem. Phys., 131(15):159902, 2009 .

[221] D. M. Heyes. Transport coefficients of lennard-jones fluids: A moleculardynamics and effective-hard-sphere treatment. Phys. Rev. B, 37:5677-5696, 1988.

[222] Siegfried Hess and Denis J. Evans. Computation of the viscosity of a liquid from time averages of stress fluctuations. Phys. Rev. E, 64:011207, 2001.

[223] J. Clerk Maxwell. On the dynamical theory of gases. Philosophical Transactions of the Royal Society of London, 157:49-88, 1867.

[224] R. C. Picu and J. H. Weiner. Structural changes during stress relaxation in simple liquids. J. Chem. Phys., 107(18):7214-7222, 1997.

[225] Raymond D. Mountain and Robert Zwanzig. Shear relaxation times of simple fluids. J. Chem. Phys., 44(7):2777-2779, 1966.

[226] Raymond D. Mountain. Relaxation times for the volume viscosity of simple fluids. J. Chem. Phys., 48(5):2189-2190, 1968.

[227] E. Keshavarzi, M. Vahedpour, S. Alavi, and B. Najafi. High-frequency shear modulus and relaxation time of soft-sphere and lennardjones fluids. Int. J. Thermophys., 25:1747-1762, 2004.

[228] G. A. Nunez, G. S. Ribeiro, M. S. Arney, J. Feng, and D. D. Joseph. Rod climbing and normal stresses in heavy crude oils at low shears. J. Rheol., 38(5):1251-1270, 1994.

[229] W. Gleile and N. Ohl. On the relaxation of shear and normal stresses of viscoelastic fluids following constant shear rate experiments. Rheol. Acta, 29:261-280, 1990 . 
[230] Werner Loose and Siegfried Hess. Nonequilibrium velocity distribution function of gases: Kinetic theory and molecular dynamics. Phys. Rev. A, 37:2099-2111, 1988.

[231] V. Garzó and A. Santos. Kinetic Theory of Gases in Shear Flows: Nonlinear Transport. Fundamental Theories of Physics. Kluwer Academic Publishers, Dordrecht, 2003.

[232] Karsten Meier, Arno Laesecke, and Stephan Kabelac. Transport coefficients of the lennard-jones model fluid. ii self-diffusion. J. Chem. Phys., 121(19):9526$9535,2004$.

[233] Fabrcio Da Costa Silva, Luiz A. F. Coelho, Frederico W. Tavares, and Mrcio J. E. M. Cardoso. Shear viscosity calculated by perturbation theory and molecular dynamics for dense fluids. Int. J. Quantum Chem., 95(2):79-87, 2003.

[234] Vladimir G. Baidakov, Sergey P. Protsenko, and Zaliya R. Kozlova. Metastable Lennard-Jones fluids. I. Shear viscosity. J. Chem. Phys., 137(16):164507, 2012.

[235] B. D. Todd. Power-law exponents for the shear viscosity of non-newtonian simple fluids. Phys. Rev. E, 72:041204, 2005.

[236] M. Cappelezzo, C. A. Capellari, S. H. Pezzin, and L. A. F. Coelho. Stokeseinstein relation for pure simple fluids. J. Chem. Phys., 126(22):224516, 2007.

[237] R. Rowley and M. Painter. Diffusion and viscosity equations of state for a lennard-jones fluid obtained from molecular dynamics simulations. Int. J. Thermophys., 18:1109-1121, 1997.

[238] C. Truesdell and R.G. Muncaster. Fundamentals of Maxwell's Kinetic Theory of a Simple Monatomic Gas: Treated As a Branch of Rational Mechanics. Number v. 83 in Pure and Applied Mathematics. Academic Press, 1980.

[239] Niels Boye Olsen, Jeppe C. Dyre, and Tage Christensen. Structural relaxation monitored by instantaneous shear modulus. Phys. Rev. Lett., 81:1031-1033, 1998.

[240] Remco Hartkamp, A. Ghosh, T. Weinhart, and S. Luding. A study of the anisotropy of stress in a fluid confined in a nanochannel. J. Chem. Phys., 137(4):044711, 2012.

[241] P. K. Kundu, I. M. Cohen, and D. R. Dowling. Fluid Mechanics. Elsevier Science, Amsterdam, 5th edition, 2011. 
[242] C. Thornton and L. Zhang. A numerical examination of shear banding and simple shear non-coaxial flow rules. Philosophical Magazine, 86(21-22):3425-3452, 2006.

[243] Jin Sun and Sankaran Sundaresan. A constitutive model with microstructure evolution for flow of rate-independent granular materials. J. Fluid Mech., 682:590-616, 2011.

[244] C. Thornton and L. Zhang. On the evolution of stress and microstructure during general 3D deviatoric straining of granular media. Géotechnique, 60(5):333-341, 2010 .

[245] Hershel Markovitz and Bernard D. Coleman. Nonsteady helical flows of secondorder fluids. Phys. Fluids, 7(6):833-841, 1964.

[246] Bernard D. Coleman and Walter Noll. An approximation theorem for functionals, with applications in continuum mechanics. Archive for Rational Mechanics and Analysis, 6:355-370, 1960.

[247] Peter J. Daivis, M. L. Matin, and B. D. Todd. Nonlinear shear and elongational rheology of model polymer melts at low strain rates. J. Non-Newtonian Fluid Mech., 147(1-2):35-44, 2007.

[248] M. Slemrod. Constitutive relations for rivlin?ericksen fluids based on generalized rational approximation. Archive for Rational Mechanics and Analysis, 146:7393, 1999.

[249] H. Bruus. Theoretical Microfluidics. Oxford Master Series in Condensed Matter Physics. Oxford University Press, New York, 2007.

[250] James T. Jenkins. Dense inclined flows of inelastic spheres. Granular Matter, 10:47-52, 2007.

[251] Jialin Ge, Gianluca Marcelli, B. D. Todd, and Richard J. Sadus. Energy and pressure of shearing fluids at different state points. Phys. Rev. E, 64:021201, 2001.

[252] Denis J. Evans and Gary P. Morriss. Asymptotic nonlinear stress tensor in small periodic systems undergoing couette flow. Phys. Rev. A, 36:4119-4122, 1987.

[253] S. Luding. Structure and cluster formation in granular media. Pramana-J. Phys., 64(6):893-902, 2005.

[254] Remco Hartkamp, Stefano Bernardi, and B. D. Todd. Transient-time correlation function applied to mixed shear and elongational flows. J. Chem. Phys., 136(6):064105, 2012. 
[255] R. C. Picu and J. H. Weiner. Stress relaxation in a diatomic liquid. J. Chem. Phys., 108(12):4984-4991, 1998.

[256] D. M. Heyes, J. J. Kim, C. J. Montrose, and T. A. Litovitz. Time dependent nonlinear shear stress effects in simple liquids: A molecular dynamics study. $J$. Chem. Phys., 73(8):3987-3996, 1980.

[257] J. Gao and J. H. Weiner. Computer simulation of viscoelasticity in polymer melts. Macromol., 25(4):1348-1356, 1992.

[258] P. Attané, J. M. Pierrard, and G. Turrel. Steady and transient shear flows of polystyrene solutions i: Concentration and molecular weight dependence of nondimensional viscometric functions. J. Non-Newtonian Fluid Mech., 18(3):295$317,1985$.

[259] Vagelis A. Harmandaris, Vlasis G. Mavrantzas, and Doros N. Theodorou. Atomistic molecular dynamics simulation of stress relaxation upon cessation of steadystate uniaxial elongational flow. Macromol., 33(21):8062-8076, 2000.

[260] J. T. Padding and W. J. Briels. Coarse-grained molecular dynamics simulations of polymer melts in transient and steady shear flow. J. Chem. Phys., 118(22):10276-10286, 2003.

[261] J. T. Padding and W. J. Briels. Time and length scales of polymer melts studied by coarse-grained molecular dynamics simulations. J. Chem. Phys., 117(2):925$943,2002$.

[262] J. D. Moore, S. T. Cui, H. D. Cochran, and Peter T. Cummings. Transient rheology of a polyethylene melt under shear. Phys. Rev. E, 60:6956-6959, 1999.

[263] Ji-Xuan Hou, Carsten Svaneborg, Ralf Everaers, and Gary S. Grest. Stress relaxation in entangled polymer melts. Phys. Rev. Lett., 105:068301, 2010.

[264] D. Pearson, E. Herbolzheimer, N. Grizzuti, and G. Marrucci. Transient behavior of entangled polymers at high shear rates. J. Polymer Science Part B: Polymer Physics, 29(13):1589-1597, 1991.

[265] C. F. Curtiss and R. Byron Bird. A kinetic theory for polymer melts. ii. the stress tensor and the rheological equation of state. J. Chem. Phys., 74(3):2026-2033, 1981.

[266] R. Byron Bird, H. H. Saab, and C. F. Curtiss. A kinetic theory for polymer melts. 3. elongational flows. J. Phys. Chem., 86(7):1102-1106, 1982. 
[267] H. H. Saab, R. Byron Bird, and C. F. Curtiss. A kinetic theory for polymer melts. v. experimental comparisons for shear-flow rheological properties. J. Chem. Phys., 77(9):4758-4766, 1982.

[268] R. Byron Bird, H. H. Saab, and C. F. Curtiss. A kinetic theory for polymer melts. iv. rheological properties for shear flows. J. Chem. Phys., 77(9):4747-4757, 1982.

[269] Robert A. Stratton and Allan F. Butcher. Stress relaxation upon cessation of steady flow and the overshoot effect of polymer solutions. J. Polymer Science: Polymer Physics Edition, 11(9):1747-1758, 1973.

[270] Chi C. Hua, Jay D. Schieber, and D. C. Venerus. Segment connectivity, chainlength breathing, segmental stretch, and constraint release in reptation models. iii. shear flows. J. Rheol., 43(3):701-717, 1999.

[271] J. W. Rudisill and Peter T. Cummings. The contribution of internal degrees of freedom to the non-newtonian rheology of model polymer fluids. Rheol. Acta, 30:33-43, 1991.

[272] J. W. Rudisill and Peter T. Cummings. Non-equilibrium molecular dynamics approach to the rheology of model polymer fluids. Fluid Phase Equilibria, 88(0):99-113, 1993.

[273] Scott Bair, Clare McCabe, and Peter T. Cummings. Comparison of nonequilibrium molecular dynamics with experimental measurements in the nonlinear shear-thinning regime. Phys. Rev. Lett., 88:058302, 2002.

[274] G. Ciccotti and G. Jacucci. Direct computation of dynamical response by molecular dynamics: The mobility of a charged lennard-jones particle. Phys. Rev. Lett., 35:789-792, 1975.

[275] G. Ciccotti, G. Jacucci, and I. R. McDonald. Transport properties of molten alkali halides. Phys. Rev. A, 13:426-436, 1976.

[276] G. Ciccotti, G. Jacucci, and I.R. McDonald. "Thought-experiments" by molecular dynamics. J. Stat. Phys., 21:1-22, 1979.

[277] Denis J. Evans and Gary P. Morriss. Transient-time-correlation functions and the rheology of fluids. Phys. Rev. A, 38(8):4142-4148, 1988.

[278] Gary P. Morriss and Denis J. Evans. Application of transient correlation functions to shear flow far from equilibrium. Phys. Rev. A, 35(2):792-797, 1987. 
[279] Caroline Desgranges and Jerome Delhommelle. Shear viscosity of liquid copper at experimentally accessible shear rates: Application of the transient-time correlation function formalism. J. Chem. Phys., 128(8):084506, 2008.

[280] B. D. Todd. Application of transient-time correlation functions to nonequilibrium molecular-dynamics simulations of elongational flow. Phys. Rev. E, 56(6):6723$6728,1997$.

[281] Guoai Pan and Clare McCabe. Prediction of viscosity for molecular fluids at experimentally accessible shear rates using the transient time correlation function formalism. J. Chem. Phys., 125(19):194527, 2006.

[282] Oleg A. Mazyar, Guoai Pan, and Clare McCabe. Transient time correlation function calculation of the viscosity of a molecular fluid at low shear rates: a comparison of stress tensors. Mol. Phys., 107(14):1423-1429, 2009.

[283] Denis J. Evans and G. P. Morriss. Equilibrium time correlation functions under gaussian isothermal dynamics. Chem. Phys., 87(3), 1984.

[284] Caroline Desgranges and Jerome Delhommelle. Accurate determination of normal stress differences via transient-time correlation function-non-equilibrium molecular dynamics (TTCF-NEMD) simulations. Mol. Simul., 35(5):405-408, 2009 .

[285] Peter J. Daivis, M. L. Matin, and B. D. Todd. Nonlinear shear and elongational rheology of model polymer melts by non-equilibrium molecular dynamics. $J$. Non-Newtonian Fluid Mech., 111(1):1-18, 2003.

[286] Kurt Kremer and Gary S. Grest. Dynamics of entangled linear polymer melts: A molecular-dynamics simulation. J. Chem. Phys., 92(8):5057-5086, 1990.

[287] Thomas A. Hunt and B. D. Todd. A comparison of model linear chain molecules with constrained and flexible bond lengths under planar couette and extensional flows. Mol. Simul., 35(14):1153-1167, 2009.

[288] D. S. Pearson, G. Ver Strate, E. Von Meerwall, and F. C. Schilling. Viscosity and self-diffusion coefficient of linear polyethylene. Macromol., 20(5):1133-1141, 1987.

[289] Maurizio Mondello and Gary S. Grest. Viscosity calculations of n-alkanes by equilibrium molecular dynamics. J. Chem. Phys., 106(22):9327-9336, 1997.

[290] H. Münstedt and H. M. Laun. Elongational behaviour of a low density polyethylene melt. Rheologica Acta, 18:492-504, 1979. 
[291] Roger Edberg, Denis J. Evans, and G. P. Morriss. Constrained molecular dynamics: Simulations of liquid alkanes with a new algorithm. J. Chem. Phys., 84(12):6933-6939, 1986.

[292] Hai Hoang and Guillaume Galliero. Shear viscosity of inhomogeneous fluids. J. Chem. Phys., 136(12):124902, 2012.

[293] B. D. Todd, J. S. Hansen, and Peter J. Daivis. Nonlocal Shear Stress for Homogeneous Fluids. Phys. Rev. Lett., 100(19):195901, 2008.

[294] B. D. Todd and J. S. Hansen. Nonlocal viscous transport and the effect on fluid stress. Phys. Rev. E, 78(5):051202, 2008.

[295] J. Koplik, J. R. Banavar, and J. Willemsen. Molecular dynamics simulation of microscale Poiseuille flow and moving contact lines. Phys. Rev. Lett., 60:1282, 1988.

[296] Xi-Jun Fan, Nhan P. Thien, Ng T. Yong, and Xu Diao. Molecular dynamics simulation of a complex nano channel flow. Phys. Fluids, 14:1146, 2002.

[297] J. Koplik and J. R. Banavar. No slip condition for the mixture of two liquids. Phys. Rev. Lett., 80:5125, 1998.

[298] Filippos D. Sofos, Theodorus E. Karakasidis, and Antonios Liakopoulos. Transport properties of liquid argon in krypton nanochannels: Anisotropy and nonhomogeneity introduced by the solid walls. Int. J. Heat and Mass Transfer, 52(3-4):735743, 2009.

[299] Filippos D. Sofos, Theodoros E. Karakasidis, and Antonios Liakopoulos. Effects of wall roughness on flow in nanochannels. Phys. Rev. E, 79:026305, 2009.

[300] Filippos D. Sofos, Theodoros E. Karakasidis, and Antonios Liakopoulos. NonEquilibrium Molecular Dynamics Investigation of Parameters Affecting Planar Nanochannel Flows. Contemporary Eng. Sciences, 2:283, 2009.

[301] Filippos D. Sofos, Theodoros E. Karakasidis, and Antonios Liakopoulos. Effect of wall roughness on shear viscosity and diffusion in nanochannels. Int. J. Heat Mass Transfer, 53(1920):3839-3846, 2010.

[302] Filippos D. Sofos, Theodoros E. Karakasidis, and Antonios Liakopoulos. Surface wettability effects on flow in rough wall nanochannels. Microfluidics and Nanofluidics, 12(1):25-31, 2012. 
[303] Hossein Eslami, Farkhondeh Mozaffari, Jalil Moghadasi, and Florian MullerPlathe. Molecular dynamics simulation of confined fluids in isosurfaceisothermal-isobaric ensemble. J. Chem. Phys., 129(19):194702, 2008.

[304] Murat Barisik and Ali Beskok. Equilibrium molecular dynamics studies on nanoscale-confined fluids. Microfluidics and Nanofluidics, pages 1-14, 2011.

[305] Yun Long, Jeremy C. Palmer, Benoit Coasne, Malgorzata Sliwinska-Bartkowiak, and Keith E. Gubbins. Pressure enhancement in carbon nanopores: a major confinement effect. Phys. Chem. Chem. Phys., 13:17163-17170, 2011.

[306] Jee-Ching Wang and Kristen A. Fichthorn. A method for molecular dynamics simulation of confined fluids. J. Chem. Phys., 112(19):8252-8259, 2000.

[307] Gyoko Nagayama and Ping Cheng. Effects of interface wettability on microscale flow by molecular dynamics simulation. Int. J. Heat Mass Transfer, 47(3):501513,2004 .

[308] E. Akhmatskaya, B. D. Todd, Peter J. Daivis, Denis J. Evans, K. E. Gubbins, and L. A. Pozhar. A study of viscosity inhomogeneity in porous media. J. Chem. Phys., 106(11):4684-4695, 1997.

[309] Suresh K. Bhatia, Mauricio Rincon Bonilla, and David Nicholson. Molecular transport in nanopores: a theoretical perspective. Phys. Chem. Chem. Phys., 13:15350-15383, 2011.

[310] Hai Hoang and Guillaume Galliero. Shear behavior of a confined thin film: Influence of the molecular dynamics scheme employed. J. Chem. Phys., 138(5):054707, 2013.

[311] Hsuan-Wei Hu, George A. Carson, and Steve Granick. Relaxation Time of Confined Liquids under shear. Phys. Rev. Lett., 66:2758, 1991.

[312] J. Klein and E. Kumacheva. Simple liquid confined to molecularly thin films .I. Confinement induced liquid to solid phase transitions. J. Chem. Phys, 108:6996, 1998.

[313] E. Kumacheva and J. Klein. Simple liquid confined to molecularly thin films. II. Shear and frictional behavior of solidified films. J. Chem. Phys, 108:7010, 1998.

[314] Peter A. Thompson and Mark O. Robbins. Shear flow near solids: Epitaxial order and flow boundary conditions. Phys. Rev. A, 41:6830-6837, 1990.

[315] J. S. Hansen, B. D. Todd, and Peter J. Daivis. Prediction of fluid velocity slip at solid surfaces. Phys. Rev. E, 84:016313, 2011. 
[316] Lydéric Bocquet and Jean-Louis Barrat. Hydrodynamic boundary conditions, correlation functions, and kubo relations for confined fluids. Phys. Rev. E, 49:3079-3092, 1994.

[317] A. Jabbarzadeh, J. D. Atkinson, and R. I. Tanner. Rheological properties of thin liquid films by molecular dynamics simulations. J. Non-Newtonian Fluid Mech., 69(2-3):169-193, 1997.

[318] S. H. Krishnan and K. G. Ayappa. Glassy dynamics in a confined monatomic fluid. Phys. Rev. E, 86:011504, 2012.

[319] M. C. Stewart and R. Evans. Phase behavior and structure of a fluid confined between competing (solvophobic and solvophilic) walls. Phys. Rev. E, 86:031601, 2012.

[320] Stanislav Parez and Milan Predota. Determination of the distance-dependent viscosity of mixtures in parallel slabs using non-equilibrium molecular dynamics. Phys. Chem. Chem. Phys., 14:3640-3650, 2012.

[321] Hai Hoang and Guillaume Galliero. Local viscosity of a fluid confined in a narrow pore. Phys. Rev. E, 86:021202, 2012.

[322] Valliappa Kalyanasundaram, Douglas E. Spearot, and Ajay P. Malshe. Molecular dynamics simulation of nanoconfinement induced organization of n-decane. Langmuir, 25(13):7553-7560, 2009.

[323] D. Y. C. Chan and R. G. Horn. The drainage of thin liquid films between solid surfaces. J. Chem. Phys., 83(10):5311-5324, 1985.

[324] Jacob N. Israelachvili and Delphine Gourdon. Liquids: Putting liquids under Molecular-Scale confinement. Science, 292(5518):867-868, 2001.

[325] Karl P. Travis, B. D. Todd, and Denis J. Evans. Poiseuille flow of molecular fluids. Phys. A, 240(1-2):315-327, 1997.

[326] J. S. Hansen, Peter J. Daivis, and B. D. Todd. Local linear viscoelasticity of confined fluids. J. Chem. Phys., 126(14):144706, 2007.

[327] Junfang Zhang, B. D. Todd, and Karl P. Travis. Viscosity of confined inhomogeneous nonequilibrium fluids. J. Chem. Phys., 121:10778, 2004.

[328] Karl P. Travis, Debra J. Searles, and Denis J. Evans. On the wavevector dependent shear viscosity of a simple fluid. Mol. Phys., 97(3):415-422, 1999. 
[329] Peter J. Cadusch, B. D. Todd, Junfang Zhang, and Peter J. Daivis. A nonlocal hydrodynamic model for the shear viscosity of confined fluids: analysis of a homogeneous kernel. J. Phys. A, 41(3):035501, 2008.

[330] Chengzhen Sun, Wen-Qiang Lu, Bofeng Bai, and Jie Liu. Transport properties of ar-kr binary mixture in nanochannel poiseuille flow. Int. J. Heat Mass Transfer, 55(56):1732-1740, 2012.

[331] Hossein Eslami and Nargess Mehdipour. Local chemical potential and pressure tensor in inhomogeneous nanoconfined fluids. J. Chem. Phys., 137(14):144702, 2012.

[332] Xaioning Yang and Junqing Ding. Structure of lennard-jones fluids confined in square nanoscale channels from density functional theory. J. Chem. Phys., 121(15):7449-7456, 2004.

[333] J. M. Rickman. Elastic properties of a confined fluid. Phys. Rev. E, 86:062501, 2012.

[334] Galea T. M. and Phil Attard. Molecular dynamics study of the effect of atomic roughness on the slip length at the fluid-solid boundary during shear flow. Langmuir, 20:3477, 2004.

[335] David To. Ph.D. Thesis. The University of Adelaide, 2010.

[336] Anoosheh Niavarani and Nikolai V. Priezjev. Modeling the combined effect of surface roughness and shear rate on slip flow of simple fluids. Phys. Rev. E, 81:011606, 2010.

[337] C. Y. Soong, T. H. Yen, and P. Y. Tzeng. Molecular dynamics simulation of nanochannel flows with effects of wall lattice-fluid interactions. Phys. Rev. E, 76:036303, 2007.

[338] D. Kasiteropoulou, Theodoros E. Karakasidis, and Antonios Liakopoulos. Dissipative particle dynamics investigation of parameters affecting planar nanochannel flows. Materials Science and Eng.: B, 176(19):1574-1579, 2011.

[339] Francisco R. Hung, Benoit Coasne, Erik E. Santiso, Keith E. Gubbins, Flor R. Siperstein, and Malgorzata Sliwinska-Bartkowiak. Molecular modeling of freezing of simple fluids confined within carbon nanotubes. J. Chem. Phys., 122(14):144706, 2005.

[340] Wen-Jong Ma, Jayanth R. Banavar, and Joel Koplik. A molecular dynamics study of freezing in a confined geometry. J. Chem. Phys., 97(1):485-493, 1992. 
[341] Toshihiro Kaneko, Toshiki Mima, and Kenji Yasuoka. Phase diagram of LennardJones fluid confined in slit pores. Chem. Phys. Lett., 490(4-6):165-171, 2010.

[342] N. Gribova, A. Arnold, H. Maleki, T. Schilling, and C. Holm. 2D versus 3D Freezing of a Lennard-Jones Fluid in a Slit Pore. 2010.

[343] Vladimir P. Sokhan, David Nicholson, and Nicholas Quirke. Fluid flow in nanopores: Accurate boundary conditions for carbon nanotubes. J. Chem. Phys., 117(18):8531-8539, 2002.

[344] D. Toghraie Semiromi and A. Azimian. Nanoscale Poiseuille flow and effects of modified Lennard-Jones potential function. Heat and Mass Transfer, 46:791-801, 2010 .

[345] A. Ghosh, R. Paredes, and S. Luding. Poiseuille flow in a nanochannel-use of different thermostats. International Congress of Particle Technology, Nuremberg, Germany, pp. 1-4, CD Proceedings, 2007.

[346] Nikolai V. Priezjev. Effect of surface roughness on rate-dependent slip in simple fluids. J. Chem. Phys., 127(14):144708, 2007.

[347] F. Varnik and K. Binder. Shear viscosity of a supercooled polymer melt via nonequilibrium molecular dynamics simulations. J. Chem. Phys., 117(13):63366349, 2002.

[348] Rajesh Khare, Pawel Keblinski, and Arun Yethiraj. Molecular dynamics simulations of heat and momentum transfer at a solid-fluid interface: Relationship between thermal and velocity slip. Int. J. Heat Mass Transfer, 49(19-20):34013407, 2006.

[349] Sridhar Kumar Kannam, B. D. Todd, J. S. Hansen, and Peter J. Daivis. Slip flow in graphene nanochannels. J. Chem. Phys., 135(14):144701, 2011.

[350] J. Koplik and J. R. Banavar. Continuum Deductions from Molecular Hydrodynamics. Ann. Rev. Fluid Mech., 27(1):257-292, 1995.

[351] Jens Harting, Christian Kunert, and Jari Hyvluoma. Lattice Boltzmann simulations in microfluidics: probing the no-slip boundary condition in hydrophobic, rough, and surface nanobubble laden microchannels. Microfluidics and Nanofluidics, 8:1-10, 2010.

[352] Peter A. Thompson and Sandra M. Troian. A general boundary condition for liquid flow at solid surfaces. Nature, 389(6649):360-362, 1997. 
[353] Christian Kunert and Jens Harting. Roughness induced boundary slip in microchannel flows. Phys. Rev. Lett., 99:176001, 2007.

[354] Lyderic Bocquet and Jean-Louis Barrat. Flow boundary conditions from nanoto micro-scales. Soft Matter, 3:685-693, 2007.

[355] Alexander E. Kobryn and Andriy Kovalenko. Molecular theory of hydrodynamic boundary conditions in nanofluidics. J. Chem. Phys., 129(13):134701, 2008.

[356] S. C. Yang and L. B. Fang. Effect of surface roughness on slip flows in hydrophobic and hydrophilic microchannels by molecular dynamics simulation. Mol. Simul., 31(14-15):971-977, 2005.

[357] Jorge Castillo-Tejas, Juan F. J. Alvarado, Guillermo Gonzalez-Alatorre, Gabriel Luna-Barcenas, Isaac C. Sanchez, Ricardo Macias-Salinas, and Octavio Manero. Nonequilibrium molecular dynamics of the rheological and structural properties of linear and branched molecules. simple shear and poiseuille flows; instabilities and slip. J. Chem. Phys., 123(5):054907, 2005.

[358] William G. Hoover, Carol G. Hoover, and James F. Lutsko. Microscopic and macroscopic stress with gravitational and rotational forces. Phys. Rev. E, 79:036709, 2009.

[359] L. B. Lucy. A numerical approach to the testing of the fission hypothesis. Astron. J., 82:1013-1024, 1977.

[360] R. A. Gingold and J. J. Monaghan. Smoothed particle hydrodynamics-theory and application to non-spherical stars. Mon. Not. Roy. Astron. Soc., 181:375$389,1977$.

[361] William G. Hoover. Smooth particle applied mechanics: the state of the art. Advanced series in nonlinear dynamics. World Scientific, River Edge, 2006.

[362] Hendrik Heinz, Wolfgang Paul, and Kurt Binder. Calculation of local pressure tensors in systems with many-body interactions. Phys. Rev. E, 72(6):066704, 2005 .

[363] Kin S. Cheung and Sidney Yip. Atomic-level stress in an inhomogeneous system. J. Appl. Phys., 70(10):5688-5690, 1991.

[364] Eligiusz Wajnryb, Andrzej R. Altenberger, and John S. Dahler. Uniqueness of the microscopic stress tensor. J. Chem. Phys., 103(22):9782-9787, 1995.

[365] I. Goldhirsch and N. Sela. Origin of normal stress differences in rapid granular flows. Phys. Rev. E, 54:4458-4461, 1996. 
[366] Wikipedia. http://en.wikipedia.org/wiki/Stress_(mechanics), 2012.

[367] B. D. Todd and Denis J. Evans. The heat flux vector for highly inhomogeneous non-equilibrium fluids in very narrow pores. J. Chem. Phys., 103:9804, 1995.

[368] Kurt Binder, Jurgen Horbach, Walter Kob, Wolfgang Paul, and Fathollah Varnik. Molecular Dynamics Simulations. J. Phys. Condensed Matter, 16(5):429-453, 2004.

[369] D. C. Rapaport. The Art of Molecular Dynamics Simulation. Cambridge University Press, Cambridge, 2nd edition, 2004.

[370] B. D. Todd and Denis J. Evans. Temperature profile for Poiseuille flow. Phys. Rev. E, 55(3):2800-2807, 1997.

[371] B. D. Todd. Computer simulation of simple and complex atomistic fluids by nonequilibrium molecular dynamics techniques. Comp. Phys. Comm., 142(13):14-21, 2001.

[372] C. Goldenberg and I. Goldhirsch. Continuum mechanics for small systems and fine resolutions. Rieth, M., Schommers, W. (eds.) Handbook of Theoretical and Computational Nanotechnology, pages 330-386, 2006.

[373] Remco Hartkamp and S. Luding. Anisotropic Lennard-Jones fluids in a nanochannel. International Conference on Multiphase Flow, Tampa, Florida, pp. 1-7, CD Proceedings, 2010.

[374] H. Maleki. Ph.D. Thesis. der Johannes Gutenberg-Universität Mainz, 2011.

[375] Karl P. Travis, B. D. Todd, and Denis J. Evans. Departure from Navier-Stokes hydrodynamics in confined liquids. Phys. Rev. E, 55(4):4288-4295, 1997.

[376] J. S. Rowlinson and B. Widom. Molecular Theory of Capillarity. Dover Publications, New York, 2003.

[377] S. T. Cui, Peter T. Cummings, and H. D. Cochran. Molecular simulation of the transition from liquidlike to solidlike behavior in complex fluids confined to nanoscale gaps. J. Chem. Phys., 114(16):7189-7195, 2001.

[378] Mark E. Tuckerman, Christopher J. Mundy, Sundaram Balasubramanian, and Michael L. Klein. Modified nonequilibrium molecular dynamics for fluid flows with energy conservation. J. Chem. Phys., 106(13):5615-5621, 1997. 


\section{Acknowledgement}

I've enjoyed working on a variety of topics over the last years. While these were related to each other, they were still far enough apart so that I would have to become familiar with different theory and literature each time. This was challenging, but above all broadened my knowledge and changed the way that I approach the topic and write a scientific text. I'm grateful to many people for their help and support along the way.

In the first place, I want to thank my supervisors, Stefan and Billy. Stefan, thank you for welcoming me in your group and giving me the opportunity to work on various problems and travel to interesting places. Your thorough comments in the iterations of our papers have been extremely valuable and have taught me a lot about scientific writing. The diversity of topics in the group makes it an interesting place to be and has broadened my view tremendously. Billy, the great care and devotion that you have for your students made it really pleasant to be part of your group. You have treated me as one of your own students from the first moment that I came visiting and I immediately felt at home. Furthermore, you have introduced me to a world of interesting topics and have always been there to answer my countless questions and explain things with much patience. This has contributed to wonderful, educational and productive times in Australia.

Peter, you are not my supervisor on paper, but you deserve the same amount of credit. I may have asked as many questions to you as to Billy, and you have also taken the time to give me detailed answers and suggestions. I have always admired your thoroughness and eye for detail and I have learned a lot from working with you.

Much thanks also goes to Thomas. You have been a valuable friend and colleague over the years. I enjoyed working together and am grateful for lots of proofreading that you've done for me. Sridhar, I miss the many coffee brakes during which we discussed our work and other things during my time in Australia. Unfortunately, we were both already quite busy with finishing papers and writing our thesis, such that we couldn't work as much together as I would have liked. 
I would also like to explicitly thank Anthony, Stefano, Tom, Elnaz, Wouter and Sergio. You have all been there as a friend and colleague. We have had fun together and I've learned a lot from each of you. Furthermore, thanks to all the other collaborators, colleagues and friends who have contributed to a nice and educational four years. Also to the group secretary, Sylvia, who has always been extremely helpful and caring.

My family has always been supportive and listened patiently to me going on and on about work for the past years. Special thanks to my parents, grandparents and sister, who have always been there to listen and to advice when this was needed. The same applies to my wife. Pam, you have shown nothing but love and care during our time in Australia, Thailand, the Netherlands, and when we were far apart. Thank you for always being there for me and calming me down when I was stressed. 


\section{Curriculum vitae}

$\begin{array}{ll}\text { Name } & \text { Remco Marcel Hartkamp } \\ \text { Born } & \text { July 31, } 1985 \\ & \text { Amsterdam, The Netherlands } \\ \text { Sex } & \text { Male } \\ \text { Marital status } & \text { Married }\end{array}$

\section{Education}

- B.S. Mechanical Engineering, University of Twente, 2007.

- Minor:

Applied Mathematics, University of Twente, 2006.

- M.S. Mechanical Engineering, University of Twente, 2009.

- Thesis:

'Optimization of Airfoils in Inviscid Transonic Flow using Discrete Adjoints' prof. dr. ir. H. W. M. Hoeijmakers and dr. E. T. A. van der Weide.

- Internship:

'Particle collisions in viscous fluids', prof. M. L. Hunt., California Institute of Technology, 2008.

\section{(Long-term) visits}

- Feb-May 2008: California Institute of Technology (prof. M. L. Hunt)

- Oct-Dec 2010: Swinburne University of Technology (prof. B. D. Todd)

- Aug 2011 - Aug 2012: Swinburne University of Technology (prof. B. D. Todd) 


\section{Publications}

\section{Journal Articles}

- Remco Hartkamp, Stefano Bernardi and B. D. Todd, 'Transient-time correlation function applied to mixed shear and elongational flow', The Journal of Chemical Physics, 136, 064105 (2012)

- A. P. Markesteijn, Remco Hartkamp, S. Luding and J. Westerweel, 'A comparison of the value of viscosity for several water models using Poiseuille flow in a nano channel', The Journal of Chemical Physics, 136, 134104 (2012)

- Remco Hartkamp, A. Ghosh, T. Weinhart and S. Luding, 'A study of the anisotropy of stress in a fluid confined in a nanochannel', The Journal of Chemical Physics, 137, 044711 (2012)

- Remco Hartkamp, Peter J. Daivis and B. D. Todd, 'Density dependence of the stress relaxation function of a simple fluid', Physical Review E, 87, 032155 (2013)

- T. Weinhart, Remco Hartkamp, A. R. Thornton and S. Luding, '3D coarse grained continuum description of granular flow down an inclined surface', accepted for publication in Physics of Fluids, 2013

- Remco Hartkamp, B. D. Todd and S. Luding, 'An objective constitutive framework for the non-Newtonian pressure tensor of a simple fluid under planar flows', submitted to The Journal of Chemical Physics, 2013

- Remco Hartkamp, Sridhar Kumar Kannam, Stefano Bernardi, Debra Searles, Peter J. Daivis and B. D. Todd, 'Transient-time correlation function calculations of the shear, elongational and mixed flow rheology of linear chain molecular fluids', In preparation, 2013

- Remco Hartkamp, Thomas A. Hunt and B. D. Todd, 'A local method to calculate self-diffusion in fluids confined in a nanochannel', In preparation, 2013

\section{Proceedings}

- Remco Hartkamp and S. Luding, 'A continuum approach applied to a strongly confined Lennard-Jones fluid', Fifth International Conference on Multiscale Materials Modeling. Freiburg, Germany, October 2010

- Remco Hartkamp and S. Luding, 'Anisotropic Lennard-Jones fluids in a nanochannel', International Conference on Multiphase Flow. Tampa, Florida, May 2010 
- Remco Hartkamp, A. Ghosh and S. Luding, 'Wall-induced order in anisotropic Lennard-Jones fluid in a nanochannel', World Congress Particle Technology 6, Nuremberg, 4 pages, CD-Proceedings, April 2010 
